\title{
Electrochemical Co-Deposition of Binary Alloys
}

\author{
A Dissertation \\ Presented to \\ the faculty of the School of Engineering and Applied Science \\ University of Virginia \\ in partial fulfillment \\ of the requirements for the degree \\ Doctor of Philosophy \\ by \\ Defu Liang
}

May

2013 


\section{APPROVAL SHEET}

\section{The dissertation}

is submitted in partial fulfillment of the requirements

for the degree of

Doctor of Philosophy

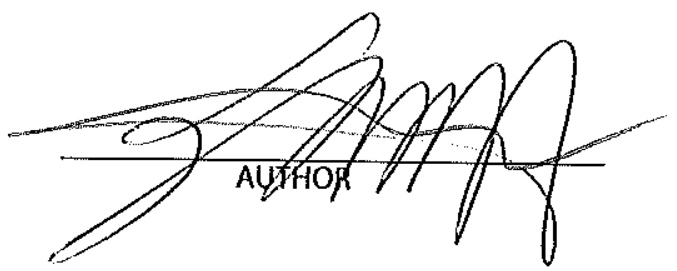

The dissertation has been read and approved by the examining committee:

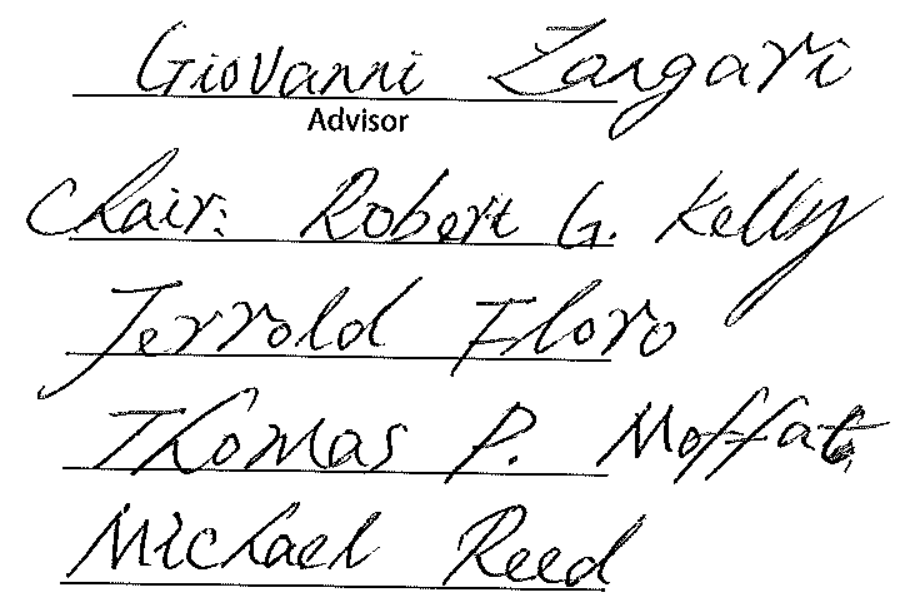

Accepted for the School of Engineering and Applied Science:

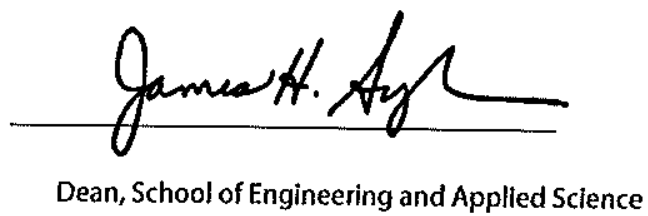

May

2013 


\title{
Electrochemical Co-Deposition of Binary Alloys
}

\author{
Defu Liang \\ Ph.D. Advisor: Dr. Giovanni Zangari \\ Materials Science and Engineering \\ University of Virginia
}




\section{Acknowledgements}

I would like to thank my advisor, Dr. Zangari, for his thorough guidance during my Ph.D. study and research in the last five years. He is a knowledgeable, intelligent and considerate person. I am deeply grateful for all your supportive and patient help all these years.

Great thanks are due to all the people who have been helping me, especially the CESE; I am really lucky to make friends with many of you, making the lab full of joy. Particular thanks go to my group mate, Dr. Jonathan J. Mallett, who has helped me through initial shock from both study and life, and continuous interaction for my improvement in research; Eric Rouya, Wenbo Shao, Lok-kun Tsui, Marcel Mibus and Michael Van Den Bossche for all the research discussion and help; project support from TE Connectivity through Zhengwei Liu and your help are also appreciated. I would like to thank Prof. William Soffa for the discussion about Fe-Pt; Priya Ghatwai and Matthew Steiner are appreciated for the experimental help and discussion. Thanks are owe to Richard White (the "Big Genius"), the facility manager for all the characterization help and letting me bother your lunch time almost every day.

I would like to thank my Ph.D. defense committee members, Dr. Robert Kelly, Jerrold Floro, Thomas Moffat and Michael Reed for their effort and dedication in ensuring my Ph.D. work.

Finally, I would like to thank my family and all my close friends; I will not be able to finish my Ph.D. without your love and support.

This dissertation is in memory of my elder brother, Delong Liang (1982-2012); the whole family is sad for your leaving; you are always with us. 


\section{Contents}

Preface

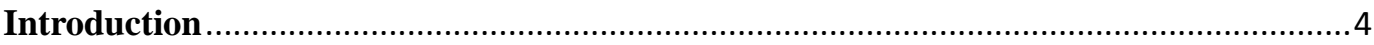

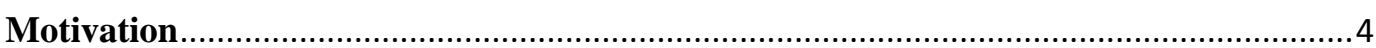

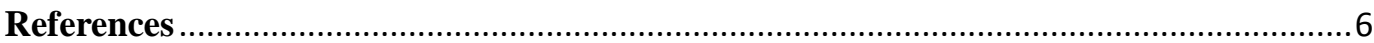

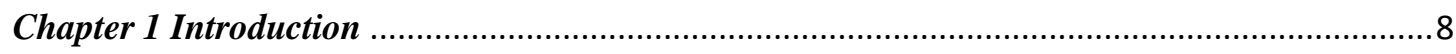

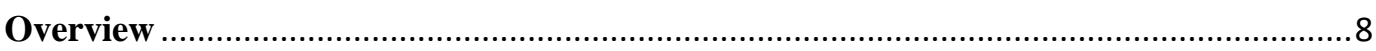

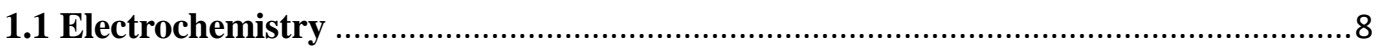

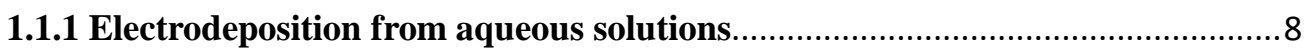

1.1.2 Faradaic and nonfaradaic processes during electrodeposition .......................11

1.1.3 Equilibrium Potential and overpotential ....................................................12

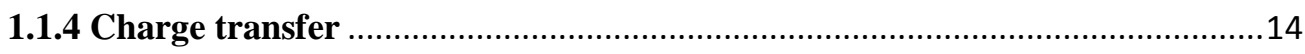

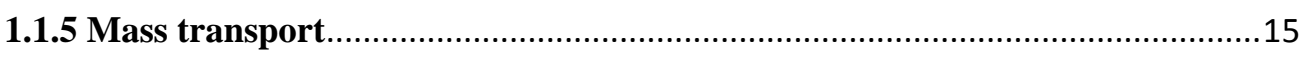

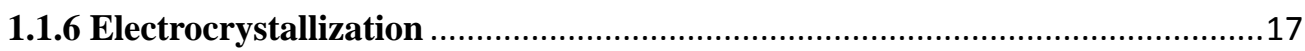

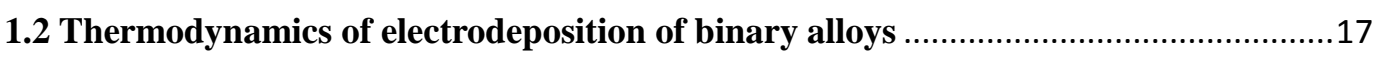

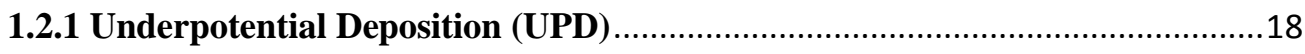

1.2.2 Thermodynamics of binary alloys........................................................... 18

1.2.3 Electrodeposition of solid solution type binary alloys....................................20

1.2.4 Electrodeposition of binary alloys with miscibility gap ...............................22

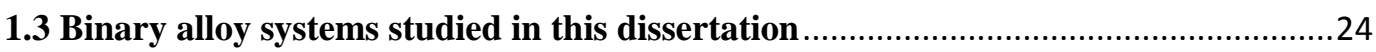

1.3.1 Cu-Au with bulk negative heat of mixing and no side hydrogen evolution

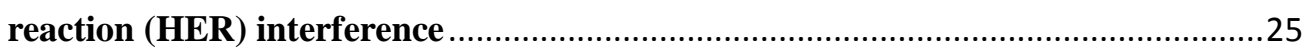

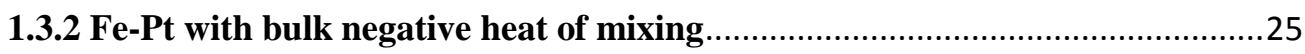

1.3.3 Au-Ni with bulk positive heat of mixing ....................................................26

1.3.4 Ag-Cu with bulk positive heat of mixing and a eutectic alloy system .............26

1.3.5 Ag-Ni with bulk positive heat of mixing ...................................................27

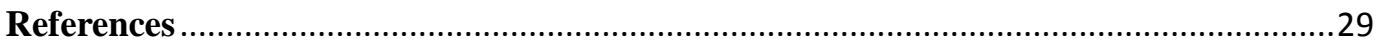

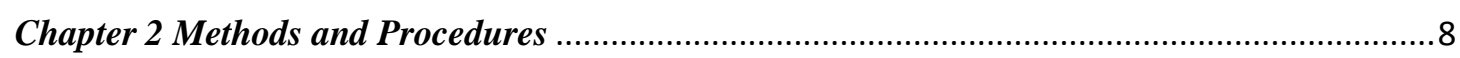

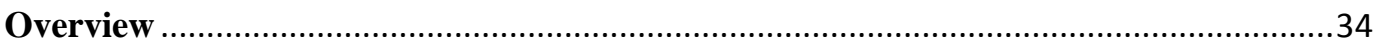

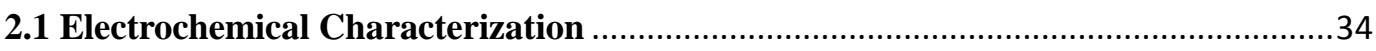

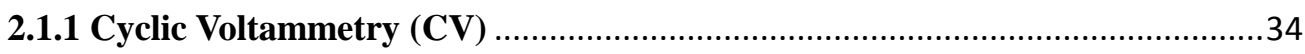

2.1.2 Electrochemical Quartz Crystal Microbalance (EQCM) ................................34

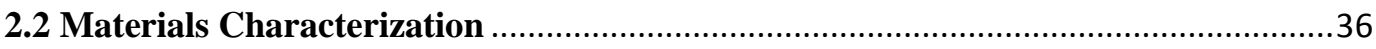

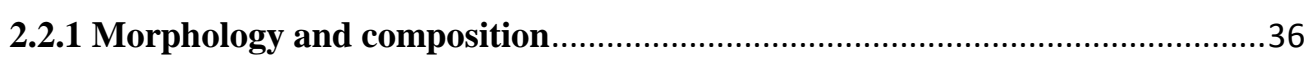

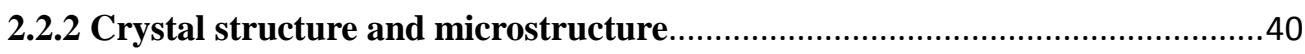

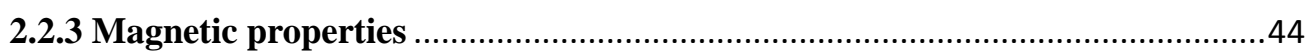

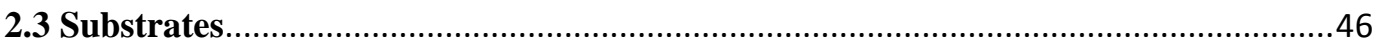

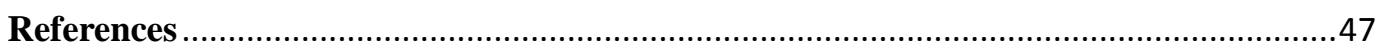

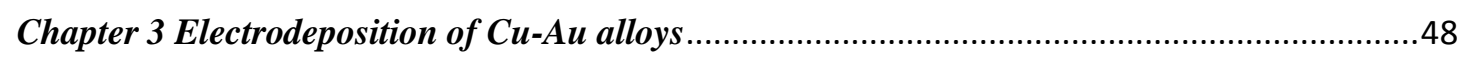

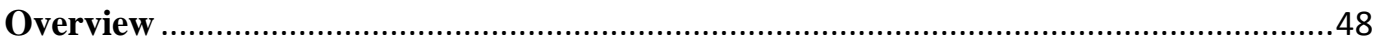

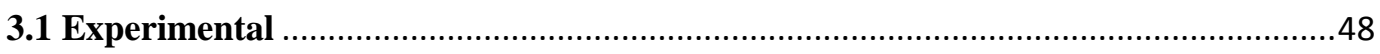

3.2 Electrodeposition of Au-Cu alloys from weakly complexed solutions ........................50 


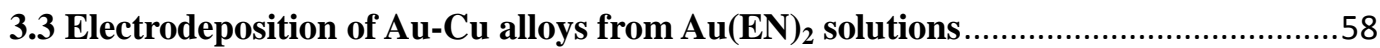

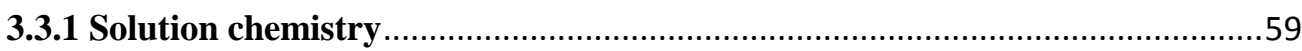

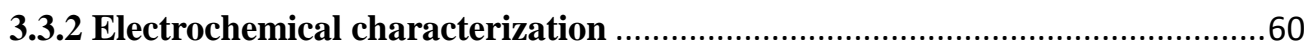

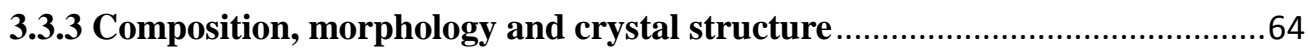

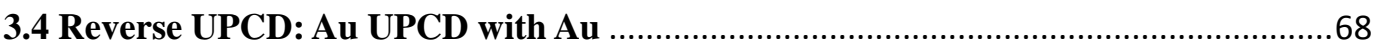

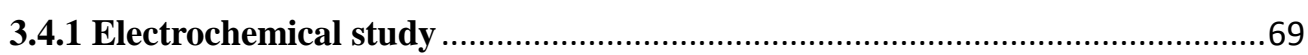

3.4.2 Composition, morphology and crystal structure ......................................... 71

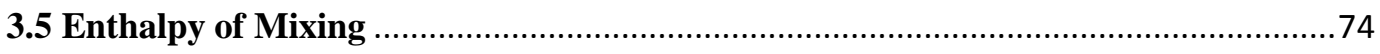

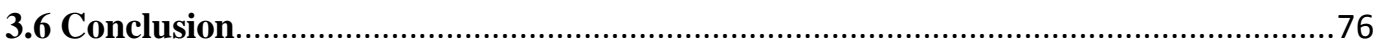

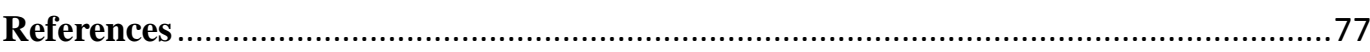

Chapter 4 Electrodeposition of Fe-Pt alloys from an alkaline solution .......................................79

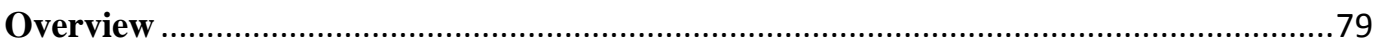

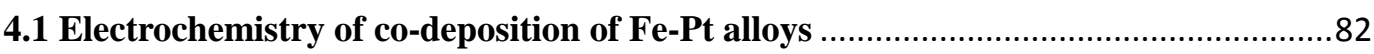

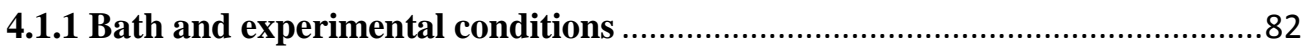

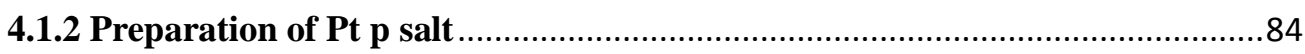

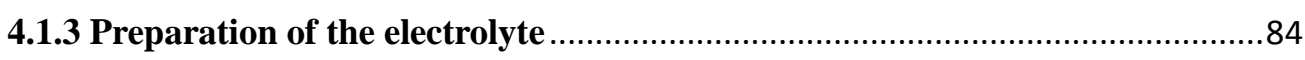

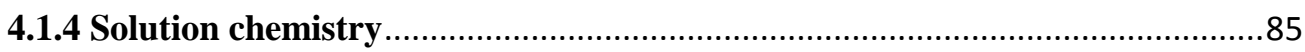

4.1.5 Electrochemical study on Fe-Pt from the alkaline solution .............................89

4.2 Morphology, crystalline structure and magnetic properties of single layer Fe-Pt

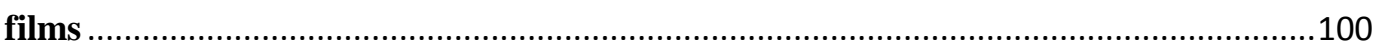

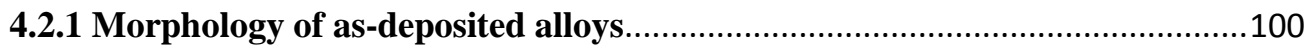

4.2.2 Crystalline structure and magnetic properties ..........................................102

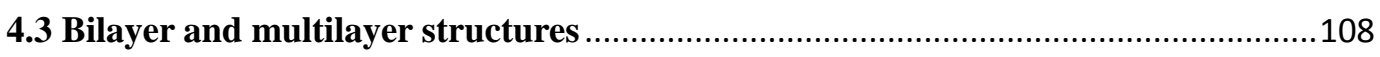

4.3.1 Electrodeposition of compositionally graded multilayers.............................109

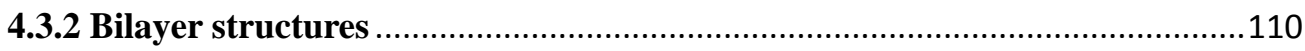

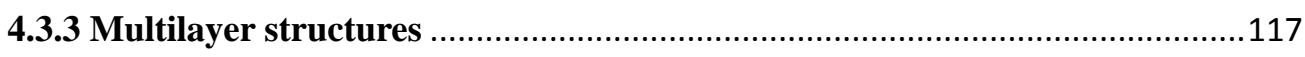

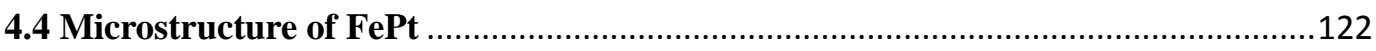

4.5 Crystal structure, microstructure of $\mathrm{Fe}$ rich and $\mathrm{Fe}_{3} \mathrm{Pt}$ alloys ..............................124

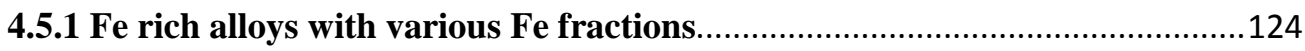

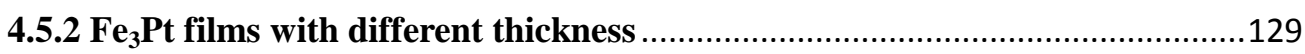

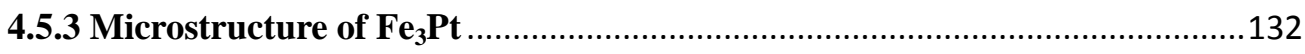

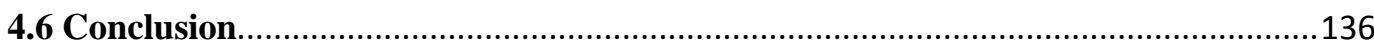

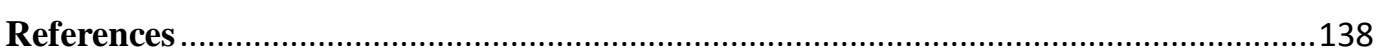

Chapter 5 Electrodeposition of immiscible Au-Ni alloys ........................................................ 141

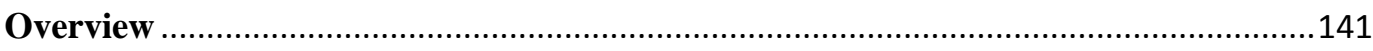

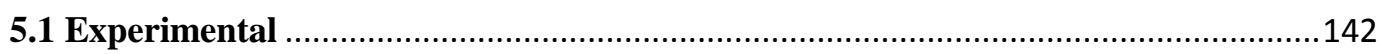

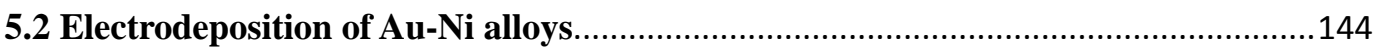

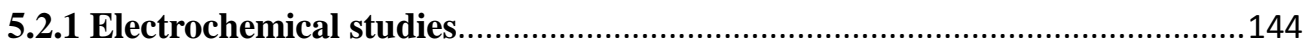

5.2.2 Composition, morphology and crystal structure of electrodeposited Au-Ni

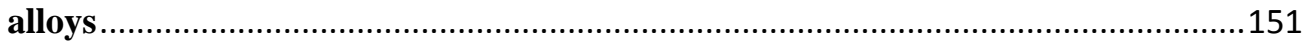

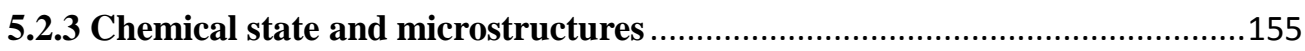

5.3 Thermodynamics of electrodeposition of Au-Ni alloys ...........................................159

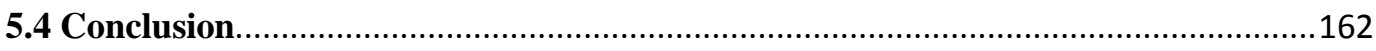

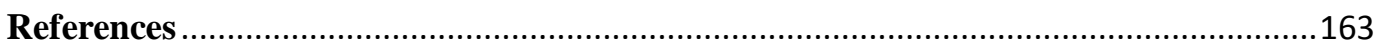




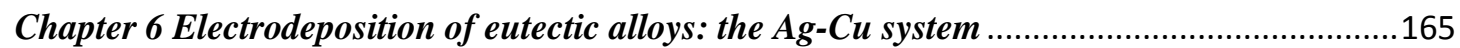

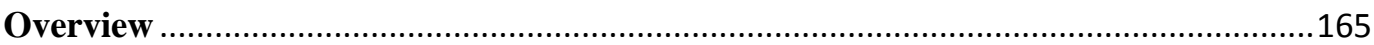

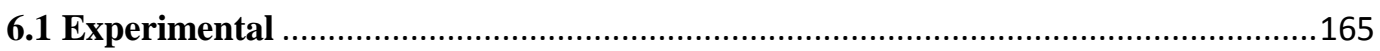

6.2 Electrodeposition of Ag-Cu from non complexing acidic solutions ...........................167

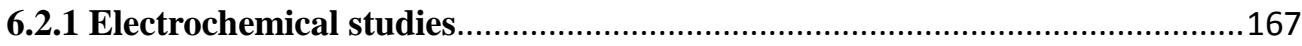

6.2.2 Composition, morphology and crystal structure of Ag-Cu deposits..............171

6.2.3 Resistivity of Ag-Cu alloys .................................................................176

6.3 Electrodeposition of Ag-Cu from TU complexing solutions ………………...........177

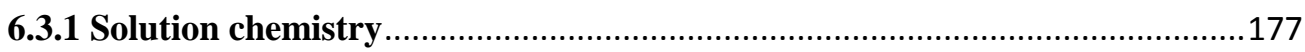

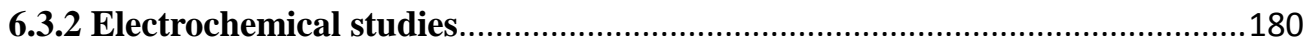

6.3.4 Composition, Morphology and Crystal structure of Ag-Cu deposits .............186

6.3.5 Resistivity of Ag-Cu alloys from complexing solution.....................................191

6.4 Crystal structure of $\mathrm{Ag}-\mathrm{Cu}$ alloys grown from non complexing and complexing

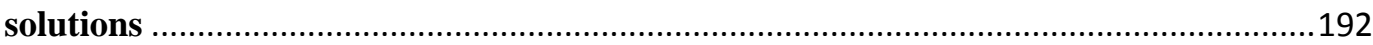

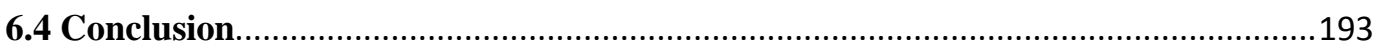

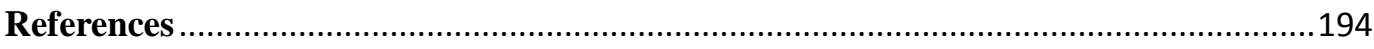

Chapter 7 Electrodeposition of Ag-Ni alloys from thiourea complexing solutions ....................196

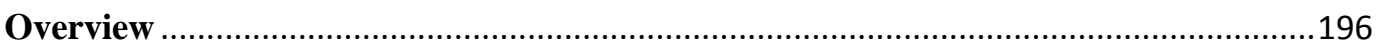

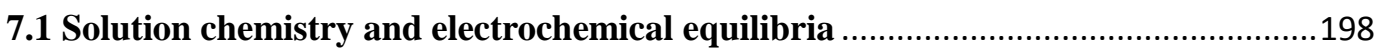

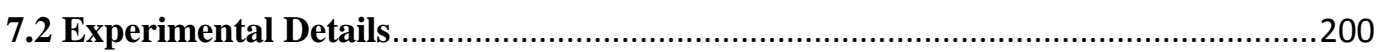

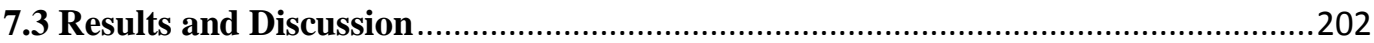

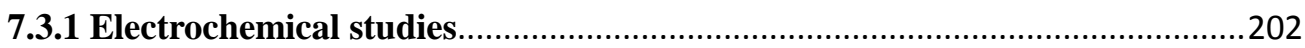

7.3.2 Compositions, Morphology, crystal structure and microstructures ..............204

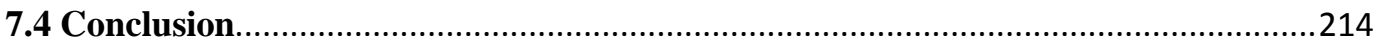

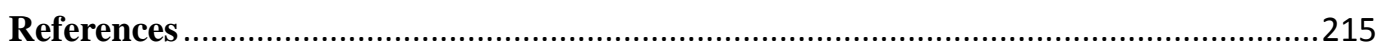

Chapter 8 Conclusion and Recommendation for Future Work …….......................................218

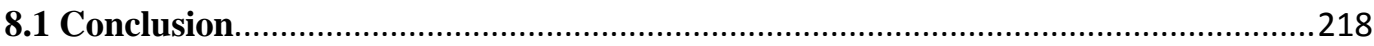

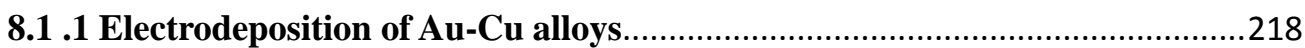

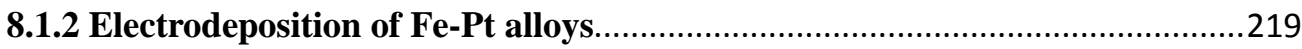

8.1.3 Electrodeposition of Au-Ni alloys..........................................................220

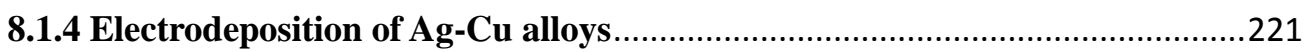

8.1.5 Electrodeposition of Ag-Ni alloys ............................................................222

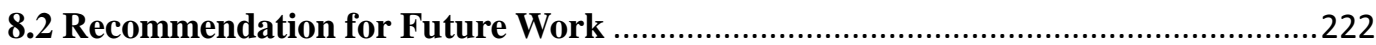




\section{Preface}

\section{Introduction}

Devices fabricated with single metallic and alloy thin films on suitable substrates, such as electronics, magnetic microelectromechanical systems (MEMS), magnetic sensor and energy conversion devices [1,2], are widely utilized nowadays,. With the requirement of miniaturization, the control of deposited thin films with precise structures, phases and compositions for alloy films, therefore the desired properties is highly challenging.

Various deposition techniques have found their application in thin film deposition, e.g., sputtering [3], laser deposition [4], and molecular beam epitaxy [5]. Electrochemical deposition (ECD) possesses advantages of low cost, better control of driving force for growth and non vacuum requirement traditionally; this has been well known for its applications in industrial levels, such as metal coatings. With the introduction of $\mathrm{Cu}$ interconnects in ultra large scale systems [6-8], ECD has been shown to be reliable in advanced microelectronics fabrications. Particularly, it is unique in filling very small features, capable of avoiding the line of sight flaws in most vacuum techniques, rendering it promising for nanostructure designs, pattern magnetic recording for example [9].

\section{Motivation}

Studies on the deposition mechanism, especially focusing on the nucleation and growth process of electrodeposition of single metals has been intensively carried out, such as $\mathrm{Cu}[10-12]$ and $\mathrm{Ni}[13,14]$. Comparing with their corresponding single components, alloys of metals possess tunable properties [15-17]. For example, electrodeposited hard gold alloys by alloying usually less than 1at.\% of Co with $\mathrm{Au}$ help to greatly increase their hardness, while the electrical properties, wear resistance 
and resistance to oxidation remain similar [18,19]; equiatomic FePt alloy with an ordered structure of $\mathrm{L}_{0}$ phase possesses coercivity of tens of $\mathrm{kOe}$, while Fe itself is magnetically soft and Pt nonmagnetic [20]. From the synthesis point of view, it will be highly desirable to construct the relation between external parameters of electrodeposition, e.g., applied potential or current density, to the obtained compositions, phases and structures and therefore properties.

In this dissertation, we aim to study the effect of solid state thermodynamics (mainly the enthalpy of alloy formation) on the compositions, phases and structures of the alloys. Under relatively slow deposition rates, with the assumption of close to thermodynamic equilibrium, we evaluate the ability to predict binary composition and structures of binary alloys using the enthalpy of formation through the underpotential co-deposition (UPCD) of active elements with noble elements. Five alloy systems with different alloying behaviors and of fundamental and technological importance will be assessed: Au-Cu with negative enthalpy and both elements can be deposited without parasitic hydrogen evolution reaction (HER); Fe-Pt with negative enthalpy and excellent hard magnetic properties near equiatomic compositions; Au-Ni with positive enthalpy but $\mathrm{Au}-\mathrm{Ni}$ pairs tend to form preferentially with respect to homologous ones; Ag-Cu with positive enthalpy and unlike pairs not favored; Ag-Ni with positive enthalpy and a miscibility gap even in liquid state. 


\section{References}

[1] M. Datta, Electrochimi. Acta 45 (2000) 2535.

[2] L.P. Bicelli, B. Bozzini, C. Mele, L.D. Urzo, Int. J. Electrochem. Sci. 3 (2008) 356.

[3] L.B. Barthélémy, A., Cros, V., Duvail, J.L., Fert, A., Morel, R., Parent, F., Petroff, F., Steren, Nanostruct. Mater. 6 (1995) 217.

[4] R. van Ingen, R. Fastenau, E. Mittemeijer, Phys. Rev. Lett. 72 (1994) 3116.

[5] C. Azizi, A., Thompson, S.M., Ounadjela, K., Gregg, J., Vennegues, P., Dinia, A., Arabski, J., Fermon, J. Magn. Magn. Mater. 148 (1995) 313.

[6] P.C. Andricacos, C. Uzoh, J.O. Dukovic, J. Horkans, H. Deligianni, IBM J. Res. Dev. 42 (1998) 567.

[7] T.P. Moffat, D. Wheeler, M.D. Edelstein, D. Josell, IBM J. Res. Dev. 49 (2005) 19.

[8] T.P. Moffat, J.E. Bonevich, W.H. Huber, A. Stanishevsky, D.R. Kelly, G.R. Stafford, D. Josell, J. Electrochem. Soc. 147 (2000) 4524.

[9] C. Ross, Annu. Rev. Mater. Sci. 31 (2001) 203.

[10] I.R. Burrows, J.A. Harrison, J. Thompson, J. Electroanal. Chem. Interfacial Electrochem. 58 (1975) 241.

[11] D. Grujicic, Electrochim. Acta 47 (2002) 2901.

[12] W. Shao, G. Pattanaik, G. Zangari, J. Electrochem. Soc. 154 (2007) D201.

[13] A. Saraby-Reintjes, Electrochim. Acta 29 (1984) 557.

[14] K.S. Kumar, S. Suresh, M.F. Chisholm, J. a. Horton, P. Wang, Acta Mater. 51 (2003) 387.

[15] A.M. Molenbroek, J.K. Nørskov, B.S. Clausen, J. Phys. Chem. B 105 (2001) 5450-5458.

[16] J.J. Mallett, E.B. Svedberg, S. Sayan, a. J. Shapiro, L. Wielunski, T.E. Madey, W.F. Egelhoff, T.P. Moffat, Electrochem. Solid-State Lett. 7 (2004) C121.

[17] R. Winand, Electrochimi. Acta 39 (1994) 1091. 
[18] C.C. Lo, J. Appl. Phys. 50 (1979) 6887.

[19] W. Chrzanowski, Y.G. Li, A. Lasia, J. Appl. Electrochem. 26 (1996) 385.

[20] O. Gutfleisch, J. Lyubina, K.-H. MÜller, L. Schultz, Adv. Eng. Mater. 7 (2005) 208. 


\section{Chapter 1 Introduction}

\section{Overview}

Since this dissertation focuses on the electrodeposition of binary alloys, seeking connections with the solid state thermodynamics, it is necessary to briefly overview some basic concepts of both electrochemistry and alloying thermodynamics. Thus, this chapter will cover three main topics: electrochemical fundamentals of electrodeposition from aqueous solutions; thermodynamics in electrodeposition of binary alloys; selected binary alloy systems for this thesis.

\subsection{Electrochemistry}

\subsubsection{Electrodeposition from aqueous solutions}

Generally, electrodeposition has been conducted in aqueous solutions. Nowadays, electrodeposition has been extended to media consisting of organic solvents [1,2], molten salts [3,4] or ionic liquids [5]. However, deposition from aqueous solutions is the only technique used at an industrial level and cost efficient as well. We will only focus on electrochemistry of deposition from aqueous solutions as a result.

Electrodeposition is the process during which metal ions are reduced and incorporated into the solid metal form through the electrode-electrolyte interface in an electrochemical cell. A typical cell consists of an anode or counter electrode, a cathode or working electrode and a reference electrode. The reaction can be simplified as in Equation (1.1) without complexing or (1.2) with complexing agent, where $M e^{z+}$ is the metal ion in the electrolyte, and $L$ the complexing agent.

$$
\begin{gathered}
M e^{z+}+z e^{-} \Leftrightarrow M e \\
{\left[M e_{x} L_{y}\right]^{z+}+z e^{-} \Leftrightarrow x M e+y L}
\end{gathered}
$$


The complicated process occurs within the electrode-electrolyte interface, and thus it is the heart of electrodeposition. Consider the simple case of immersing a metal Me into an electrolyte containing its salt MeA. Upon immersing, some ions from the solution are reduced and incorporated into the metal lattice, while other metal atoms are oxidized and enter the solution due to the difference of electrochemical potentials of the two. A dynamic equilibrium of ion exchange will be established once thermodynamic equilibrium is reached. This will lead to an excess of electrons on the metal side $q_{M e}$ (charges per metal area). Correspondingly, charges on the solution side will rearrange in response to the metal side and positive metal ions will accumulate on the solution of the interface. At equilibrium, the interface region is charge neutral:

$$
q_{M e}=-q_{S}
$$

where $q_{S}$ is the charge per unit area on the solution side of the interface.

Models have been proposed to study the properties of the interface since more than a century: the Helmholtz model, Gouy-Chapman model, and Stern model and Grahame model. Helmholtz double layer model dates back to 1874 [6]. It assumes that all excess charge on the metal side is located on the metal surface, while the excess charge on the solution side is lined up on a same plane parallel at a certain distance, $d$, from the metal surface, which is termed the Helmholtz plane. The distance $d$ is determined by the hydration sphere of the metal ion. The interface made of two parallel planes with opposite charge is termed the double layer, and is equivalent to a parallel plate capacitor, for which the capacity can be calculated as following,

$$
C=\frac{\varepsilon}{4 \pi d} \quad\left[\mathrm{~F} / \mathrm{cm}^{2}\right]
$$

where $\varepsilon$ is the dielectric constant of the medium between the two plate and $d$ the distance between the charged layers.

As can be seen from equation (1.4), the capacitance of the double layer is independent of electrode potential in the Helmholtz model, which contradicts experimental observations. A new model was proposed by Gouy and Chapman independently 
assuming ions can be modeled as a point charge and they follow the Boltzmann distribution. This model predicts a potential-dependent capacitance of the double layers, and is obeyed very well in dilute solutions and at potential near the minimum capacitance; it fails to predict the capacitance for concentrated solutions and electrode potentials far away the minimum capacitance, however.

The later Stern model combines the Helmholtz model and the Gouy-chapman model by separating the charge in the solution side into two parts: some excess of the charge $q_{H}$ is restricted at a fixed plane, the Helmholtz plane, close to the metal surface, while other charge $q_{G C}$ is statistically distributed into the solution. The interface thus is composed of two regions: the compact region and the diffuse layer:

$$
\begin{gathered}
q_{s}=q_{H}+q_{G} \\
\frac{1}{C}=\frac{1}{C_{H}}+\frac{1}{C_{G C}}
\end{gathered}
$$

The Stern model predicts very well trends for the same solution with different concentrations, but fails to distinguish the behaviors of different solutions.

The more recent Grahame model introduces an inner plane of closest approach, or inner Helmholtz plane (IHP). IHP is the center of partially or fully dehydrated ions; the closest location of fully hydrated ions is at another plane farther from the metal electrode, the outer Helmholtz plane (OHP). Thus, the Grahame model is also called triple-layer model and is shown in Fig. 1.1 [7]. It shows better match with the experimental observations. 


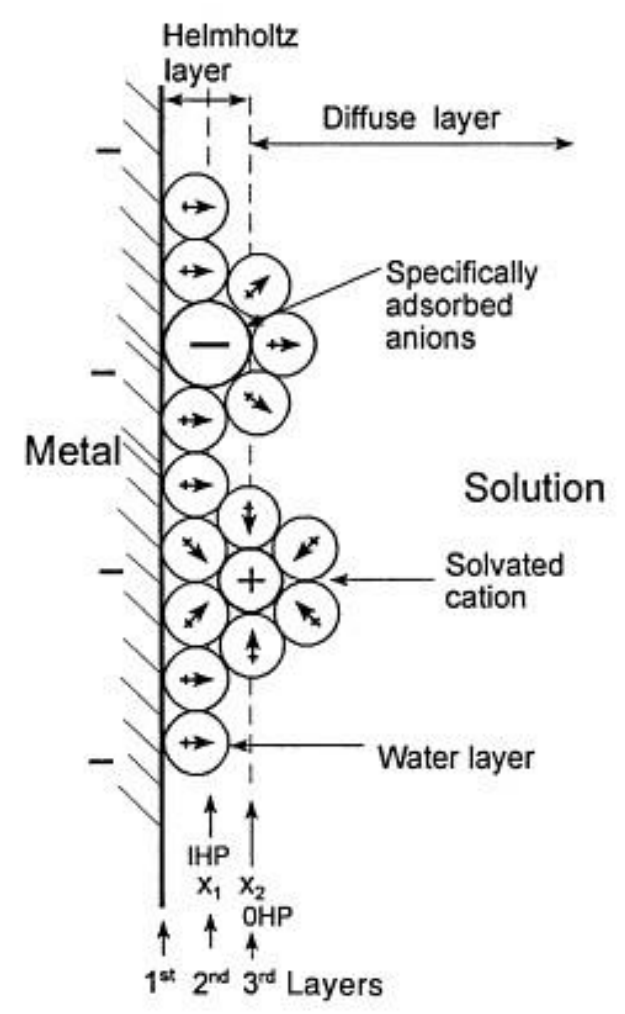

Figure 1.1 The triple-layer model [7].

\subsubsection{Faradaic and nonfaradaic processes during electrodeposition}

The double layer is formed under equilibrium. Upon applying a potential to the electrode, two types of processes can occur: 1) electrons are transferred across the electrode-electrolyte interface, leading to reduction or oxidation; such reactions are governed by the Faraday's Law and termed Faradaic processes; 2) within some potential range, charge-transfer reactions are not thermodynamically or kinetically favored and thus cannot occur. However, processes such as ion or molecule adsorption or desorption can occur; double layer structure can change dependent on applied potentials. These processes are called nonfaradaic processes. We will focus only on faradaic processes in the following. 


\subsubsection{Equilibrium Potential and overpotential}

Faradaic processes that involve charge-transfer reactions are crucial in electrodeposition. Under equilibrium, there is no net current flowing through the interface; this potential is termed Equilibrium potential $E_{e q}$. However, this is a dynamic equilibrium: that is, the partial current $\vec{i}$ corresponding to the cathodic reduction is equal to the partial current $\overleftarrow{i}$ for the anodic oxidation current, leading to the zero net current. The corresponding partial current density is defined as exchange current density of the system $j_{0}$. It is an intrinsic property of the system and is determined by the electrochemical reaction, the electrolyte, the electrodes and temperatures. It is therefore one of the most important parameters in electrochemical kinetics. When a potential $E(i)$ deviating from the equilibrium potential is applied on the electrodes, current i flows through the elelctrodes, and is dependent on the applied potential. The equilibrium potential $E_{e q}$ can be calculated by the Nernst equation:

$$
E_{e q}=E_{0}+\frac{R T}{z F} \ln \frac{\left[M e^{z+}\right]}{[M e]}
$$

where $E_{0}$ is the standard electrode potential, defined as the equilibrium potential for the system with unity activity for all species involved, $R$ the gas constant, $F$ the Faraday constant. The activity of electrons and metal atoms are conventionally defined as unity.

Conventionally, the difference between applied potential and the equilibrium potential is termed overpotential:

$$
\eta=E(i)-E_{e q}
$$

During the overall process of electrodeposition, several successive atomistic steps 
occur (Fig.1.2):

1) The transport process: the species to be reduced diffuse from the bulk electrolyte to the cathode/ electrolyte interface;

2) The charge-transfer and adsorption process: the species exchange electrons with the conductive solid cathode (termed as working electrode) and lose part or all charge, becoming adatoms or adions on the electrode surface;

3) Surface diffusion and nucleation: the adatoms or adions diffuse to and are incorporated in the kink sites; 2-D or 3-D clusters are formed;

4) Growth: the propagation of the steps or the growth of clusters with the formation of stable nuclei and the formation of the bulk deposited phases.

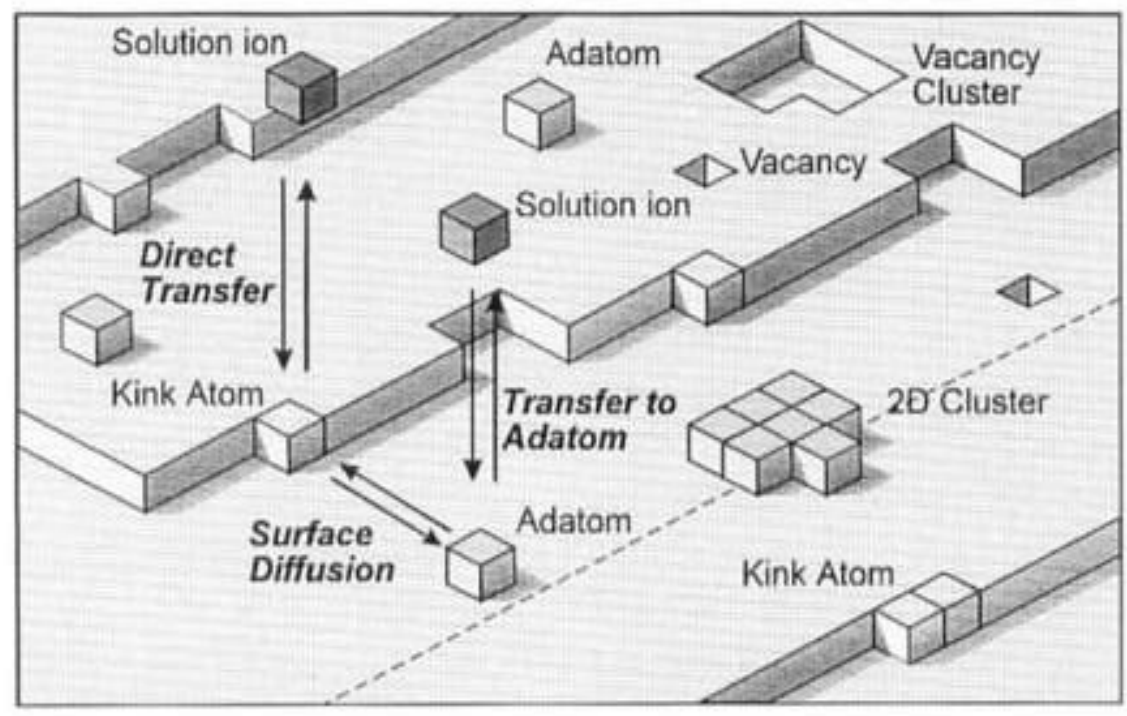

Figure 1.2 Schematic diagram showing the steps during electrodeposition [8].

There are energy barriers related with all of these steps, and charge-transfer, species surface diffusion and crystallization are directly dependent on the overpotential. Therefore, the overall overpotential can be written in the following format:

$$
\eta=\eta_{c t}+\eta_{d}+\eta_{c}
$$

where $\eta_{c t}, \eta_{d}, \eta_{c}$ are the charge-transfer, diffusion and crystallization overpotetnials, respectively. Similar to chemical reactions, the overall deposition rate is determined by the slowest step. 
We will address these three parts in more detailed in the following sections.

\subsubsection{Charge transfer}

As discussed above, when a potential different from the equilibrium value is applied on the electrode, the net current is no longer zero; it is dependent on the applied potential. The net current density $j$ for a $\mathrm{z}$ electron transfer reaction is often given by the Bulter-Volmer equation:

$$
j=\vec{j}-\overleftarrow{j}=j_{0}\left[\exp \left(\frac{-z \alpha F \eta}{R T}\right)-\exp \left(\frac{(1-\alpha) z F \eta}{R T}\right)\right]
$$

where $\alpha$ is the charge transfer coefficient which is related to the shape of the energy barrier, $F$ the Faraday constant, $R$ the gas constant and $T$ the absolute temperature.

Depending on the magnitude of the overpotential, the mathematical expression can be simplified. When the overpotential is small, i.e., the applied potential is close to the equilibrium potential, the linear approximation can be applied:

$$
j=j_{0}\left[\left(\frac{-z \alpha F \eta}{R T}\right)-\left(\frac{(1-\alpha) z F \eta}{R T}\right)\right]=j_{0} \frac{z F}{R T} \eta
$$

In this case, the net current density varies linearly with the overpotential; this usually works well when the overpotential is less than $10 \mathrm{mV}$.

When the overpotential is large, and negative (this is usually the case for cathodic deposition), the cathodic current increases exponentially with overpotential, while the anodic current decreases exponentially with $\eta$ and can be neglected. The net current density can be rearranged as:

$$
j=\vec{j}=j_{0}\left[\exp \left(\frac{-z \alpha F \eta}{R T}\right)\right]
$$

This equation can be further written as the Tafel equation:

$$
\eta=a-b \log j
$$

where $\mathrm{a}$ and $\mathrm{b}$ are constants for a given reaction and a given temperature defined as follows. 


$$
\begin{gathered}
a=\frac{2.303 R T}{\alpha z F} \log j_{0} \\
b=\frac{2.303 R T}{\alpha z F}
\end{gathered}
$$

The Tafel equation is usually used to fit expetrimental data of $j-\eta$ plot. The slope $b$ is termed the Tafel slope. It provides very useful information about the mechanism of multistep reactions. This relation holds only within a limited potential range, called the kinetic-controlled region.

\subsubsection{Mass transport}

When the cathodic overpotential is very large, the overall deposition rate is determined by the transport of species to the interface. For the solutions used in electrodeposition, supporting electrolytes are usually used to increase the conductivity of the solution and reduce the ohmic potential drop. As a result, the migration for the ion under study is very small due to the fact that the larger portion of migration is attributed to the non-reactive supporting electrolyte ions, and diffusion is the only mass transport mechanism in the system. Initially, the electrolyte has a homogenous concentration of ionic species. When a cathodic potential is applied to the electrode, species near the electrode are reduced and the region is soon depleted of ions. A simplified model, the Nernst diffusion model is used to estimate the ion distribution. This assumes a linear drop of the concentration of the metal ions from bulk $\left[\mathrm{Me}^{\mathrm{z+}}\right]$ at a distance $\delta$ to the electrode surface, to zero on the electrode surface; $\delta$ is the thickness of the diffusion layer, which is a function of time:

$$
\delta=2 \sqrt{D_{M e}} \sqrt{\frac{t}{\pi}}
$$

where $D_{M e}$ is the diffusivity of metal ion in the solution. As can be seen, $\delta$ increases with the square root of time. In the usual experimental setup for stagnant electrolytes, due to the natural convection, the thickness of the diffusion layer is $\sim 10-20 \mu \mathrm{m}$, depending on the electrolyte viscosity and the cell geometry; beyond that, 
the concentration of metal ions is the same as the bulk.

Under mass transport control, as soon as the metal ions diffuse to the electrode surface they are reduced; the overall deposition rate is determined by the diffusion current:

$$
j_{L}=\frac{z F D_{M e} C_{b}}{\delta}
$$

A general current-potential relationship usually includes both charge-transfer and mass transport controlled regions. At relatively low overpotential, it follows the Bulter-Volmer behavior; at very large overpotential, current density reaches the diffusion limited current and is constant. A typical plot for these behaviors is shown in Fig. 1.3.

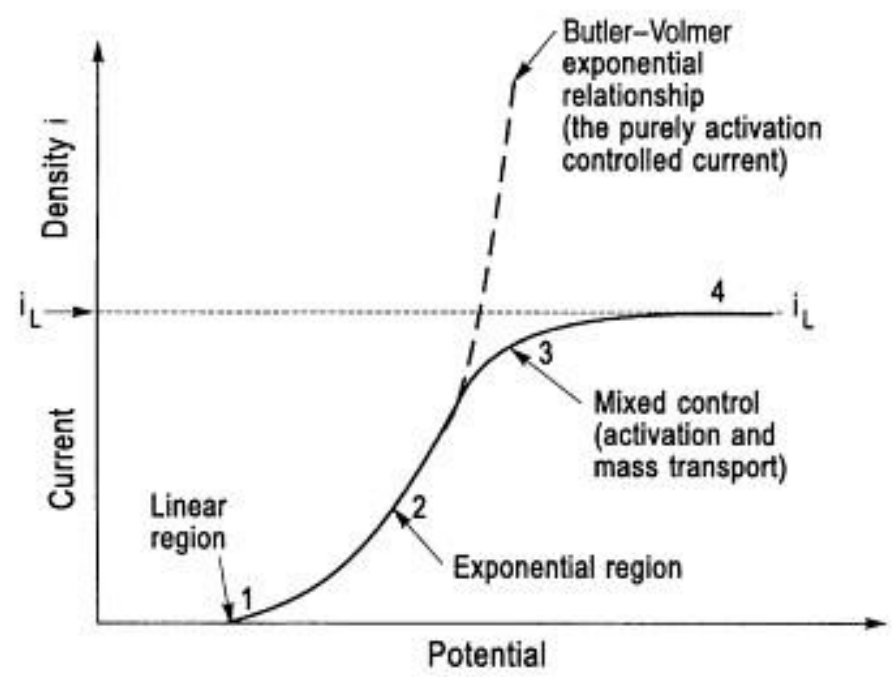

Figure 1.3 Four regions in the general current-overpotential relationship [7].

Mass transport control of electrodeposition is of practical importance. It usually leads to the formation of rough or porous structures, dendrites for example [9]. 


\subsubsection{Electrocrystallization}

The substrate (the working electrode) surface usually exhibits various kinds of defect, as shown in Fig.1.2. These include steps, terraces, kink sites or vacancies. These defects have a higher number of bonds available for the reduced adatoms and are energetically favorable for nucleation, if necessary, or for growth sites. The nucleation process usually involves two stages: 1) charge-transfer: metal ions diffuse to the electrode and they are reduced, forming adatoms on the electrode surface; 2) the adatoms diffuse by random walk and collide with other adatoms, forming a nucleus. This nucleus may not be stable and it can fall apart; this is governed by thermodynamics. The increase of the Gibbs free energy of a nucleus with $\mathrm{N}$ adatoms is written as:

$$
\Delta G=-N z e|\eta|+\phi(N)
$$

where the first term is the free energy change for the transformation of $\mathrm{N}$ ion to adatoms in the nucleus; the second term the increase of surface energy due to the formation of the nucleus and is always positive. The criterion for the stability of the nucleus is to minimize the energy change on the formation of the nucleus. Take a 2-D cluster as an example, the critical nucleus radius is given by equation (1.19) considering minimizing free energy in equation (1.18):

$$
r_{c}=\frac{s \varepsilon}{z e \eta}
$$

where $s$ is the area occupied by one adatom on the nucleus surface and $\varepsilon$ the edge energy. As clearly shown, the critical radius is inversely proportional to the overpotential. In other words, at higher overpotentials, smaller nuclei can exist.

\subsection{Thermodynamics of electrodeposition of binary alloys}

Electrodeposition of alloys can extend the fabrication and application opportunities comparing with corresponding pure metal constituents, providing better and more tunable properties. However, the deposition process of alloys is more complicated, 
especially to achieve the uniformity in thickness and composition. Up till now, there is no general theory for electrodeposition of alloys, but only studies for some specific systems due to their practical importance of applications.

\subsubsection{Underpotential Deposition (UPD)}

Before discussing about alloy co-deposition, it is necessary to introduce a related phenomenon, underpotential deposition (UPD). UPD is a phenomenon that a reactive metal (M) can be electrodeposited on a more noble substrate (S) at potentials more positive than depositing onto itself $[10,11]$. The positive shift in deposition potential is due to the attractive interaction between $\mathrm{S}$ and $\mathrm{M}\left(\mathrm{V}_{\mathrm{SM}}\right)$ being stronger than that between $\mathrm{M}$ and $\mathrm{M}\left(\mathrm{V}_{\mathrm{MM}}\right)$; the effective pair interaction $\mathrm{V}_{\text {eff }}=\mathrm{V}_{\mathrm{SS}}+\mathrm{V}_{\mathrm{MM}}-2 \mathrm{~V}_{\mathrm{SM}}$ is of the order of 0.01-0.1 eV[12]. As a result, only a monolayer or two is deposited on the substrates underpotentially. By manipulating UPD conditions, it is shown that film thickness can be well controlled [13].

\subsubsection{Thermodynamics of binary alloys}

When two constituents are brought and mixed together, no matter by what kind of methods, the interaction between them usually exists. Thermodynamically, the mixing behavior can be described by the Gibbs free energy change between before and after mixing:

$$
\Delta G_{m i x}=\Delta H_{m i x}-T \Delta S_{m i x}
$$

For electrodeposition from aqueous solutions, experiments are performed at low temperatures $\left(<100^{\circ} \mathrm{C}\right)$, usually at room temperature; thus the change of entropy term can be neglected, and the free energy of mixing can be approximated by the enthalpy of mixing. If the sub-regular solution [14] is used, then the enthalpy of mixing can be written as:

$$
\Delta H_{m i x}=x_{1}\left(1-x_{1}\right)\left[W_{1}\left(1-x_{1}\right)+W_{2} x_{1}\right]
$$

where $\mathrm{x}_{1}$ is the atomic fraction of one element, and $W_{1}$ and $W_{2}$ two composition and 
temperature independent parameters.

For systems with the formation of solid solutions favored, enthalpy of mixing is negative; for eutectic alloys, they are characterized by positive enthalpy.

For electrodeposition of binary alloys, the Nernst' equation in (1.7) can be rewritten as:

$$
\operatorname{Eeq}(i)=E_{0}+\frac{R T}{z F} \ln \frac{a_{i}^{\text {sol }}}{a_{i}^{\text {alloy }}}
$$

where $a_{i}^{\text {sol }}$ is the activity of constituent $\mathrm{i}$ in solution, which usually can be approximated by its free ion concentration, and $a_{i}^{\text {alloy }}$ the activity of $\mathrm{i}$ in the deposited alloys. In most situations, the equilibrium potentials for the two metals are usually different, and for convenience, here we define the one with more positive potential as noble metal, and the other one reactive metal. Since when only the noble metal is deposited, there is no solid interaction, we will only have to consider the situation when the reactive metal is also deposited. Thus, in equation (1.22), we can take metal $\mathrm{i}$ as the reactive metal. Then it is rewritten as:

$$
\operatorname{Eeq}(\operatorname{Rea})=E_{0}+\frac{R T}{z F} \ln \frac{\left[M e^{z+}\right]_{\mathrm{Re} a}}{a_{\mathrm{Re} a}^{\text {alloy }}}
$$

where the activity of $\mathrm{i}$ in solution is substituted by the reactive metal free ion concentration $\left[M e^{z+}\right]_{\operatorname{Re} a}$, and $a_{\operatorname{Re} a}^{\text {alloy }}$ is the activity of the reactive metal in the alloy.

Further, we can derive the activity of the reactive metal using the enthalpy of mixing:

$$
a_{\mathrm{Re} a}^{\text {alloy }}=x_{\mathrm{Re} a}^{\text {alloy }} \exp \left(\frac{\Delta H_{\text {Rea }}^{\text {partial }}}{R T}\right)
$$

where $x_{\mathrm{Rea}}^{\text {alloy }}$ is the atomic fraction of the reactive metal in the alloys, $\Delta H_{\text {Rea }}^{\text {partial }}$ the partial molar enthalpy of mixing for the reactive metal. 
After derivation combining equations (1.21), (1.23) and (1.24), (1.23) is rearranged as:

$$
E_{e q}(\operatorname{Re} a)=E_{0 .}+\frac{R T}{z F} \ln \frac{\left[M e^{z+}\right]_{\operatorname{Re} a}}{x_{\operatorname{Re} a}^{\text {alloy }} \exp \left(\frac{\left[W_{1}+2\left(W_{2}-W_{1}\right) x_{\operatorname{Re} a}^{\text {alloy }}\right]\left(1-x_{\mathrm{Re} a}^{\text {alloy }}\right)^{2}}{R T}\right)}
$$

As can be seen, the dependence of deposition on solid state thermodynamics can be simply described by the change of the equilibrium potential of the reactive metal, which is a function of the alloy composition. However, this thermodynamic behavior can only be followed when the system grows very close to thermodynamic equilibrium. In the following, we will discuss two types of systems with different solid state thermodynamic behaviors.

\subsubsection{Electrodeposition of solid solution type binary alloys}

In Fig. 1.4, it is shown a typical system $(\mathrm{Cu}-\mathrm{Au})$ with formation of solid solution for the whole composition range at low temperature, with several ordered structures at some compositions. The enthalpy of mixing is also shown here in Fig.1.5.

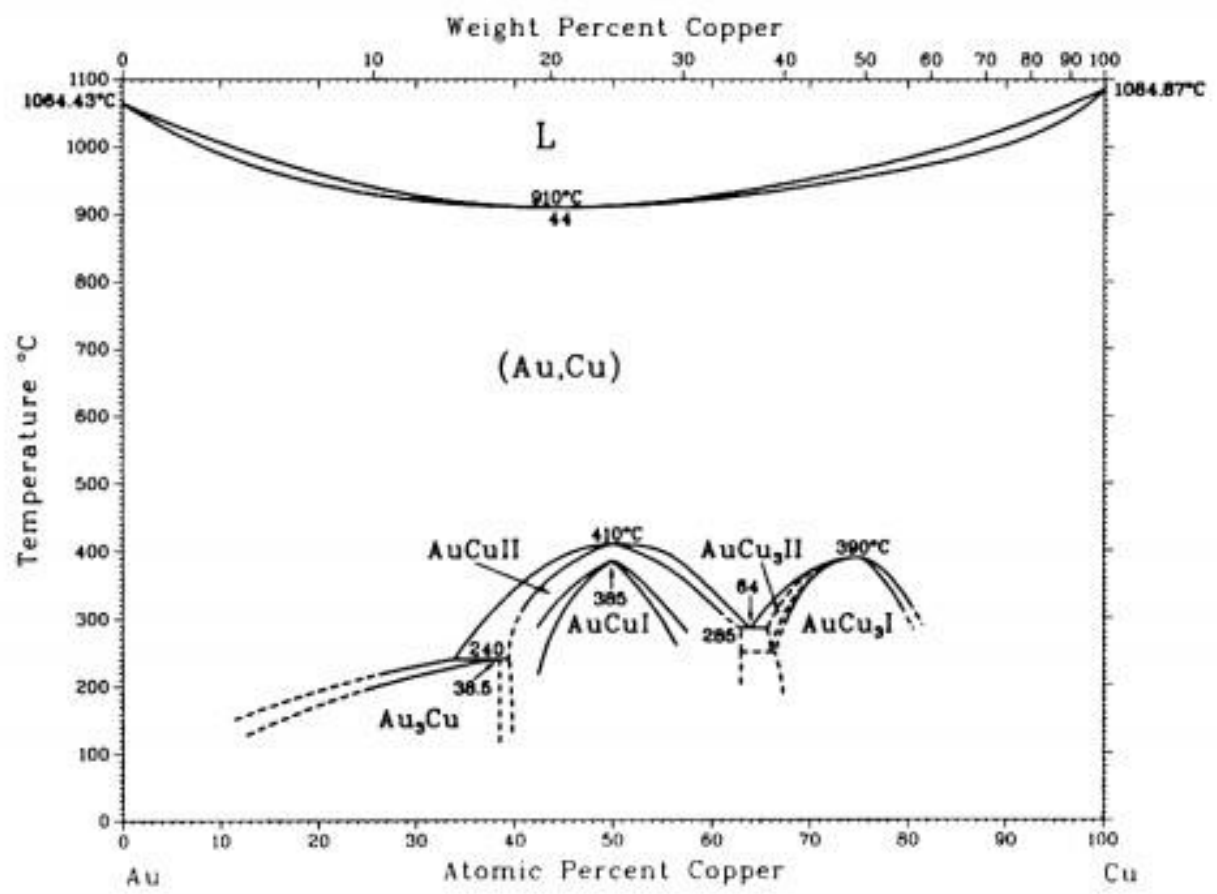

Figure 1.4 The equilibrium phase diagram of $\mathrm{Cu}-\mathrm{Au}$ system [15]. 


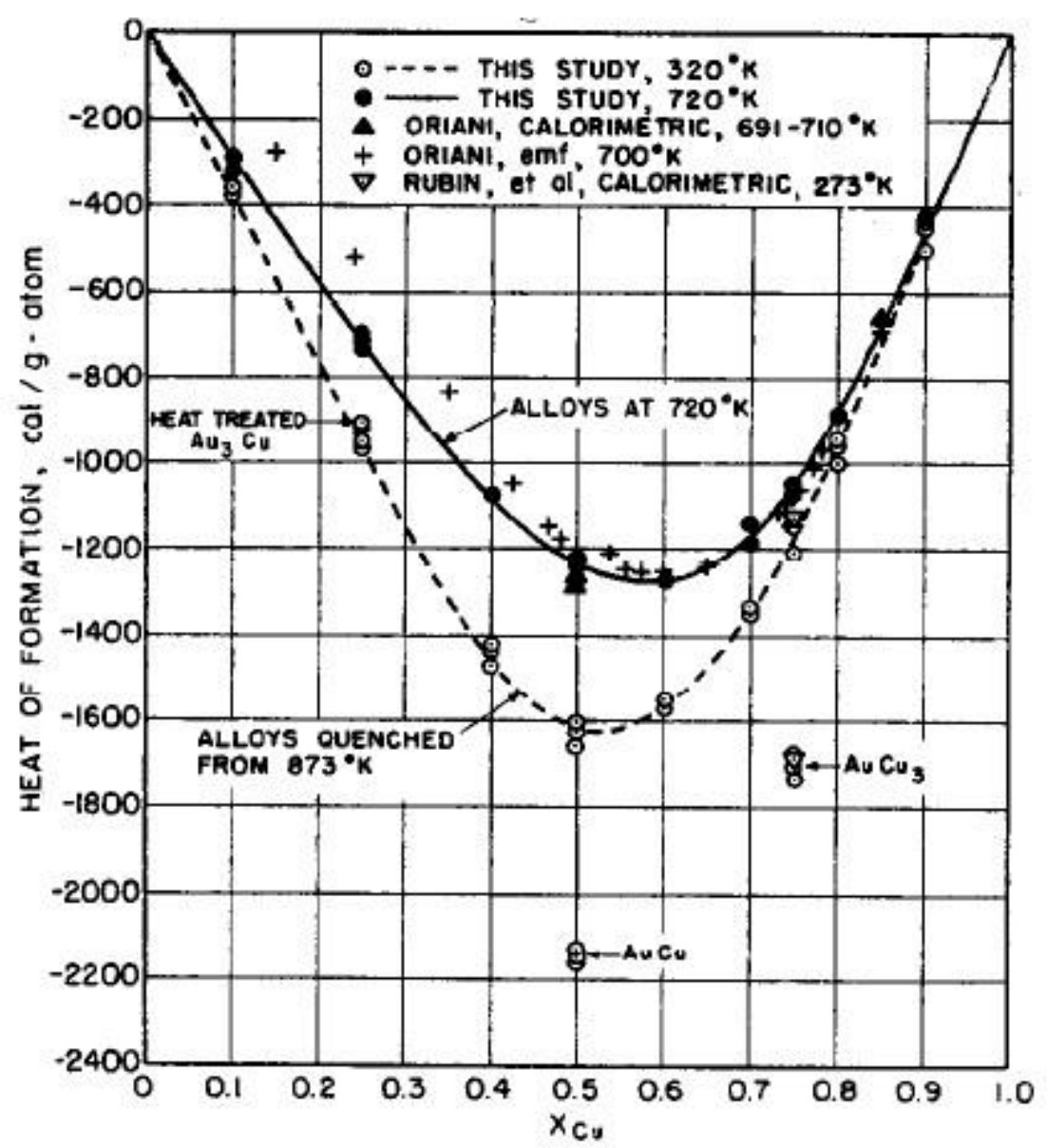

Figure 1.5 Enthalpy of mixing for Cu-Au system [16].

Using the experimental enthalpy data, the theoretical behavior for composition vs. potential can be predicted, assuming the formation of solid solution, as shown in Fig. 1.6. As shown in Fig.1.6, the overpotential $\left(\eta=E(i)-E_{e q}\right.$ as defined in equation (1.8)) is positive; in other words, $\mathrm{Cu}$ can be deposited at potentials more positive than its equilibrium potential. This is due to the formation of solid solution of $\mathrm{Cu}$ with $\mathrm{Au}$; $\mathrm{Au}-\mathrm{Cu}$ pairs are more favored than $\mathrm{Cu}-\mathrm{Cu}$ ones, resulting in the deposition of $\mathrm{Cu}$ at more positive potentials. The behavior, which leads to the reactive metal being deposited at more positive potentials with the noble metal than its corresponding equilibrium potential, is termed underpotential co-deposition (UPCD). The UPCD behavior in the $\mathrm{Au}-\mathrm{Cu}$ system has been confirmed by our group in previous study, where we showed that $\sim 9$ at. $\%$ of $\mathrm{Cu}$ was obtained in $\mathrm{Au}-\mathrm{Cu}$ alloys at $\sim 200 \mathrm{mV}$ positive than the equilibrium of $\mathrm{Cu}[17]$. 


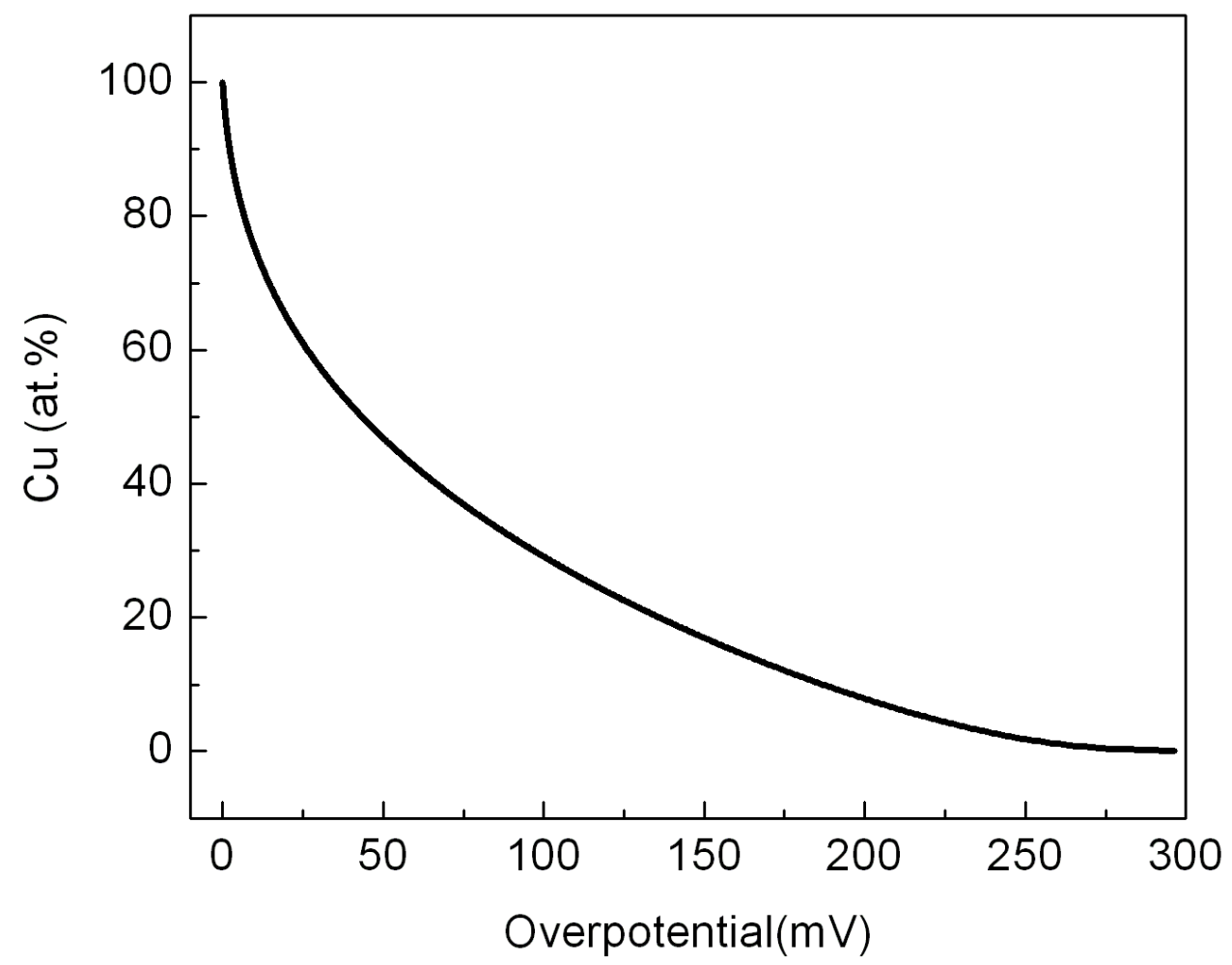

Figure 1.6 Theoretical composition vs. overpotential for $\mathrm{Au}-\mathrm{Cu}$ system, using the enthalpy data in Ref. [16].

\subsubsection{Electrodeposition of binary alloys with miscibility gap}

A phase diagram for such a system (Au-Ni) is shown in Fig. 1.7. Such systems are characterized by the existence of miscibility gap at low temperatures, either across the whole composition range or between certain compositions. For such systems, no shift in equilibrium potential is expected since they do not tend to form solid solutions; with the formation of separated phases, the activity of the reactive metal should be 1 at the case. However, if a metastable solid solution can form, e.g., by controlling deposition conditions, a negative shift of the reactive metal is expected. To be specific, the enthalpy of mixing at $1150 \mathrm{~K}$ (Fig. 1.8) is used for the theoretical prediction of the composition vs .potential for Au-Ni system. As shown in Fig. 1.9, Ni would be deposited at potentials more negative than its equilibrium potential upon the assumption of solid solution formation. 


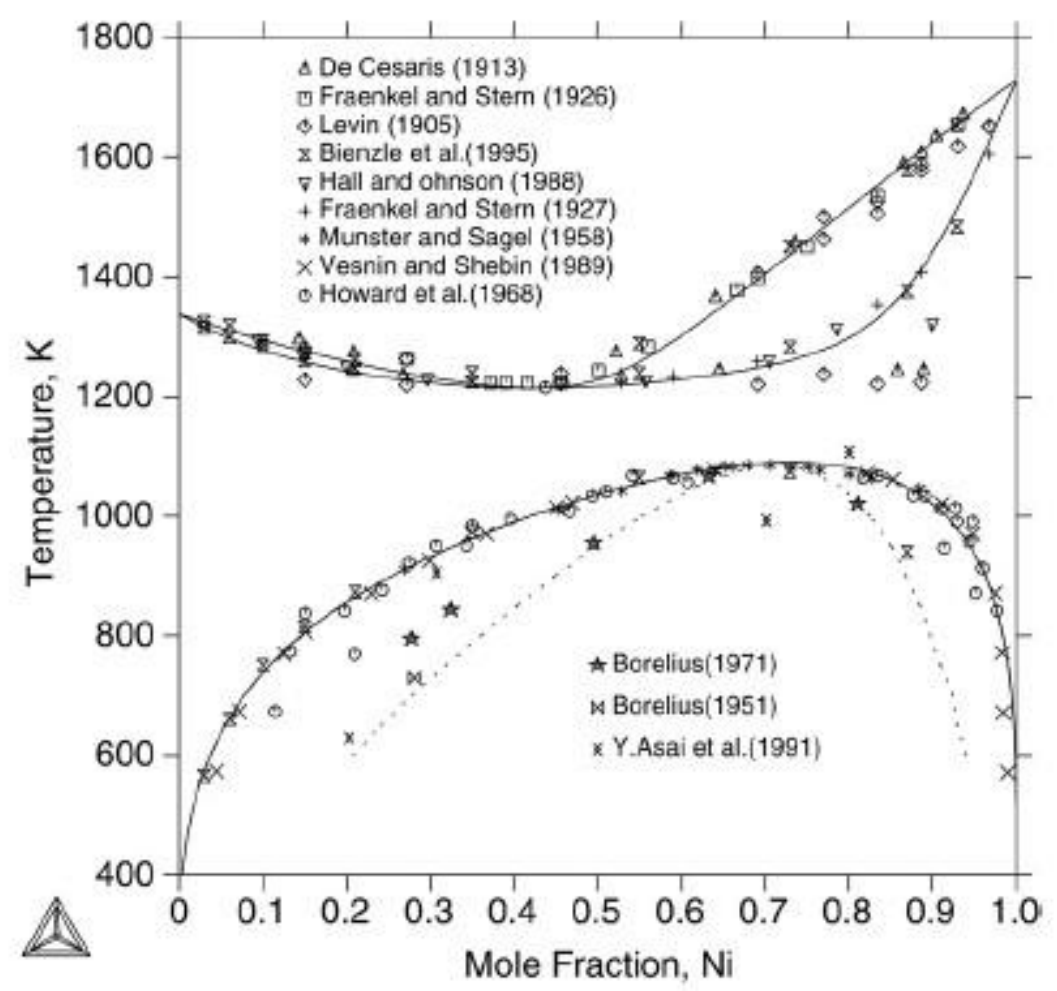

Figure 1.7 The equilibrium phase diagram of Au-Ni system [18].

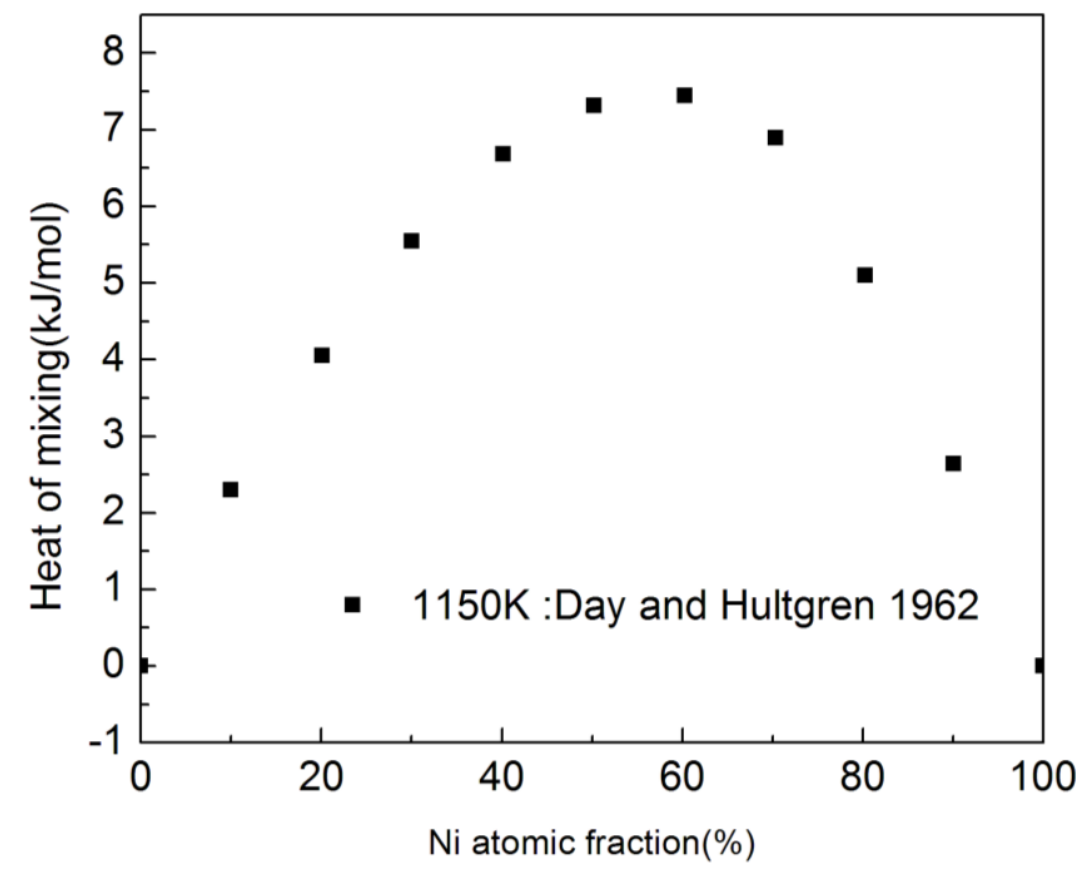

Figure 1.8 Enthalpy of mixing for Au-Ni at 1150K [19]. 


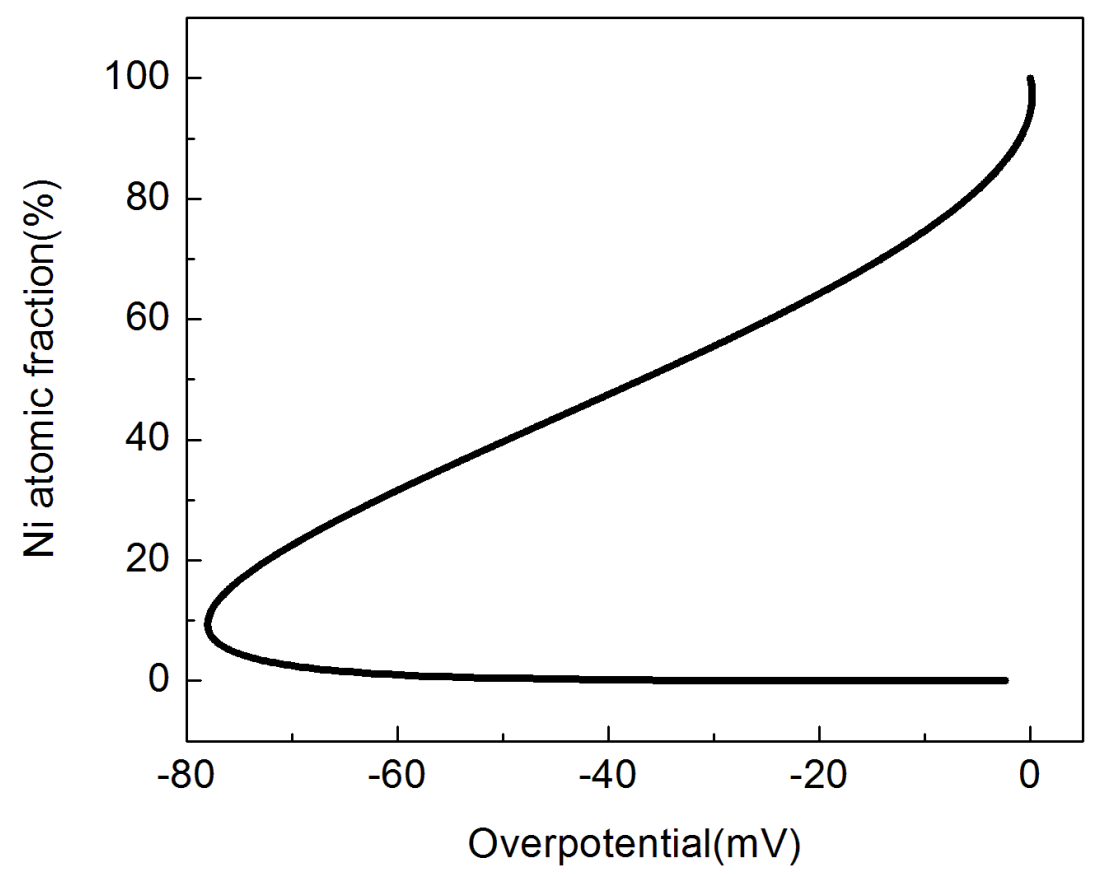

Figure 1.9 Theoretical composition vs. overpotential for Au-Ni system, using the enthalpy data in Ref. [19].

\subsection{Binary alloy systems studied in this dissertation}

As we have discussed in the Section of thermodynamics of electrodeposition, under thermodynamic limit (overpotential and deposition rate approach zero), tuning the applied potentials for electrodeposition allows the control of alloy composition, which is only dependent on thermodynamics and not on ion transport to the electrode. Experimental studies in our group supported this model for some systems. It will be very useful to get better understanding for this model: 1) can we extend it in a more general way to similar systems; 2) for practical applications, reasonable deposition rate is required, thus it is also very important to evaluate the limitation of thermodynamic assumption.

We will therefore study several alloy systems of fundamental and technological relevance. 


\subsection{1 $\mathrm{Cu}-\mathrm{Au}$ with bulk negative heat of mixing and no side hydrogen evolution reaction (HER) interference}

As discussed above, $\mathrm{Cu}-\mathrm{Au}$ alloy system forms solid solution at low temperatures, with three ordered structures $\mathrm{CuAu}_{3}, \mathrm{CuAu}$ and $\mathrm{Cu}_{3} \mathrm{Au}$. Meanwhile, both $\mathrm{Au}$ and $\mathrm{Cu}$ can be electrodeposited without the usual parasitic HER, making it a prototype system for electrochemical study, e.g., Cu UPD on Au $[11,20]$. We will study deposition from a simple solution, which yields powdery deposits; deposition from complexing solution will then be studied, from which we will show that deposits with greatly improved morphology are achieved.

\subsubsection{Fe-Pt with bulk negative heat of mixing}

The Fe-Pt alloy system has a large negative heat of mixing and its phase diagram presents both ordered phases and solid solutions (Fig. 1.13). At room temperature, three ordered structures with the composition $\mathrm{FePt}_{3}, \mathrm{FePt}$ and $\mathrm{Fe}_{3} \mathrm{Pt}$ can be formed [21]. Fe-Pt alloys are intensively investigated due to their unique magnetic [22] and

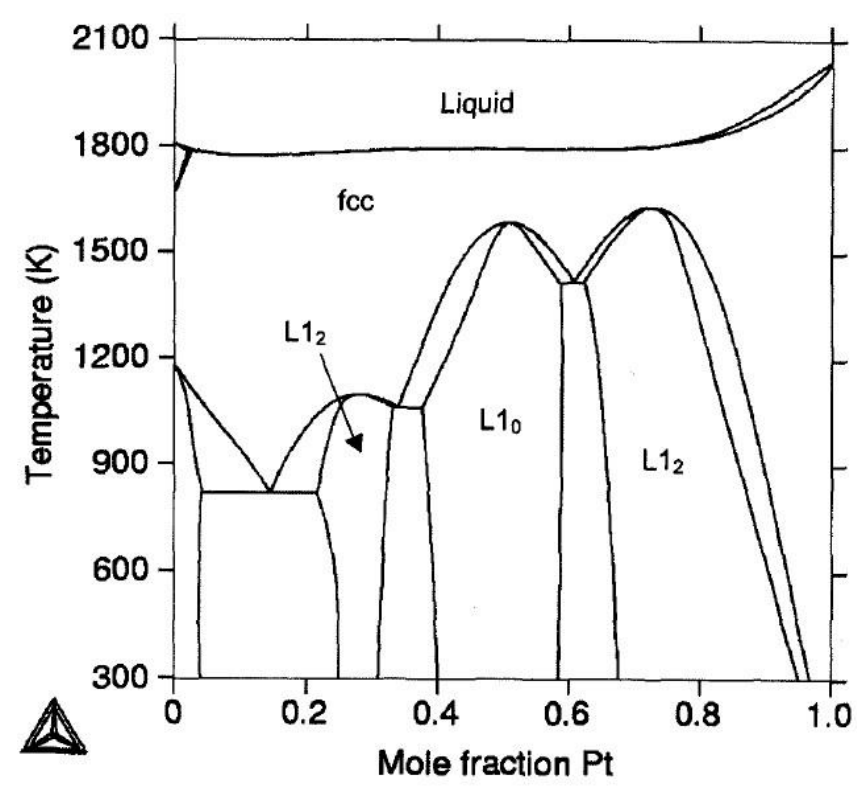

Figure 1.13 Equilibrium phase diagram of Fe-Pt system [23].

catalytic properties and chemical stability [24]. The high magnetic anisotropy 
$\left(10^{7} \mathrm{MJ} / \mathrm{m}^{3}\right)$ of the near equiatomic alloys with the ordered $\mathrm{L} 1_{0}$ structure is particularly interesting for magnetic recording media applications [25].

\subsubsection{Au-Ni with bulk positive heat of mixing}

Au-Ni alloys have been recently extensively researched due to their unique physical, chemical and mechanical properties and therefore potential applications in electrical contact and switching materials [26], magnetic drug delivery [27] and catalyst for CO oxidation [28]. Bulk Au-Ni can form face centered cubic (FCC) solid solutions in the whole composition range above $\sim 1090 \mathrm{~K}$; at lower temperatures, there is a large immiscible region containing phase separated FCC Au and FCC Ni, due to the large atomic volume difference [24] (as shown in Fig. 1.7). This system is interesting here, partially due to the fact that Au-Ni pairs are favored than $\mathrm{Au}-\mathrm{Au}$ and $\mathrm{Ni}-\mathrm{Ni}$ pairs; the miscibility gap at low temperature is a result of large strain energy due to the large difference in atomic volumes of $\mathrm{Au}$ and $\mathrm{Ni}$ atoms.

\subsubsection{Ag-Cu with bulk positive heat of mixing and a eutectic alloy system}

$\mathrm{Ag}-\mathrm{Cu}$ shows a eutectic phase diagram with negligible solubility of either element in the crystal structure of the other one at room temperature, due to the repulsive interactions and the difference in atomic sizes (Fig. 1.14). The liquids are miscible[30,31]. 


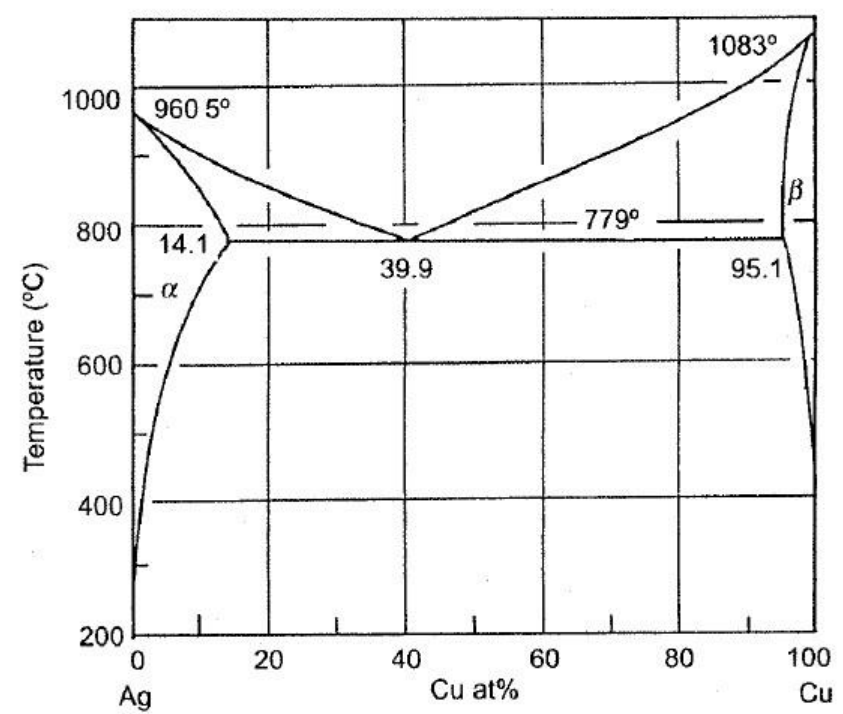

Figure 1.14 Equilibrium phase diagram of $\mathrm{Ag}-\mathrm{Cu}$ system [32].

\subsubsection{Ag-Ni with bulk positive heat of mixing}

$\mathrm{Ag}-\mathrm{Ni}$ system is somewhat similar to $\mathrm{Au}-\mathrm{Ni}$, but at variance with the latter is that it shows a miscibility gap even in the liquid state [33] (Fig. 1.15); the solubility of Ni in $\mathrm{Ag}$ for example is at most 0.0219 at. $\%$ at $400^{\circ} \mathrm{C}$ [34]. $\mathrm{Ag}-\mathrm{Ni}$ alloys or bimetallic structures are of technological interest due to their remarkable catalytic [35], magnetic [36], and electrical [37] properties. In particular, Ag-Ni alloys are promising for electrical contacts and switches applications [34], where they could provide a low cost version of the widely employed hard gold alloys, currently the material of choice for commercial products [38]. More recently, nanostructured Ag-Ni with nanoscale ferromagnetic $\mathrm{Ni}$ grains embedded in a non-magnetic $\mathrm{Ag}$ matrix has attracted considerable attention as a potential giant magnetoresistance (GMR) material [39]. 


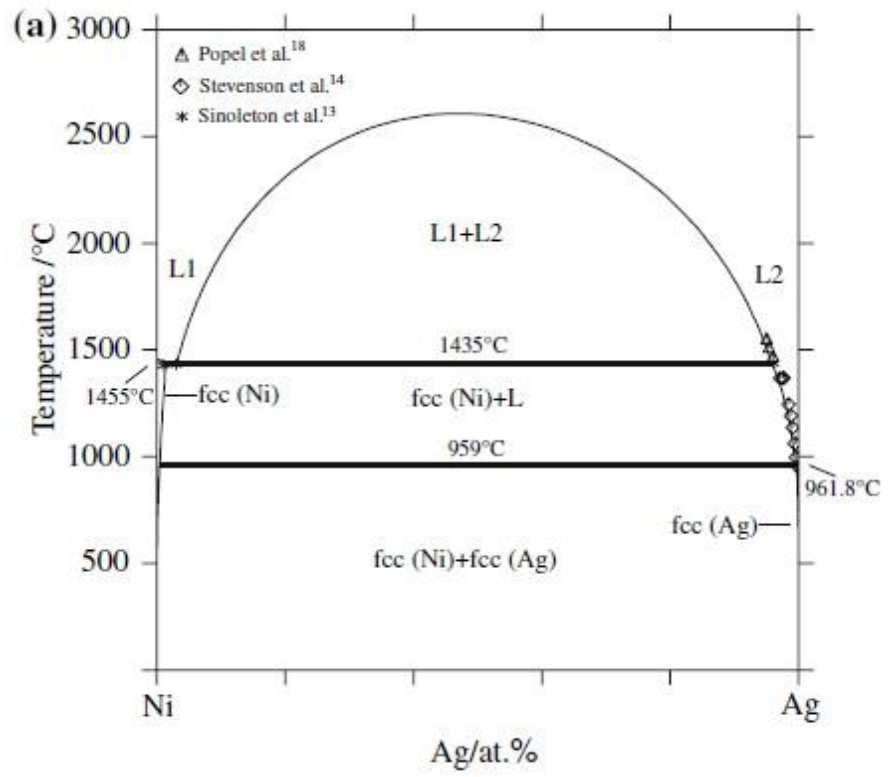

Figure 1.15 Equilibrium phase diagram of Ag-Ni system [33].

In the following, the thermodynamics of electrodeposition of these alloy systems and corresponding properties of the deposits will be studied in detail. 


\section{References}

[1] A.K. Agrawal, J. Electrochem. Soc. 128 (1981) 2292.

[2] T.J. Zhao, Yuguang, VanderNoot, Electrochimi. Acta 42 (1997) 3.

[3] G.J. Hills, D.J. Schiffrin, J. Thompson, Electrochimi. Acta 19 (1974) 657.

[4] P. Taxil, P. Chamelot, L. Massot, C. Hamel, J. Min. Metall. Sect. B. 39 (2003) 177.

[5] F. Endres, D. MacFarlane, A. Abbott, eds., Electrodeposition from Ionic Liquids, 1st ed., John Wiley \& Sons, Inc., 2008.

[6] D.. Kolb, Surf. Sci. 500 (2002) 722.

[7] M. Paunovic, M. Schlesinger, Fundamentals of Electrochemical Deposition, 2nd ed., John Wiley and Sons, New York, 2006.

[8] E. Budevski, G. Staikov, W.J. Lorenz, Electrochemical Phase Formation and Growth: An Introduction to the Initial Stages of Metal Deposition, New York, 1996.

[9] J.L. Barton, J.O. Bockris, Proc. R. Soc. London, Ser. A 268 (1962) 485.

[10] D.M. Kolb, M. Przasnyski, H. Gerischer, J. Electroanal. Chem. Interfacial Electrochem. 54 (1974) 25.

[11] E. Herrero, L.J. Buller, H.D. Abruña, Chem. Rev. 101 (2001) 1897.

[12] M. Asta, S. Foiles, Phys. Rev. B 53 (1996) 2389.

[13] Y. Liu, D. Gokcen, U. Bertocci, T.P. Moffat, Science 338 (2012) 1327.

[14] H.K. Hardy, Acta Metall. 1 (1953) 202.

[15] H. Okamoto, D.J. Chakrabarti, D.E. Laughlin, T.B. Massalski, Bull. Alloy Phase Diagrams 8 (1987) 454.

[16] R.L. Orr, Acta Metall. 8 (1960) 489.

[17] J.J. Mallett, W. Shao, D. Liang, G. Zangari, Electrochem. Solid-State Lett. 12 (2009) D57.

[18] J. Wang, X. Lu, B. Sundman, X. Su, Calphad 29 (2005) 263. 
[19] G.F. Day, R. Hultgren, J. Phys. Chem. 66 (1962) 1532.

[20] T. Hachiya, H. Honbo, K. Itaya, J. Electroanal. Chem. 315 (1991) 275.

[21] P. Fredriksson, Calphad 25 (2001) 535-548.

[22] S. Sun, Science 287 (2000) 1989.

[23] P. Fredriksson, B. Sundman, Calphad 25 (2001) 535.

[24] J. Kim, Y. Lee, S. Sun, J. Am. Chem. Soc. 132 (2010) 4996.

[25] C.A. Ross, Annu. Rev. Mater. Res. 31 (2001) 203.

[26] A. Dolati, M. Ghorbani, M.R. Ahmadi, J. Electroanal. Chem. 577 (2005) 1.

[27] P.C. Andricacos, C. Uzoh, J.O. Dukovic, J. Horkans, H. Deligianni, IBM J. Res. Dev. 42 (1998) 567.

[28] A.M. Molenbroek, J.K. Nørskov, B.S. Clausen, J. Phys. Chem. B 105 (2001) $5450-5458$.

[29] I.B. Ramsteiner, V.N. Bugaev, O. Shchyglo, A. Udyansky, H. Dosch, M. Asta, H. Reichert, A. Scho, 235703 (2005) 2-5.

[30] V. Moreno, J. Creuze, F. Berthier, C. Mottet, G. Treglia, B. Legrand, Surf. Sci. 600 (2006) 5011-5020.

[31] P.L. Williams, Y. Mishin, J.C. Hamilton, Modell. Simul. Mater. Sci. Eng. 14 (2006) 817.

[32] T.B. Massalski, Binary Alloy Phase Diagrams, 2nd ed., ASM International, 1990.

[33] X.J. Liu, F. Gao, C.P. Wang, K. Ishida, J. Electron. Mater. 37 (2007) 210.

[34] E.H. Tyler, J.R. Clinton, H.L. Luo, Solid State Commun. 13 (1973) 1409.

[35] H. Guo, Y. Chen, X. Chen, R. Wen, G.-H. Yue, D.-L. Peng, Nanotechnol. 22 (2011) 195604.

[36] O. Proux, J. Mimault, C. Revenant-Brizard, J.R. Regnard, B. Mevel, J. Phys. Condens. Matter. 11 (1999) 147.

[37] J. Swingler, IET Sci. Meas. Technol. 5 (2011) 37. 
[38] Y. Okinaka, Gold Bull. 31 (1998) 3.

[39] L.B. Barthélémy, A., Cros, V., Duvail, J.L., Fert, A., Morel, R., Parent, F., Petroff, F., Steren, Nanostruct. Mater. 6 (1995) 217.

[1] A.K. Agrawal, J. Electrochem. Soc. 128 (1981) 2292.

[2] T.J. Zhao, Yuguang, VanderNoot, Electrochimi. Acta 42 (1997) 3.

[3] G.J. Hills, D.J. Schiffrin, J. Thompson, Electrochimi. Acta 19 (1974) 657.

[4] P. Taxil, P. Chamelot, L. Massot, C. Hamel, J. Min. Metall. Sect. B. 39 (2003) 177.

[5] F. Endres, D. MacFarlane, A. Abbott, eds., Electrodeposition from Ionic Liquids, 1st ed., John Wiley \& Sons, Inc., 2008.

[6] D.. Kolb, Surf. Sci. 500 (2002) 722.

[7] M. Paunovic, M. Schlesinger, Fundamentals of Electrochemical Deposition, 2nd ed., John Wiley and Sons, New York, 2006.

[8] E. Budevski, G. Staikov, W.J. Lorenz, Electrochemical Phase Formation and Growth: An Introduction to the Initial Stages of Metal Deposition, New York, 1996.

[9] J.L. Barton, J.O. Bockris, Proc. R. Soc. London, Ser. A 268 (1962) 485.

[10] D.M. Kolb, M. Przasnyski, H. Gerischer, J. Electroanal. Chem. Interfacial Electrochem. 54 (1974) 25.

[11] E. Herrero, L.J. Buller, H.D. Abruña, Chem. Rev. 101 (2001) 1897.

[12] M. Asta, S. Foiles, Phys. Rev. B 53 (1996) 2389.

[13] Y. Liu, D. Gokcen, U. Bertocci, T.P. Moffat, Science 338 (2012) 1327.

[14] H.K. Hardy, Acta Metall. 1 (1953) 202.

[15] H. Okamoto, D.J. Chakrabarti, D.E. Laughlin, T.B. Massalski, Bull. Alloy Phase Diagrams 8 (1987) 454.

[16] R.L. Orr, Acta Metall. 8 (1960) 489.

[17] J.J. Mallett, W. Shao, D. Liang, G. Zangari, Electrochem. Solid-State Lett. 12 (2009) D57. 
[18] J. Wang, X. Lu, B. Sundman, X. Su, Calphad 29 (2005) 263.

[19] G.F. Day, R. Hultgren, J. Phys. Chem. 66 (1962) 1532.

[20] T. Hachiya, H. Honbo, K. Itaya, J. Electroanal. Chem. 315 (1991) 275.

[21] P. Fredriksson, Calphad 25 (2001) 535-548.

[22] S. Sun, Science 287 (2000) 1989.

[23] P. Fredriksson, B. Sundman, Calphad 25 (2001) 535.

[24] J. Kim, Y. Lee, S. Sun, J. Am. Chem. Soc. 132 (2010) 4996.

[25] C.A. Ross, Annu. Rev. Mater. Res. 31 (2001) 203.

[26] A. Dolati, M. Ghorbani, M.R. Ahmadi, J. Electroanal. Chem. 577 (2005) 1.

[27] P.C. Andricacos, C. Uzoh, J.O. Dukovic, J. Horkans, H. Deligianni, IBM J. Res. Dev. 42 (1998) 567.

[28] A.M. Molenbroek, J.K. Nørskov, B.S. Clausen, J. Phys. Chem. B 105 (2001) $5450-5458$

[29] I.B. Ramsteiner, V.N. Bugaev, O. Shchyglo, A. Udyansky, H. Dosch, M. Asta, H. Reichert, A. Scho, 235703 (2005) 2-5.

[30] V. Moreno, J. Creuze, F. Berthier, C. Mottet, G. Treglia, B. Legrand, Surf. Sci. 600 (2006) 5011-5020.

[31] P.L. Williams, Y. Mishin, J.C. Hamilton, Modell. Simul. Mater. Sci. Eng. 14 (2006) 817.

[32] T.B. Massalski, Binary Alloy Phase Diagrams, 2nd ed., ASM International, 1990.

[33] X.J. Liu, F. Gao, C.P. Wang, K. Ishida, J. Electron. Mater. 37 (2007) 210.

[34] E.H. Tyler, J.R. Clinton, H.L. Luo, Solid State Commun. 13 (1973) 1409.

[35] H. Guo, Y. Chen, X. Chen, R. Wen, G.-H. Yue, D.-L. Peng, Nanotechnol. 22 (2011) 195604.

[36] O. Proux, J. Mimault, C. Revenant-Brizard, J.R. Regnard, B. Mevel, J. Phys. Condens. Matter. 11 (1999) 147. 
[37] J. Swingler, IET Sci. Meas. Technol. 5 (2011) 37.

[38] Y. Okinaka, Gold Bull. 31 (1998) 3.

[39] L.B. Barthélémy, A., Cros, V., Duvail, J.L., Fert, A., Morel, R., Parent, F., Petroff, F., Steren, Nanostruct. Mater. 6 (1995) 217. 


\section{Chapter 2 Methods and Procedures}

\section{Overview}

In this chapter, the methods and equipments used for the deposition and characterization of alloy films will be briefly discussed. Since this dissertation describes several alloy systems, the details, e.g., about solutions, substrates and thermal annealing will be addressed for each system in later chapters correspondingly. This chapter will be divided into two parts: part I, methods and equipments for electrochemical studies; Part II, alloy characterization: composition, morphology, thickness, microstructure, crystal structure and magnetic properties.

\subsection{Electrochemical Characterization}

\subsubsection{Cyclic Voltammetry (CV)}

The electrochemical behavior of electrolytes for alloy deposition is characterized by CV. Substrates used are immersed into the solutions, then the potential is scanned, usually starting from open circuit potential (OCP), first go in the negative (cathodic) direction to a preset potential and then back to a second predetermined potential. The current is recorded as a function of the scanning potential. From the E (j) vs. $j$ behavior, general information, such as onset of deposition, etching and hydrogen generation may be assessed.

\subsubsection{Electrochemical Quartz Crystal Microbalance (EQCM)}

$\mathrm{CV}$ can only record the current density as a function of potential. When significant side reactions occur, e.g., hydrogen evolution in parallel with the reactions of interest, CV lacks the ability to separate the currents due to different reactions. In this respect, EQCM is a very important technique to measure the mass change occurring on the working electrode real-time and in-situ. What's more, EQCM can be coupled with 
other electrochemical techniques, here $\mathrm{CV}$, to simultaneously obtain additional information. In this dissertation, an EQCM (EQCM, model QCM922, Princeton Applied Research) was used together with EG\&G Model 283 Potentiostat / Galvanostat (Princeton Applied Research) to record current density and mass changes at the electrode as a function of the applied potential. This enables the separation of the current used up in metal or alloy deposition from that of side reactions, thus determining the actual onset of metal deposition as well as the cathodic efficiency of the process. A schematic representation for the EQCM is shown in Fig. 2.1.

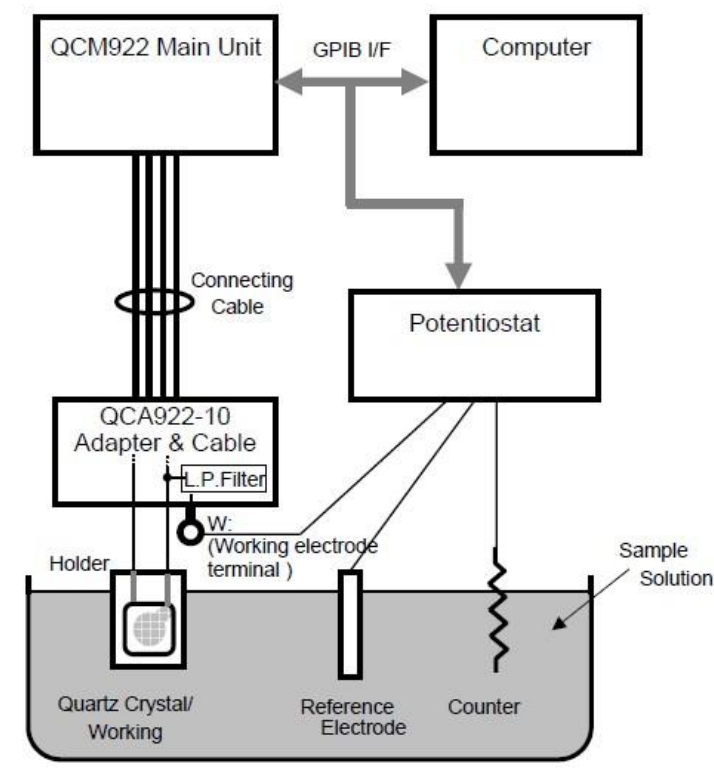

Figure 2.1 An EQCM setup scheme [1].

The working electrode for the EQCM was a Au coated AT-cut quartz resonator with $0.5 \mathrm{~cm}$ diameter $\left(0.196 \mathrm{~cm}^{2}\right.$ working area) and a resonant frequency of $9 \mathrm{MHz}$. The output of the EQCM is the change in frequency $\Delta f$ of the Au resonator during the potential scan, which directly provides the change in mass $\Delta m$ through the Sauerbrey equation [2]. Substitution of the known quartz crystal constants in the Sauerbrey formula yields the change of mass as a function of frequency

$$
\Delta m(n g)=-1.068 \times \Delta f(H z)
$$

The partial current for single metal ion reduction was calculated from the rate of change in $\mathrm{m},(\mathrm{dm} / \mathrm{dt})$ expt., using the following formula: 


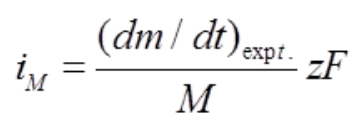

where $\mathrm{M}$ is the atomic weight of the deposited species, $\mathrm{z}$ the number of electrons transferred during metal reduction, and F Faraday's constant. The ratio $i_{M} / i_{\exp }$ gives an approximate current efficiency for single metal deposition. All solutions for EQCM characterization were deaerated by purging nitrogen gas for about 30mins before measurement; corresponding $\mathrm{CV}$ curves were collected at various scan rates, which will be detailed for each system later.

\subsection{Materials Characterization}

\subsubsection{Morphology and composition}

In this section, techniques and equipment for morphology and composition analysis will be introduced: Scanning electron microscope, energy-dispersive X-ray spectroscope, Auger electron spectroscopy, X-ray photoelectron spectroscopy and atomic force microscope.

\subsubsection{Scanning Electron Microscope (SEM)}

In the SEM, a source of electrons is focused into a fine probe, which is then rastered over the surface of the sample for measurement. Various interactions between the incident electrons and the sample occur, resulting in the emission of electrons or photons from the sample surface, including secondary electrons and backscattered electrons. Appropriate detectors are used to collect different types of emitted electrons; a certain amount of emitted electrons can be collected and the output is used to modulate the brightness of a cathode ray tube (CRT) whose $\mathrm{x}$ - and $\mathrm{y}$ - inputs are driven in synchronism with the $x-y$ voltages rastering the electron beam. An image is formed in the CRT, with each point the beam strikes directly mapped onto a corresponding point in the screen. A schematic depiction of a SEM is shown in Fig. 


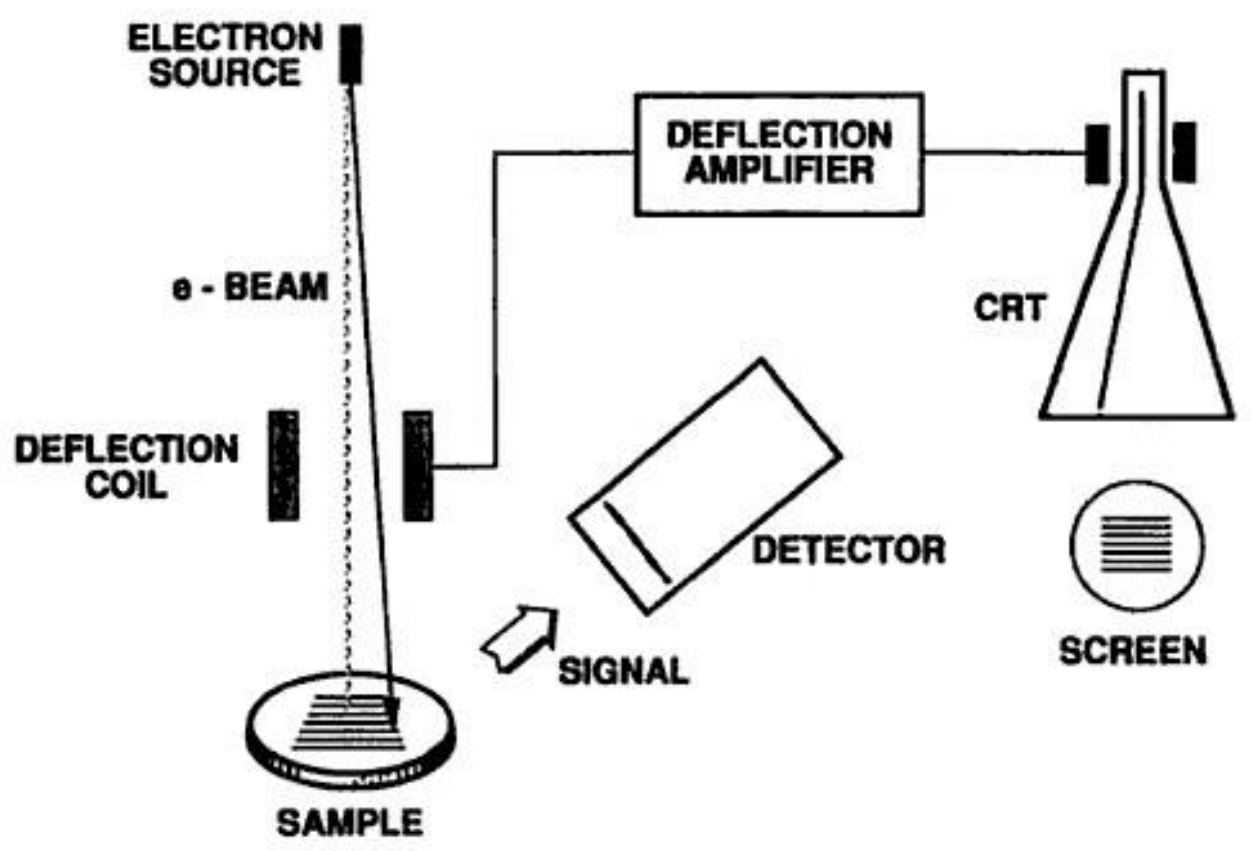

Figure 2.2 A schematic showing the operation of SEM [3].

\subsubsection{Energy-Dispersive X-ray Spectroscopy (EDS)}

EDS analyzes the chemical composition by collecting the characteristic X-rays emitted from various elements presented in the specimen. These X-rays are generated from the ionization of the element atom by high energy radiation: an inner shell electron is removed, and then another electron in a higher energy outer shell will fill the available shell, releasing an amount of energy equal to the energy difference between these two shells. The energy of the X-ray thus emitted is characteristic of a certain transition in each element. A specific name is given to each transition: an L shell to $\mathrm{K}$ shell is named $K_{\alpha}$, an $\mathrm{M}$ shell to $\mathrm{K}$ shell $K_{\beta}$, an $\mathrm{M}$ shell to $\mathrm{L}$ shell $L_{\alpha}$, and so on.

Due to the high energy of the incident electrons (level of keV), X-ray is stimulated from several $\mu m$ thick region. As a result, this method provides the average 
composition within a relatively deep region. The accuracy of EDS is limited also due to the overlapping of the X-rays from different elements; as a result, an error of $\pm 4-5 \%$ is usually expected, even with standard sample calibration [3].

EDS instruments are usually attached to the electron column instruments such as SEM and transmission electron microscope (TEM). The advantage of EDS lies in the fact that it is convenient to operate; it takes only several minutes to collect spectrum and obtain the composition information.

\subsubsection{X-ray photoelectron spectroscopy (XPS)}

The XPS technique was developed according to the fact that the electron binding energy is unique and also sensitive to its chemical environment. It uses x-ray beam with sufficient energy (usually $3-10 \mathrm{keV}$ ); an $\mathrm{x}$-ray hits and ionizes an atom, generating an ejected electron, the kinetic energy $K E$ of which depends on the energy of the incident photon $\hbar v$ and the binding energy $B E$ of the particular electron to the atom involved:

$$
K E=\hbar v-B E
$$

The core shell electron energy level is nearly independent of the chemical species to which the atom is bound, thus it provides the identification of the elements and the chemical composition analysis for the sample. On the other hand, though the $B E$ is approximately constant for an atom in various chemical environments, it is not exactly constant; the general trend is that the lower the valence electron charge due to the chemical bond (for example, oxidation), the higher the $B E$, e.g., $\mathrm{Ni}$ in $\mathrm{NiO}$ with $\mathrm{Ni}^{2+}$ is higher in $\mathrm{BE}$ than $\mathrm{Ni}$ metal with $\mathrm{Ni}^{0}[3]$. As a result, XPS can also provide information about the chemical state of the involved atom.

The ejected electrons in XPS can travel only short distances through the solid before losing energy in collisions with the atoms in the sample. It is therefore a surface sensitive technique. Combined with ion sputtering techniques, XPS is able to provide 
the elemental and chemical state depth profile.

\subsubsection{Auger electron spectroscopy (AES)}

In AES, an electron beam is used to bombard the sample, generating an empty shell with the removal of an inner shell electron. Similar to the XPS process, a second atomic electron from a higher shell will fill this vacancy. Contrast with XPS, the excess energy therefore produced is carried by a third electron, a so-called Auger electron, in a radiationless process; this process is termed the Auger process. Since the Auger emission involves specific energy levels, which are characteristic of each atom, the Auger spectra obtained can be used for elemental analysis [4]. AES used for the study is a Perkin-Elmer PHI 660 Scanning Auger Multiprobe.

\subsubsection{Atomic Force Microscope (AFM)}

The morphology of the films can also be studied with AFM. The working principle of AFM is based on the deflection of a very sensitive cantilever, which results from the repulsive forces between atoms at the cantilever tip and on the specimen surface. A piezoelectric tube is used to control the motion when the tip is rastered over the sample. The deflection is monitored by a laser beam at the same time as a function of the sample position; the surface morphology then is reconstructed using suitable software. There are usually two working modes available for AFM: contact mode, in which the cantilever moves across and in continuous contact with the sample surface at constant force; or tapping mode, in which the cantilever oscillates up and down at near its resonance frequency, leading to the intermittent contact of the tip with the sample surface. Due to the fact that the radius of the tip is several tens of nms, AFM may be unable to detect the features of samples with smaller structures.

The instrument used was a Digital Instruments, Dimension 3100, utilized in tapping 
mode for experiments in the thesis.

\subsubsection{Crystal structure and microstructure}

\subsubsection{X-ray Diffraction (XRD)}

XRD is a standard technique for the determination of crystal structures. It is a non destructive method and requires little sample preparation; with modern state -in- art models, structural information can be obtained in minutes.

The working principle of XRD is based on the interaction between an incident X-ray beam and the electron cloud of the sample atoms. A schematic of a typical XRD experiment setup is shown in Fig. 2.3.

The most common configuration is the so-called $\theta-2 \theta$ (Bragg- Brentano) geometry setup. The angle between the incident $\mathrm{x}$-rays and the diffracted $\mathrm{x}$-rays changes continuously during scan while keeping then incident and diffraction angles the same, and the intensity of the diffracted $\mathrm{x}$-rays is recorded at the same time by the detector. At some specific angles, which are corresponding and characteristic of the examined sample, the constructive interference is satisfied; an increase in the intensity of diffracted $\mathrm{x}$-rays occurs and a diffraction peak is observed. 


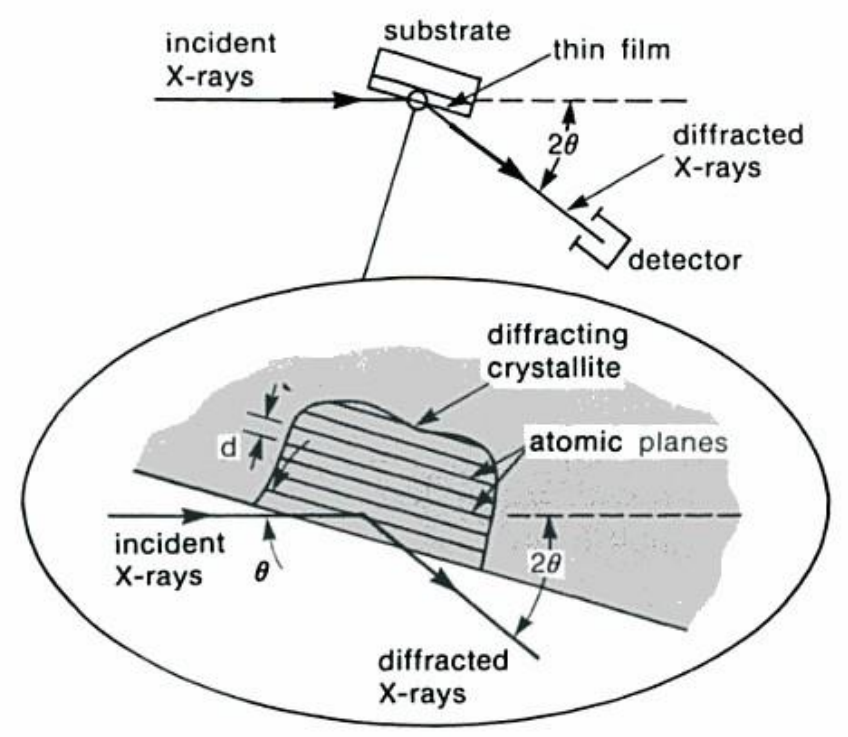

Figure 2.3 Basic features of a typical XRD setup [3].

The conditions for constructive interference can be assessed by the Bragg's law:

$$
2 d_{h k l} \sin \theta_{h k l}=\lambda
$$

where (hkl) are the miller indices of the crystallographic plane under consideration, $d_{h k l}$ the plane spacing of (hkl) planes, $\theta_{h k l}$ the angle between the incident x-rays and the atomic planes, $\lambda$ the wavelength of the incident $\mathrm{x}$-rays, which is a known parameter of the equipment.

The spacing of (hkl) planes can be calculated using known crystallographic parameters of the studied systems. Take an orthorhombic structure for example,

$$
d_{h k l}=\frac{1}{\sqrt{\frac{h^{2}}{a^{2}}+\frac{k^{2}}{b^{2}}+\frac{l^{2}}{c^{2}}}}
$$

where $(\mathrm{a} b \mathrm{c})$ are the lattice constants in the three orthorhombic directions. Further, for a cubic structure where $a=b=c$, equation (2.5) can be simplified as:

$$
d_{h k l}=\frac{a}{\sqrt{h^{2}+k^{2}+l^{2}}}
$$

Different materials systems usually have different lattice constants; a set of diffraction 
peaks can therefore be used as the identification of the phase structures.

By simply comparing the intensities of different diffraction peaks, the crystallographic orientation of the system can be assessed. When there is an evident stronger peak, this is termed preferential orientation. For example, a face centered cubic structure usually shows (111) orientation.

More information, such as grain size, defect structures and strain state can be examined using XRD.

The instruments used for this study are a model XDS 2000 (Scintag Inc.) with Cu Ka radiation $(\lambda=1.5406 \AA)$ and Panalytical X'Pert diffractomer with $\mathrm{Cu} \mathrm{K} \alpha$ radiation $(\lambda=1.5418 \AA)$.

\subsubsection{Transmission electron microscope (TEM)}

In $\mathrm{XRD}$, the information obtained comes from a region with size of several $\mu \mathrm{m}$ lacking thus in spatial resolution. This is especially significant when working with alloy systems for which composition and structure homogeneity are of interest and importance. TEM in this respect provides high lateral spatial resolution with both image and diffraction information from a single sample. It is however a destructive method and requires laborious sample preparation compared with XRD.

In TEM, the acceleration voltage is very high, usually $80 \mathrm{kV}$ to $300 \mathrm{kV}$, generating a highly focused coherent electron beam of monoenergetic electrons of exceedingly small wavelength. The focused beam is incident on a thin (less than $200 \mathrm{~nm}$, and for high resolution TEM, usually $\sim 20 \mathrm{~nm}$ ) specimen. Then the beam is controlled by a series of magnetic lenses above and below the sample to deliver the signal, undeflected and deflected electrons, to a detector, a fluorescent screen or a camera. The electrons in the beam are in phase as they enter the specimen; their phase 
relationships when exiting the sample are correlated to the spatial correlation between scattering centers (atoms) inside the material [3]. The obtained magnification ranges from as low as 50 times to as high as a factor of $10^{6}$.

TEM operation includes two methods, diffraction mode and image mode. In diffraction mode, the working principle is equivalent to that of x-ray diffraction; TEM diffraction patterns can be directly compared to the patterns obtained by XRD: a single crystal shows a spot pattern, polycrystalline materials generate ring patterns and amorphous samples produce a series of diffuse halos.

The Image mode provides an image of the illuminated sample area. The contrast in images comes from several mechanisms: thickness contrast due to nonuniformity in thickness, mass contrast due to spatial separations between distinct atomic constituents, diffraction contrast and phase contrast. In image mode, according to the modality of use of the objective diaphagram as a filter in the back focal plane, three main image modes are commonly used in TEM: bright-field (BF) microscopy, dark-field (DF) microscopy and high resolution (HRTEM) microscopy.

In BF mode, a small objective aperture is used to block out all the diffracted signals, allowing only the transmitted electron beam. In contrast, in DF mode, the aperture helps to block the transmitted beam and passes the diffracted signals. BF and DF modes in combination can provide more details on the microstructure of the sample.

A method based on DF mode is used to estimate the transformed phase fraction in annealing samples [5,6]. In the diffraction mode, the objective aperture is placed so as to select some diffracted beams to pass while at the same time blocking the transmitted beam and other diffraction portion, and then a DF image is formed with the signal corresponding to the selected reflection. Under this operation, the grains having the same orientation as the selected diffraction signal will be illuminated in the thus formed DF image, while the other region would be dark. This technique allows 
the assessment of the fraction of the transformed grains; this method will be applied in the Chapter 4 for Fe-Pt.

In HRTEM mode, a large-diameter objective diaphgram is used to allow the transmitted beam and at least one diffracted beam for imaging. All the passed beams are then made to recombine to form the image, preserving their amplitudes and phases. Spatial resolution of $0.2 \mathrm{~nm}$ has been developed recently with the advent of high-voltage commercial TEMs.

A JEOL 2000 and a HRTEM, Titan 80-300 are used for TEM analysis in this dissertation.

In TEM experiments with thin film samples, the sample preparation usually involves a two-step process: mechanical polishing and ion milling. Mechanical polishing is achieved either by using a grinder (Gatan Disc Grinder 623) to carefully polish the sample from the backside to until 50-80 $\mu \mathrm{m}$, or using a dimpling grinder (Model 200 Dimpling Grinder, Fischione Instruments) to directly dimple the sample to $\sim 20 \mu \mathrm{m}$; the grounded sample then is transferred to an ion milling instrument (Gatan Precision Ion Polishing Model 691) to obtain a hole, of which the edge is thin enough to allow the transparency of the electron beam. Since we use several different substrates for different alloy systems in this dissertation, the preparation process will be specified in the corresponding chapters.

\subsubsection{Magnetic properties}

For the Fe-Pt system, assessment of magnetic property is one of the objectives of this study. The characterization of the magnetic properties is performed using a vibrating sample magnetometer (VSM). The working principle lies in the fact that a voltage change will be generated if the magnetic flux enclosed within the pick-up coil is changed during the measurement, usually during predetermined scanning of the 
applied magnetic field. The detected signal is the magnetization of the sample as a function of the applied magnetic field. A schematic of the configuration of a typical VSM is shown in Fig. 2.4.

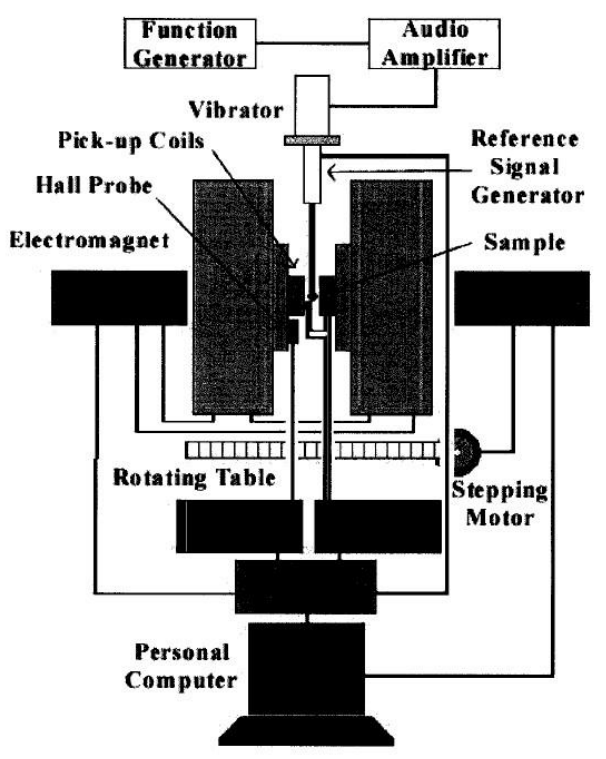

Figure 2.4 A schematic of a typical VSM [7].

In order to study the magnetic interaction, e.g., between different grains within the film, or different layers in the multilayer structures, the so-called $\delta \mathrm{M}$ method $[8,9]$ is often used. In this technique, two curves, the de demagnetization curve $I_{d}$ (DCD) and the isothermal remanent magnetization $I_{r}$ (IRM) curve are measured. In the DCD curve, the sample is first magnetized with a negative saturation field $-H_{\text {sat }}$, then a small external field is applied and then removed to obtain the remanent magnetization signal; the external field is increased with preset steps and then removed to get the corresponding remanence. This procedure is repeated up to the saturation field $H_{\text {sat }}$ to get the $I_{d}\left(H_{\text {sat }}\right)$. The measurement of $I_{r}(\operatorname{IRM})$ curve is similar to that of $I_{d}(H)$, except that the sample is first ac demagnetized instead of negatively saturated. Wohlfarth [10] proposed a relation between $I_{r}$ and $I_{d}$ for an assembly the noninteracting single domain particles with uniaxial anisotropy regardless of orientation of easy axis: 


$$
I_{d}(H)=I_{r}\left(H_{s a t}\right)-2 I_{r}(H)
$$

Henkel [11] proposed deviation from this ideal behavior due to the interaction between particles; the so-called $\delta M$ then is obtained with the following expression:

$$
\delta M(H)=I_{d}(H)-\left[I_{r}\left(H_{\text {sat }}\right)-2 I_{r}(H)\right]
$$

According to experiments and simulations and by including the grain orientation effects, generally speaking, a negative peak in the $\delta M(H)$ curve is observed for purely magnetostatic integrations, e.g., without exchange coupling; on the contrary a positive peak around the coercive field $H_{c}$ with negative $\delta M(H)$ at larger field indicates the presence of exchange interaction among nearby grains.

\subsection{Substrates}

As will be seen later in the experimental section for each alloy system, various substrates were used. For $\mathrm{CV}$ and EQCM measurement, they were performed on $\mathrm{Au}$ coated AT-cut quartz resonator, which is supplied with the EQCM system we used.

Deposition was mainly performed on Ru substrate; the $100 \mathrm{~nm}$ thick unoriented Ru layers was sputter deposited onto a $5 \mathrm{~nm}$ Ta adhesion layer on top of the native oxide of $\mathrm{Si} \mathrm{(001).} \mathrm{The} \mathrm{use} \mathrm{of} \mathrm{Ru} \mathrm{is} \mathrm{mainly} \mathrm{due} \mathrm{to} \mathrm{the} \mathrm{fact} \mathrm{that} \mathrm{it} \mathrm{is} \mathrm{stable} \mathrm{in} \mathrm{the} \mathrm{electrolyte}$ we used at open circuit potential and does not dissolve into the electrolyte without applying quite positive potentials, thus eliminating the possible contamination due to its dissolution into the electrolyte. Furthermore, $\mathrm{Ru}$ serves as good barrier for interdiffusion when the alloys are under annealing, for example, in the Fe-Pt system.

Other substrates, such as $\mathrm{Au}$ and $\mathrm{Cu}$, were also used for deposition, for which the details will be provided in corresponding chapters. The purpose was to reduce the interference of the substrate peaks with the peaks of the deposited alloys in XRD experiments due to overlapping. 


\section{References}

[1] Model QCM 922 Quartz Crystal Microbalance, Princeton Applied Research, 2009.

[2] D.A. Buttry, M.D. Ward, Chem. Rev. 92 (1992) 1355.

[3] C.R. Brundle, C.A. Evans, S. Wilson, Encyclopedia of Materials Characterization: Surfaces, Interfaces, Thin Films, Butterworth-Heinemann, Stoneham, MA, 1992.

[4] S. Zhang, L. Li, K. Ashok, Materials Characterization Techniques, CRC Press, Talor\&Francis Group, Boca Raton, FL, 2009.

[5] D.T. Carpenter, J.M. Rickman, K. Barmak, J. Appl. Phys. 84 (1998) 5843.

[6] K. Barmak, R.A. Ristau, K.R. Coffey, M.A. Parker, J.K. Howard, J. Appl. Phys. 79 (1996) 5330.

[7] Cnsm.kaist.ac, Kr/vsm.html (n.d.).

[8] F. Casoli, F. Albertini, L. Nasi, S. Fabbrici, R. Cabassi, F. Bolzoni, C. Bocchi, Appl. Phys. Lett. 92 (2008) 142506.

[9] L. Callegaro, E. Puppin, P.L. Cavallotti, G. Zangari, J. Magn. Magn. Mater. 155 (1996) 190.

[10] E.P. Wohlfarth, J. Appl. Phys. 29 (1958) 595.

[11] O. Henkel, Phys. Status Solidi B 15 (1966) 211. 


\section{Chapter 3 Electrodeposition of Cu-Au alloys}

\section{Overview}

$\mathrm{Au}-\mathrm{Cu}$ based alloys are interesting in electronic industry for applications, e.g., as contacts, due to their excellent conductivity and resistance to corrosion and reduced price compared with pure $\mathrm{Au}$ parts [1]. From the thermodynamic standpoint, $\mathrm{Au}-\mathrm{Cu}$ is miscible for the whole composition range at room temperature, while there exist three ordered structures, $\mathrm{L1}_{2} \quad \mathrm{Au}_{3} \mathrm{Cu}, \quad \mathrm{L1}_{0} \quad \mathrm{AuCu}$ and $\mathrm{L1}_{2} \mathrm{AuCu}_{3}$ [2,3]. In electrochemistry, $\mathrm{Au}-\mathrm{Cu}$ is a prototypical system, especially due to the so called $\mathrm{Cu}$ underpotenial deposition (UPD) on $\mathrm{Au}[4,5]$. Another advantage for electrochemical study of $\mathrm{Au}-\mathrm{Cu}$ system lies in the fact that both of the two constituents can be deposited at potentials more positive than HER, thus excluding this parasitic reaction effect.

$\mathrm{Au}-\mathrm{Cu}$ alloy deposition from weakly complexed solution was first studied; as will be seen, deposits from this solution are porous and rough, yielding powder structures [6,7]. To address this issue, we also studied the UPCD behavior of Au-Cu alloys from a strong complexed solution for both $\mathrm{Au}$ and $\mathrm{Cu}$; we found that very uniform composition distribution is obtained in the deposits and films are very smooth and shiny. Finally, we will show an anomalous UPCD behavior, that is, by complexing $\mathrm{Au}$ and $\mathrm{Cu}$ selectively, we are able to make $\mathrm{Cu}$ more noble than $\mathrm{Au}$, leading to $\mathrm{Au}$ UPCD with $\mathrm{Cu}$, which is named as reverse UPCD.

\subsection{Experimental}

For the weakly complexed solution, sulfate based electrolytes were used, with various concentrations of $\mathrm{HAuCl}_{4}$ and $\mathrm{CuSO}_{4}$, while $0.5 \mathrm{M} \mathrm{H}_{2} \mathrm{SO}_{4}$ was added as supporting electrolyte; natural $\mathrm{pH}$ was used, which was $\sim 0.5-0.8$ 
For the complexed solution of $\mathrm{Cu}-\mathrm{Au}$ deposition, an ethylenedamine (EN) compound, $\mathrm{Au}(\mathrm{EN})_{2}$, was synthesized following literature [8]. The final solution contains $2 \mathrm{mM}$ $\mathrm{Au}(\mathrm{EN})_{2}, 20 \mathrm{mM} \mathrm{CuSO}_{4}, 0.2 \mathrm{M}$ glycine and $0.2 \mathrm{M} \mathrm{Na}_{2} \mathrm{SO}_{4}$ as supporting electrolyte; $\mathrm{pH}$ was set as 7.0. The details of the electrolyte will be discussed in the solution chemistry in the following.

For the reverse UPCD, homemade sulfite based electrolytes were used. The stability of this homemade solution is short; it lasts about two days and then precipitates out some colloids, which is probably due to the disproportionation reaction [9] of:

$$
2 A u^{+} \rightarrow A u+A u^{3+}
$$

Commercial gold sulfite solutions usually contain stabilizing additives to suppress this reaction to obtain longer duration. We however used the homemade one here to avoid the interference of unknown additives, in order to show the reverse UPCD process; solutions were made right before usage and experiments were completed within one day before any visible deterioration of the solution. Thus, the stability issue does not impair the investigation in UPCD behavior.

For comparison with $\mathrm{Cu}$ UPCD with $\mathrm{Au}$, the metal concentrations of $\mathrm{Cu}$ and $\mathrm{Au}$ in the sulfite solution are $2 \mathrm{mM} \mathrm{CuSO}_{4}$ and $20 \mathrm{mM} \mathrm{HAuCl}_{4}$, respectively. Large excess of $0.168 \mathrm{M} \mathrm{Na}_{2} \mathrm{SO}_{3}$ was used as complexing agent, so that both $\mathrm{Au}$ and $\mathrm{Cu}$ can fully develop their own complexes regardless of the other. Upon mixing with sulfite, $\mathrm{Au}^{3+}$ is reduced to form $\mathrm{Au}^{+}$complexes [10,11], while $\mathrm{Cu}^{2+}$ is reduced to form corresponding $\mathrm{Cu}^{+}$complexes [12], the strength of both depending on $\mathrm{pH}$. We here set the $\mathrm{pH}$ at 8.0 since the sulfite solution is more stable at alkaline $\mathrm{pHs}$.

The electrochemical behavior for all these solution was investigated with electrochemical quartz crystal microbalance (EQCM) [5,13-15]. EQCM and corresponding CVs were carried out with $\mathrm{Au}$ (111) resonator with a scan rate of $5 \mathrm{mV} / \mathrm{s}$ without deaeration or stirring during measurement; solutions were however 
deaerated for $1 \mathrm{~h}$ before experiments by purging nitrogen gas. The $100 \mathrm{~nm}$ thick unoriented $\mathrm{Ru}$ layers, sputter deposited onto a $5 \mathrm{~nm}$ Ta adhesion layer on top of the native oxide of $\mathrm{Si}(001)$ wafers were used as substrates for alloy deposition. $\mathrm{Au}-\mathrm{Cu}$ films were electrodeposited under constant potential on $\mathrm{Ru}$. Film growth experiments were performed using a three-electrode cylinder cell in the vertical configuration. The counter-electrode was a Pt mesh, and the reference was a saturated mercury sulfate electrode (MSE). In the following all potentials will be referred to MSE ( $E_{M S E}^{0}=0.650$ $\left.\mathrm{V}_{\text {SHE}}\right)$.

Alloy composition was determined by energy dispersive spectroscopy EDS using a detector attached to a JEOL JSM-6700F scanning electron microscope (SEM). The ratio $\mathrm{Cu}$ to $\mathrm{Au}$ content was determined using an accelerating voltage of $20 \mathrm{kV}$ and the $\mathrm{K}$ line for $\mathrm{Cu}$, the $\mathrm{M}$ line for $\mathrm{Au}$. The suifite solution was observed to yield $\mathrm{S}$ impurities in the alloy; S content was evaluated using the $\mathrm{K}$ line. Morphology was obtained with the above SEM. Crystal structure was determined by X-ray diffraction, using a Panalytical X'Pert diffractomer with $\mathrm{Cu} \mathrm{K} \alpha$ radiation $(\lambda=1.5418 \AA)$ in the $\theta / 2 \theta$ geometry.

\subsection{Electrodeposition of $\mathrm{Au}-\mathrm{Cu}$ alloys from weakly complexed solutions}

In Fig.3.1, the EQCM data for $0.5 \mathrm{mM} \mathrm{HAulCl}_{4}+0.05 \mathrm{M} \mathrm{CuSO}_{4}+0.5 \mathrm{M} \mathrm{H}_{2} \mathrm{SO}_{4}$ are shown. The deposition rate, $\mathrm{dm} / \mathrm{dt}$ by taking derivative of the mass with time, is more or less unchanged until $\sim-0.22 \mathrm{~V}$; this corresponds to the diffusion limited deposition of $\mathrm{Au}$ from the weakly complexed $\mathrm{HAulCl}_{4}$; deposition rate is about $28 \mathrm{ng} / \mathrm{cm}^{2} / \mathrm{s}$. When potentials are more negative than $-0.22 \mathrm{~V}$, a gradual increase in deposition rate is observed until a dramatic increase occurs at $\sim 0.405 \mathrm{~V}$. The drastic increase in the latter is attributed to onset of bulk $\mathrm{Cu}$ deposition. The slow increase in deposition between $-0.22 \mathrm{~V}$ and bulk deposition is due to $\mathrm{Cu}$ UPCD with $\mathrm{Au}$, with formation of 
$\mathrm{Au}-\mathrm{Cu}$ solid solutions, which is confirmed both by composition at these potentials and by crystal structure determination of films deposited in this potential range. The depolarization of $\mathrm{Cu}$ due to UPCD with $\mathrm{Au}$ is $\sim 225 \mathrm{mVs}$. In the reverse scan, bulk deposited $\mathrm{Cu}$ is stripped first, indicated by the large and more negative anodic peak at $-0.35 \mathrm{~V}$; the smaller peak at $-0.15 \mathrm{~V}$ is attributed to the stripping of alloyed $\mathrm{Cu}$, stripping of which is inhibited due to both alloying with Au and percolation through the deposited alloy.

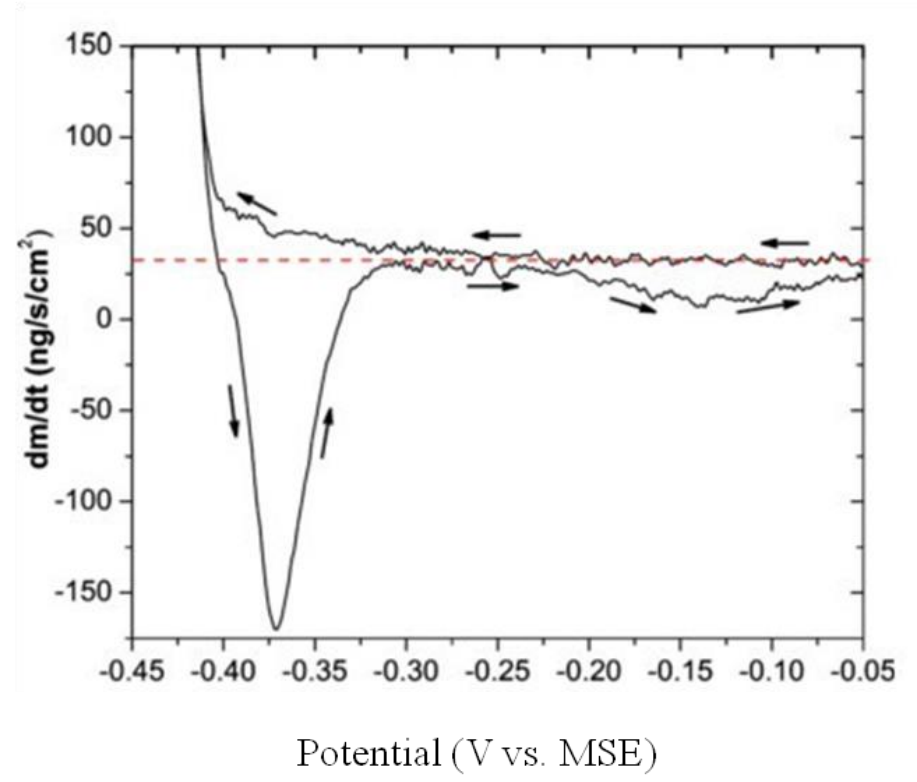

Figure 3.1 EQCM data for $0.5 \mathrm{mM} \mathrm{HAulCl}_{4}+0.05 \mathrm{M} \mathrm{CuSO}_{4}+0.5 \mathrm{M} \mathrm{H}_{2} \mathrm{SO}_{4}$; scan rate of $2 \mathrm{mV} / \mathrm{s}$ on $\mathrm{Au}[6]$.

In Fig.3.2, a set of XRD patterns for films deposited from $1 \mathrm{mM} \mathrm{Au}+0.3 \mathrm{M} \mathrm{Cu}$ is shown. With potential going more negative, and therefore increasing $\mathrm{Cu}$ content, both the (111) and (200) peaks shift to higher angles, consistent with the formation of a series of continuous FCC solid solutions, with decrease of lattice parameter. Precipitation of $\mathrm{Cu}$ was observed for $\mathrm{Cu}$ rich alloy deposited at $-0.405 \mathrm{~V}$. By fitting the (111) diffraction peak shown in Fig.3.2, the lattice parameter can be extracted and compared with known lattice parameters as a function of $\mathrm{Cu}$ content, thus obtaining alloy composition; this is shown in Fig.3.3. 


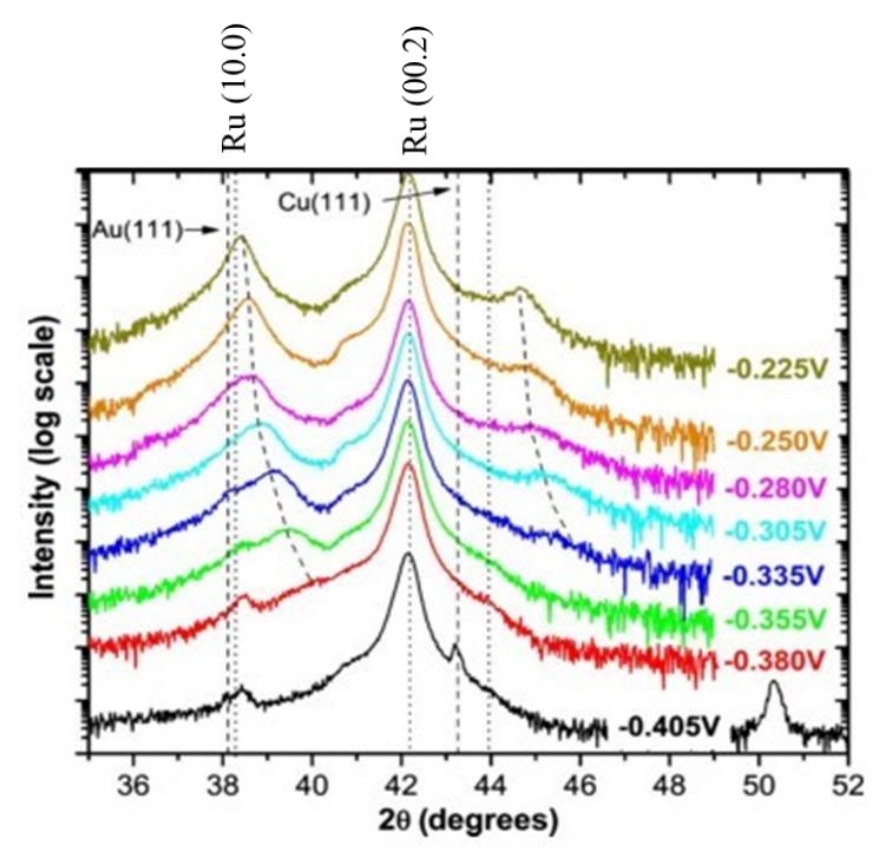

Figure 3.2 XRD patterns for films deposited from $1 \mathrm{mM} \mathrm{HAuCl}{ }_{4}+0.3 \mathrm{M} \mathrm{CuSO}_{4}$ on $\mathrm{Ru}$ [6].

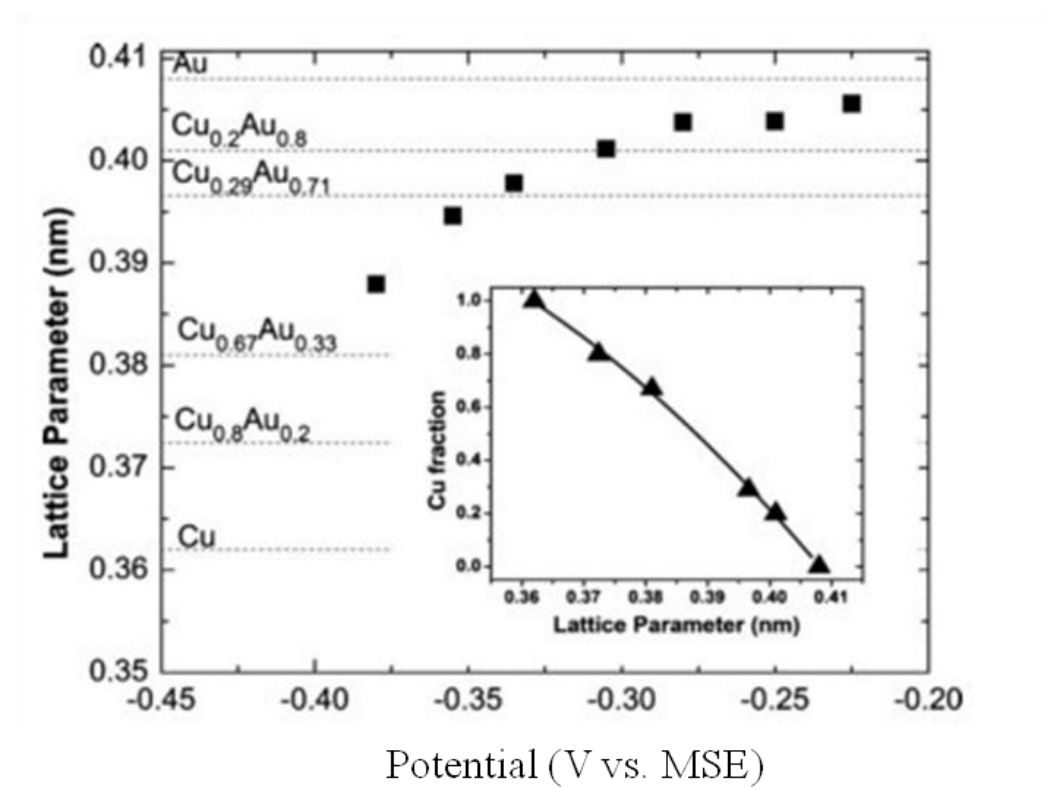

Figure 3.3 Lattice parameter as a function of deposition potential by fitting the (111) peak in Fig.3.2 [6]. The dash lines correspond to known lattice parameters for bulk alloys. The inset shows the lattice parameter as a function of $\mathrm{Cu}$ content for bulk alloy [16].

Data for composition as a function of potential for $\mathrm{Au}-\mathrm{Cu}$ alloys (nominal $300 \mathrm{~nm}$ thick) are displayed in Fig.3.4, with data determined by EDS and XRD for some films as well. There is some $20 \mathrm{mV}$ shift in the horizontal direction between $0.3 \mathrm{M}$ and $0.05 \mathrm{M} \mathrm{Cu}$, which is due to the more negative Nernst potential for smaller $\mathrm{Cu}$ concentration, $23 \mathrm{mV}$ by calculation. The lines are fittings to the experimental data 
using the sub-regular solution model shown in Chapter 1, yielding two Margules parameters and the redox potential for $\mathrm{Cu}$ deposition; the redox potentials agree well with the measurement of open circuit potentials with corresponding $\mathrm{Cu}$ concentrations in the solution without Au compound.

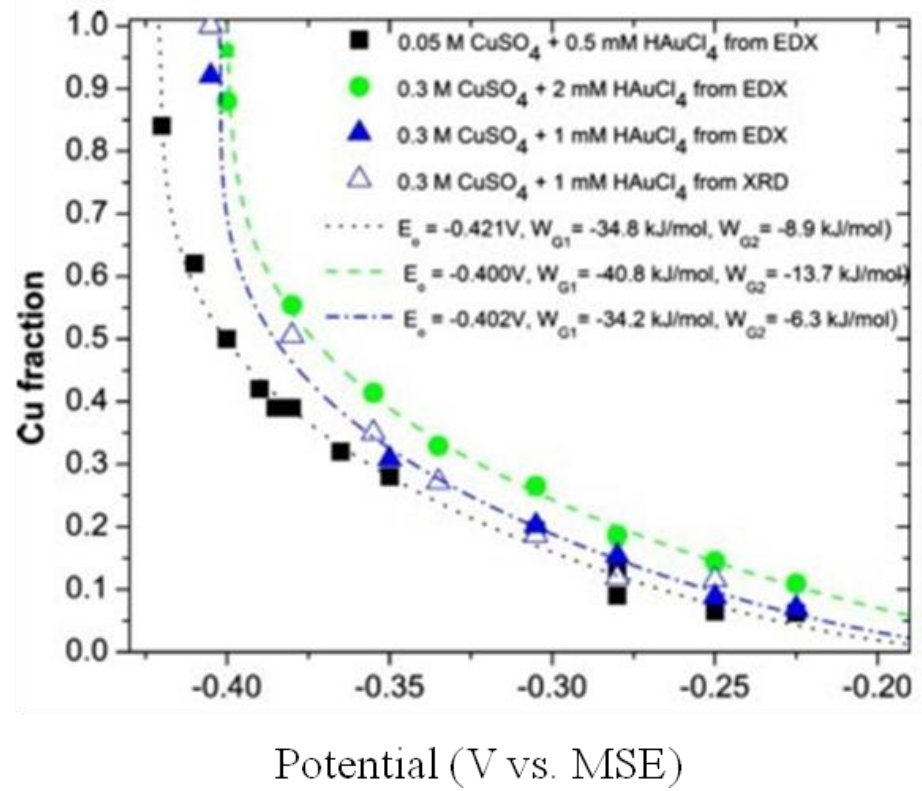

Figure 3.4 Composition vs. potential for $\mathrm{Au}-\mathrm{Cu}$ alloys deposited from solution with various $\mathrm{Au}$ and $\mathrm{Cu}$ concentrations [6]. The solid symbols represent data from EDS, the open triangles represent data extracted from XRD in Fig.3.2 and 3.3. The lines are fittings to the data by the sub-regular solution model discussed in Chapter 1, yielding two Margules parameters and the redox potential for $\mathrm{Cu}$ deposition.

The morphology of the alloys deposited from $1 \mathrm{mM} \mathrm{Au}+0.3 \mathrm{M} \mathrm{Cu}$ solution with various $\mathrm{Cu}$ contents are shown in Fig.3.5. Optically, they are very dark, rough and with bad adhesion to the substrates, and easily peel off especially for Au rich films.

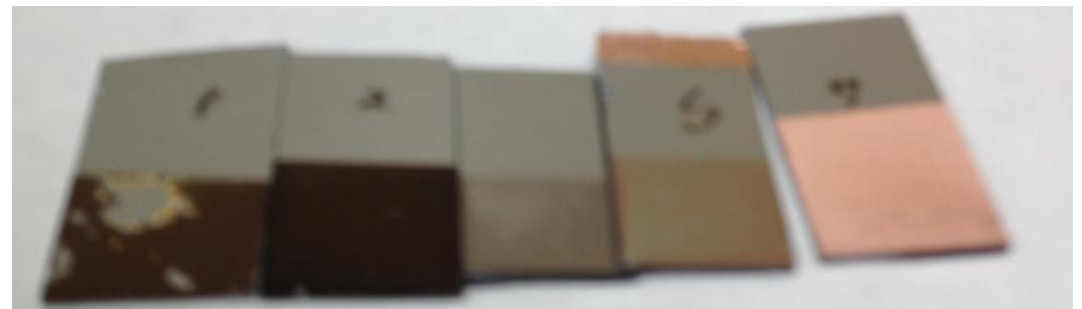

Figure 3.5 Optical images for $\mathrm{Au}-\mathrm{Cu}$ alloys from weakly complexed solution with various $\mathrm{Cu}$ contents (from left to right): 10, 20, 37, 84 and 99at. \% of $\mathrm{Cu}$. 

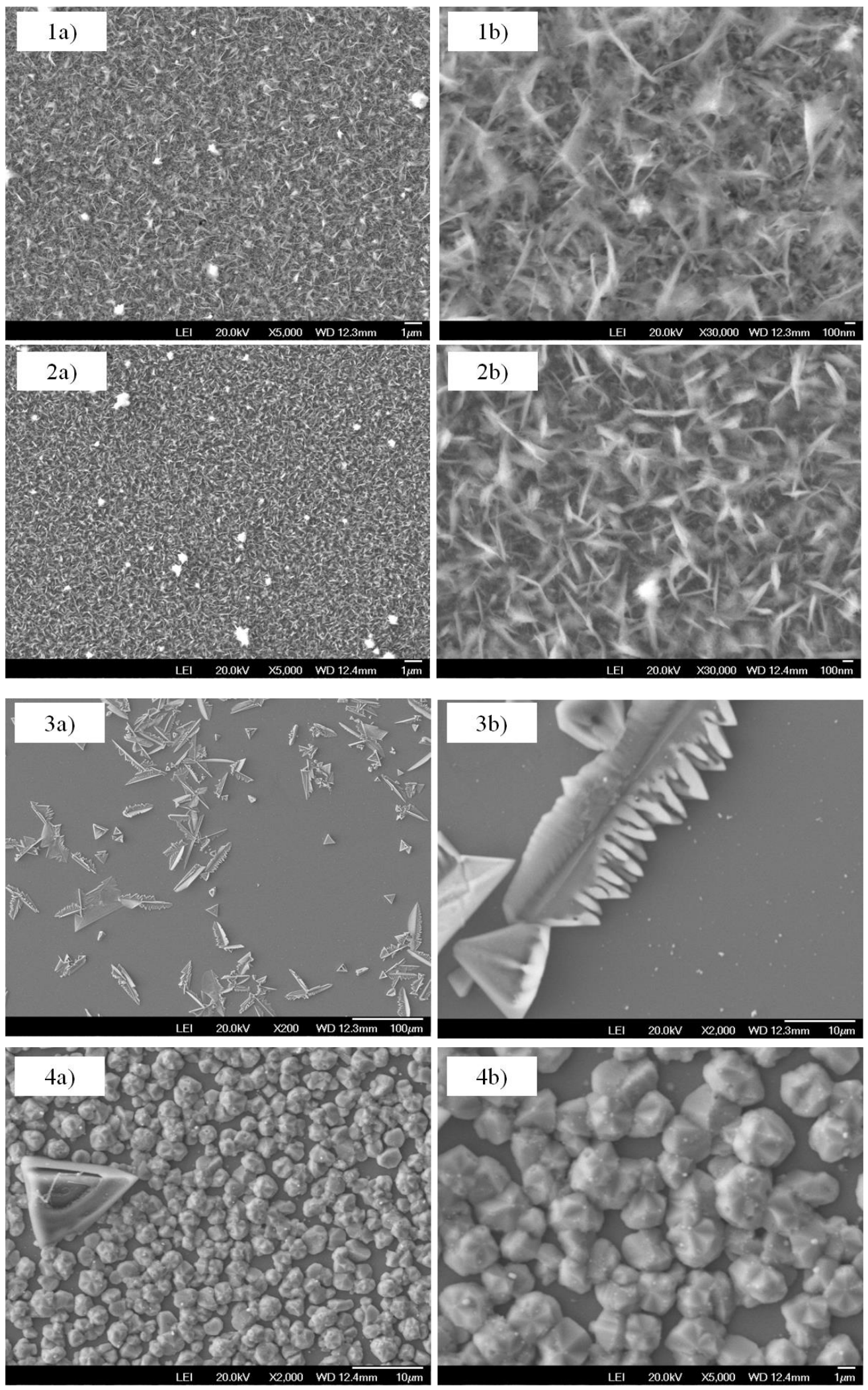

Figure 3.6 SEM images for Au-Cu alloys from weakly complexed solution with various $\mathrm{Cu}$ contents (from left to right): 1) 10;2) 20;3) 84 and 4) 99 at. \% of $\mathrm{Cu}$ (notice that to best show features of different samples, various magnifications were used accordingly); they were deposited at $-0.25 \mathrm{~V},-0.305 \mathrm{~V},-0.40 \mathrm{~V}$ and $-0.41 \mathrm{~V}$. 
SEM images for these films are shown in Fig.3.6. Au rich films show a uniform microstructure with leaf and spongy features; in the intermediate composition range, a relatively smooth smooth layer was deposited first, followed by the formation of dendrites. In $\mathrm{Cu}$-rich films, faceted grains were observed, similarly to pure $\mathrm{Cu}$ deposited from comparable solutions without Au but with chloride [17].

In order to investigate the roughening transition from a smooth layer to a rough surface (Fig. 3.6), various films were grown at two selected potentials, and composition as well as morphology were studied as a function of thickness. Shown in Fig.3.7 is composition vs. thickness for two alloys containing 20at.\% and 43at.\% of $\mathrm{Cu}$ when reaching the steady state composition. $\mathrm{Cu}$ content was observed to increase with thickness up to $\sim 40 \mathrm{~nm}$, then remaining the same upon increasing thickness.

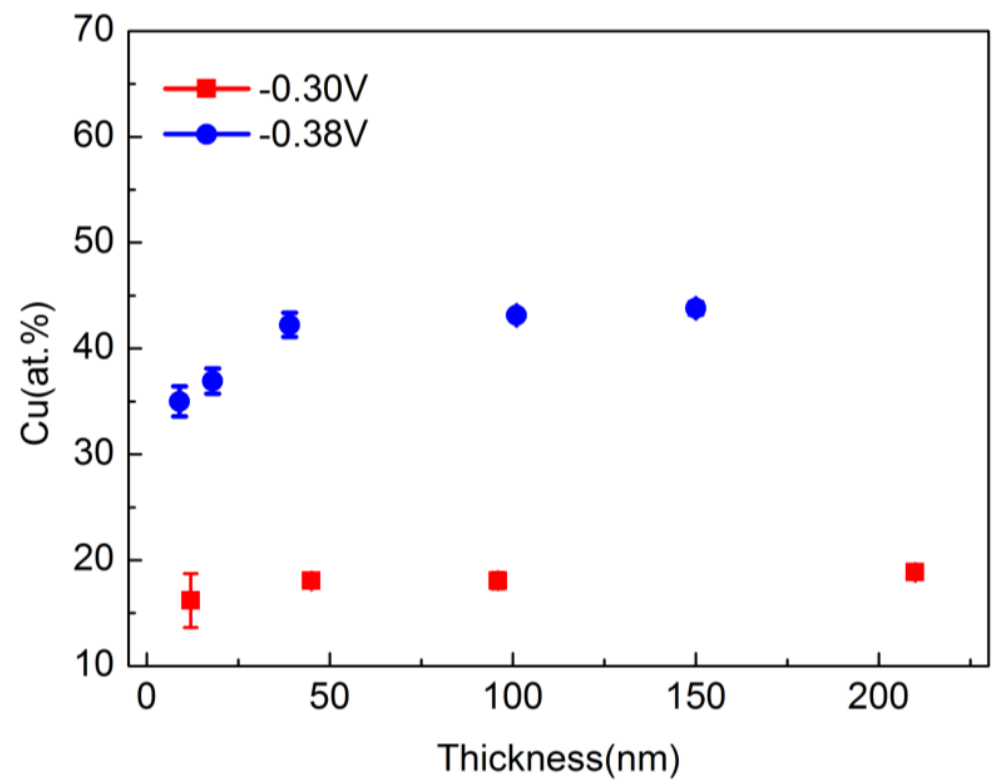

Figure 3.7 Composition as a function of thickness for alloys deposited $-0.30 \mathrm{~V}$ and $-0.38 \mathrm{~V}$, respectively. 

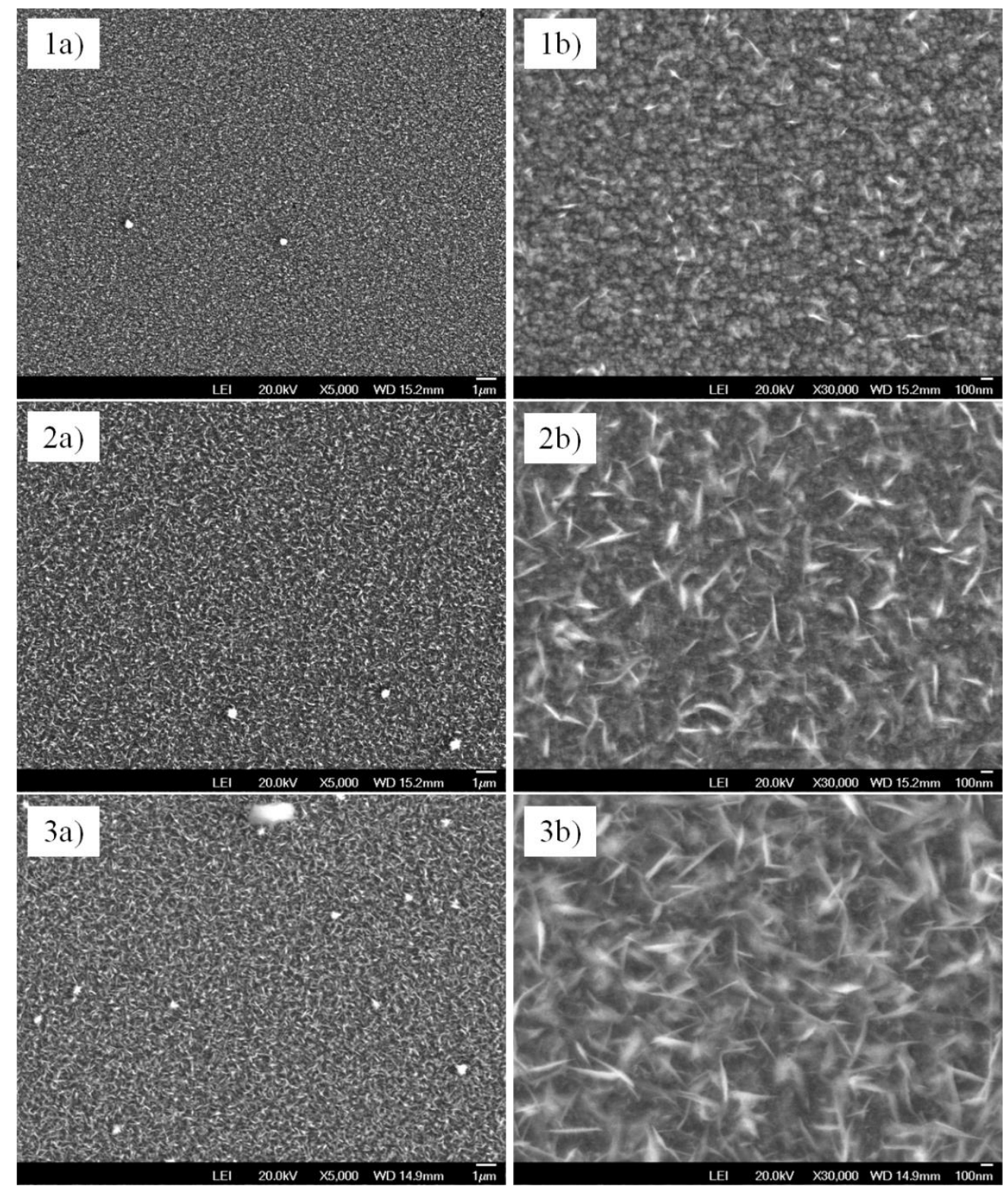

A) 


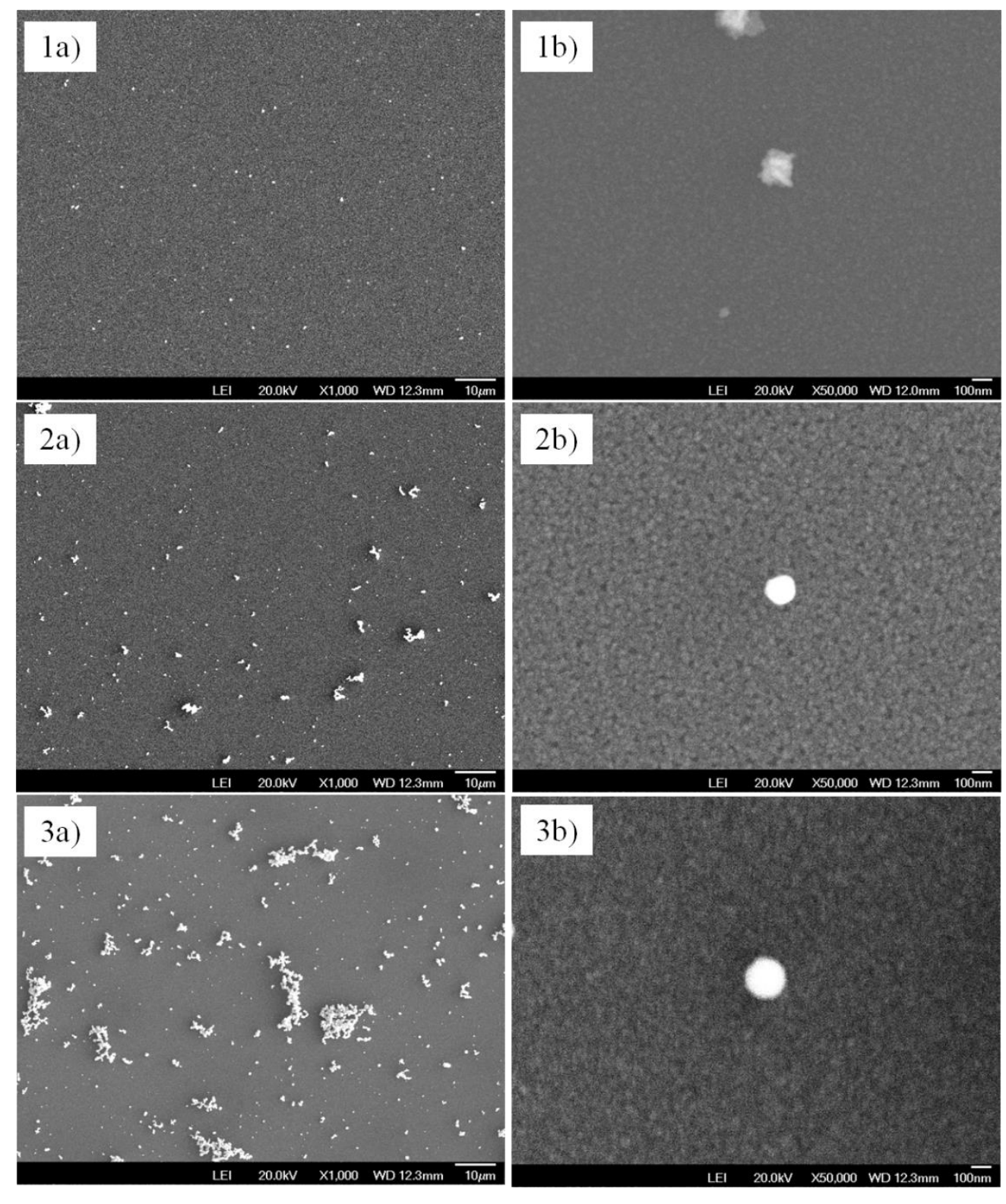

B)

Figure 3.8 SEM images and corresponding compositions of films deposited at $-0.30 \mathrm{~V}$ and $-0.38 \mathrm{~V}$ with various thickness, showing that rough surface formation after certain thickness of smooth layer: A) for $-0.30 \mathrm{~V}(1: 12 \mathrm{~nm}, 245 \mathrm{~nm}$, and $396 \mathrm{~nm}$ ); B for $-0.38 \mathrm{~V}(19 \mathrm{~nm}, 218 \mathrm{~nm}$ and 339 $\mathrm{nm})$. 


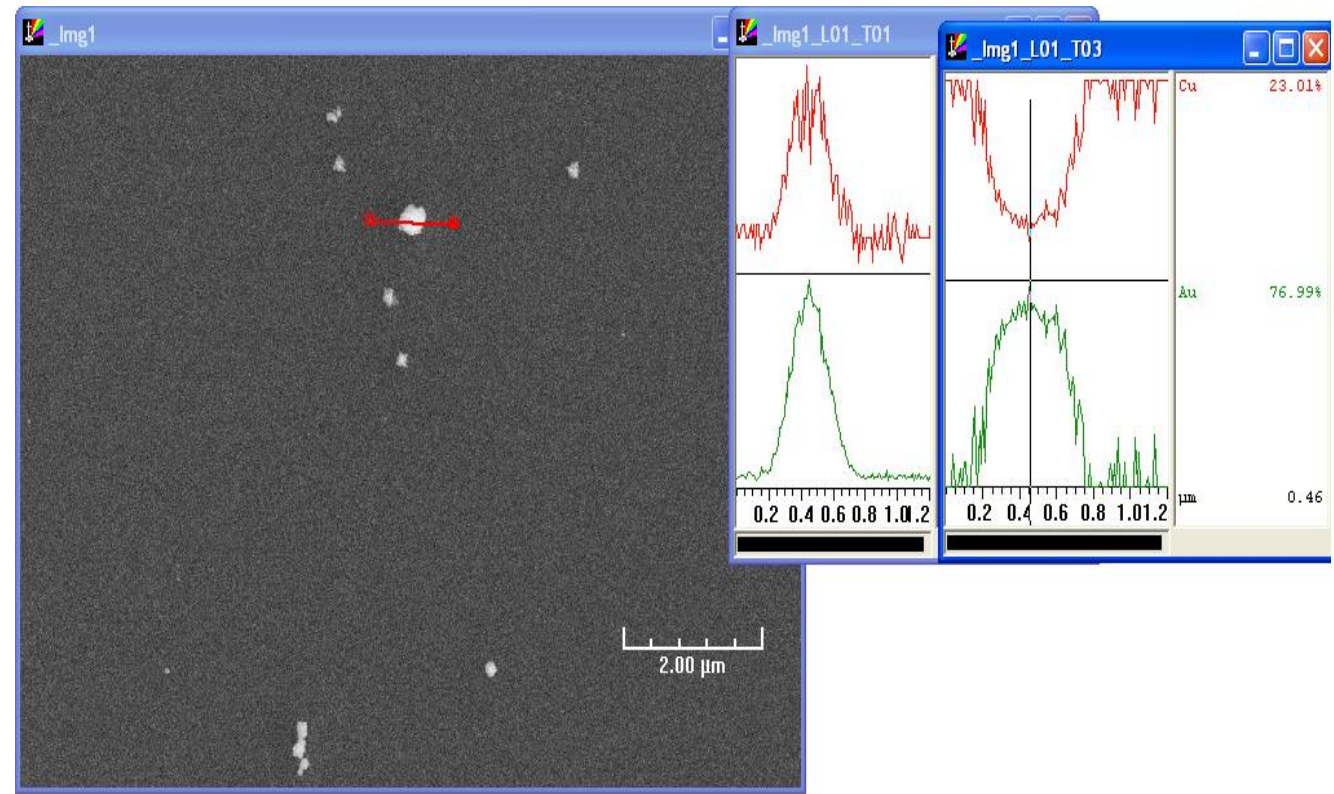

Figure 3.9 EDS line scan showing that the rough surface is richer in Au than the overall composition.

The morphology as a function of thickness for these films is shown in Fig.3.8. Films deposited at $-0.30 \mathrm{~V}$ exhibit a leaf or needle like structure already at $12 \mathrm{~nm}$, and the thicker the film, the higher the surface coverage of such a structure; for films deposited at $-0.38 \mathrm{~V}$ with higher $\mathrm{Cu}$ content, smooth layers with apparent grain size of $\sim 30 \mathrm{~nm}$ were clearly present up to $39 \mathrm{~nm}$. Clusters of particles, which are richer in Au compared with the overall composition (Fig.3.9), appear for all thicknesses; as thickness increases, the size of these clusters increase as well as the density.

\subsection{Electrodeposition of $\mathrm{Au}-\mathrm{Cu}$ alloys from $\mathrm{Au}(\mathrm{EN})_{2}$ solutions}

As it is shown above, the deposits from the weakly complexed $\mathrm{HAuCl}_{4}$ based solutions are powdery, rough and are susceptible to roughening due to growth instability, resulting from the diffusion limited deposition of $\mathrm{Au}$ when $\mathrm{Cu}$ is co-deposited. To address this issue, it is possible to polarize Au deposition so as to avoid or mitigate the diffusion limited deposition of $\mathrm{Au}$; this requires much stronger complexed Au compounds. One of such compound is Au cyanide [1]. However, due 
to its high toxicity, strong restrictions are placed on its usage. It is therefore necessary to find alternative compounds that may meet both low or non-toxicity and stability requirements. We show here that an EN based Au [8] compound is excellent in regard to these aspects.

\subsubsection{Solution chemistry}

$\mathrm{Au}(\mathrm{EN})_{2}$ is stable in neutral and alkaline $\mathrm{pHs}$ [8]. At these pHs, however, the solubility of $\mathrm{Cu}^{2+}$ is very small and precipitation of hydroxide will occur. To avoid that, glycine $[18,19]$ is used to complex $\mathrm{Cu}^{2+}$ and therefore reduce free $\mathrm{Cu}^{2+}$ under the solubility limit. To better understand the solution chemistry of $\mathrm{Cu}$ with glycine, we determined the free $\left[\mathrm{Cu}^{2+}\right]$ as a function of $\mathrm{pH}$, concentration of $\mathrm{CuSO}_{4}$ and glycine by solving a system of chemical equilibria (Table 3.1). We finally decided to use 0.5 mM- $2 \mathrm{mM} \mathrm{Au}(\mathrm{EN})_{2}, 20 \mathrm{mM} \mathrm{CuSO}{ }_{4}$ and $0.2 \mathrm{M}$ glycine. The concentration of free

$\mathrm{Cu}^{2+}$ is calculated then as a function of $\mathrm{pH}$; EN can also complex with $\mathrm{Cu}^{2+}$ if dissociated from Au compound; this possibility however is difficult to assess and is not

Table 3.1. Equilibria and stability constants

\begin{tabular}{|c|c|c|c|c|}
\hline $\begin{array}{c}\text { Complexing } \\
\text { agent }\end{array}$ & \multicolumn{2}{|c|}{ Equilibrium equations } & $\begin{array}{l}\text { Equilibrium } \\
\text { constants( } \log )\end{array}$ & $\begin{array}{c}\text { Equation } \\
\text { No. }\end{array}$ \\
\hline \multirow{5}{*}{$\begin{array}{c}\mathrm{NH}_{2} \mathrm{CH}_{2} \mathrm{COO} \\
\mathrm{H} \\
\text { (short for HL) }\end{array}$} & & $H^{+}+L^{-} \Leftrightarrow H L$ & $K=9.7$ & $1[18]$ \\
\hline & Deionization & $H L+H^{+} \Leftrightarrow H_{2} L^{+}$ & $K=2.46$ & $2[18]$ \\
\hline & & $C u^{2+}+L^{-} \Leftrightarrow[C u L]^{+}$ & $K_{1}=8.11$ & $3[18]$ \\
\hline & $\begin{array}{l}\text { Association } \\
\text { with } \mathrm{Cu}^{2+}\end{array}$ & $C u^{2+}+2 L \Leftrightarrow\left[C u L_{2}\right]$ & $\beta_{2}=14.43$ & $4[18]$ \\
\hline & & {$[\mathrm{CuL}]^{+}+H^{+} \Leftrightarrow[\mathrm{CuHL}]^{2+}$} & $\beta=2.92$ & $5[19]$ \\
\hline
\end{tabular}




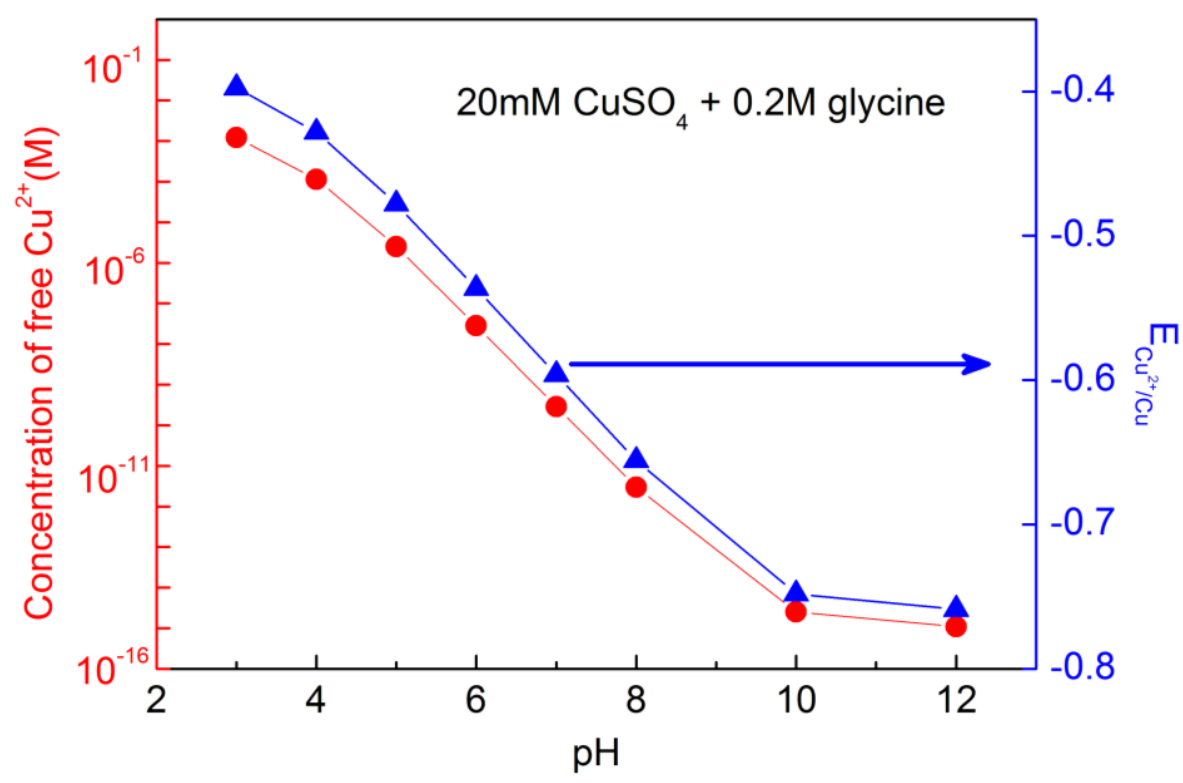

Figure 3.10 Concentration of free $\mathrm{Cu}^{2+}$ as a function of $\mathrm{pH}$ with set $20 \mathrm{mM} \mathrm{CuSO}_{4}+0.2 \mathrm{M}$ glycine.

taken into account here due to its low concentration compared with the large excess of glycine, and the $\operatorname{Au}(\mathrm{EN})_{2}$ is formed and difficult to dissociate. The data thus obtained are shown in Fig.3.10. Neutral pH was used for this deposition process; the redox potential for $\mathrm{Cu}$ is observed to shift from $-0.37 \mathrm{~V}$ calculated from the Nernst equation to $-0.59 \mathrm{~V}$ at $\mathrm{pH}$ 7.0. In the following, $0.2 \mathrm{M}$ glycine $+0.2 \mathrm{M} \mathrm{Na}_{2} \mathrm{SO}_{4}$ without either $\mathrm{Au}$ or $\mathrm{Cu}$ is denoted as background solution.

\subsubsection{Electrochemical characterization}

The electrochemical behavior during deposition of $\mathrm{Au}-\mathrm{Cu}$ from $\mathrm{EN}$ based solution was characterized by EQCM. In Fig.3.11 a), the CV and dm/dt data for only $2 \mathrm{mM}$ $\mathrm{Au}(\mathrm{EN})_{2}$ with background solution are shown. Au deposition starts at $\sim-0.18 \mathrm{~V}$, compared with $0.30 \mathrm{~V}$ in the weakly complexed case, leading to a polarization of $480 \mathrm{mV}$. Deposition of $\mathrm{Au}$ reaches a peak at $-0.59 \mathrm{~V}$ and the plateau signifying diffusion limited deposition starts from $-0.75 \mathrm{~V}$. In the reverse scan, no dissolution of $\mathrm{Au}$ is observed up to $0 \mathrm{~V}$. The current efficiency is well above $67 \%$ for the whole potential range. 

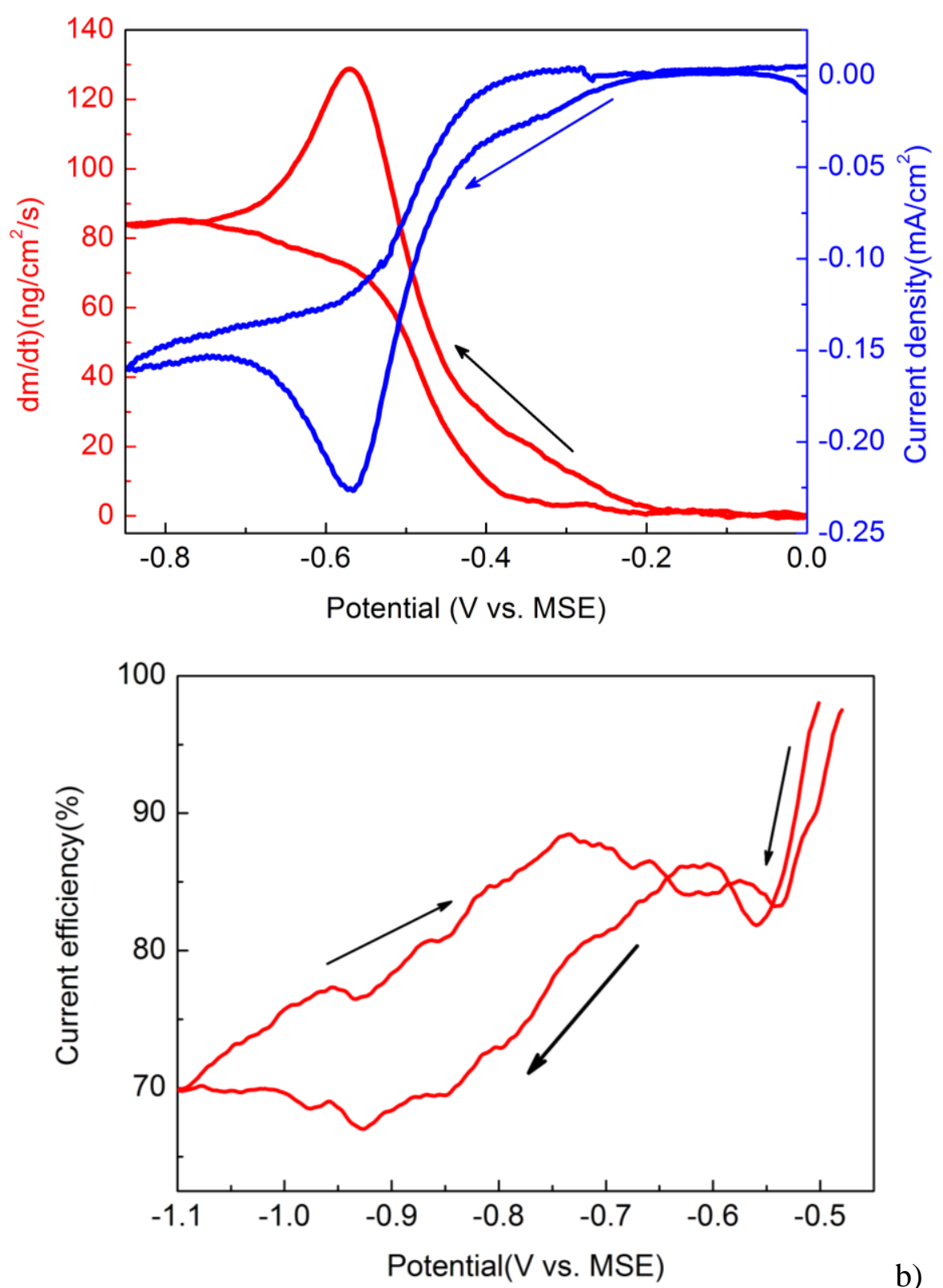

a)

b)

Figure 3.11 a) $\mathrm{CV}$ and dm/dt data; b) current efficiency for $2 \mathrm{mM} \mathrm{Au}(\mathrm{EN})_{2}+$ background solution on $\mathrm{Au}$ resonator, $\mathrm{pH}$ 7.0. Scan rate: $5 \mathrm{mV} / \mathrm{s}$. 


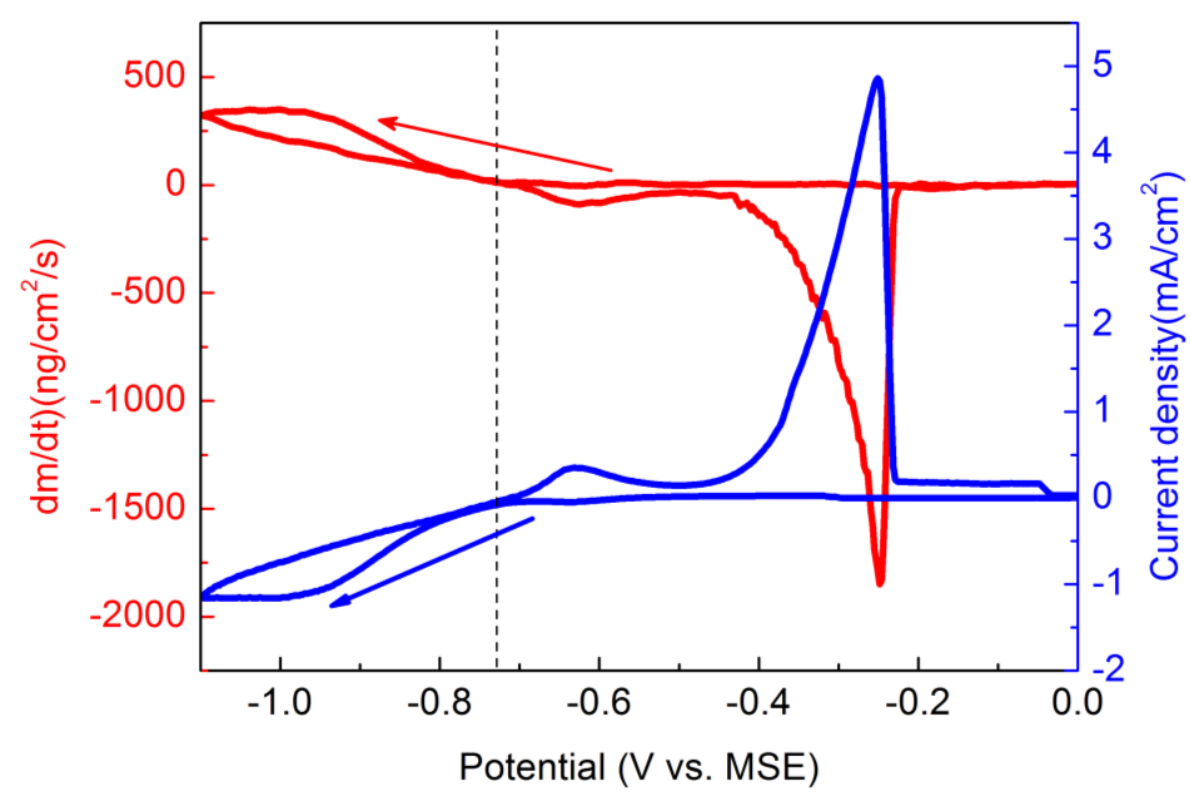

a)

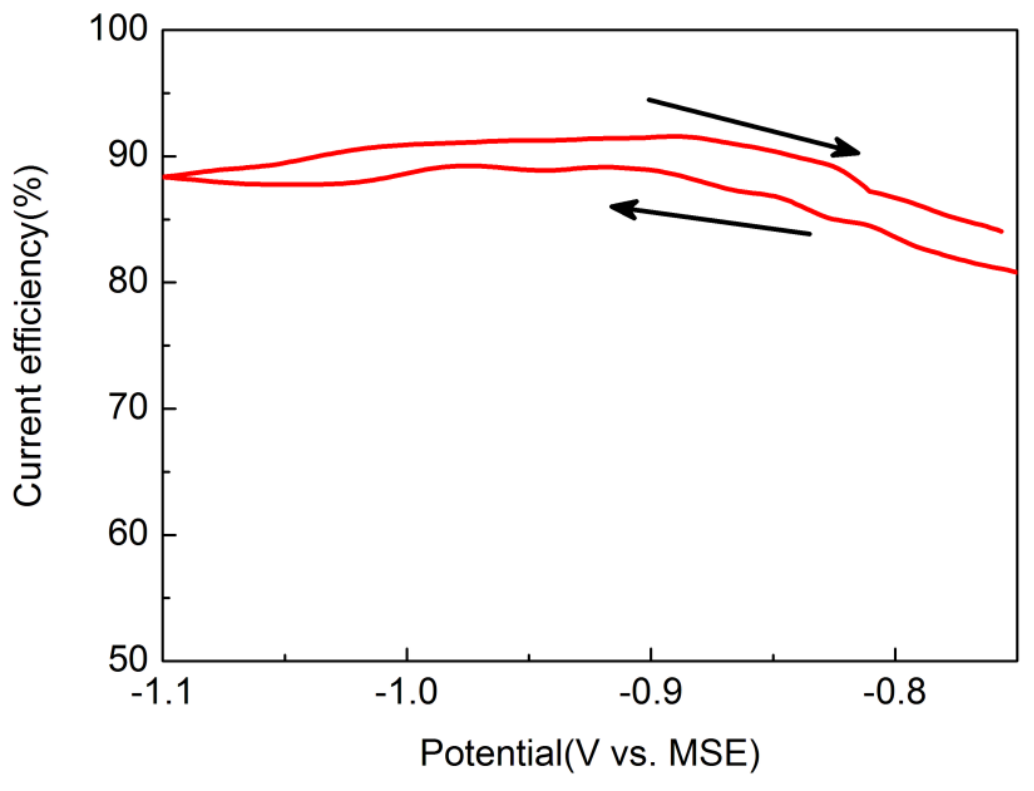

b)

Figure 3.12 a) $\mathrm{CV}$ and dm/dt data; and b) current efficiency for $20 \mathrm{mM} \mathrm{CuSO}_{4}+$ background solution on Au resonator, $\mathrm{pH}$ 7.0. Scan rate: $5 \mathrm{mV} / \mathrm{s}$.

Shown in Fig.3.12 are the $\mathrm{CV}$ and dm/dt data for only $20 \mathrm{mM} \mathrm{Cu}$ with the background solution. $\mathrm{Cu}$ deposition starts at $\sim-0.73 \mathrm{~V}$, about $100 \mathrm{mV}$ more negative than the value calculated from chemical equilibrium calculation. This may be due to the fact that chemical stability constants are not very accurate and additional complexes such as multiple complexes may exist which haven't been considered . It should be noticed, however, that the onset of $\mathrm{Cu}$ deposition is more positive than that of $\mathrm{Au}$ diffusion deposition at $-0.75 \mathrm{~V}$. If UPCD occurs in these complexing 
electrolytes with a shift of $\sim 225 \mathrm{mV}$ as in the weakly complexed solution shown above, we should be able to avoid diffusion limited deposition of $\mathrm{Au}$ when $\mathrm{Cu}$ is co-deposited; this will be checked in the following. Current efficiency is well above 80\%, as shown in Fig.3.12 b).

The $\mathrm{CV}$ and $\mathrm{dm} / \mathrm{dt}$ data for the solution combining both $\mathrm{Au}$ and $\mathrm{Cu}$ are shown in Fig.3.13. The diffusion limited deposition peak for $\mathrm{Au}$ is no longer evident; gradual increase in deposition rate before bulk $\mathrm{Cu}$ deposition is observed between $-0.55 \mathrm{~V}$ to $-0.71 \mathrm{~V}$. To have a better idea of the electrochemical behaviors for the solution shown above with $\mathrm{Au}, \mathrm{Cu}$ and $\mathrm{Au}+\mathrm{Cu}$, the dm/dt data are plotted together and shown in Fig.3. 14. Due to the not evident

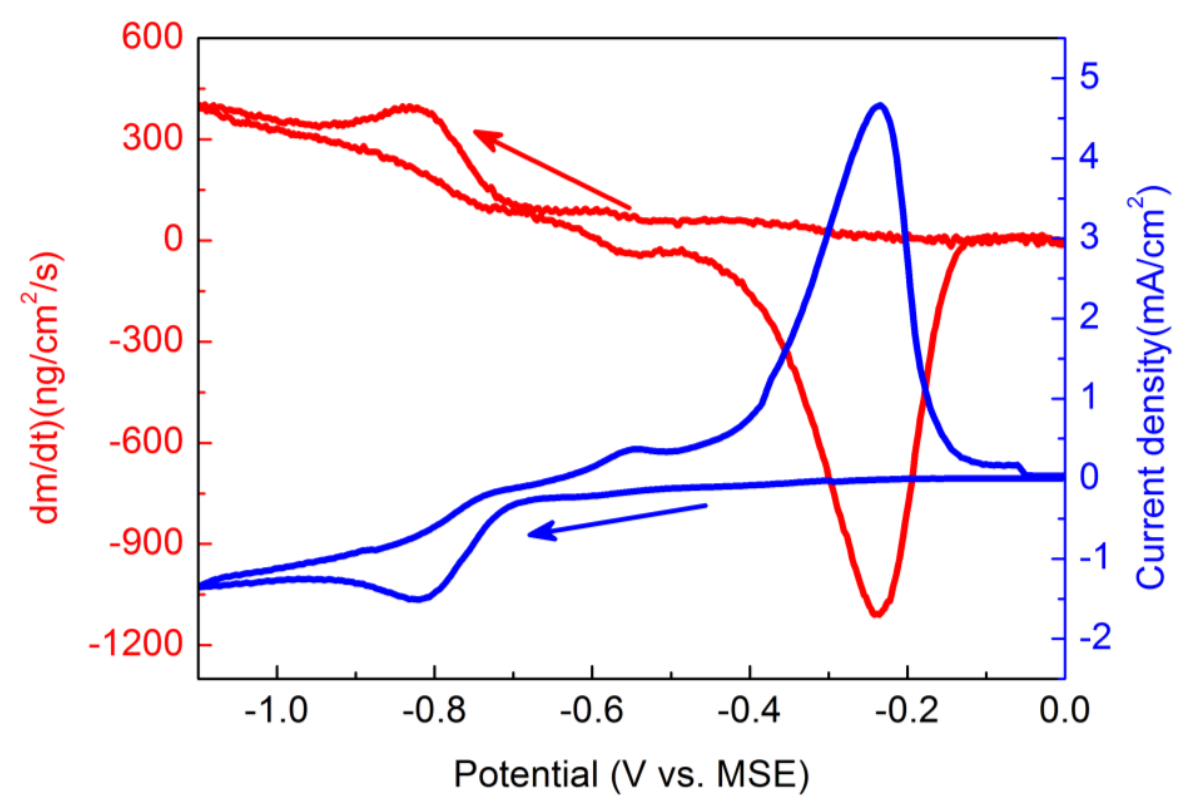

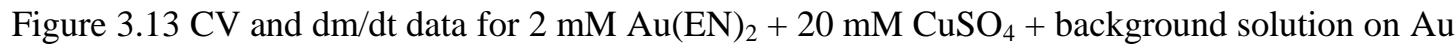
resonator, $\mathrm{pH}$ 7.0. Scan rate: $5 \mathrm{mV} / \mathrm{s}$. 


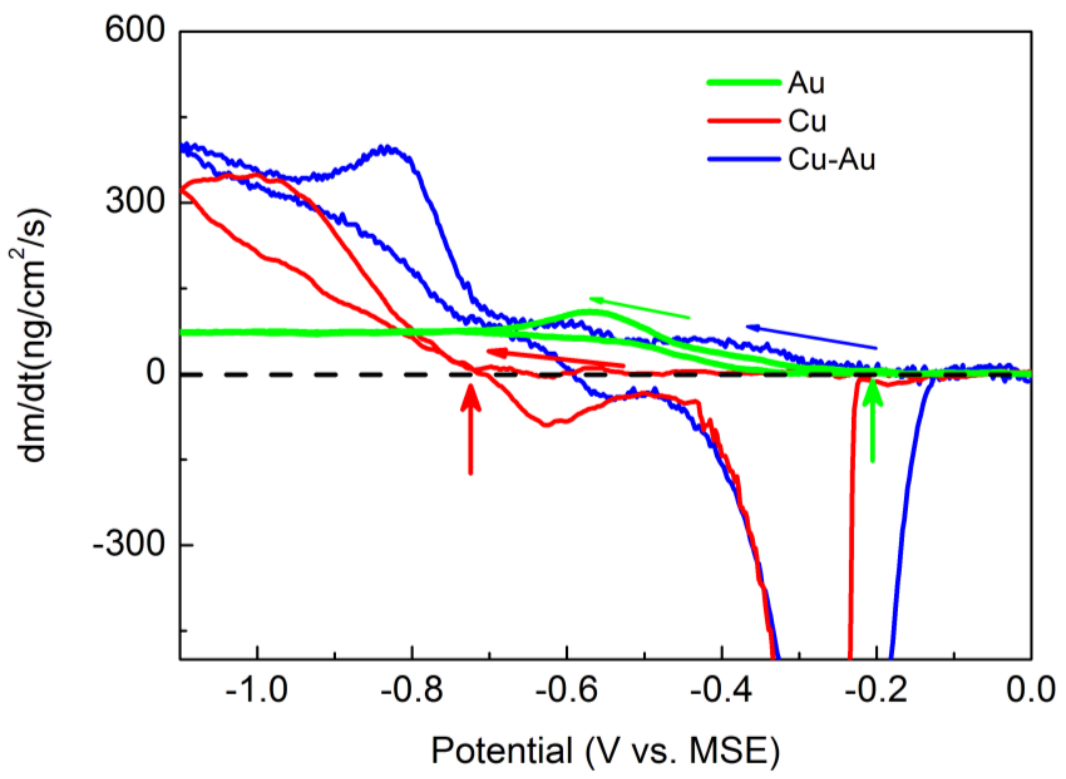

Figure 3.14 Comparison for the $\mathrm{dm} / \mathrm{dt}$ data for $\mathrm{Au}$ only, $\mathrm{Cu}$ only and $\mathrm{Au}+\mathrm{Cu}$.

reduction peak in the $\mathrm{Au}+\mathrm{Cu}$ solution, it is difficult to determine the exact onset of $\mathrm{Cu}$ co-deposition (blue line). It is clear however that $\mathrm{Cu}$ deposition is depolarized by co-deposition with $\mathrm{Au}$, shown both by the positive shifts of bulk $\mathrm{Cu}$ deposition and dissolution; the actual shift of co-deposition will be estimated combining composition data in the next section.

\subsubsection{Composition, morphology and crystal structure}

$\mathrm{Au}-\mathrm{Cu}$ alloy films of $\sim 200-300 \mathrm{~nm}$ are potentiostatically deposited on $\mathrm{Ru}$ substrates. Alloy composition as a function of potential is shown in Fig.3.15. Films are very uniform in composition, as indicated by the very small deviations. The sub-regular solution model is use to fit the portion having similar shape to the UPCD behavior shown above for the weakly complexed solution, and a redox potential of $-0.723 \mathrm{~V}$ is obtained, which is very close to the $-0.73 \mathrm{~V}$ determined by EQCM in Fig.3.12; meanwhile, two Margules parameters are obtained as well, which will be used for the construction of enthalpy of mixing later. The data points deviate from the fitting curve at more negative potentails may be due to kinetic effects, as larger overpotentials are needed to get corresponding composition [20]. Combining data from EQCM and 
composition, $\mathrm{Cu}$ deposition with $\mathrm{Au}$ is depolarized from $-0.73 \mathrm{~V}$ to more positive than $-0.53 \mathrm{~V}$, at which $\sim 14 \mathrm{at} . \%$ of $\mathrm{Cu}$ is already deposited. A precise assessment of the onset of co-deposition is made difficult by the slow deposition rate of Au rich films.

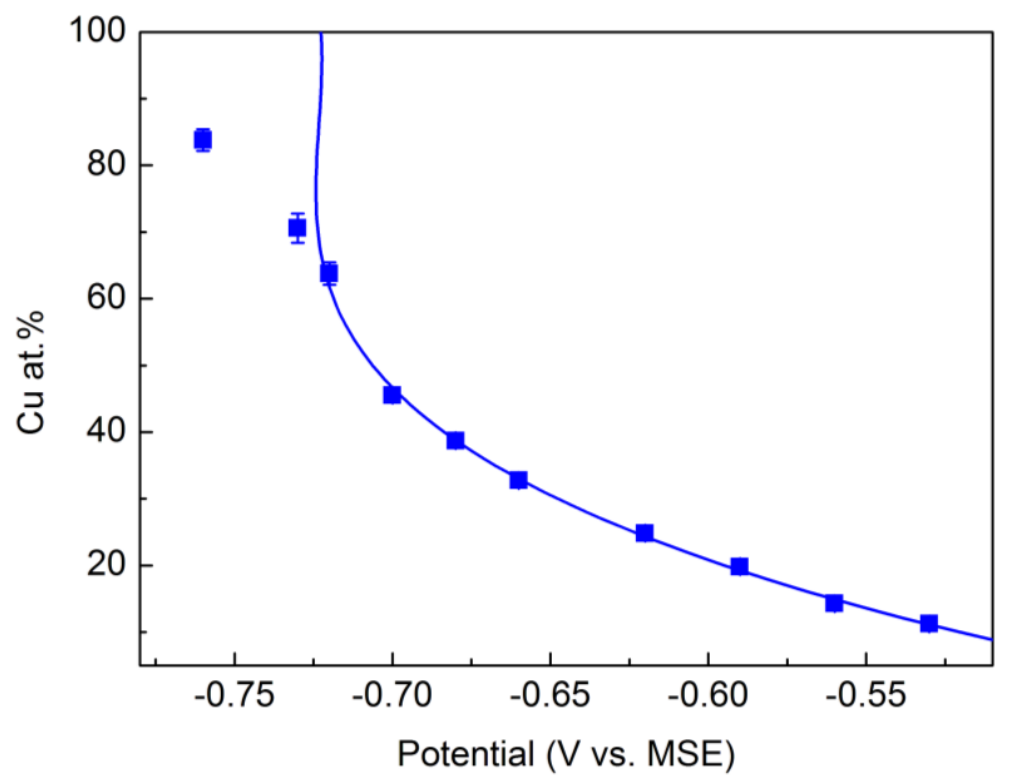

Figure 3.15 Composition (dots) as a function of deposition potential, $2 \mathrm{mM} \mathrm{Au}+20 \mathrm{mM} \mathrm{Cu}$. Error bars are based on two set of samples deposited on $\mathrm{Ru}$ and each sample at three different positions. The line is fitting by sub-regular solution model.

Optical images for films with various $\mathrm{Cu}$ contents are displayed in Fig.3.16. Films are smooth and shiny; with increasing $\mathrm{Cu}$ content, color changes from dark red to $\mathrm{Cu}$ red.

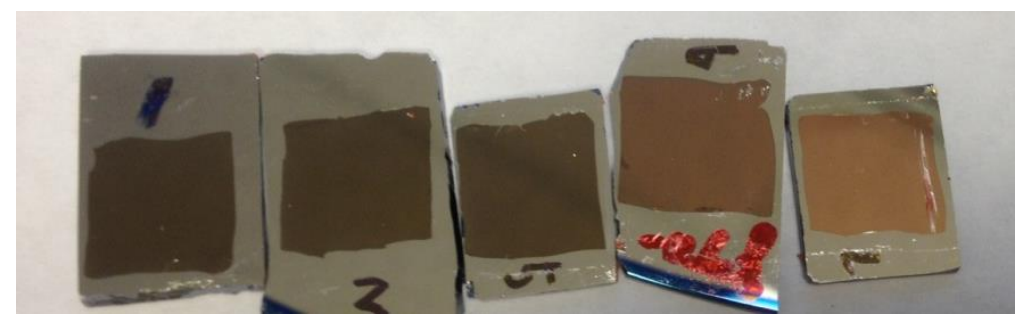

Figure 3.16 Optical pictures for $\mathrm{Cu}-\mathrm{Au}$ films with $\mathrm{Cu}$ content of: $23 \%, 30 \%, 52 \%, 66 \%$ and $85 \%$ (from left to right) 

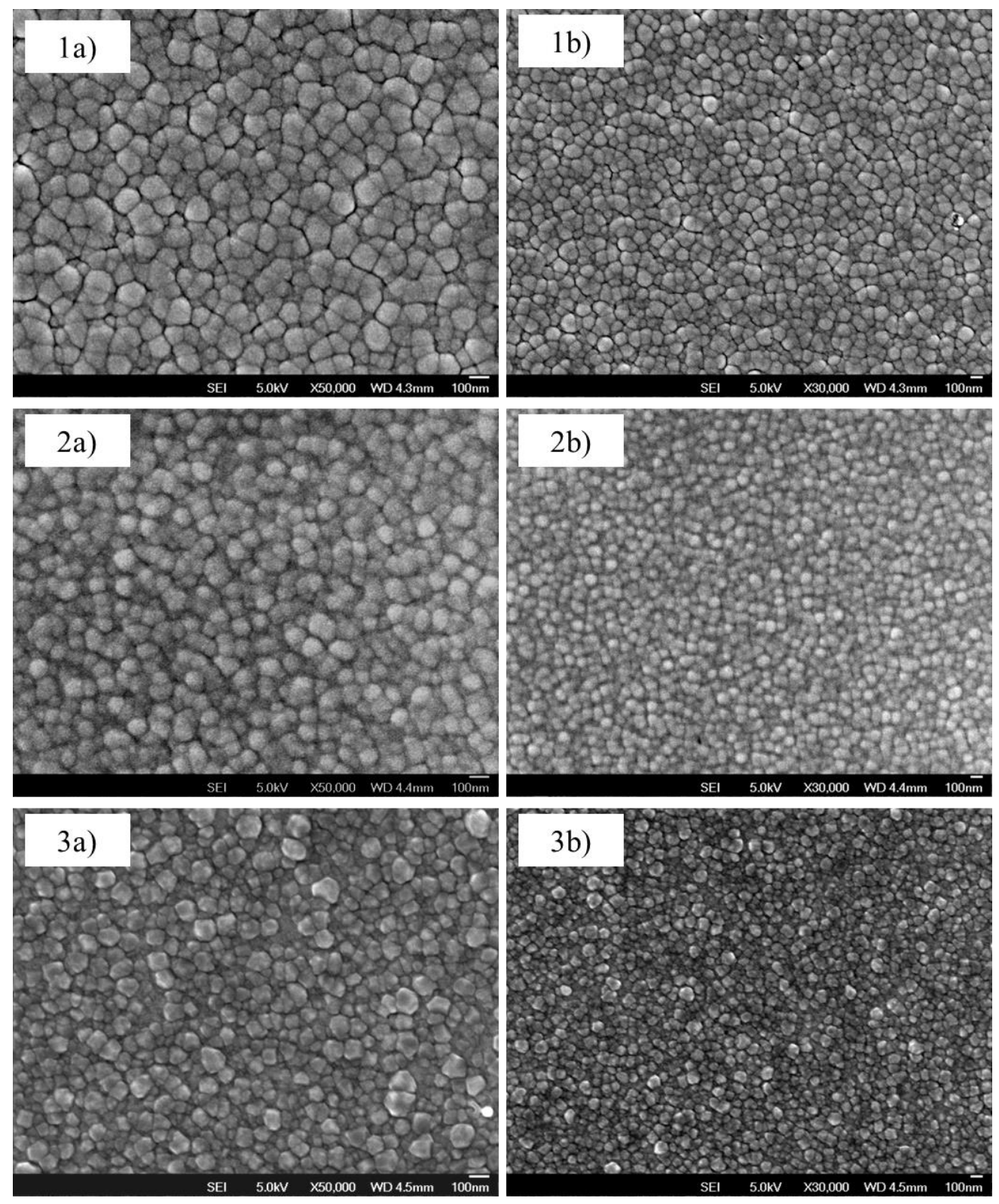

Figure 3.17 SEM images for Au-Cu films with various $\mathrm{Cu}$ contents: 1) 30at.\%; 2) 52at.\% and 3) 85at.\%; left with higher magnification and right lower magnification.

Corresponding SEM images for some of these films are displayed in Fig.3.17. Au rich films have larger apparent grain sizes and round grains; $\mathrm{Cu}$ rich films show smaller grains. Films deposited from this strongly complexed solution help to obtain smooth and stable morphology, greatly improved compared with respect to those from the weakly complexed solutions in Fig.3.6.

XRD patterns for $\mathrm{Au}-\mathrm{Cu}$ alloy with increasing $\mathrm{Cu}$ contents are shown in Fig.3.18. The 
(111) and (200) peaks both shift to higher angles with increasing $\mathrm{Cu}$, indicating continuous decrease in lattice parameters and the formation of a series of continuous solid solutions.

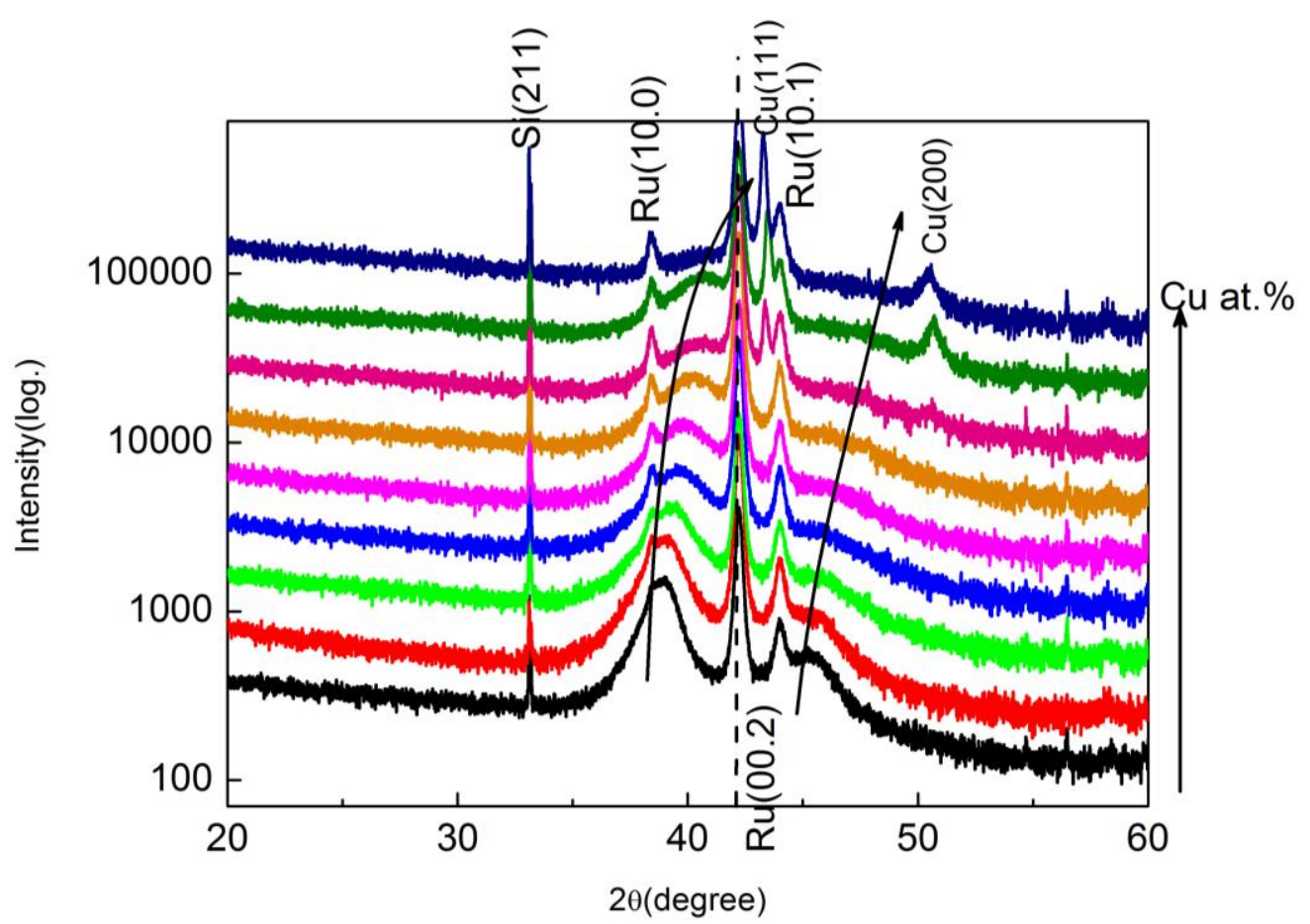

Figure 3.18 XRD patterns for $\mathrm{Au}-\mathrm{Cu}$ alloys with various $\mathrm{Cu}$ contents deposited on $\mathrm{Ru}$

To separate the peak for the films and the substrate peak, a double Voigt function is used to deconvolute and fit the (111) peak in Fig.3.18, and the peak position and width at half maximum are obtained and used to calculate the lattice parameter and grain size for the alloys, shown in Fig.3.19 and Fig.3.20, respectively. It is clearly shown that lattice constants agree very well with data from bulk alloys, with slight positive deviation from Vegards' law. This suggests limited or no precipitation of elemental phases. Grain size decreases with increasing $\mathrm{Cu}$ content up to about 33at.\% of $\mathrm{Cu}$, from $55 \mathrm{~nm}$ to $25 \mathrm{~nm}$, and then it remains roughly constant between 20 and 25 $\mathrm{nm}$. The trend agrees with what is shown by SEM images, but actual grain sizes are smaller than apparent ones, as they should be. 


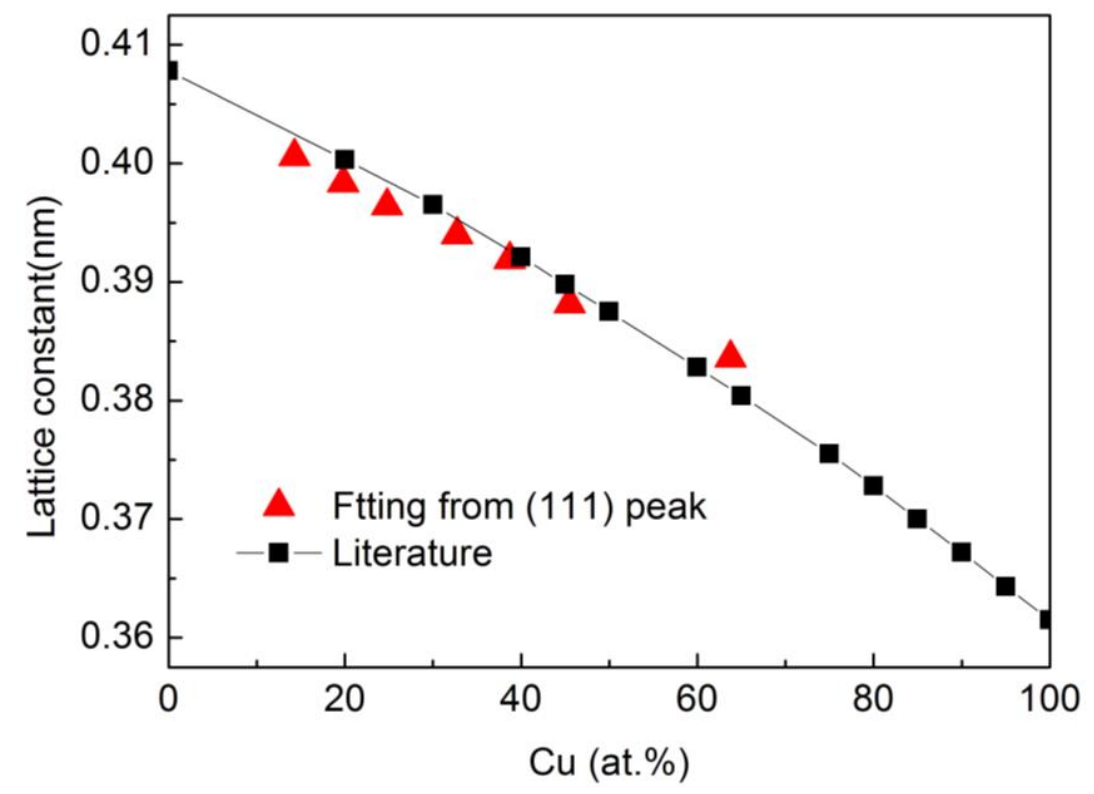

Figure 3.19 a) Lattice constant comparison with bulk alloy [16].

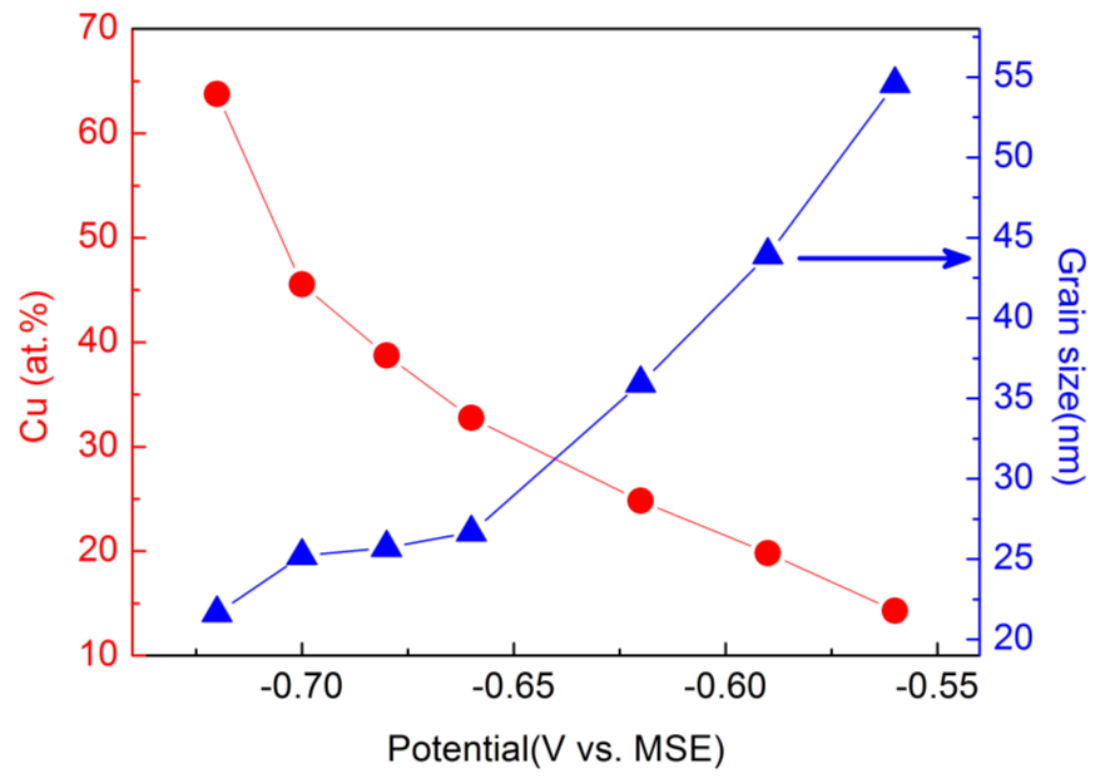

Figure 3.20 Grain size and composition as a function of deposition potential.

\subsection{Reverse UPCD: Au UPCD with Au}

As shown above for EN complexed solution, the redox potential for both $\mathrm{Cu}$ and $\mathrm{Au}$ can be tailored by complexation. Is it possible for example to make $\mathrm{Cu}$ more noble than $\mathrm{Au}$ and observe $\mathrm{Au}$ UPCD with $\mathrm{Cu}$ ? This will be answered in this section. As discussed in the experimental part, the sulfite based solution is made as follows: 0.168 $\mathrm{M} \mathrm{Na}_{2} \mathrm{SO}_{3}$ is used both as complexing agent and supporting electrolyte since the $\left[\mathrm{Na}^{+}\right]$ 
is already quite large and no other supporting electrolyte is needed. In order to complex Au, $20 \mathrm{mM} \mathrm{HAuCl}_{4}$ is added into sulfite solution; for $\mathrm{Au}+\mathrm{Cu}$ complexing, $20 \mathrm{mM} \mathrm{HAuCl}_{4}$ and $2 \mathrm{mM} \mathrm{CuSO}_{4}$ are added; the $0.168 \mathrm{M}$ sulfite is in large excess so that $\mathrm{Au}$ and $\mathrm{Cu}$ can be fully complexed. All solutions are kept at $\mathrm{pH}$ 8.0.

\subsubsection{Electrochemical study}

The CV and EQCM data for $\mathrm{Au}+$ sulfite are shown in Fig.3.21. It is well indicated that $\mathrm{Au}$ deposition starts at as negative as $-1.15 \mathrm{~V}$, compare with $0.3 \mathrm{~V}$ for the weakly complexed $\mathrm{HAuCl}_{4}$, leading to a polarization of $1450 \mathrm{mV}$ !
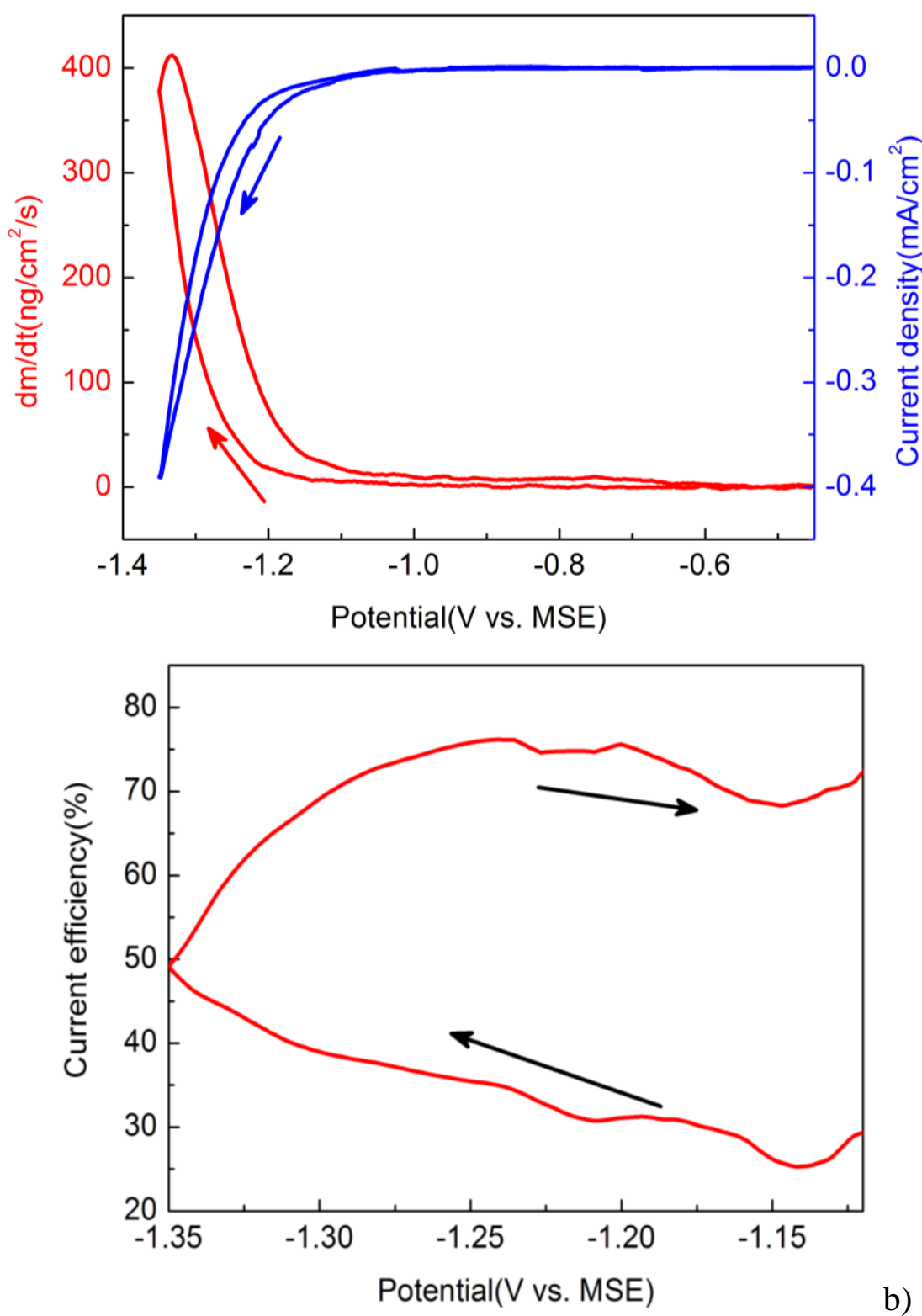

a)

b)

Figure $3.21 \mathrm{CV}$ and dm/dt data for sulfite $+20 \mathrm{mM} \mathrm{HAuCl}_{4}$ on Au resonator; scan rate of $5 \mathrm{mV} / \mathrm{s}$. 


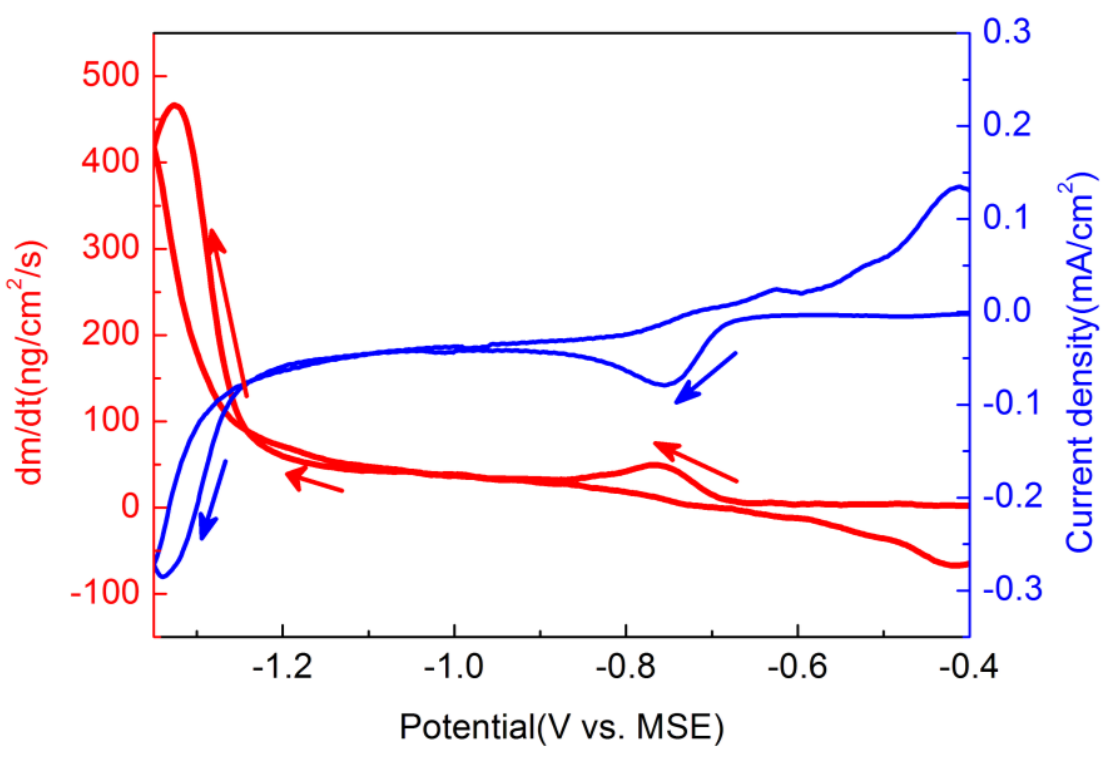

Figure $3.22 \mathrm{CV}$ and dm/dt data for sulfite $+20 \mathrm{mM} \mathrm{HAuCl}_{4}+2 \mathrm{mM} \mathrm{CuSO}_{4}$ on Au resonator; scan rate of $5 \mathrm{mV} / \mathrm{s}$.

The data for both $\mathrm{Au}+\mathrm{Cu}$ with sulfite are displayed in Fig.3.22. An increase in deposition rate is obsvered at $-0.65 \mathrm{~V}$, and a diffusion limited peak is reached at -0.74 $\mathrm{V}$ followed by a plateau from $-0.74 \mathrm{~V}$ to $-0.93 \mathrm{~V}$; a gradual increase in deposition rate is observed from $-0.92 \mathrm{~V}$ to $-1.2 \mathrm{~V}$, followed by drastic increase. Compared with only Au solution, deposition at potentials more positive than $-0.92 \mathrm{~V}$ is attributed to deposition of $\mathrm{Cu}$. To make better comparison, $\mathrm{dm} / \mathrm{dt}$ data are plotted together and shown in Fig.3.23. The dash line is drawn to show the diffusion limited deposition of $\mathrm{Cu}$. It can be clearly seen that deposition rate starts to increase at $-0.92 \mathrm{~V}$; this is co-deposition of Au with $\mathrm{Cu}$. 


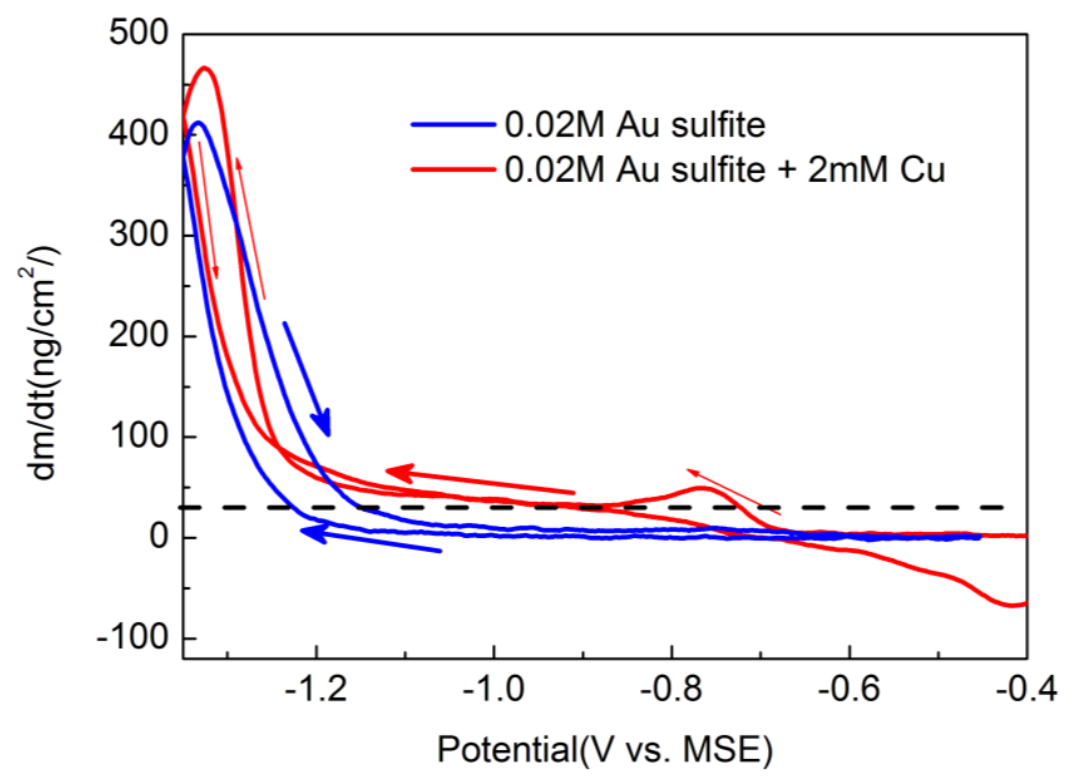

Figure 3.23 Comparison of $\mathrm{dm} / \mathrm{dt}$ data for $\mathrm{Au}$ only and $\mathrm{Au}+\mathrm{Cu}$ solutions.

\subsubsection{Composition, morphology and crystal structure}

Au-Cu films ( 200-300 nm) are potentiostatically deposited on $\mathrm{Ru}$. Composition vs. potential behavior is shown in Fig.3.24. It is shown that 10at.\% of Au is already deposited at $-0.95 \mathrm{~V}$, and 60 at.\% of $\mathrm{Au}$ is detected at $-1.20 \mathrm{~V}$, at which $\mathrm{Au}$ bulk deposition starts, confirming $\mathrm{Au}$ UPCD with $\mathrm{Cu}$, with a depolarization of approximately $250 \mathrm{mV}$. However, sulfur impurities are also detected in these films; it remains less than 5at.\% when $\mathrm{Au}$ is less than 60at.\%, and then saturates at 9at.\%. The incorporation of sulfur from electrodeposition of sulfite based solutions is common, e.g., in Au deposition [9] and Au-Ni deposition [21], and it is attributed to the disproportionation of $\mathrm{SO}_{3}{ }^{2-}$ to $\mathrm{SO}_{4}{ }^{2-}$ and elemental sulfur, the latter of which then is incorporated physically without electrochemical reaction. The fitting using the sub-regular solution model gives a redox potential of $-1.25 \mathrm{~V}$ for Au deposition, compared to $-1.20 \mathrm{~V}$ determined by EQCM. 


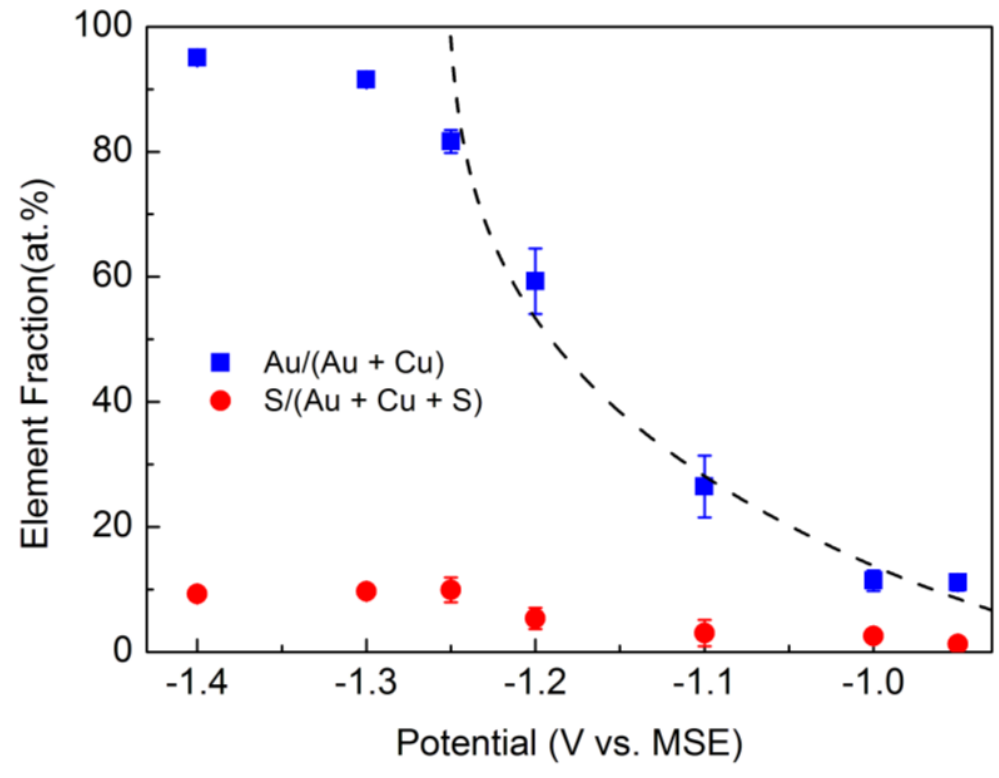

Figure 3.24 Composition as a function of potential for $\mathrm{Au}-\mathrm{Cu}$ alloys. Deviation is based on two sets of samples with each sample with three different measurements.

Films deposited from this sulfite solution are also very smooth and shiny, color changing from $\mathrm{Cu}$ red to $\mathrm{Au}$ yellow (Fig.3.25). In the SEM images (Fig.3.26), it is shown that films are very smooth, with apparent grains sizes less than $20 \mathrm{~nm}$.

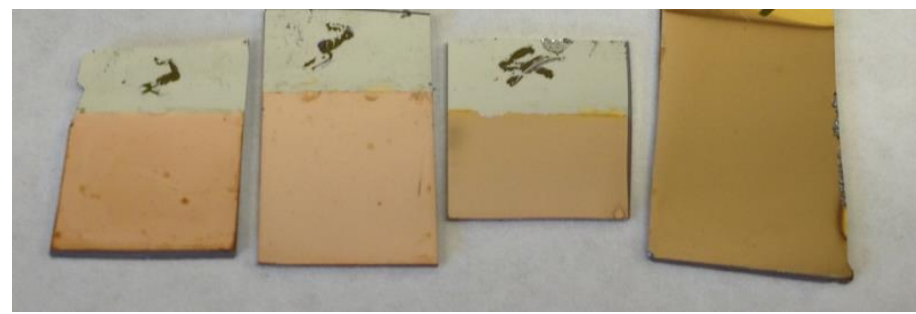

Figure 3.25 Optical images for Au-Cu alloys films with various $\mathrm{Au}$ content (from left to right): 11 at. $\%, 26$ at. $\%, 59$ at. $\%$ and 91 at. $\%$. 

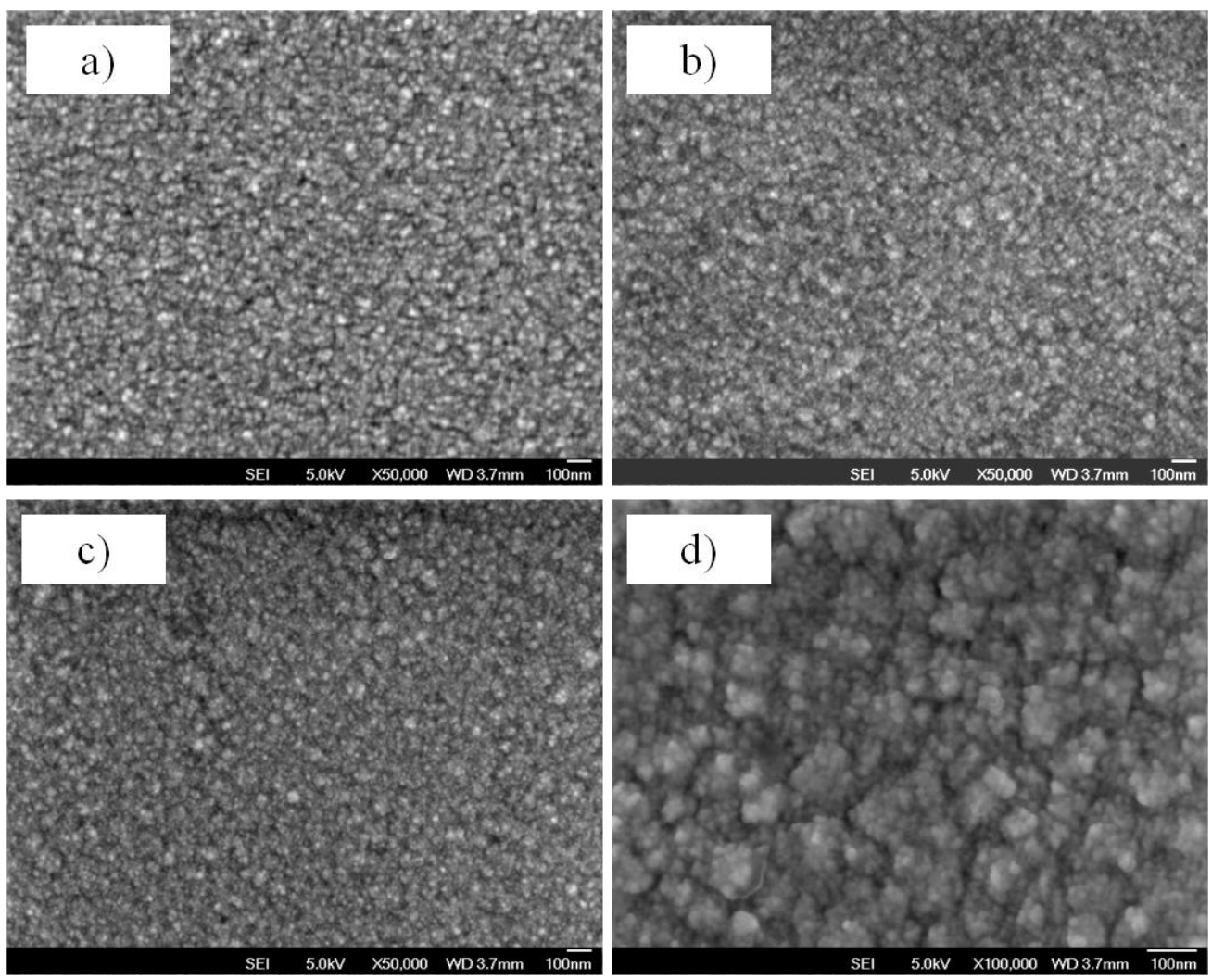

Figure 3.26. SEM images for Au-Cu alloys films with various Au content: a) 11at.\%; b) 26at.\%; c) 59at.\% and d) 91 at.\%.

XRD patterns for these $\mathrm{Au}-\mathrm{Cu}$ alloys are displayed in Fig.3.27. With decrease of deposition potentials and higher Au content, both the (111) and (200) peaks shift to lower angles, confirming the formation of a series of continuous solid solution. Fitting the alloy (111) peak allows the estimate of the lattice constants, which are shown in Fig. 3.28; they match well with the bulk alloy data. 


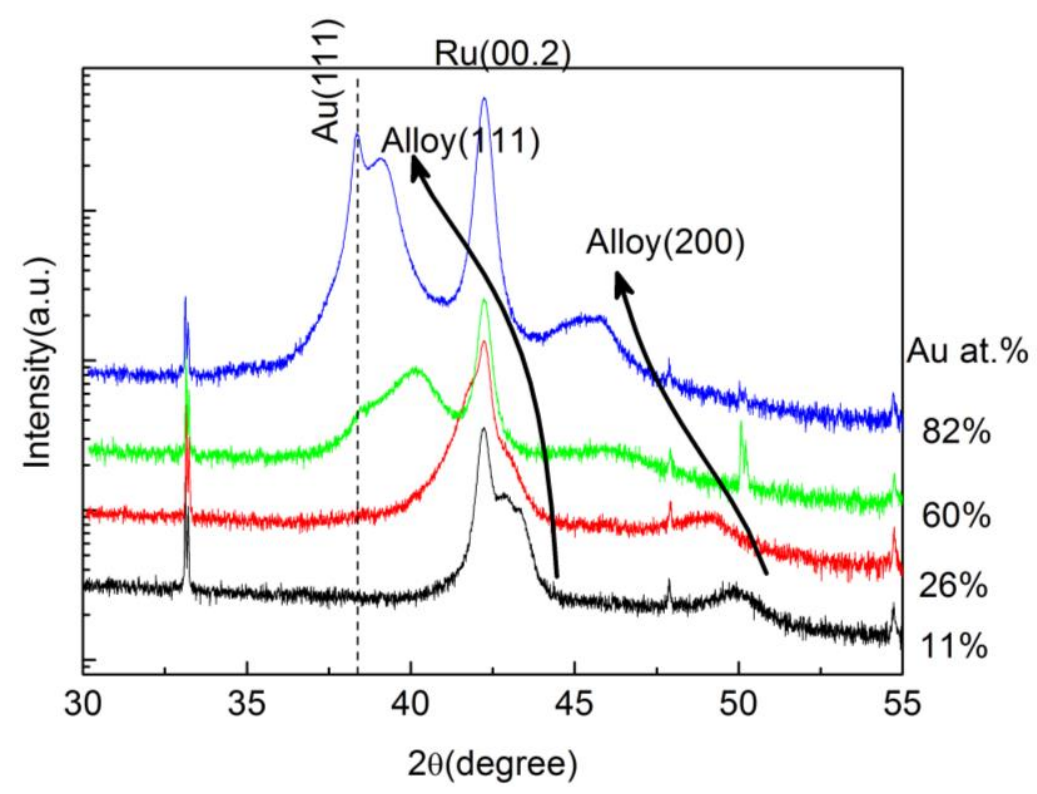

Figure 3.27 XRD patterns for Au-Cu alloys deposited from sulfite solution.

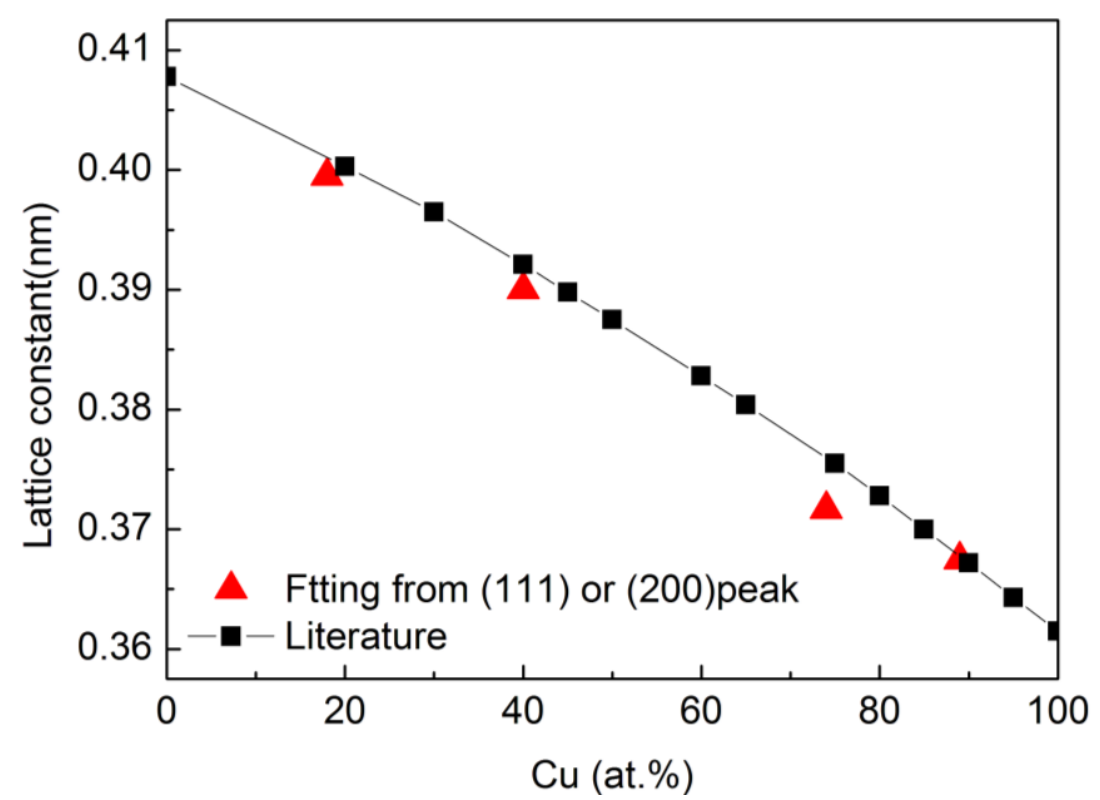

Figure 3.28 Lattice constant as a function of $\mathrm{Cu}$ content by fitting the (111) peak in Fig.3.27 compared with bulk alloy data [16].

\subsection{Enthalpy of Mixing}

The enthalpy of mixing for $\mathrm{Au}-\mathrm{Cu}$ alloys deposited from the three different electrolytes shown above is calculated using the sub-regular solution model. The Margules parameters determining the mixing enthalpy were obtained by fitting the composition vs. potential plots shown in Fig.3.4, Fig.3.15 and Fig.3.21, respectively. In the case of Au UPCD with $\mathrm{Cu}$ alloys, we assume that sulfur is physically 
incorporated without electrochemical reaction and therefore has limited effect on the enthalpy of alloy formation. The data are plotted in Fig.3.29 together with those determined for bulk alloys obtained by metallurgical procedures [2]. In the case of $\mathrm{Cu}$ UPCD with Au from weakly complexed solution, the minimum in mixing enthalpy appears at $\sim 38$ at. $\%$ of $\mathrm{Cu}$, with an average around $-7.1 \mathrm{~kJ} / \mathrm{mol}$; for $\mathrm{Cu}$ UPCD with $\mathrm{Au}$ from EN complexing solution, the minimum occurs at $\sim 35$ at. $\%$ of $\mathrm{Cu}$, similar to that from weakly complexed solution, while the value of this minimum is $\sim-7.8 \mathrm{~kJ} / \mathrm{mol}$. In contrast with both $\mathrm{Cu}$ UPCD cases, the minimum of the enthalpy of mixing of alloys obtained via $\mathrm{Au}$ UPCD with $\mathrm{Cu}$ appears in the $\mathrm{Au}$-rich region at $\sim 57 \mathrm{at} . \%$ of $\mathrm{Cu}$, with a value of $-5.85 \mathrm{~kJ} / \mathrm{mol}$. Interestingly, the latter value is very similar to that observed in bulk alloys, that is $-5.88 \mathrm{~kJ} / \mathrm{mol}$ at 62 at. $\%$ of $\mathrm{Cu}$.

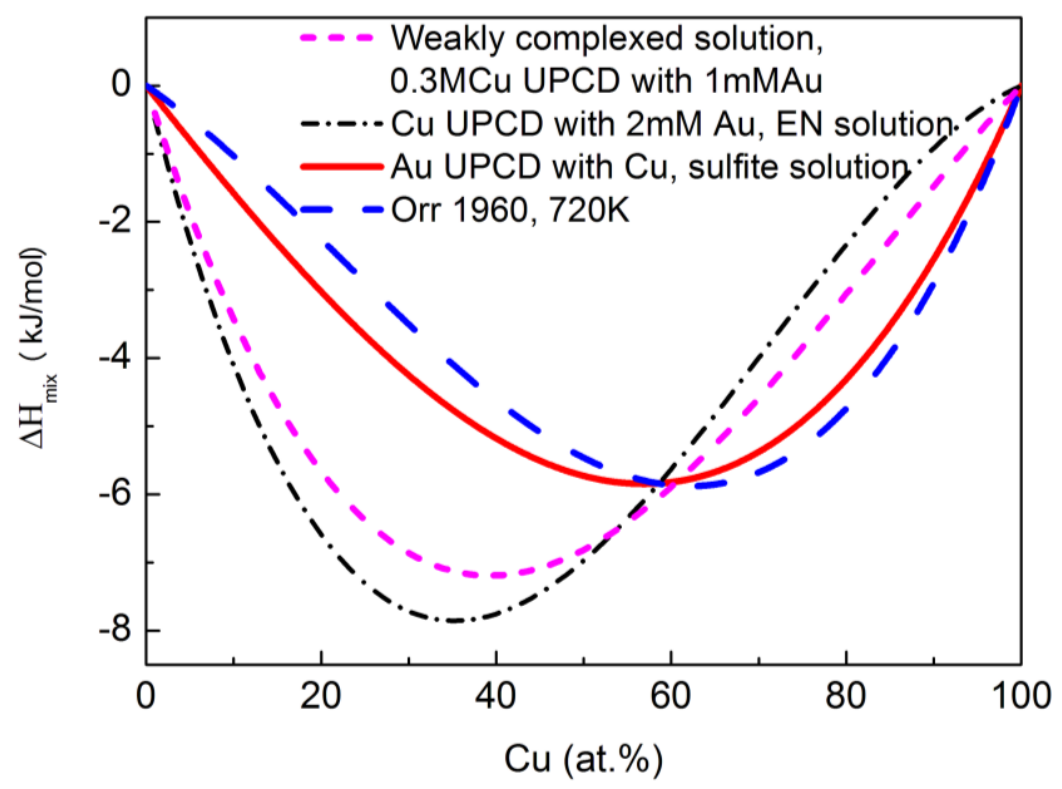

Figure 3.29 Enthalpy of mixing comparison among alloy: $\mathrm{Cu}$ UPCD with Au from weakly complexed solution, $\mathrm{Cu}$ UPCD with Au from EN complexed solution, Au UPCD with $\mathrm{Cu}$ from sulfite complexed solution and bulk alloys [2].

The discrepancy among the various enthalpy trends may be due to variations of the degree of short range ordering in these alloys [2]; the higher the degree of short range ordering, the larger is the enthalpy minimum [2]. No difference in crystal structure is observed among the various alloys, since XRD shows only a random FCC structure in all of them. However, XRD averages information on atomic positions over areas of 
$\mu \mathrm{ms}$ and therefore it is not sensitive to nano scale short range ordering features, and is unable to provide details about any possible difference in the short range ordering of these alloys.

\subsection{Conclusion}

$\mathrm{Cu}$ UPCD with $\mathrm{Au}$ was observed in solutions with both weak complexation for $\mathrm{Au}$, and strong complexation for both $\mathrm{Au}$ and $\mathrm{Cu}$; films from the former solution are powdery and rough, while those from the latter are very smooth with smaller grain sizes. Reverse UPCD, Au UPCD with $\mathrm{Cu}$ is demonstrated from sulfite complexed solution for both $\mathrm{Au}$ and $\mathrm{Cu}$; obtained films are also very smooth and stable in morphology. Enthalpy of mixing obtained by fitting the experimental composition vs. potential behaviors is more or less comparable to bulk alloy data in magnitude, but with a minimum observed for Au rich alloys for Cu UPCD with Au process, and for $\mathrm{Cu}$ rich alloys for $\mathrm{Au}$ UPCD with $\mathrm{Cu}$ process. The minimum from $\mathrm{Au}$ UPCD with $\mathrm{Cu}$ is similar in both magnitude and composition to the minimum for bulk alloys. 


\section{References}

[1] T.A. Green, Gold Bull. 40 (2007) 105.

[2] R.L. Orr, Acta Metall. 8 (1960) 489.

[3] B. Sundman, S.G. Fries, W.A. Oates, Calphad 22 (1998) 335.

[4] D.M. Kolb, M. Przasnyski, H. Gerischer, J. Electroanal. Chem. Interfacial Electrochem. 54 (1974) 25.

[5] E. Herrero, L.J. Buller, H.D. Abruña, Chem. Rev. 101 (2001) 1897.

[6] J.J. Mallett, W. Shao, D. Liang, G. Zangari, Electrochem. Solid-State Lett. 12 (2009) D57.

[7] D. Lu, K. Tanaka, J. Electroanal. Chem. 430 (1997) 69.

[8] S. Zhu, W. Gorski, D.R. Powell, J.A. Walmsley, Inorg. Chem. 45 (2006) 2688.

[9] Y. Okinaka, Gold Bull. 31 (1998) 3.

[10] U. Koelle, A. Laguna, Inorg. Chim. Acta 290 (1999) 44.

[11] M. J-Liew, S. Sobri, S. Roy, Electrochimi. Acta 51 (2005) 877-881.

[12] V.N. Shibanov, E.A. Khadykina, Russ. J. Appl. Chem. 75 (2002) 1740.

[13] D.A. Buttry, M.D. Ward, Chem. Rev. 92 (1992) 1355.

[14] Y. Liu, D. Gokcen, U. Bertocci, T.P. Moffat, Science 338 (2012) 1327.

[15] D. Liang, J.J. Mallett, G. Zangari, J. Electrochem. Soc. 158 (2011) D149.

[16] H. Okamoto, D.J. Chakrabarti, D.E. Laughlin, T.B. Massalski, Bull. Alloy Phase Diagrams 8 (1987) 454.

[17] W. Shao, G. Zangari, J. Phys. Chem. C 113 (2009) 10097.

[18] L. Sillen, L. G., Gunnar, Stability Constants of Metal-Ion Complexes, 2nd Ed.., 1964.

[19] S. Aksu, F.M. Doyle, J. Electrochem. Soc. 148 (2001) B51. 
[20] J.J. Mallett, E.B. Svedberg, J.E. Bonevich, A.J. Shapiro, W.F. Egelhoff, T.P. Moffat, J. Electrochem. Soc 155 (2008) D1.

[21] E. Rouya, G.R. Stafford, U. Bertocci, J.J. Mallett, R. Schad, M.R. Begley, R.G. Kelly, M.L. Reed, G. Zangari, J. Electrochem. Soc. 157 (2010) D396. 


\section{Chapter 4 Electrodeposition of Fe-Pt alloys from an}

\section{alkaline solution}

\section{Overview}

In Chapter one, we briefly introduced the thermodynamics of Fe-Pt, mainly its equilibrium phase diagram. One of its three ordered structures, the equiatomic $\mathrm{Fe}-\mathrm{Pt}$ with $\mathrm{L} 1_{0}$ structure is a highly promising material for magnetic recording media due to its high magnetic anisotropy [1,2]. Deposition of Fe-Pt alloy films has been extensively researched using various deposition techniques, e.g., sputtering [3], wet chemistry [4], electrodeposition [5,6] and MBE [7]. Regardless of deposition methods, the as-deposited Fe-Pt alloys prepared at relatively low temperatures form the random face centered cubic (FCC) structure due to kinetic hindrance to the formation of the ordered high anisotropy phase. Therefore, post--annealing at high temperatures $\left(\sim 600^{\circ} \mathrm{C}\right)$ is usually needed to achieve the properties sought for.

Electrodeposition of Fe-Pt shows some advantages over vacuum techniques in that it is excellent in filling patterns with high aspect ratios [8]. For electrodeposition of $\mathrm{Fe}-\mathrm{Pt}$, previous efforts of synthesizing Fe-Pt films using simple acidic electrolytes have met only with partial success. Acidic chloride or sulfate/chloride electrolytes and potentiostatic $[5,9,10]$, or pulse plating [11] methods have been used to grow Fe-Pt of various compositions. The as-deposited films were observed to exhibit an FCC or body centered cubic (BCC) structure, depending on composition, and to contain large amount of incorporated oxygen, up to 30 at. \% near the equiatomic composition [11]. The dependence of alloy composition on potential was described by the UPCD model [5], whereby the less noble metal was co-deposited in the alloy at a potential more positive than that required for the reduction of the pure metal onto itself due to the negative enthalpy of mixing with the formation of solid solutions of $\mathrm{Fe}-\mathrm{Pt}$, similar to what has been shown in chapter 3 for $\mathrm{Au}-\mathrm{Cu}$. In these electrolytes, $\mathrm{Fe}$ co-deposition 
was observed to occur under diffusion limited conditions for Pt reduction [5,11], which potentially favors morphological instabilities and the formation of dendritic or porous films. The large amount of oxygen incorporated in the films has been ascribed to the strong hydrogen evolution during deposition, which causes a local $\mathrm{pH}$ increase at the interface and thus favors hydrolysis and precipitation of $\mathrm{Fe}^{2+}$ and $\mathrm{Fe}^{3+}$ oxides or hydroxides [10]. Even if absent from the original electrolyte formulation, $\mathrm{Fe}^{3+}$ can in fact be generated by oxidation of $\mathrm{Fe}^{2+}$ in the presence of oxygen [12] or at the anode, and tends to precipitate at lower $\mathrm{pH}$ values than the latter due to the much smaller solubility. Electrodeposition from acidic, non-complexed electrolytes would therefore result in porous rough films with a low saturation magnetization and little control over the microstructure. An additional consequence of oxygen incorporation in the films is the need for a high annealing temperature and a reducing atmosphere to achieve the $\mathrm{L1}_{0}$ structure and high anisotropy of interest $[18,19]$, further complicating the overall process.

In this chapter, we will demonstrate an electrochemical process (Table 4.1 for the electrolyte) developed in our group that has shown some progress with respect to the solutions discussed above [13].

Most of the previous attempts to grow Fe-Pt have made use of Pt chloride complexes as the Pt source. The large difference between the redox potential of $\mathrm{Pt}$ from chlorides $\left(\mathrm{PtCl}_{6}^{2-}\left(\right.\right.$ or $\left.\left.P t C l_{4}\right) / \mathrm{Pt}, \quad E^{0}=0.758 V_{S S E}\right)$ and of $\mathrm{Fe}\left(\mathrm{Fe}^{2+} / \mathrm{Fe}, E^{0}=-0.44 V_{S S E}\right)$ would likely result in co-deposition to occur under diffusion limited conditions for Pt, thus triggering the formation of porous, dendritic films. In order to overcome this problem, we used the diamino dinitrite complex of Pt, $\mathrm{Pt}\left(\mathrm{NH}_{3}\right)_{2}\left(\mathrm{NO}_{2}\right)_{2}$ [14], or Pt-p-salt, to further shift cathodically the $\mathrm{Pt}$ reduction reaction. $\mathrm{Pt}$ in this complex is in the $\mathrm{Pt}^{2+}$ form, and its reduction would require half the charge required for the reduction from $\mathrm{PtCl}_{4}$ or $\mathrm{H}_{2} \mathrm{PtCl}_{6}$. Electrodeposition of Pt from its p-salt exhibits a sharp drop in efficiency at temperatures below $60^{\circ} \mathrm{C}$; furthermore, efficiency decreases 
approximately linearly with $\mathrm{pH}$ due to the increasing rate of hydrogen evolution: this process therefore requires an alkaline $\mathrm{pH}$ to proceed at an appreciable rate [22].

In an alkaline environment, however, either ferrous or ferric ions would tend to hydrolyze and precipitate as oxides or hydroxides, while the latter is easier to precipitate out with smaller solubility of its hydroxide; a combination of citrate and glycine was therefore used to complex Fe while at the same time buffering the $\mathrm{pH}$ value around 8. The citrate anion is an organic compound derived from citric acid $\mathrm{H}_{3} \mathrm{C}_{6} \mathrm{H}_{5} \mathrm{O}_{7}$, which contains three carboxylic groups, capable to bind transition metals, thus preventing their possible precipitation in alkaline conditions; at $\mathrm{pH} 8$ in particular the citrate ion is stable in the form $\mathrm{C}_{6} \mathrm{H}_{5} \mathrm{O}_{7}^{3-}$ [15]. Glycine $\mathrm{NH}_{2} \mathrm{CH}_{2} \mathrm{COOH}$ on the other hand contains both an amine and a carboxylic group as potential complexing functional groups, and is known to act as a buffer in the $\mathrm{pH}$ range 8.8 to 10.6. The combination of citrate and glycine leads to an enhanced complexing strength towards $\mathrm{Fe}$, as will be shown later in the next section. Citrate complexes the $\mathrm{Fe}^{3+}$ ion more strongly than $\mathrm{Fe}^{2+}$ (see Table 4.2); the ferric ion was therefore preferred in the electrolyte formulation to enhance electrolyte stability. The use of $\mathrm{Fe}^{2+}$ would in principle be more advantageous since its reduction would require a 2-electron exchange and would therefore be more efficient; however, the lower strength of complexation and the possibility for $\mathrm{Fe}^{2+}$ to be oxidized and/or to form hydroxides during operation, has led us to use $\mathrm{Fe}^{3+}$ instead. Besides, the presence of $\mathrm{Fe}^{2+}$ could result in partial oxidation to $\mathrm{Fe}^{3+}$ [12] by dissolved oxygen and/or at the anode during deposition, and to an unknown concentration of the various species in solution.

Finally, ammonium sulfate and sodium nitrite were added to enhance electrolyte stability; Pt complexes in fact are known to decompose in operation, with the complexing groups being slowly replaced over time by aquo complexes $[16,17]$.

For a clear overview of the chapter, this chapter will be discussed in four sections: 1) electrochemistry of co-deposition of Fe-Pt alloys; 2) magnetic and structural 
characterization of near equiatomic FePt single layer films; 3) bi-layer and multilayer structures; 4) microstructure study of near equiatomic Fe-Pt as well as $\mathrm{Fe}$ rich Fe-Pt films, the latter with focus on $\mathrm{Fe}_{3} \mathrm{Pt}$.

\subsection{Electrochemistry of co-deposition of Fe-Pt alloys}

\subsubsection{Bath and experimental conditions}

The electrolyte used in this investigation is reported in Table 4.1; the concentration of ammonium citrate and glycine was $0.15 \mathrm{M}$ in the equilibria calculations and in all the electrochemical studies, while a 0.1 or $0.2 \mathrm{M}$ concentration was used in some cases to grow Fe-Pt films for comparison. An acidic electrolyte was used for comparison purposes, as discussed in detail in the text. In the following we will refer to the alkaline solution without either Fe or Pt salts as the background solution. $\mathrm{pH}$ of the solution was adjusted by adding dilute $\mathrm{KOH}$ or $\mathrm{H}_{2} \mathrm{SO}_{4}$ to $8.0 \pm 0.1$. Experiments were performed using a three-electrode cylindrical cell in the vertical configuration, heated at a constant temperature of $75^{\circ} \mathrm{C}$ (two set of films deposited at $65^{\circ} \mathrm{C}$ or $85^{\circ} \mathrm{C}$ for comparison of deposition rates) by a hot water jacket circulator (EX 7). The counter-electrode was a Pt mesh, and the reference was a saturated mercurous sulfate electrode (SSE), separated from the main heated compartment by a Luggin capillary in order to be able to operate the reference electrode close to room temperature, despite the elevated temperature of the plating solution. In the following all potentials

will be referred to $\operatorname{SSE}\left(E_{S S E}=0.650 V_{S H E}\right.$ ) at room temperature. No stirring or de-aeration was used in any experiment.

For EQCM experiments, as already shown in Chapter 2, Au coated AT-cut quartz resonators with $0.5 \mathrm{~cm}$ diameter $(0.196 \mathrm{~cm} 2$ working area) and a resonant frequency of $9 \mathrm{MHz}$ were used. All CVs in this chapter were carried out using EQCM with $\mathrm{Au}$ resonator at a scan rate of $20 \mathrm{mV} / \mathrm{s}$ unless specified. 
For deposition of films, substrates of $100 \mathrm{~nm} \mathrm{Ru}$ layer, with preferential (00.1) orientation, sputtered on $5 \mathrm{~nm}$ Ta adhesion layer on top of native oxide of $\mathrm{Si}$ (001) wafer were used.

Alloy composition was determined by energy dispersive spectroscopy EDS using a detector attached to a JEOL JSM-6700F scanning electron microscope. The ratio Fe to Pt content was determined using an accelerating voltage of $15 \mathrm{kV}$ and the $\mathrm{X}$-ray $\mathrm{K}$ line for $\mathrm{Fe}$, the $\mathrm{M}$ line for Pt. Using a high accelerating voltage however results in the low energy oxygen-emitted X-rays being generated deep in the sample and getting strongly absorbed. The relative oxygen content $\mathrm{x}_{\mathrm{O}} /\left(\mathrm{x}_{\mathrm{O}}+\mathrm{x}_{\mathrm{Fe}}+\mathrm{x}_{\mathrm{Pt}}\right)$ was therefore determined using an accelerating voltage of $5 \mathrm{kV}$. Some of these measurements were validated using Auger electron spectroscopy (AES, Perkin-Elmer PHI 660 Scanning Auger Multiprobe), obtaining a good agreement across the whole range of compositions. Surface topography of selected films was investigated using an atomic force microscope AFM (Digital Instruments, Dimension 3100) in tapping mode.

The surface area measured by AFM is approximate since this instrument cannot correctly image features with higher aspect ratio than the tip. Detecting any fine porosity is a particular challenge for AFM as it is difficult for the scanning probe to penetrate into the internal structure. For this reason, the real surface area of selected $\mathrm{Pt}$ films was quantified by the underpotential deposition (UPD) of hydrogen, whereby the charge $\mathrm{Q}_{\mathrm{H}}$ measured upon electrosorption of hydrogen under UPD conditions on Pt and the real surface area is determined dividing $\mathrm{Q}_{H}$ by the UPD charge of $210 \mu \mathrm{C} / \mathrm{cm}^{2}$, calculated for an atomically smooth Pt surface $[18,19]$ This method has been extended to estimate the relative surface area of Fe-Pt alloy films: in this case the charge under the hydrogen UPD wave was evaluated after dissolution of the surface Fe by CV. Hydrogen UPD at Pt or Fe-Pt electrodes electroplated either from alkaline complexing solutions or acidic electrolytes was carried out in a $1 \mathrm{M} \mathrm{H}_{2} \mathrm{SO}_{4}$ solution, deaerated for 40 min by sparging $\mathrm{N}_{2}$ before the experiments, by performing successive CVs at a scan 
rate of $20 \mathrm{mV} / \mathrm{s}$ for Fe-Pt films and 50mV/s for Pt films, in the voltage window between the open circuit potential and $-0.66 \mathrm{~V}_{\mathrm{SSE}}$. The hydrogen UPD was measured by separately integrating the cathodic and the anodic current between -0.4 and $-0.66 \mathrm{~V}$. The integrated charge was always slightly larger for the cathodic than for the anodic sweep, despite solution deaeration; the difference was attributed to the oxygen reduction reaction.

\subsubsection{Preparation of Pt $\mathrm{p}$ salt}

Pt p salt is made from acidic $\mathrm{H}_{2} \mathrm{PtCl}_{6}$ following the procedure in Ref. [20]. Briefly, $1 \mathrm{~g}$ of $\mathrm{H}_{2} \mathrm{PtCl}_{6}$ and $4 \mathrm{~g}$ of $\mathrm{NaNO}_{2}$ are dissolved into $100 \mathrm{ml}$ of deionized water (DI water, $18.2 \mathrm{M} \Omega . \mathrm{cm}$ ) and heated to $95^{\circ} \mathrm{C}$ until the solution becomes colorless from original brown red (it takes $\sim 9 \mathrm{~h}$ ); $4 \mathrm{~mL}$ of $14 \mathrm{M}$ of $\mathrm{NH}_{3} \cdot \mathrm{H}_{2} \mathrm{O}$ is added into the solution, which is kept in a refrigerator. An off-white precipitate flocculates out of the solution; it is filtered while continuously rinsed with DI water to wash away remaining $\mathrm{NH}_{3} \cdot \mathrm{H}_{2} \mathrm{O}$, dried and used.

Table 4.1 Make-up of the Fe-Pt electrolyte.

$\begin{array}{cc}\mathrm{Pt}\left(\mathrm{NH}_{3}\right)_{2}\left(\mathrm{NO}_{2}\right)_{2} & 15 \mathrm{mM} \\ \mathrm{Fe}_{2}\left(\mathrm{SO}_{4}\right)_{3} & 50 \mathrm{mM} \\ \left(\mathrm{NH}_{4}\right)_{2} \mathrm{C}_{6} \mathrm{H}_{6} \mathrm{O}_{7} & 0.15 \mathrm{M} \\ \mathrm{NH}_{2} \mathrm{CH}_{2} \mathrm{COOH} & 0.15 \mathrm{M} \\ \mathrm{NaNO}_{2} & 0.1 \mathrm{M} \\ \left(\mathrm{NH}_{4}\right)_{2} \mathrm{SO}_{4} & 0.1 \mathrm{M}\end{array}$

\subsubsection{Preparation of the electrolyte}

After adding all the chemicals in the solution, slowly adjust the $\mathrm{pH}$ to $\sim \mathrm{pH} 8$. The 
solution will become cloudy as it takes time for the complexation of $\mathrm{Fe}^{3+}$ with citrate/glycine to fully establish. It is necessary to heat the solution to $\sim 70^{\circ} \mathrm{C}$ until it becomes clear; cool it down to room temperature, and it is found that $\mathrm{pH}$ is lower than $\mathrm{pH} 8$. Adjust again to $\mathrm{pH}$ to $\sim 8$, and repeat the cycle of heating and cooling. It usually takes about four cycles then it is possible to make a transparent $\mathrm{pH} 8$ solution.

\subsubsection{Solution chemistry}

In order to better understand the solution chemistry, we determined the equilibrium concentrations of the various species in solution by solving the system of equations for the relevant equilibria, as summarized in Table 4.2 [21]. Equilibria for the ferrous ion are also calculated separately even if $\mathrm{Fe}^{2+}$ is not present in the initial formulation; as will be discussed in the following these are of interest since $\mathrm{Fe}^{2+}$ is generated at the electrode by the one-electron reduction of $\mathrm{Fe}^{3+}$ with a concentration at the interface which is assumed to be approximately equal to that of $\mathrm{Fe}^{3+}$ when this reaction is diffusion limited. Accordingly, the concentrations of both $\mathrm{Fe}^{2+}$ and $\mathrm{Fe}^{3+}$ species used in the equilibrium calculations are assumed to be $0.1 \mathrm{M}$ in the separate calculation even though only $\mathrm{Fe}^{3+}$ was used for the experiments. Eqs. (1-10) represent the equilibria of $\mathrm{Fe}^{2+}$ or $\mathrm{Fe}^{3+}$ with citrate or among the various citrate species, eqs. (13-20) the equilibria between the Fe ions and glycine, or among the various glycine species; eqs. (11-12) the ammonium equilibrium and the association of Fe with ammonia, and finally eqs. (21-22) consider the possibility of $\mathrm{Fe}$ hydroxide formation. The equilibrium constant for these reactions were taken from Refs. [22-25] as specified in Table 4.2. Reactions leading to the formation of hybrid citrate-glycinate complexes were disregarded, and the equilibria involving Pt ions were neglected; in particular, $\mathrm{Pt}$ may form complexes with citrate but, due to its low concentration, this reaction would not affect the overall citrate availability significantly. Finally, in order to attain reliable solutions to the set of equations, the equilibria involving $\mathrm{Fe}^{2+}$ were solved separately from those involving $\mathrm{Fe}^{3+}$, assuming both $0.1 \mathrm{M}$ in corresponding calculation. 
Table 4.2 Equilibria and stability constants. All constants were from Ref. [22] unless specified.

Association or Deionization

Equilibria Equations
Equilibrium

Constants
Equation

No.

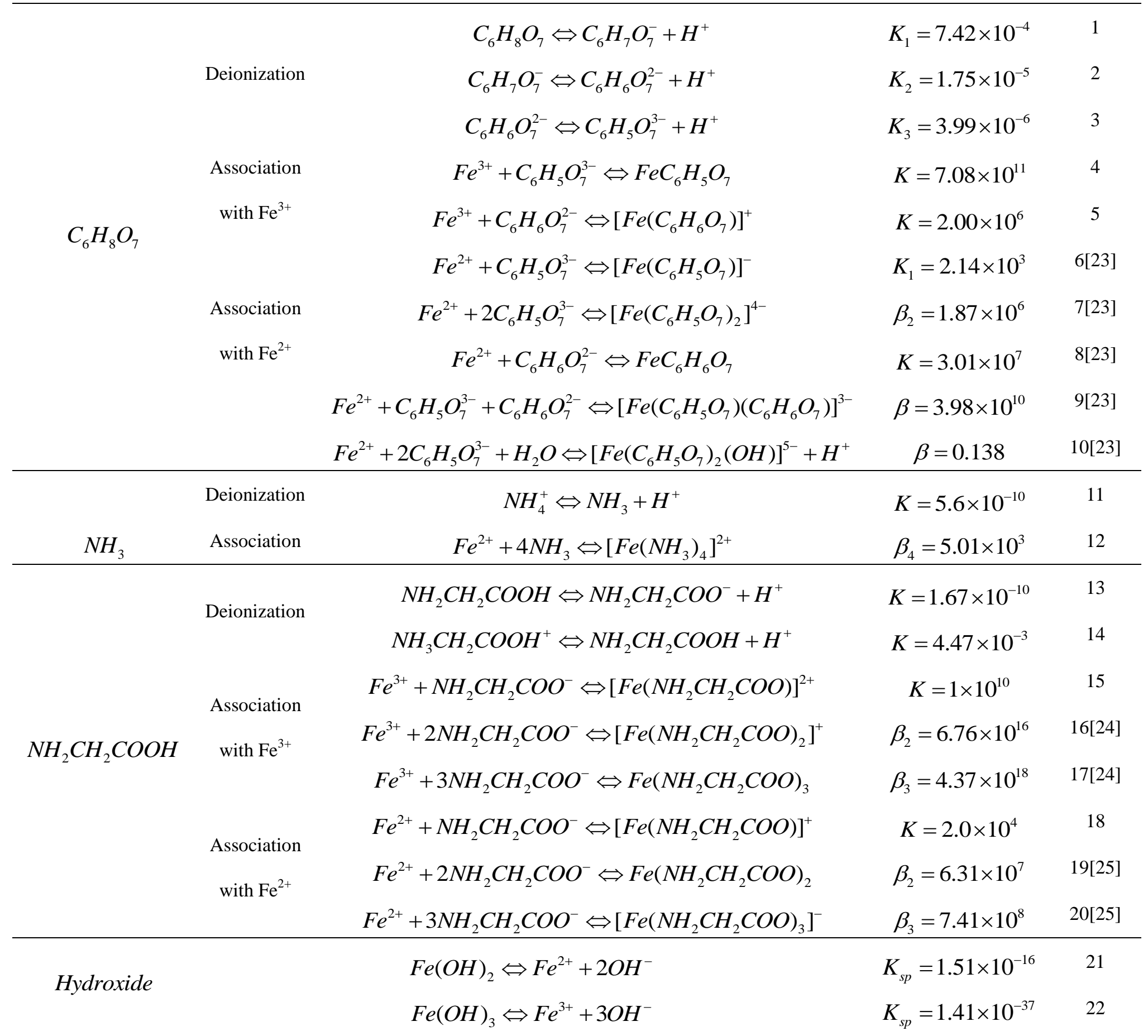




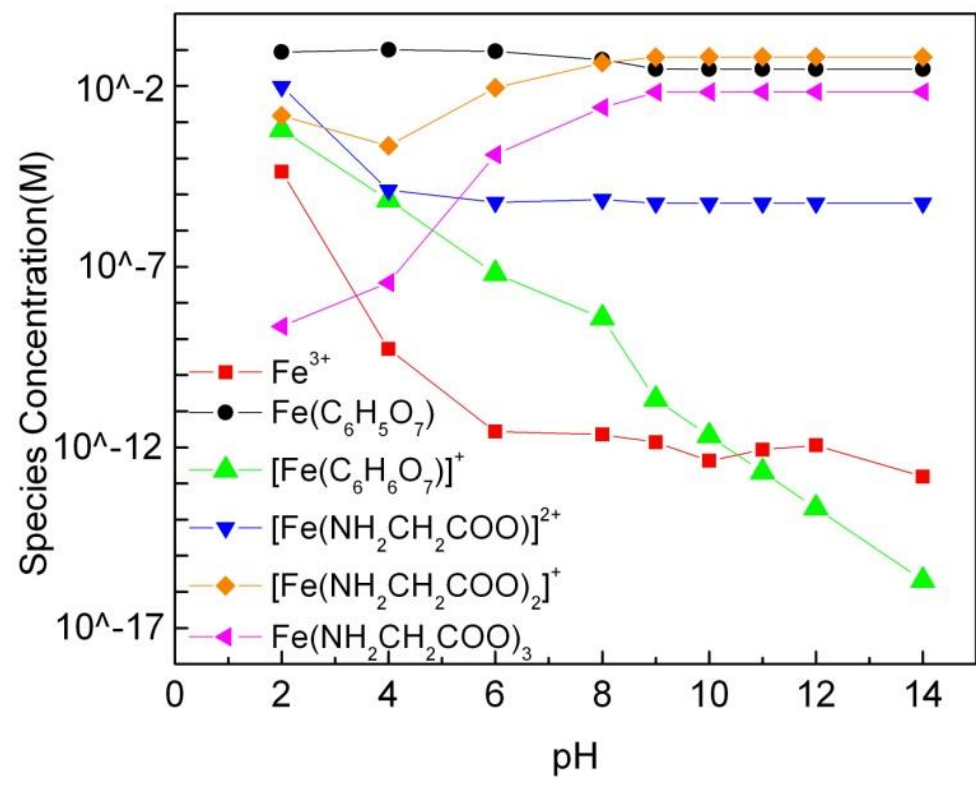

a)

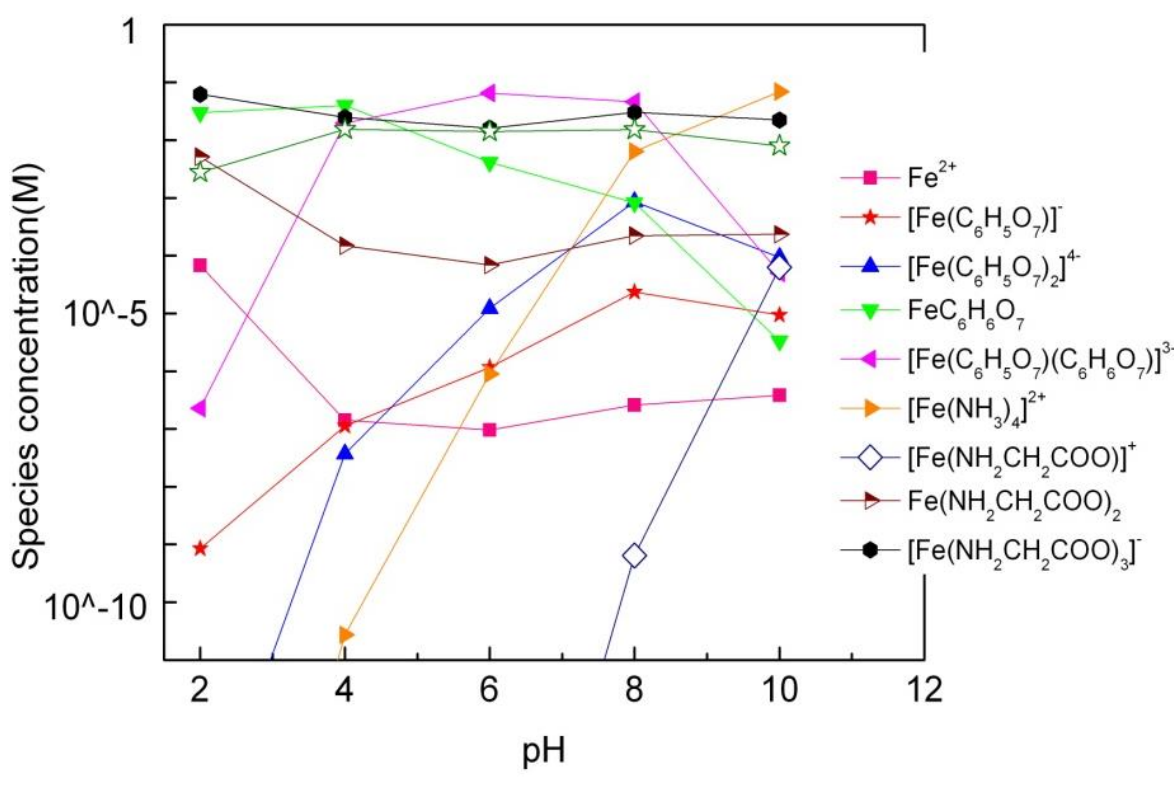

b)

Figure 4.1 Equilibrium concentrations for various Fe species in the solution shown in Table 4.1: a) $\mathrm{Fe}^{3+}$ species; b) $\mathrm{Fe}^{2+}$ species.

The equilibrium concentrations of various $\mathrm{Fe}^{3+}$ and $\mathrm{Fe}^{2+}$ species are shown in Fig.4.1 a) and b) respectively. As shown, the major species for both ferric and ferrous ions are greatly $\mathrm{pH}$ dependent. With respect to the chosen $\mathrm{pH} 8$, the main $\mathrm{Fe}^{3+}$ species are $\mathrm{FeC}_{6} \mathrm{H}_{5} \mathrm{O}_{7} \quad(0.053 \mathrm{M}) \quad$ and $\quad \mathrm{Fe}\left(\mathrm{NH}_{2} \mathrm{CH}_{2} \mathrm{COO}\right)_{3} \quad(0.043 \mathrm{M}), \quad$ and $\left[\mathrm{Fe}\left(\mathrm{C}_{6} \mathrm{H}_{5} \mathrm{O}_{7}\right)\left(\mathrm{C}_{6} \mathrm{H}_{6} \mathrm{O}_{7}\right)\right]^{3-}(0.046 \mathrm{M})$ and $\mathrm{Fe}\left(\mathrm{NH}_{2} \mathrm{CH}_{2} \mathrm{COO}\right)_{2} \quad(0.03 \mathrm{M})$ for $\mathrm{Fe}^{2+}$, while all other species are below 0.01M. Both free $\mathrm{Fe}^{3+}$ and $\mathrm{Fe}^{2+}$ concentrations decrease with $\mathrm{pH}$ and reach a plateau at around $\mathrm{pH} 8$; particularly, the free $\mathrm{Fe}^{3+}$ and 
$\mathrm{Fe}^{2+}$ concentrations at $\mathrm{pH} 8$ are $2.2 \times 10^{-12} \mathrm{M}$ and $2.6 \times 10^{-7} \mathrm{M}$, respectively, both of which are below the solubility limits of $\mathrm{Fe}^{3+}\left(10^{-10} \mathrm{M}\right)$ and $\mathrm{Fe}^{2+}\left(10^{-4} \mathrm{M}\right)$, and therefore, do not form precipitates.

The free $\mathrm{Fe}^{3+}$ and $\mathrm{Fe}^{2+}$ concentrations with only citrate, only glycine and both citrate and glycine are shown in Fig.4.2. For both $\mathrm{Fe}^{3+}$ and $\mathrm{Fe}^{2+}$, citrate is more effective in complexing, which may be due to the fact that citrate has more functional groups than glycine. The combination of citrate and glycine further reduces the concentrations of free $\mathrm{Fe}^{3+}$ and $\mathrm{Fe}^{2+}$, the latter being more evident.

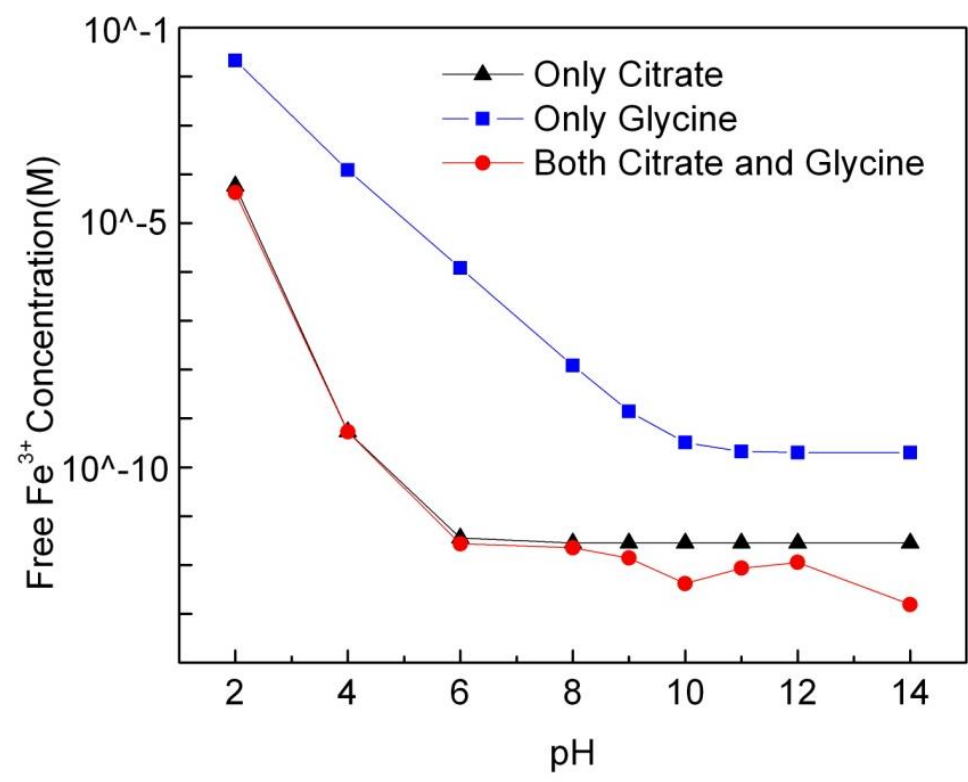

a)

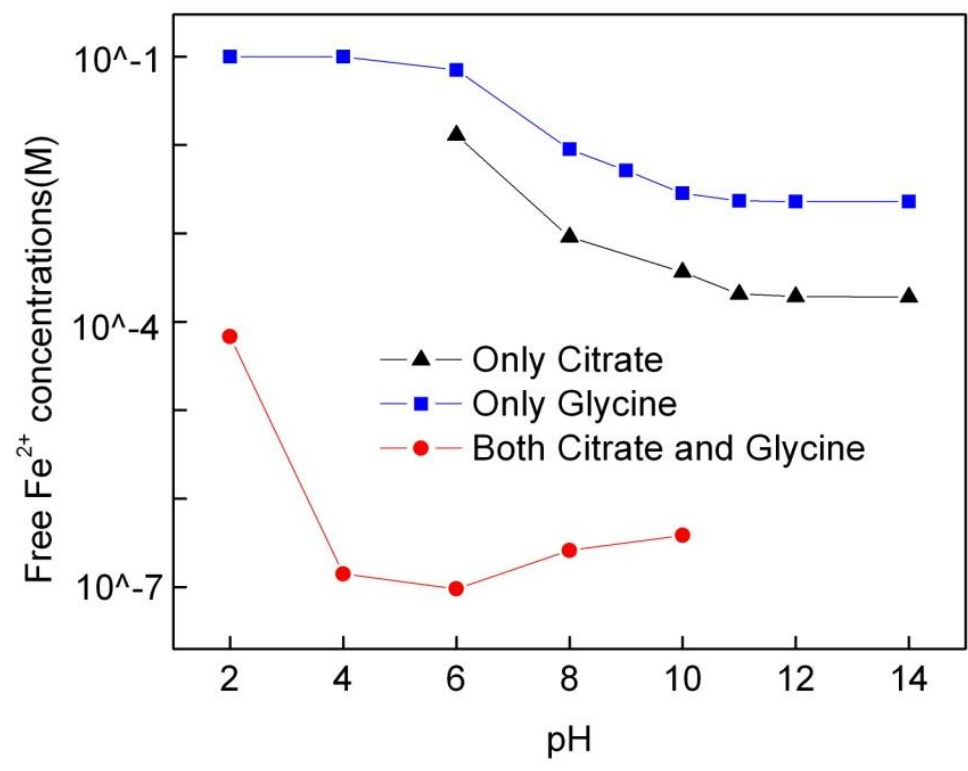

b)

Figure 4.2 Comparison of citrate, glycine and their combination in complexing: a) $\mathrm{Fe}^{3+}$; b) $\mathrm{Fe}^{2+}$. 


\subsubsection{Electrochemical study on Fe-Pt from the alkaline solution}

Displayed in Fig.4.3. is the $\mathrm{CV}$ at a $\mathrm{Au}$ resonator electrode for the background solution only. A small current increase was observed from $-0.6 \mathrm{~V}$ to until $-1.21 \mathrm{~V}$, followed by the rapid increase characterized the onset of hydrogen evolution reaction (HER); for comparison, the calculated equilibrium potential for HER for $\mathrm{pH} 8$ solutions is $-1.08 \mathrm{~V}$.

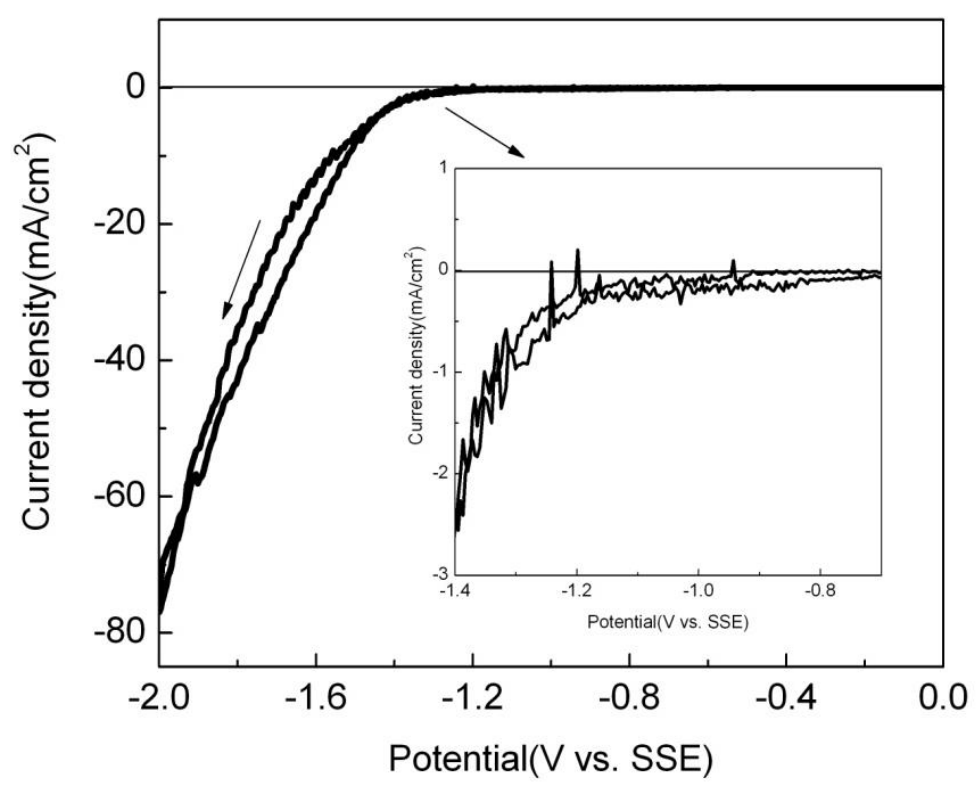

Figure $4.3 \mathrm{CV}$ for the background solution, scan rate $20 \mathrm{mV} / \mathrm{s}$.

The $\mathrm{CV}$ and corresponding rate of mass change $\mathrm{dm} / \mathrm{dt}$ for the background solution with the addition of $15 \mathrm{mM}$ Pt $\mathrm{p}$ salt are shown in Fig.4.4 a). A small current of $\sim-0.2 \mathrm{~mA} / \mathrm{cm}^{2}$ was observed from $-0.6 \mathrm{~V}$ to $-0.9 \mathrm{~V}$, and a monotonic mass increase was observed in the same potential range. Between $-0.9 \mathrm{~V}$ and $-1.1 \mathrm{~V}$, a plateau of $-2 \mathrm{~mA} / \mathrm{cm}^{2}$ was observed, followed by a rapid current increase at $-1.1 \mathrm{~V}$ due to HER. The HER onset is depolarized by $100 \mathrm{mV}$ compared with the background solution, the result of the catalytic effect of Pt towards HER. At higher overpotentials, the vigorous hydrogen evolution interferes with the current measurement, while the mass change shows a hysteretic behavior, with smaller deposition rate at corresponding potential in the return sweep. The current efficiency drops dramatically from $70 \%$ at $-0.9 \mathrm{~V}$ to just several percentages at $-1.5 \mathrm{~V}$, in parallel with HER. This confirms the necessity of an 
alkaline $\mathrm{pH}$ for reasonable deposition rate with $\mathrm{p}$ salt.

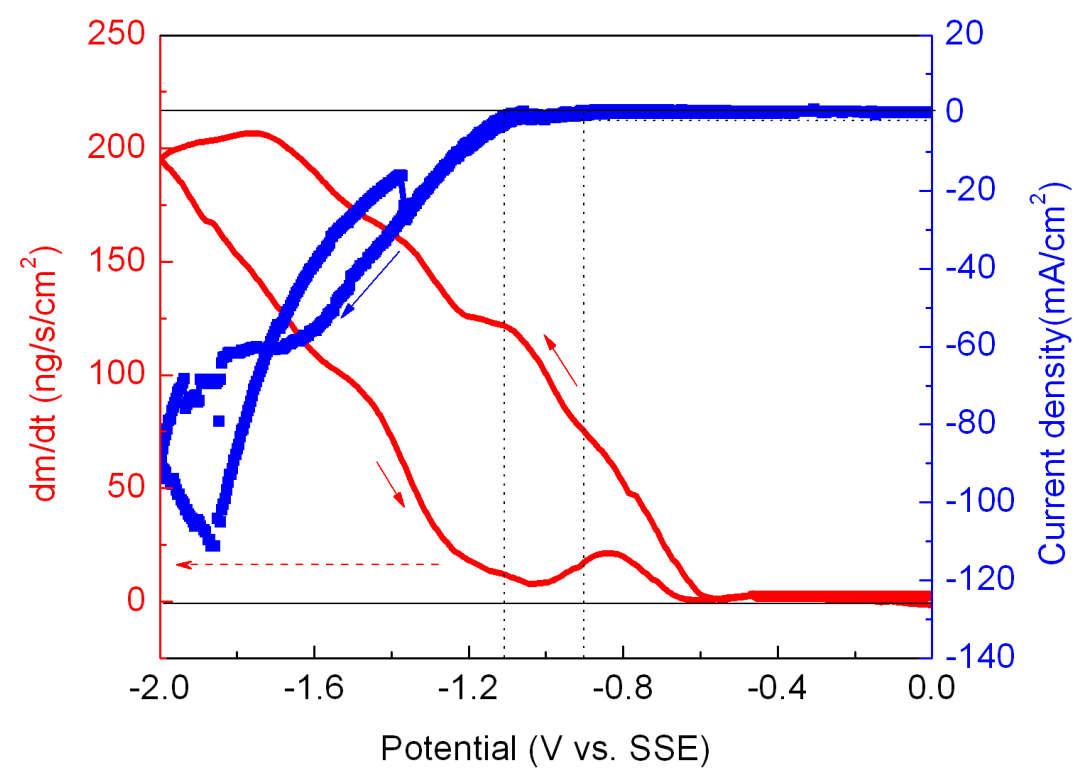

a)

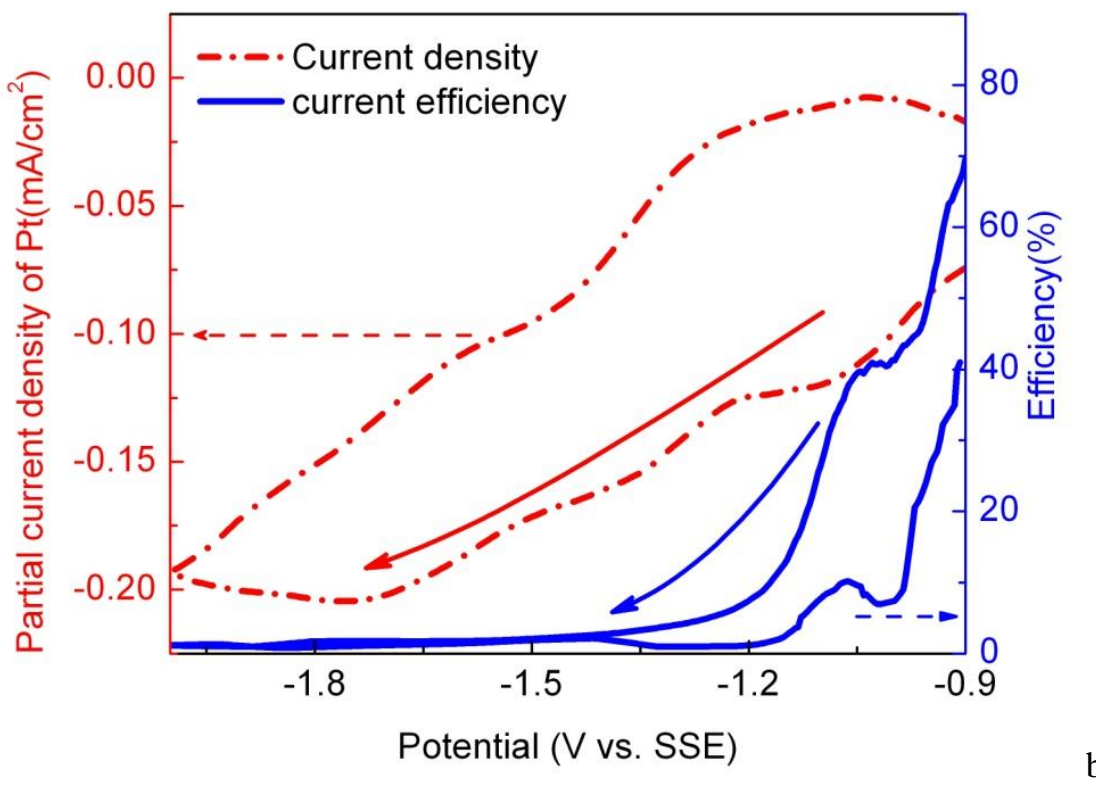

b)

Figure 4.4 a) $\mathrm{CV}$ and rate of mass change for background solution with Pt p salt; b) corresponding partial current for Pt and current efficiency.

$\mathrm{CVs}$ were recorded on $\mathrm{Ru}$ substrates in order to compare the effect of Pt $\mathrm{p}$ salt with respect to the usual acidic Pt chloride solution, as reported in Fig.4.5. Onset of Pt chloride reduction was observed at $-0.2 \mathrm{~V}$, with the diffusion peak at $-0.75 \mathrm{~V}$; the onset of $\mathrm{Pt}$ reduction from the p salt was polarized by $\sim 600 \mathrm{mV}$. It is clear that complexation of Pt by the p salt is much stronger than that in Pt chloride. Meanwhile, no diffusion limited feature was observed for Pt in mass change data for $\mathrm{p}$ salt for the potential 
range used for alloy deposition $(-1.1 \mathrm{~V}$ to $-1.65 \mathrm{~V}$ as will be shown), at variance with the behavior reported for acidic Pt chloride solution [5,6]. This may favor smoother morphology in alloy films.

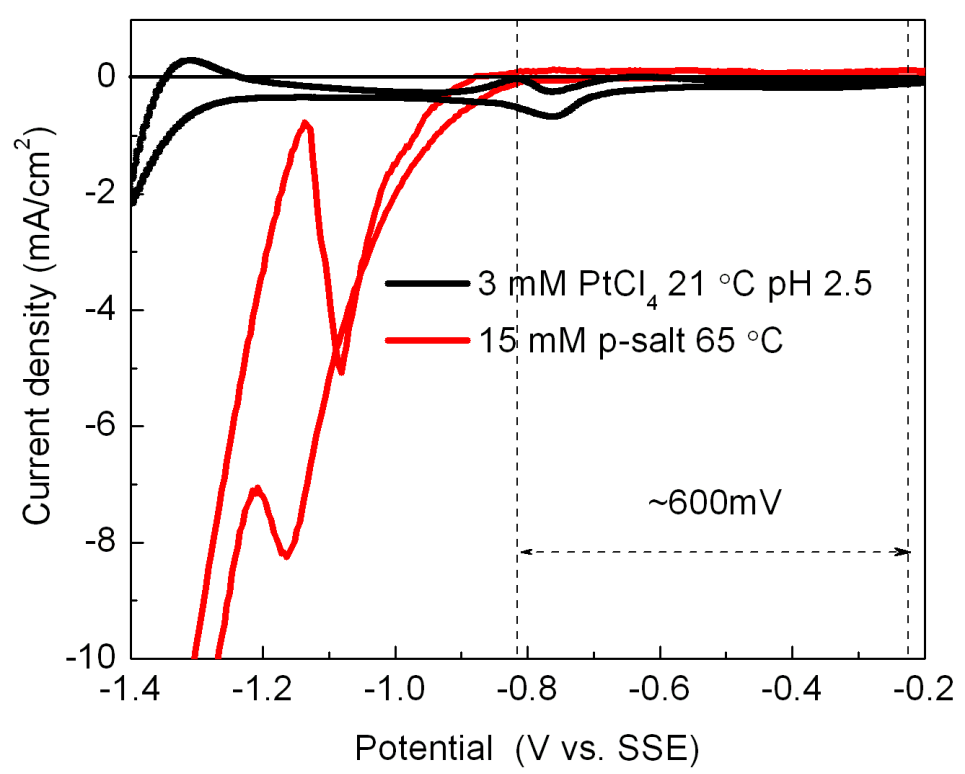

Figure 4.5 CVs comparing the deposition of $\mathrm{Pt}$ on $\mathrm{Ru}$ substrates from an acidic solution containing $3 \mathrm{mM} \mathrm{PtCl}_{4}+0.5 \mathrm{M} \mathrm{NaCl}$ at $\mathrm{pH} 2.5$, and $15 \mathrm{mM} \mathrm{Pt} \mathrm{p}$ salt +the background solution at $\mathrm{pH} 8,65^{\circ} \mathrm{C}$. Scan rate of $20 \mathrm{mV} / \mathrm{s}$.

EQCM data for the background solution with $100 \mathrm{mM} \mathrm{Fe}^{3+}$ are reported in Fig.4.6. In the forward scan, a slow current increase first occured from $-0.5 \mathrm{~V}$ to $-1.05 \mathrm{~V}$, where a plateau current of $-9 \mathrm{~mA} / \mathrm{cm}^{2}$ was reached. This plateau was not observed in the background only solution; on the other hand, there was no mass increase in the corresponding potential range in mass change curve. As a result, this current may be attributed to the one electron transferd reaction of $\mathrm{Fe}^{3+}$ to $\mathrm{Fe}^{2+} . \mathrm{Fe}^{2+}$ is generated from this reaction, therefore its equilibrium potential is shifted cathodically according to Nernst' equation. Assuming a free $\mathrm{Fe}^{2+}$ concentration such that $\frac{\left[\mathrm{Fe}^{2+}\right]}{\left[\mathrm{Fe}^{3+}\right]}=0.1-1$, the redox potential for $\mathrm{Fe}^{3+}$ reduction is estimated to be -0.54 to $-0.6 \mathrm{~V}$, in rough agreement with the experimental observation. The reduction of $\mathrm{Fe}^{3+}$ to $\mathrm{Fe}^{2+}$ occured under diffusion limited conditions between $-1.05 \mathrm{~V}$ to $-1.20 \mathrm{~V}$. Assuming a diffusivity for $\mathrm{Fe}^{3+}$ species of $10^{-5} \mathrm{~cm}^{2} / \mathrm{s}$, and a diffusion layer thickness of $0.5 \mathrm{~mm}$, the limiting current is estimated to be $-1.93 \mathrm{~mA} / \mathrm{cm}^{2}$, in rough agreement with the experimental 
result. Rapid current increase was observed after the plateau, however without any mass change from $-1.3 \mathrm{~V}$ to $-1.6 \mathrm{~V}$. At potentials more negative than $-1.6 \mathrm{~V}$, a large mass increase occured, related to bulk Fe deposition. According to the $\mathrm{CV}$ and mass change data, no mass increase was observed before the bulk deposition, at variance with what has been reported for acidic solutions; this is attributed to the oxide/hydroxide formation and precipitation on the electrode surface [26]. This lends evidence to confirm that the citrate/glycine complexing solution is successful in avoiding or reducing those impurities. In the backward scan, Fe dissolution starts at $-1.4 \mathrm{~V}$, resulting in a gap between onset of deposition and dissolution, indicating that Fe deposition in this alkaline solution is irreversible.

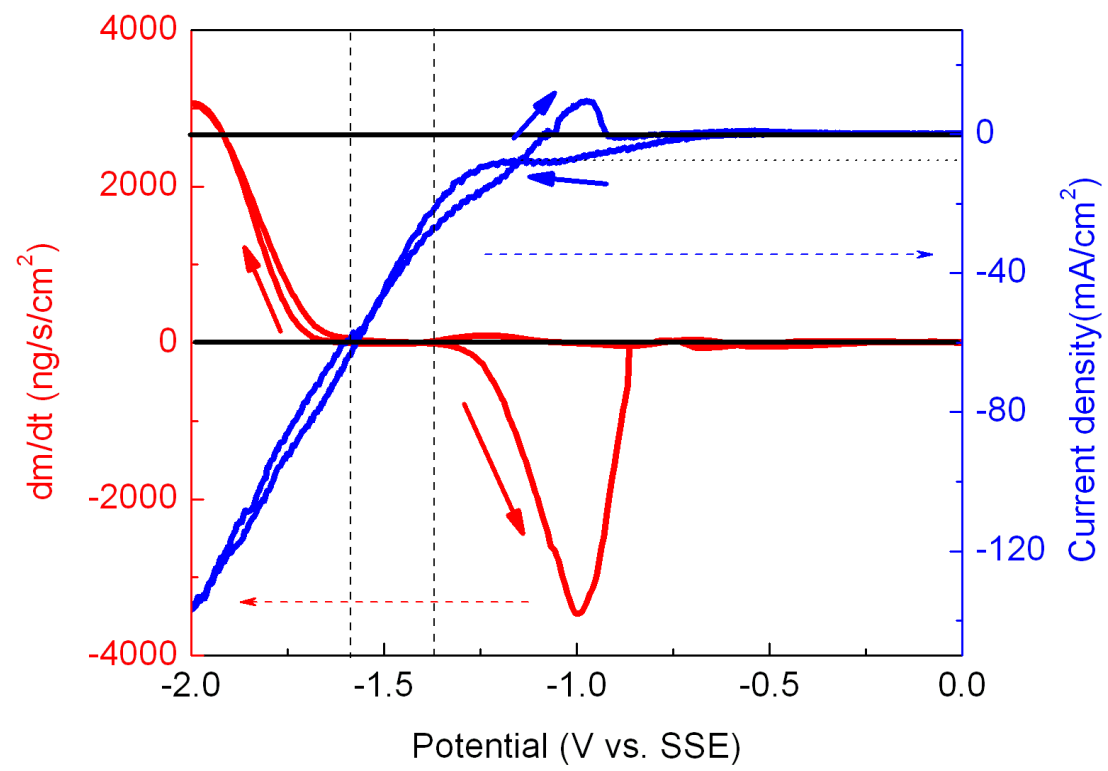

Figure 4.6 CV and rate of mass change for background solution $+100 \mathrm{mM} \mathrm{Fe}^{3+}$ 


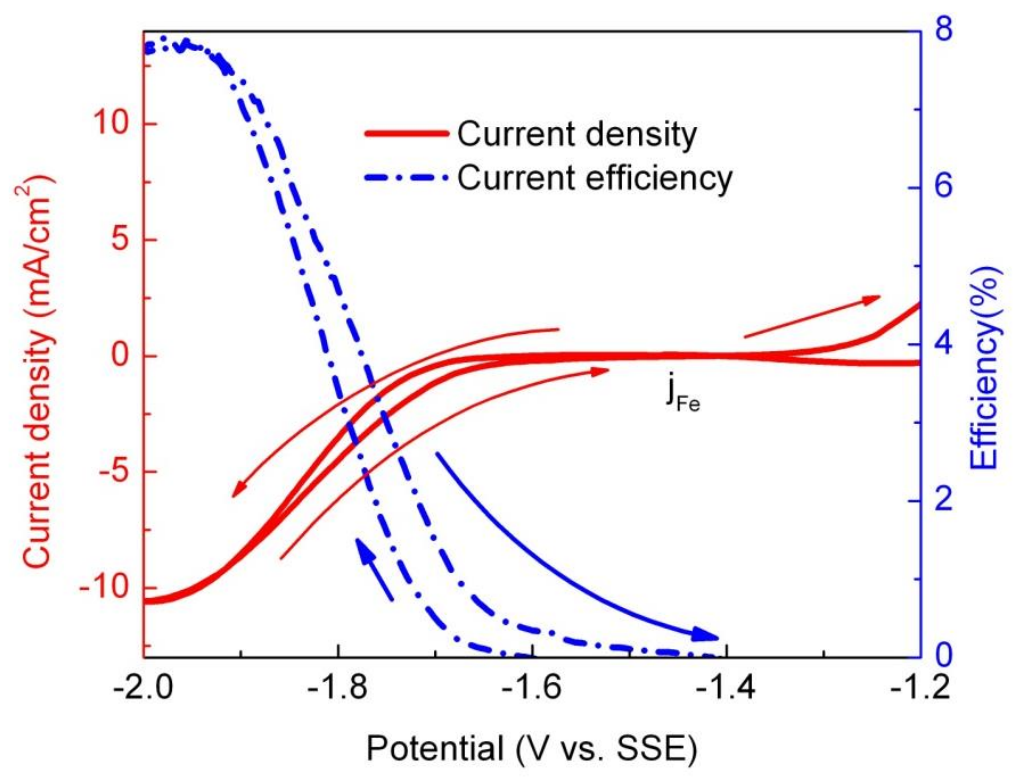

Figure 4.7 Partial current and current efficiency for Fe exacted from Fig 4.6.

The partial current density and the current efficiency for Fe are displayed in Fig.4.7. Current efficiency increases with overpotential but remains less than $13 \%$ due to strong hydrogen generation. The complexation shifts the equilibrium potential cathodically as expected. However, $\mathrm{CV}$ data collected from acidic $\mathrm{Fe}^{2+}$ solution $(0.1 \mathrm{M}$, $\mathrm{pH} 2.5$ ) and the alkaline $\mathrm{Fe}^{3+}$ solution discussed above show that the polarization is about $110 \mathrm{mV}$, much smaller than the shift for Pt deposition from Pt chloride and p salt, suggesting that the alkaline solution helps to bring closer the deposition of Fe and Pt.

$\mathrm{CV}$ and mass change data collected by EQCM for Fe-Pt combined solution are reported in Fig.4.8. From $-0.7 \mathrm{~V}$ to $-1.1 \mathrm{~V}$, a small current and mass increase were observed; compared with the data for Pt only and Fe only solutions, this can be attributed to Pt deposition. From $-1.1 \mathrm{~V}$, however, there was a sharp mass increase, which was not observed for either Pt or Fe only solutions. This is attributed to the onset of Fe co-deposition with $\mathrm{Pt}$, which occured at a potential $\sim 480 \mathrm{mV}$ more positive than pure Fe deposition. This is more clearly shown in the increased/dt curve comparison for Fe only and Fe-Pt solution in Fig.4.9; the shift of Fe deposition in presence of Pt illustrates the UPCD process in Fe-Pt deposition. Indeed, the UPCD behavior is further confirmed by analyzing the composition of films deposited at 
constant potentials. As shown in Fig.4.10, films deposited at $-1.15 \mathrm{~V}$ contain 30at.\% of $\mathrm{Fe}$ in the alloy already, while at $-1.6 \mathrm{~V}$, the potential at which pure Fe starts depositing, 91at.\% of $\mathrm{Fe}$ is detected.

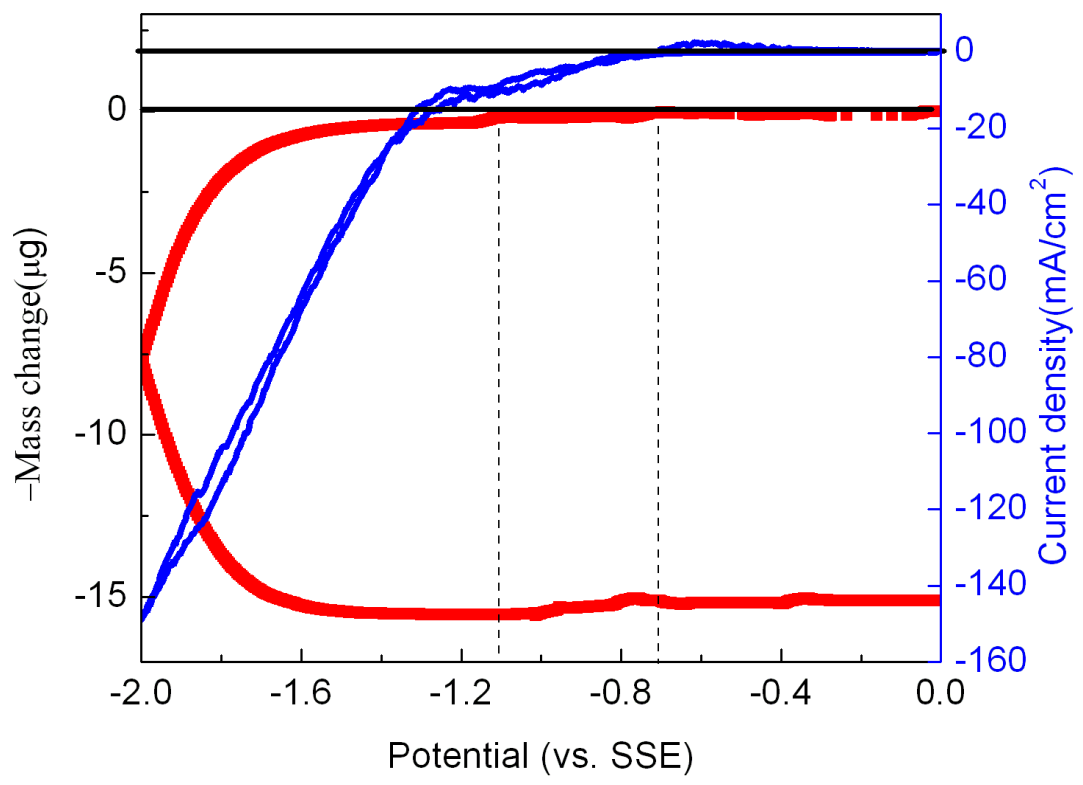

Figure 4.8 CV and mass change data for background solution with addition of $15 \mathrm{mM} \mathrm{Pt} p$ salt and $100 \mathrm{mM} \mathrm{Fe}^{3+}$.

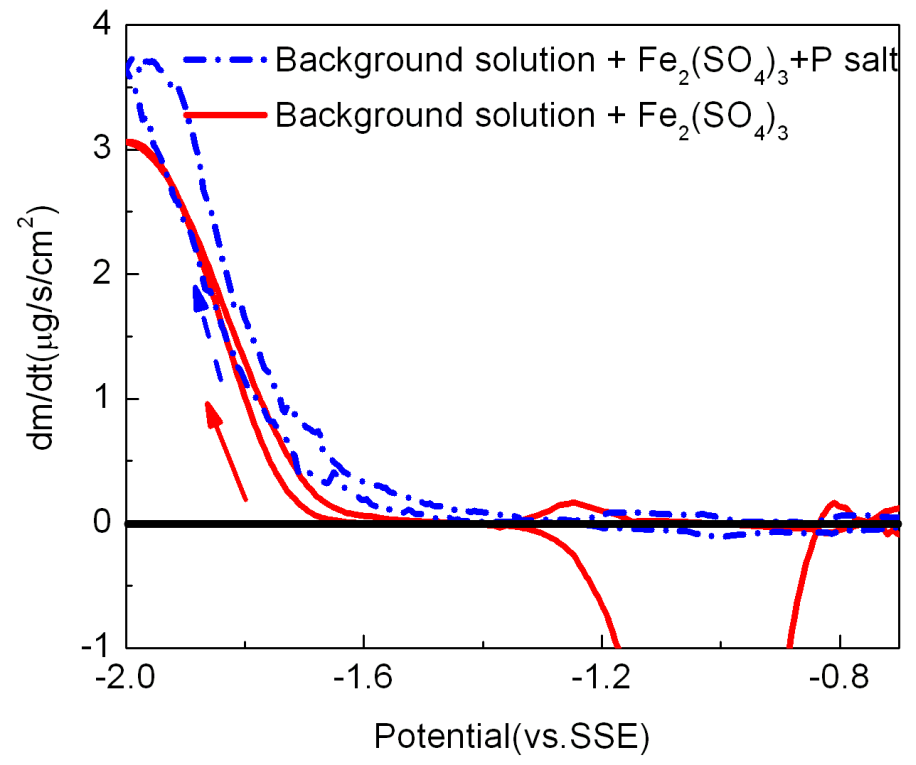

Figure 4.9 Rate of mass change for Fe only and Fe-Pt solution.

The data reported above can be used to propose a simple model for the mechanism of Fe-Pt deposition. At a potential below - $0.6 \mathrm{~V}$, Pt is discharged from the p-salt complex at a rate determined by the applied potential. In parallel, the complexed $\mathrm{Fe}^{3+}$ species 
are reduced to $\mathrm{Fe}^{2+}$ starting around $-0.5 \mathrm{~V}$, and this process reaches the diffusion limiting current at about $-1.1 \mathrm{~V}$. Between -1.1 and $-1.3 \mathrm{~V}$ conditions may be achieved where the interface $\mathrm{pH}$ reaches values sufficient to precipitate hydroxides, as revealed by EQCM data, but this process is limited in extent and does not seem to occur below $-1.4 \mathrm{~V}$. The reduction of $\mathrm{Fe}^{3+}$ complexes results in a high interface concentration of $\mathrm{Fe}^{2+}$ species, which may diffuse away from the electrode, be complexed by citrate and glycine, or be further reduced to Fe. What exactly occurs at the interface depends on the relative rates of the various reactions considered. In any event, the reduction of $\mathrm{Fe}^{3+}$ is diffusion limited over the full range of potentials for which alloy deposition occurs. The diffusion limited current of about $9.5 \mathrm{~mA} / \mathrm{cm}^{2}$ corresponds to a generation rate of $\mathrm{Fe}^{2+}$ of about $98 \mathrm{nmol} / \mathrm{sec} / \mathrm{cm}^{2}$, independent of potential over the range used for alloy deposition. The consumption rate of $\mathrm{Fe}^{2+}$ by deposition at $-1.6 \mathrm{~V}$ can be obtained from the EQCM data in Fig.4.9. The deposition rate at this potential is about $330 \mathrm{ng} / \mathrm{s} / \mathrm{cm}^{2}$. This corresponds to about $4.4 \mathrm{nmol} / \mathrm{s} / \mathrm{cm}^{2}$ of $\mathrm{Fe}^{2+}$ being reduced to $\mathrm{Fe}$ (the alloy is over $90 \% \mathrm{Fe}$ at that potential), which accounts for about $5 \%$ of the $\mathrm{Fe}^{2+}$ generated by reduction of $\mathrm{Fe}^{3+}$. At more positive deposition potentials, where alloys richer in Pt are deposited, this percentage will be much lower. This implies that the steady-state concentration of free $\mathrm{Fe}^{2+}$ at the electrode interface is mainly determined by its diffusion away from the electrode and by changes in its complexation state, and the rate of these processes should be approximately constant over the entire range of deposition potentials. It seems likely, therefore, that $\mathrm{Fe}$ is deposited into the alloy from a $\mathrm{Fe}^{2+}$ species that does not change appreciably in concentration over the entire range of alloy compositions. At low enough deposition rates it is also conceivable that the deposition reaction could occur close to equilibrium between the depositing $\mathrm{Fe}^{2+}$ species and the $\mathrm{Fe}$ in the alloy; this hypothesis is partly supported by the EQCM data for Fe-Pt deposition (Fig.4.9), where at the same current Fe co-deposition occurs with a lower overpotential than Fe elemental deposition, possibly due to a catalytic effect of the Pt present on the surface. Whether this $\mathrm{Fe}^{2+}$ intermediate is a complex or free $\mathrm{Fe}^{2+}$ ion is not known. Whether solution equilibrium is achieved between the various $\mathrm{Fe}$ complexes and free $\mathrm{Fe}^{2+}$ ions at the electrode interface is also not known $a$ 
priori, since diffusion of generated $\mathrm{Fe}^{2+}$ into the solution occurs rapidly and complexation may be slow.

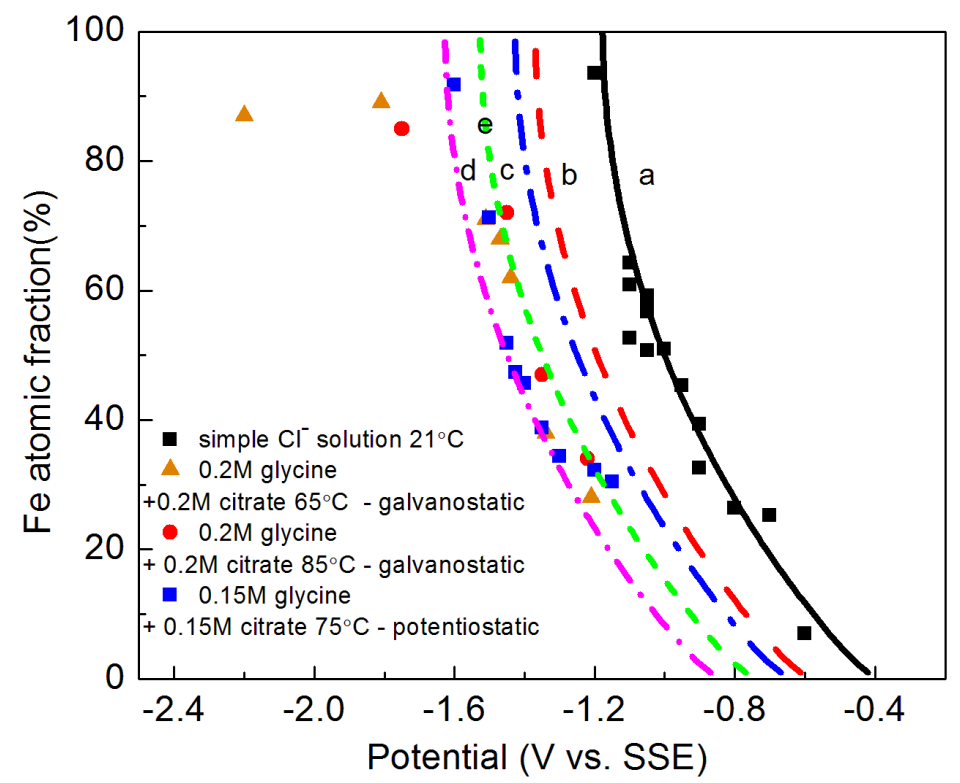

Figure 4.10 Composition vs. potential behavior for Fe-Pt alloys deposited from the alkaline solution.

Oxygen content, as a result of oxide and/or hydroxide formation, in Fe-Pt deposits from acidic solutions have been reported to be up to $30 \mathrm{at}$. $\%$ for the equiatomic alloys $[5,10]$. We have detected the oxygen in our films using EDS with low energy electrons and confirmed using AES. As shown in Fig.4.11, 1 to 6at.\% of oxygen is detected for all alloys from Pt rich to Fe rich, a significant reduction compared with acidic solutions. AES spectra confirm the low oxygen content in our films from p salt solution, however also indicate up to 17 at. $\%$ of carbon incorporated into the deposits. The exact mechanism of the carbon detected is not clear; it may be due to the trapping of citrate and / or glcyine molecule during deposition, or the decomposition of such organics. 


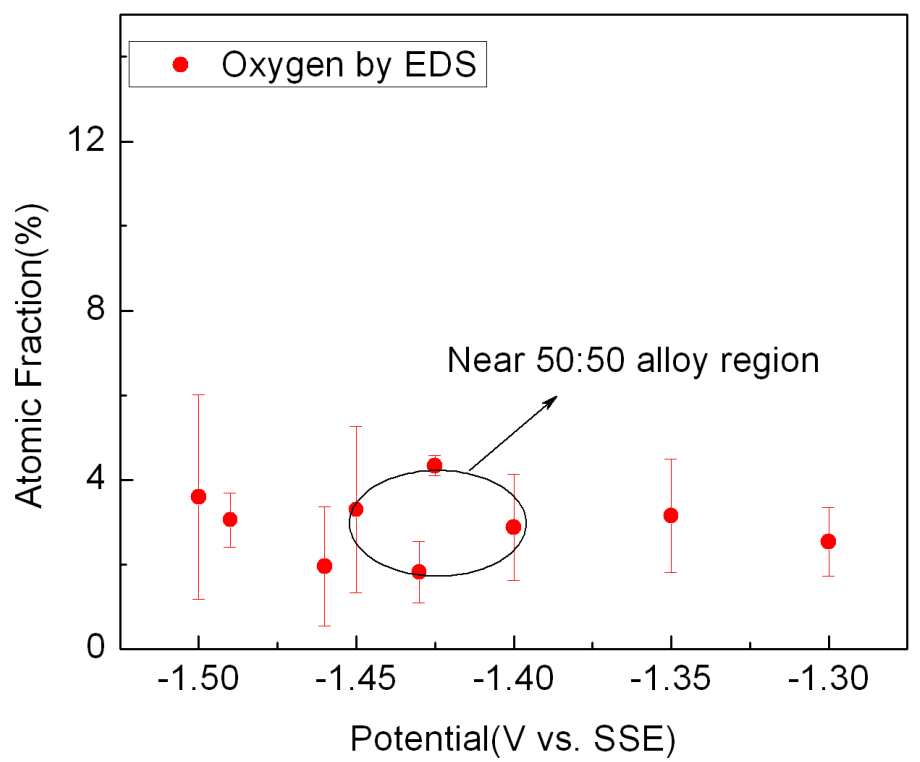

Figure 4.11 Oxygen content in Fe-Pt deposits from the alkaline solutions by EDS using an acceleration voltage of $5 \mathrm{kV}$.

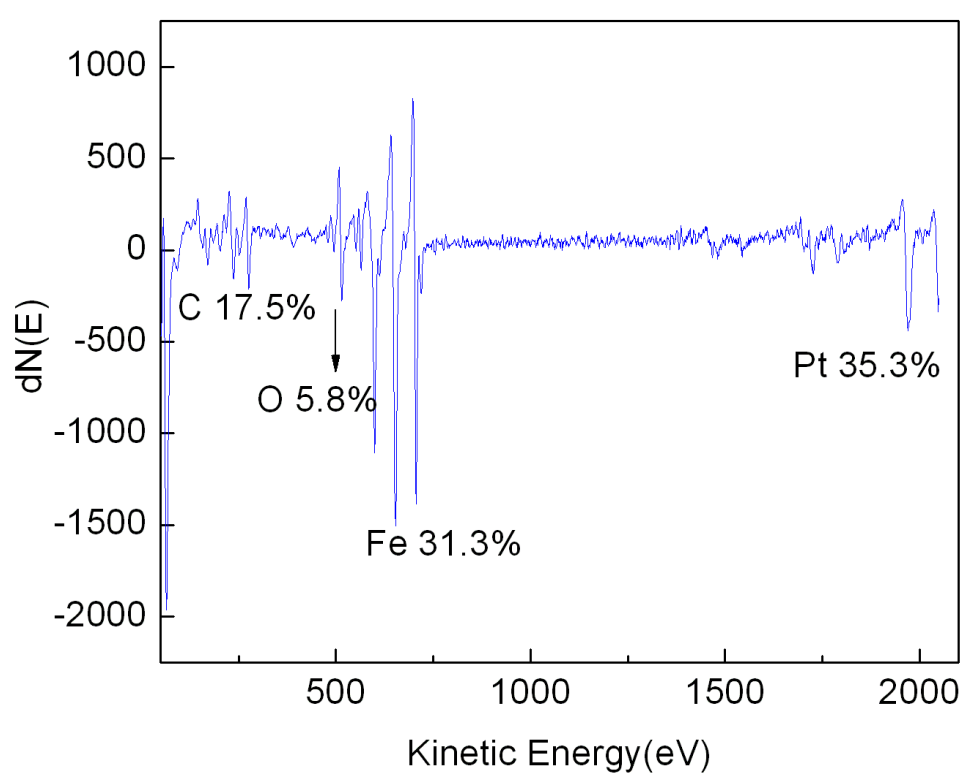

Figure 4.12 AES spectrum confirming the low oxygen content and indicating carbon incorporation.

The depolarization of $\mathrm{Fe}$ reduction during $\mathrm{Fe}-\mathrm{Pt}$ codeposition under conditions approaching thermodynamic equilibrium can be predicted theoretically using an appropriate expression for the enthalpy of mixing of Fe-Pt $[5,27]$ and substituting the resulting Fe activity in the alloy in equation (1.23) to obtain the equilibrium redox potential of $\mathrm{Fe}$ at a Fe-Pt surface. This relationship has been observed to hold for Fe-Pt alloys deposited from acidic solutions [5]. In Fig.4.10 we compare our alloy 
composition vs. potential data obtained at various temperatures $T$ and various complexant concentrations with those of Ref. [5]. While these data have been obtained at different $T$, this comparison is still meaningful. In fact, in the regular solution approximation the enthalpy of mixing does not depend on $T$. $T$ affects only the entropic term in the free energy, which in the range of temperatures investigated remains negligible with respect to the enthalpy. Our results are shifted in the cathodic direction with respect to those reported in Ref. [5], fitted by line (a); we assume the shift to be originated by the decrease in free $\mathrm{Fe}^{2+}$ ion concentration upon complexation; this results in a decrease of $a_{\mathrm{i}}^{\text {ion }}$, leaving $a_{\mathrm{i}}^{\text {alloy }}$ unchanged. Since $a_{\mathrm{i}}^{\text {ion }}$ is independent of alloy composition, the curves will maintain the same shape and will only be shifted in the horizontal direction. Possible fitting curves for our data are also reported in Fig.4.10; these were obtained shifting curve (a) horizontally by a variable amount, calculated under different assumptions. Curve (b) is obtained by assuming the equilibrium potential of $\mathrm{Fe}^{2+}$ at a Fe-Pt electrode $E_{\mathrm{Fe}}{ }^{2+} / \mathrm{Fe}(\mathrm{Fe}-\mathrm{Pt})$ to be determined by the calculated equilibrium concentration of $\mathrm{Fe}^{2+}$ (Fig.4.2(b)). Curve (c), (d), (e) are obtained by taking the $E_{\mathrm{Fe} 2+/ \mathrm{Fe}}(\mathrm{Fe}-\mathrm{Pt}$ ) determined by EQCM experiments (Fig.4.6) and assuming that this is associated respectively to: (c) the onset potential for $\mathrm{Fe}$ dissolution, (d) the potential midway between Fe deposition and dissolution, or (e) the onset potential of $\mathrm{Fe}$ deposition. The actual equilibrium potential for the $\mathrm{Fe}^{2+} / \mathrm{Fe}$ reaction is difficult to determine due to the intrinsic irreversibility of the corresponding deposition process for pure Fe deposition; however, an approximate value can be determined assuming a symmetric Butler-Volmer characteristics for Fe deposition and taking this potential to be the average between the two potentials where the partial current $\boldsymbol{j}_{\mathrm{Fe}}$ assumes predetermined small values $\boldsymbol{j}^{*}$ and $\boldsymbol{j}^{*}$, as done in curve (d). The experimental data are all positioned between the curves constructed under hypotheses (c) and (e), using EQCM data. This lends partial support to the assumption that Fe co-deposition indeed occurs under conditions of near-equilibrium at the interface. Alloy composition can therefore be approximately predicted by an appropriate estimate of the $\mathrm{Fe}^{2+} / \mathrm{Fe}$ redox potential and evaluation of the enthalpy of mixing in the solid state. The discrepancy between the shift in the curves predicted 
from solution equilibria and the experimental data may be the result of uncertainties in the equilibrium constants extracted from the literature; in fact, such values as reported from different sources may vary across various orders of magnitude. Another reason for such discrepancy may be that solution equilibrium is not achieved between the $\mathrm{Fe}^{2+}$ species generated from reduction of $\mathrm{Fe}^{3+}$ and the $\mathrm{Fe}^{2+}$ species (complexed or free) from which deposition occurs. A deviation from the curve trend is however observed in the composition data for the highest Fe fractions, where Fe deposition rate increases and consequently kinetic effects cannot be neglected [28].

The approximate agreement of the experimental data with the thermodynamic prediction is somewhat surprising, for more than one reason. Oxygen incorporation is limited, and its presence should not significantly affect thermodynamic predictions; however, it is expected that the large amount of incorporated carbon may change the energetics of formation of the alloy system. That this does not occur is probably a consequence of the purely physical nature of the incorporation of $\mathrm{C}$ compounds, which may be decomposed through side reactions and could be kinetically trapped by the growing film, without affecting chemical bonding. Most important however is the evidence that $\mathrm{Fe}$ co-deposition might be occurring close to conditions of local equilibrium between the depositing $\mathrm{Fe}^{2+}$ species and the $\mathrm{Fe}$ in the alloy; this is unexpected, particularly in view of the strong complexation of $\mathrm{Fe}$ ions and the intrinsic irreversibility of the reduction process observed for the deposition of pure Fe. However, it is possible that the Fe-Pt alloy surface is more catalytic for the deposition of Fe from these solutions than a pure Fe surface, as suggested by comparison of the EQCM curves for Fe and Fe-Pt deposition. 


\subsection{Morphology, crystalline structure and magnetic properties of single layer Fe-Pt films}

\subsubsection{Morphology of as-deposited alloys}

Films ( 100nm) with various compositions are imaged with SEM (Fig.4.13). For equiatomic and Pt rich compositions, the apparent grain sizes were similar, about 25 $\mathrm{nm}$; for Fe rich films, larger grains were observed, $30-40 \mathrm{~nm}$. A comparison of the morphology between films from our alkaline solutions and that of acidic solutions were made by AFM, as shown in Fig.4.14. Smaller grain size was observed for the alkaline solution, and the root- mean-square roughness decreased from $3.1 \mathrm{~nm}$ to 1.5 $\mathrm{nm}[21]$.
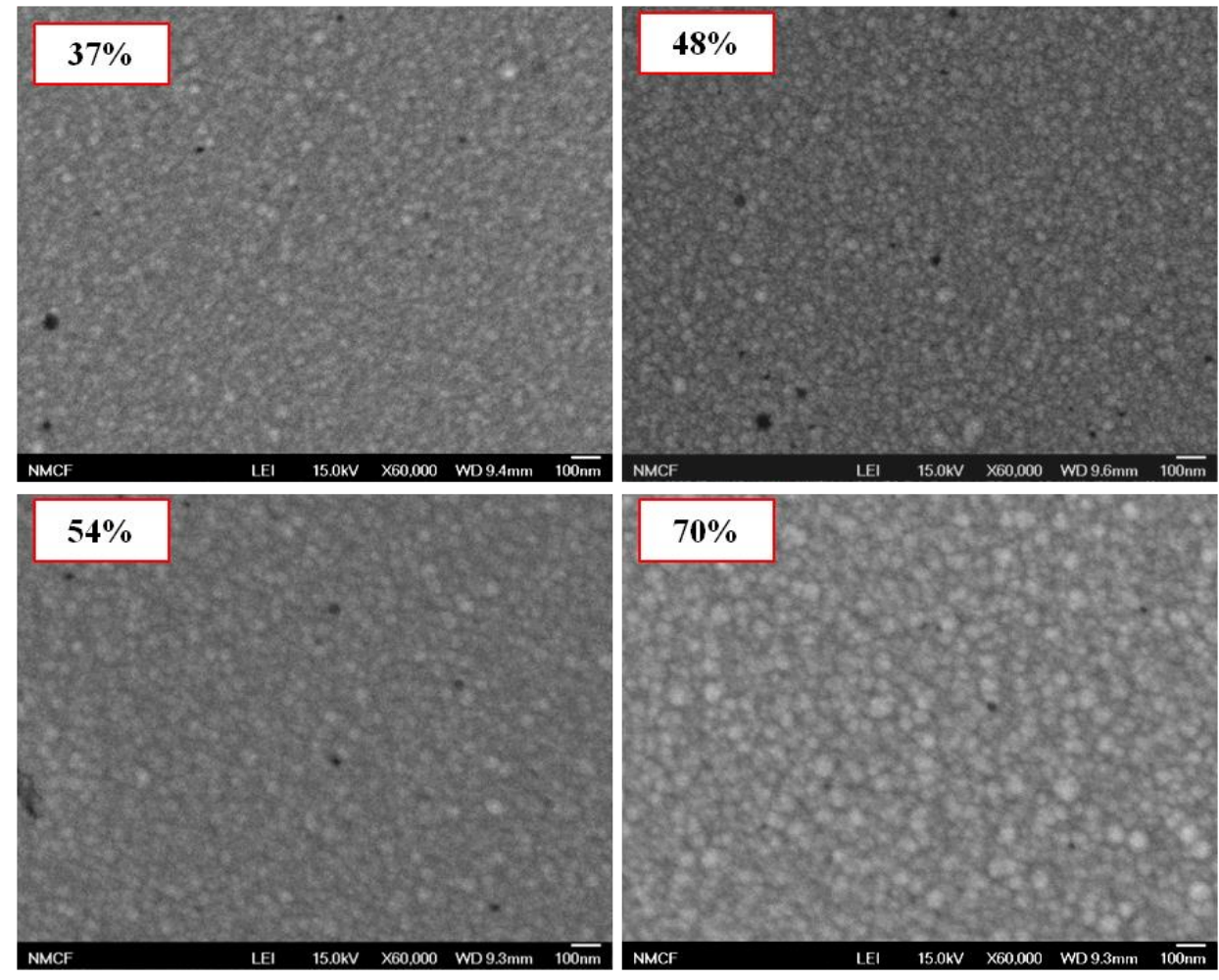

Figure 4.13 SEM images for as-deposited films $(\sim 100 \mathrm{~nm})$ with various Fe fractions. 

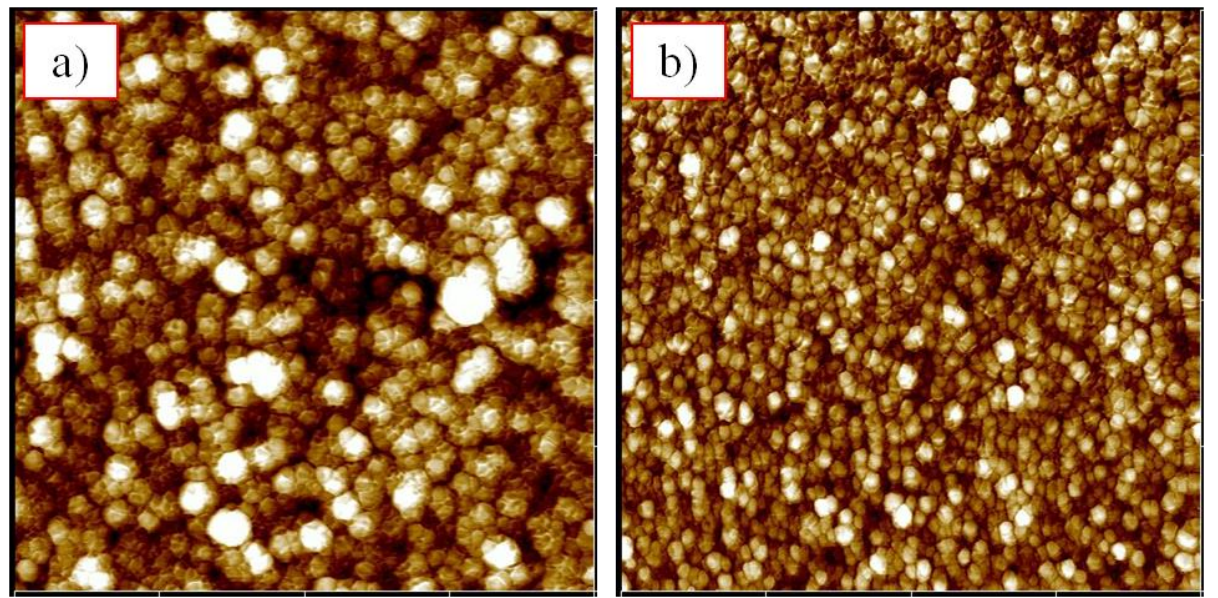

Figure 4.14 AFM images for two near equiatomic films $(\sim 100 \mathrm{~nm})$ for: a) $3 \mathrm{mM} \mathrm{PtCl}_{4}+0.11 \mathrm{M}$ $\mathrm{FeCl}_{2}+0.5 \mathrm{M} \mathrm{NaCl}, \mathrm{pH} 2.5,21^{\circ}$; and b) the alkaline solution. $1 \mu \mathrm{m} \times 1 \mu \mathrm{m}$ area.

However, AFM is not capable of detecting features that are smaller than the tip radius of curvature. Due to this limitation, Hydrogen UPD (HUPD) has also been used to determine the roughness factors $(\mathrm{RF}=$ real area/geometrical area) for $\mathrm{Pt}$ and $\mathrm{Fe}-\mathrm{Pt}$ films deposited from the two solutions discussed above, as shown in Fig.4.15. The solution used for $\mathrm{CV}$ s was $1 \mathrm{M} \mathrm{H}_{2} \mathrm{SO}_{4}$, and the HUPD charge was integrated from -0.4 to $-0.66 \mathrm{~V}$. The RF was estimated by taking the ratio of the integrated charge to the theoretical value of HUPD on polycrystalline Pt $\left(210 \mu \mathrm{C} / \mathrm{cm}^{2}\right)$. Deposition under kinetic activation condition would favor smoother and more compact morphology. This is confirmed by the direct comparison of Pt films from the chloride solution and the alkaline solution, showing RF of 5.1 and 1.2, respectively. The direct HUPD for Fe-Pt films cannot be obtained since dealloying of Fe-Pt during the measurement occurs, but the measurement may still lend some support for the more compact structure of the film from alkaline solution than that of the Pt chloride solution. 


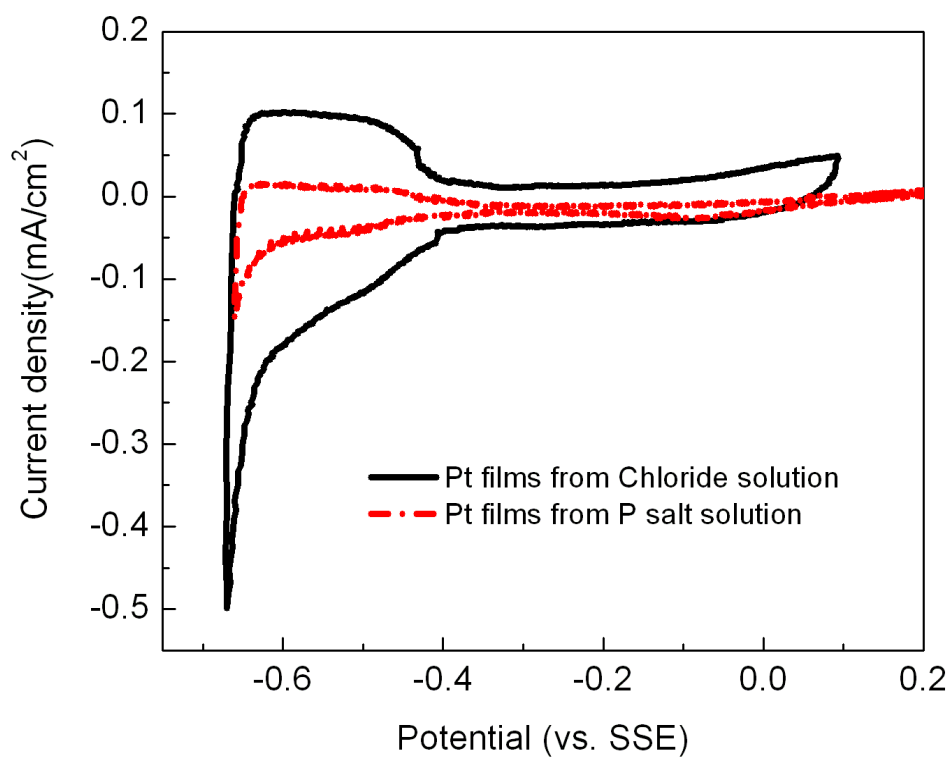

a)

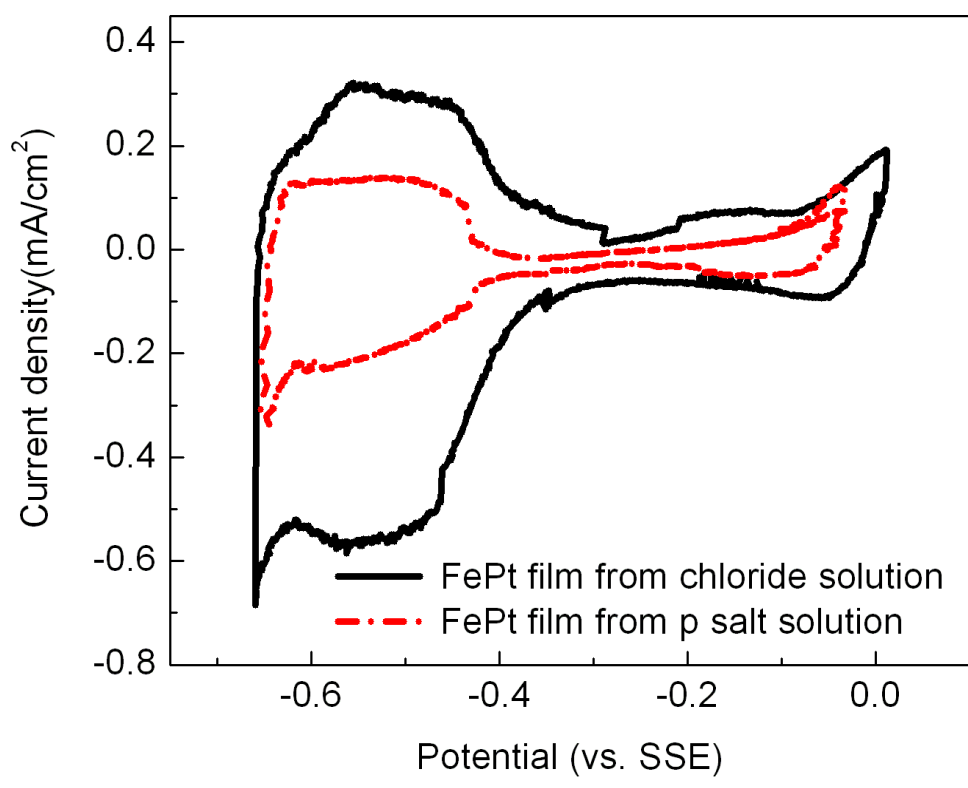

b)

Figure 4.15 CVs for a) Pt films ( $50 \mathrm{~nm}$ ) from chloride solution and the alkaline solution (RF 5.1vs. 1.2); b) Fe-Pt films (near equiatomic, $\sim 100 \mathrm{~nm}$ ) from chloride solution and the alkaline solution (RF 22vs. 10.1); data are for the second scan of CV.

\subsubsection{Crystalline structure and magnetic properties}

The crystalline structures of the as-deposited films $(\sim 100 \mathrm{~nm})$ are determined using XRD (Figure 4.16) [29]. For Pt rich films, a face centered cubic (FCC) structure was observed; the FCC (111) peak shifted to higher angle with increasing Fe fraction, indicating the formation of a series of solid solutions. For Fe rich films ( $>56$ at.\%), a 
body centered cubic (BCC) structure was detected, with the peak (110) close to that of Fe BCC (110) peak. The lattice constants for the alloys with various compositions were calculated by fitting the alloy (111) peak profile and compared with bulk alloy data extracted from literature [30]. Despite slightly smaller values, they agree well the bulk alloy data (Fig.4.17).

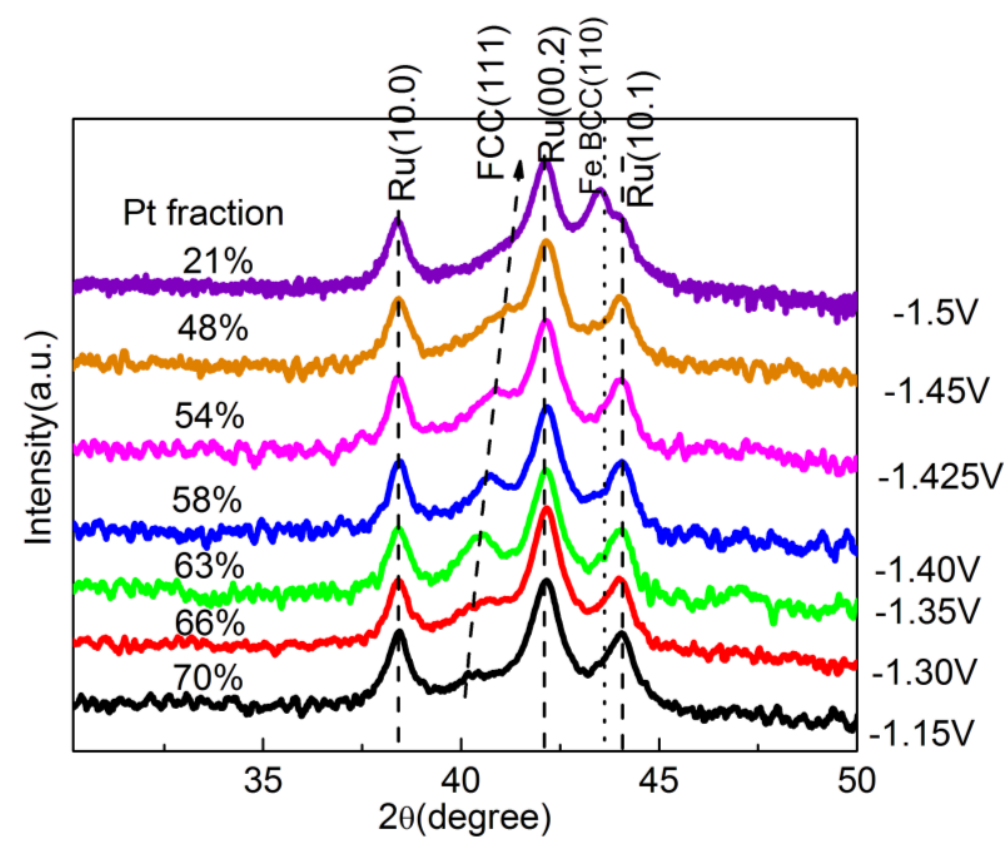

Figure 4.16 XRD patterns for as-deposited Fe-Pt films ( 100 nm).

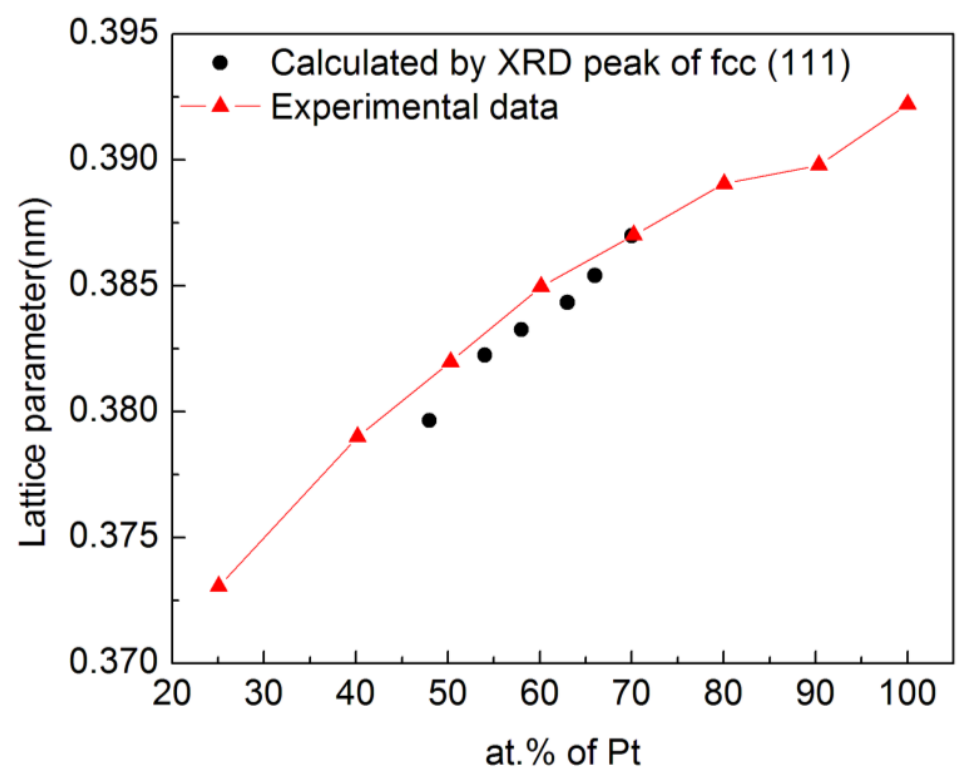

Figure 4.17 Lattice constant as a function of Fe fraction for the as-deposited Fe-Pt films ( 100 nm) calculated by fitting the FCC (111) peak in Fig.4.16. 


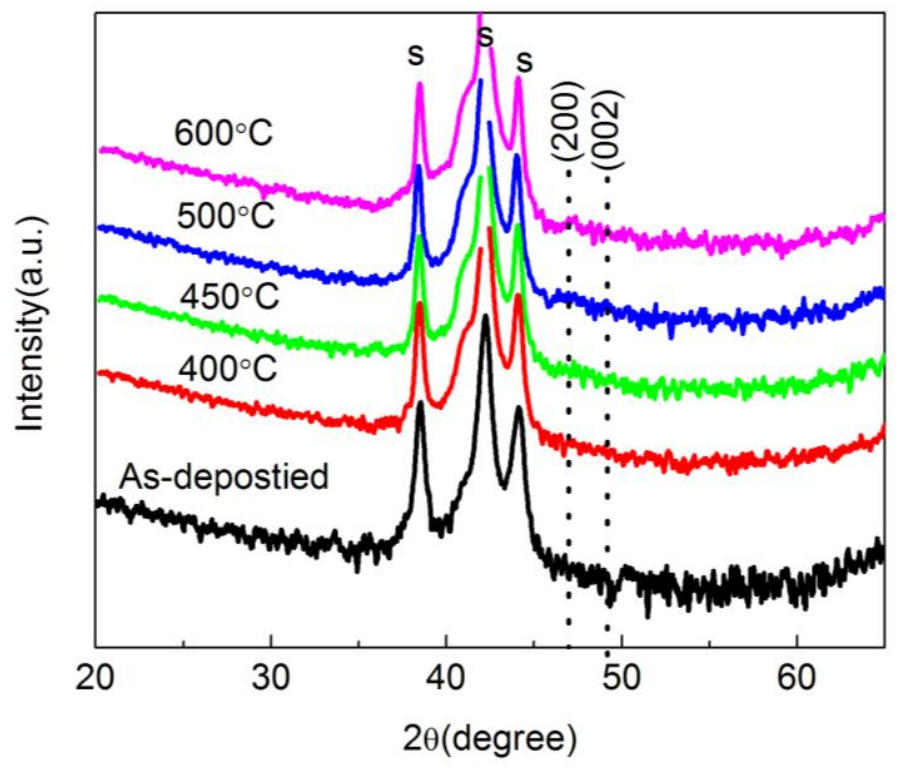

a)

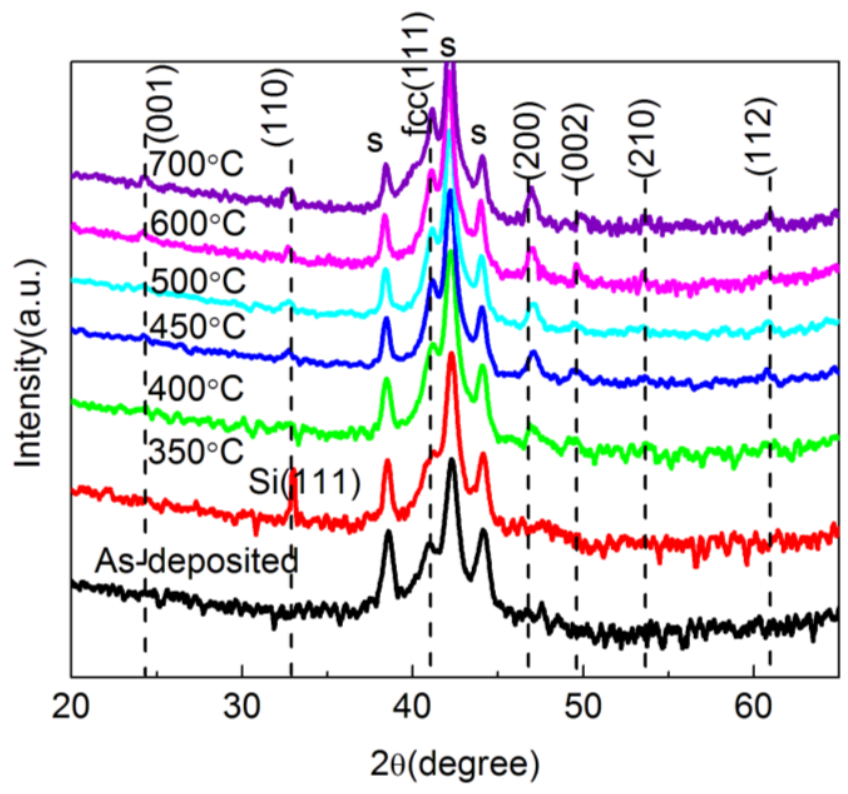

b)

Figure 4.18 XRD patterns for films of different thickness as-deposited and after annealing for $1 \mathrm{~h}$ under flowing forming gas at various temperatures: a) $30 \mathrm{~nm}$; b) $93 \mathrm{~nm}$; deposited on $\mathrm{Ru}$; Si peak is from the wafer.

Similar to the films deposited from acidic solutions, or by other deposition techniques at or close to room temperature, the as-deposited films are magnetically soft, showing coercivity of less than 100 Oe, consistent with the random FCC phase from XRD. In order to study the magnetic hardening mechanism in these alloys, near equiatomic films with thickness ranging from $13 \mathrm{~nm}$ to $93 \mathrm{~nm}$ were annealed under flowing forming gas for $1 \mathrm{~h}$. Upon annealing, for films with thickness less $30 \mathrm{~nm}$, XRD patterns showed only an increase in intensity of the (111) peak, probably due to grain 
growth; for the 30nm films, however, besides the intensity increase of (111) peak, a faint splitting of (200) into (200) and (002) is observed at relatively high temperature $\left(\sim 600^{\circ} \mathrm{C}\right)$, but the other superlattice peaks, which characterizes the appearance of the $\mathrm{L1}_{0}$ phase were not evidently shown. For the thicker films (93nm), the (200) separation was already clearly presented at $350^{\circ} \mathrm{C}$, and after annealing at $450^{\circ} \mathrm{C}$, the other superlattice peaks, such as (001) and (110) were clearly observed. After annealing at higher temperatures, no appreciable intensity increase for these peaks was observed, suggesting that full transformation may have already completed at $450^{\circ} \mathrm{C}$.

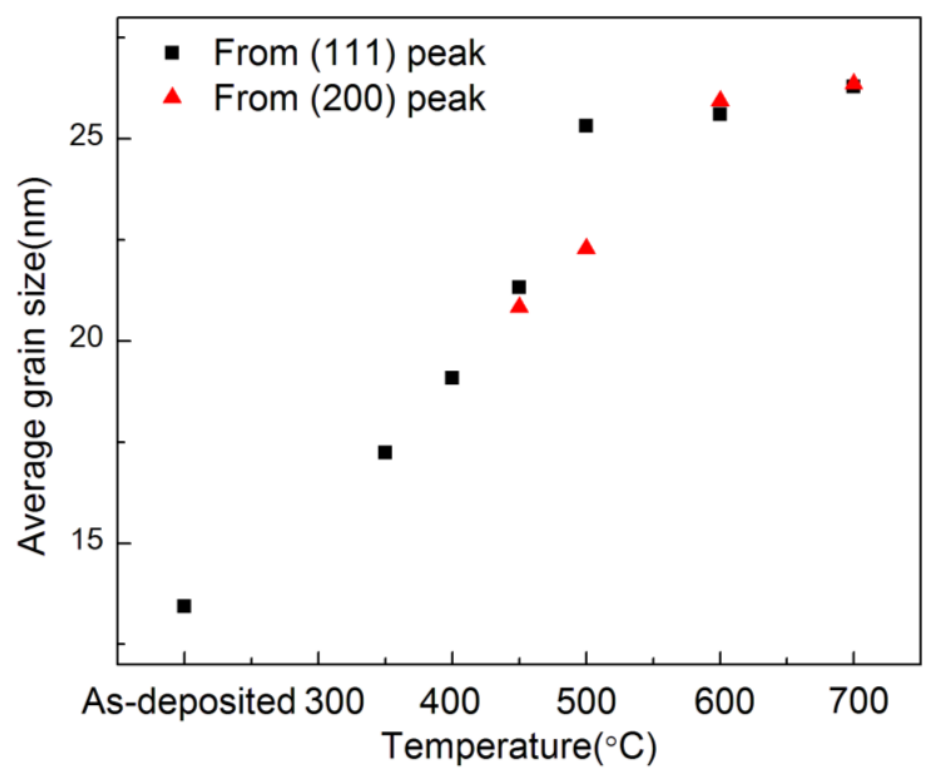

Figure 4.19 Coherent lengths as a function of annealing temperatures for the $93 \mathrm{~nm}$ film by fitting the XRD patterns in Fig.4.18 b).

The Scherrer equation [31] was applied to estimate the coherence lengths in the direction of perpendicular to the films by fitting the (111) or (200) peak in the XRD patterns of the $93 \mathrm{~nm}$ film, neglecting instrumental and stress effect on the peak width. As shown in Fig.4.19, the as-deposited film showed a grain size of $12 \mathrm{~nm}$; grain size kept increasing upon annealing until $500^{\circ} \mathrm{C}$ to about $25 \mathrm{~nm}$, and it remained approximately constant upon further annealing. 


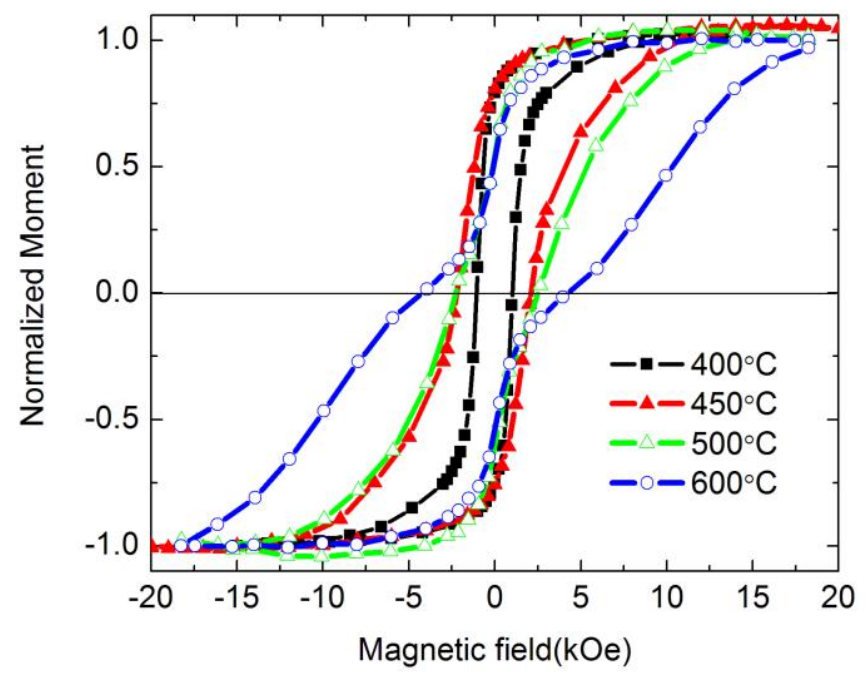

a)

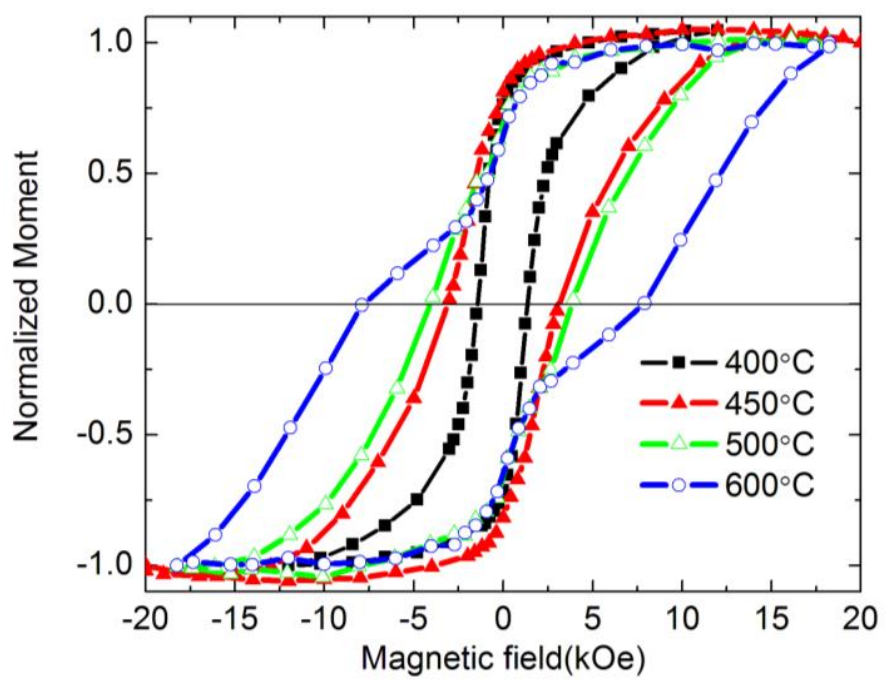

b)

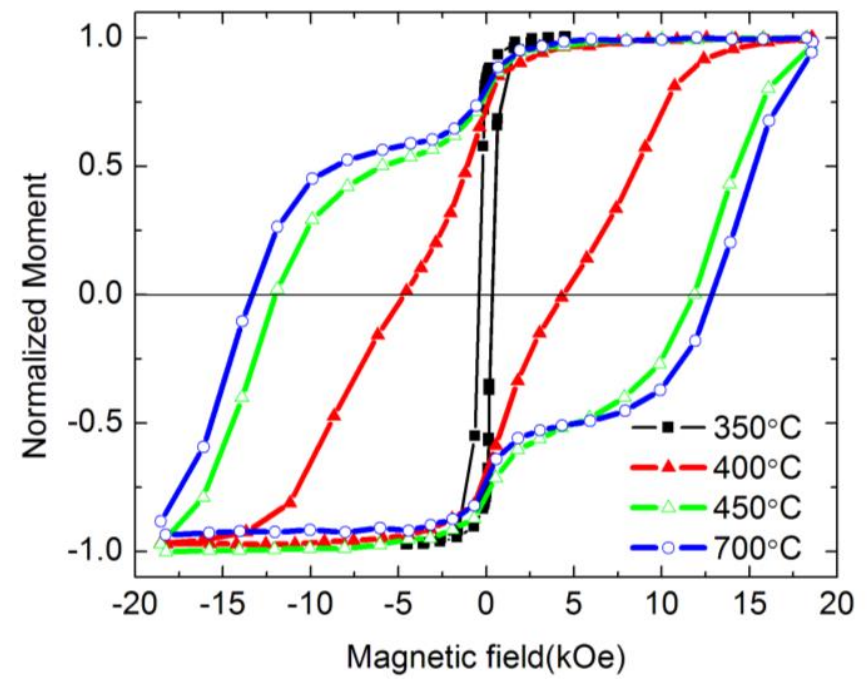

c)

Figure 4.20 In-plane magnetic loops for near equiatomic films with different thickness upon annealing at various temperatures: a) $23 \mathrm{~nm}$; b) $30 \mathrm{~nm}$; c) $93 \mathrm{~nm}$. 
Corresponding magnetic properties were monitored by VSM for these films. In fact, XRD is not sensitive to the presence of nanocrystalline phases due to the fact that it averages signals from $\sim \mathrm{mm}$ regions, and magnetic measurement can provide more sensitive information about the phases formed upon annealing. Fig.4.20 reports the in-plane hysteresis loops for films of different thicknesses subjected to various annealing temperatures. For all films, coercivity increased as increasing annealing temperatures; at $400^{\circ} \mathrm{C}$, coercivity of $2 \mathrm{kOe}$ or more was observed, even though XRD didn't detect the presence of the $\mathrm{L} 1_{0}$ phase for thinner films; for films of $23 \mathrm{~nm}$ and $30 \mathrm{~nm}$, after annealing to $600^{\circ} \mathrm{C}$, the butterfly feature in the loops suggests the appearance of magnetically decoupled phases. This can be the result of grain growth beyond the exchange length; thus, the magnetically soft phase can no longer be effectively coupled with the magnetically hard phase and switches independently of the latter $[32,33]$. A similar phenomenon was observed for all films but it appeared at lower temperatures for thicker films. Particularly, for the $93 \mathrm{~nm}$ film, a coercivity of $6.5 \mathrm{kOe}$ was already measured upon annealing at $400^{\circ} \mathrm{C}$, and it reached $\sim 12 \mathrm{kOe}$ for $450^{\circ} \mathrm{C}$, compared with about $10 \mathrm{kOe}$ at $\sim 600^{\circ} \mathrm{C}$ from acidic electrolytes $[5,6,9]$. Further annealing led to small increase of coercivity, indicating that the film was already fully magnetically saturated at the aging time we chose; this is consistent with the XRD patterns shown in Fig.4.18 b), at which fully developed $\mathrm{L} 1_{0}$ phase was indicated already at $450^{\circ} \mathrm{C}$.

Magnetization switching in Fe-Pt films is hindered by the pinning of magnetic domain walls at phase boundaries between the ordered and disordered phase, or between adjacent ordered grains with different orientation [1]. Coercivity is affected by the density of such defects, and it has been found to vary linearly with the volume fraction of ordered phase [34]. The variation of coercivity with annealing temperature (Fig.4.21) would therefore also represent the volume fraction of the ordered phase vs. annealing temperature.

The FCC to $\mathrm{L} 1_{0}$ transformation in Fe-Pt is a first order phase transition, occurring by 
nucleation and growth of the $\mathrm{L} 1_{0}$ phase in the parent phase [35]. This process would occur in parallel with grain growth of the FCC phase, but the former has a higher activation energy [36], and therefore occurs at a slower rate. This is confirmed by our observations that grain growth (Fig.4.19) started before any ordered phase was detected by an increase of coercivity (Fig.4.21). Thicker films were observed to order at lower annealing temperatures, probably due to a decrease in the energy barrier for nucleation of the $\mathrm{L} 1_{0}$ phase. Assuming that nucleation of the ordered phase occurs preferentially at defects, it follows that defects with higher energy are present in thicker films, originated perhaps by film stresses or voids [34].

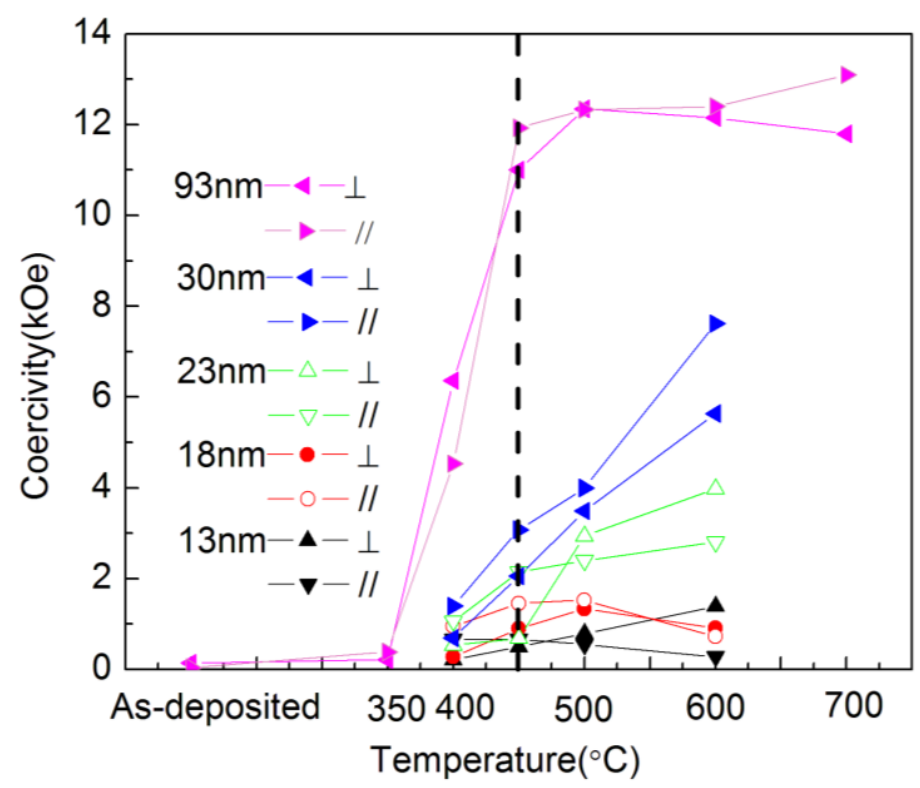

Figure 4.21 Coercivity of films with different thickness as a function of annealing temperatures.

\subsection{Bilayer and multilayer structures}

As discussed in section 4.2, the transition of random FCC to the ordered $\mathrm{L} 1_{0}$ phase, and thus magnetic hardening and increase in coercivity are highly dependent on the thickness of the films. Previous study has shown that the ordering kinetics for near equiatomic compositions is faster for slightly Fe rich films [37,38]. As a result, multilayer or sandwich structures have been made in order to study ordering kinetics of Fe-Pt alloys, usually using alternate pure $\mathrm{Fe}$ and pure $\mathrm{Pt}$, or pure $\mathrm{Fe}$ and $\mathrm{Fe}-\mathrm{Pt}$ alternate layers $[39,40]$. A decrease of the ordering temperature by such structures was 
confirmed.

In this section, we will study the compositional dependence of ordering by constructing bilayer and multilayer structures, using multilayers made of Fe rich (with about $75 \mathrm{at} . \%$ of $\mathrm{Fe})$ layers $\left(\mathrm{Fe}_{3} \mathrm{Pt}\right.$ in the following) with near equiatomic layers. We will first discuss the deposition of these structures by using a single electrodeposition process using the alkaline Fe-Pt solution discussed previously, and then the bilayer structures. An optimal $\mathrm{Fe}$ rich thickness will be determined and used for the multilayer structures for the study of effect of layer structure on the ordering process.

\subsubsection{Electrodeposition of compositionally graded multilayers}

The equiatomic FePt layer (a) was deposited at $-1.43 \mathrm{~V}$, and the $\mathrm{Fe}_{3} \mathrm{Pt}$ (b) layer at $-1.54 \mathrm{~V}$. The layer thickness of equiatomic FePt was fixed at $15 \mathrm{~nm}$ in all cases; in the bilayer structure, the Fe rich layer was 5, 10, 20 and 50nm, with Fe rich layer at the bottom and the equiatomic layer on the top. For multilayers, two configurations: a/b/a and $\mathrm{a} / \mathrm{b} / \mathrm{a} / \mathrm{b} / \mathrm{a}$ were constructed, and the thickness of $\mathrm{b}$ was $5 \mathrm{~nm}$ and $10 \mathrm{~nm}$, respectively (Fig.4.22). For the deposition of the bilayer and multilayer composite structures, a single step process was used by programming potential waveforms: the potentials applied and the deposition times were determined by the composition vs. potential and efficiency data shown previously. The switching time from one potential to another was less than 5 second, which was much shorter than deposition time; it is therefore reasonable to assume a sharp composition gradient within the interface. The possibility of partial $\mathrm{Fe}$ dissolution from the previous layer cannot be ruled out. However, as shown by EQCM in Fig.4.6, both applied potentials were more negative than the onset of Fe dissolution potential at $\sim-1.3 \mathrm{~V}$; for Fe-Pt, there is no appreciable dissolution even as positive as $-0.9 \mathrm{~V}$ (Fig.4.8). As a result, it is reasonable to assume that Fe dissolution can be neglected. 

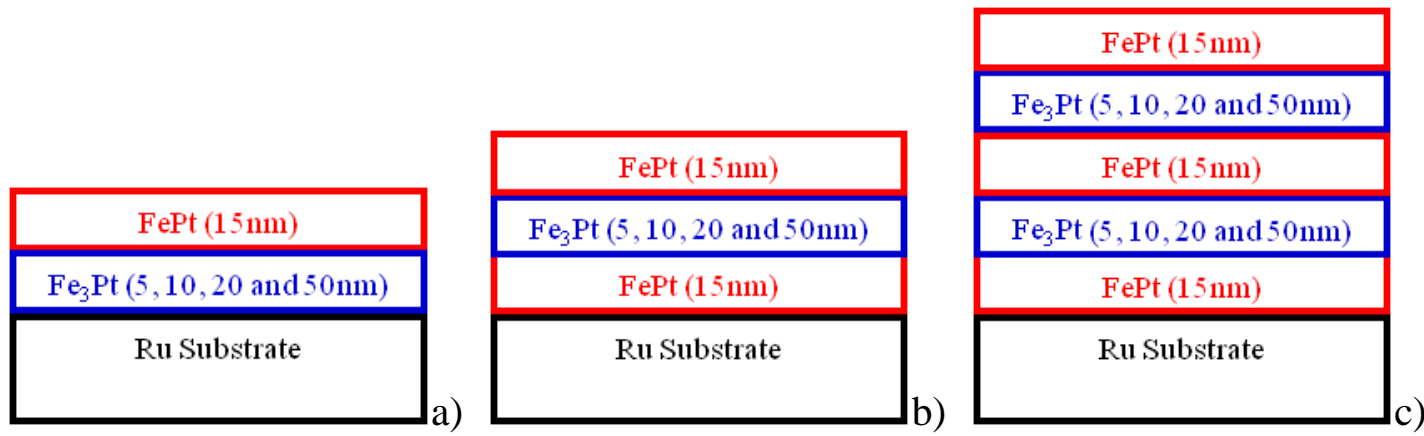

Figure 4.22 Schematic diagrams of bilayer, tri-layer and five-layer structures.

\subsubsection{Bilayer structures}

A current transient for a tri-layer structure is reported in Fig.4.23. The noisy signal was a result of the strong HER; most of the current measured is due to hydrogen generation.

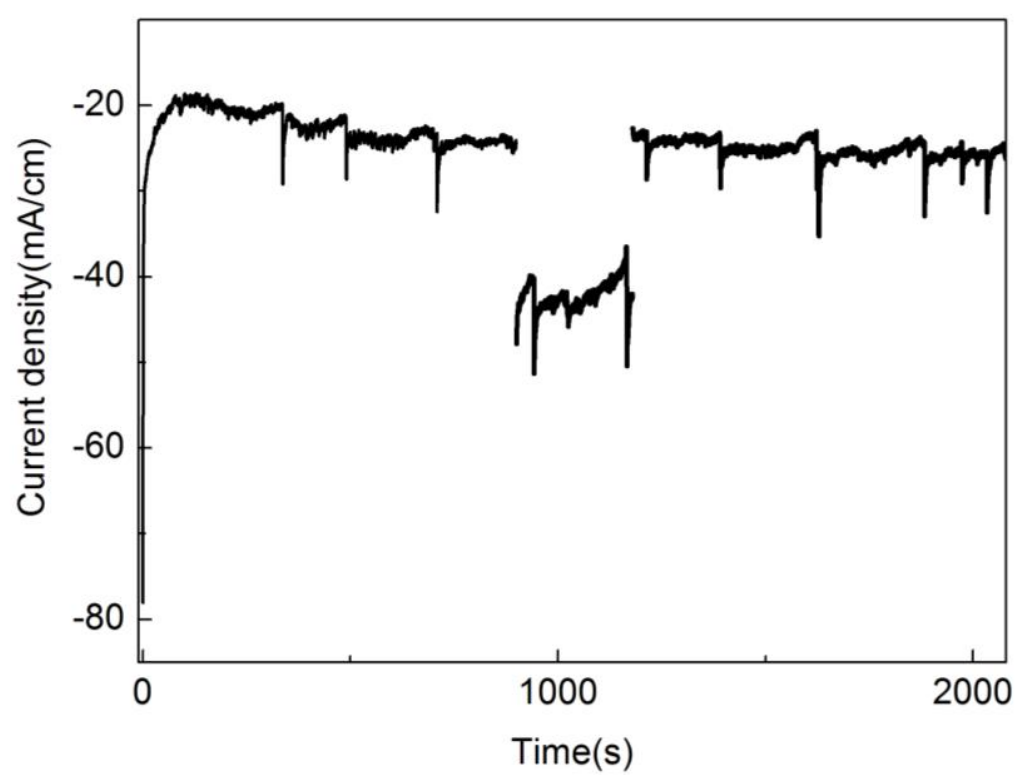

Figure 4.23 Current transient for a tri-layer structure $(\mathrm{Ru} / \mathrm{a} / \mathrm{b}(10 \mathrm{~nm}) / \mathrm{a})$.

The morphology of a FePt single layer $(30 \mathrm{~nm}), \mathrm{Fe}_{3} \mathrm{Pt}$ single layer $(65 \mathrm{~nm})$ and a bilayer of $\mathrm{Ru} / 20 \mathrm{~nm} \mathrm{Fe} 3 \mathrm{Pt}_{3} / 15 \mathrm{~nm} \mathrm{FePt}$ are shown in Fig.4.24. The apparent grain size for the $\mathrm{FePt}$ and $\mathrm{Fe}_{3} \mathrm{Pt}$ were estimated to be $\sim 22 \mathrm{~nm}$ and $45 \mathrm{~nm}$, respectively; the grain size for the top layer $\left(15 \mathrm{~nm} \mathrm{Fe}{ }_{3} \mathrm{Pt}\right)$ of the bilayer and multilayer structure was $\sim 23 \mathrm{~nm}$, similar to that of the single layer, suggesting that no appreciable roughening occurs due to the coupling of different layers. 

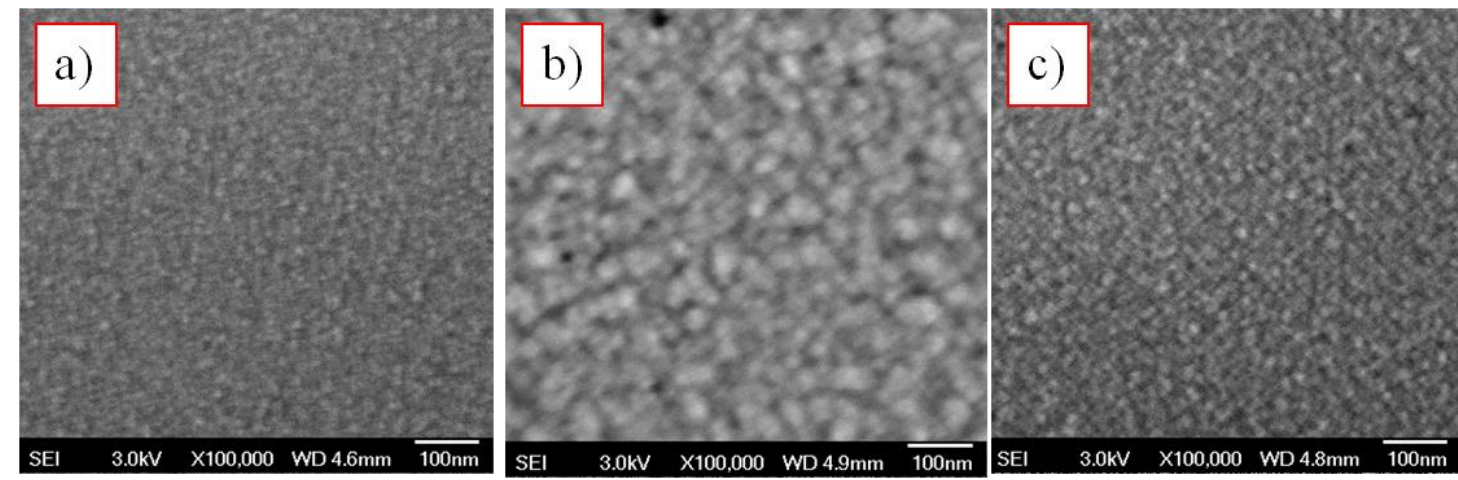

Figure 4.24 Morphology of a) $30 \mathrm{~nm} \mathrm{FePt} \mathrm{single} \mathrm{layer,} \mathrm{b)} 65 \mathrm{~nm} \mathrm{Fe}{ }_{3} \mathrm{Pt}$ single layer and c) a bilayer of $\mathrm{Ru} / 20 \mathrm{~nm} \mathrm{Fe} \mathrm{Pt}_{3} / 15 \mathrm{~nm} \mathrm{FePt}$ in as-deposited state.

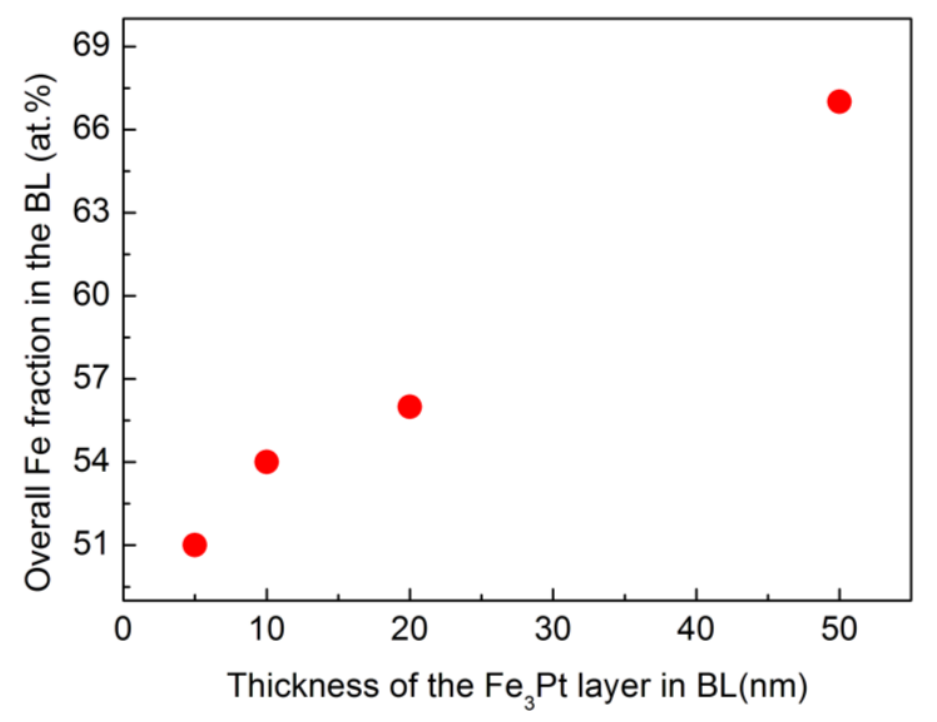

a)

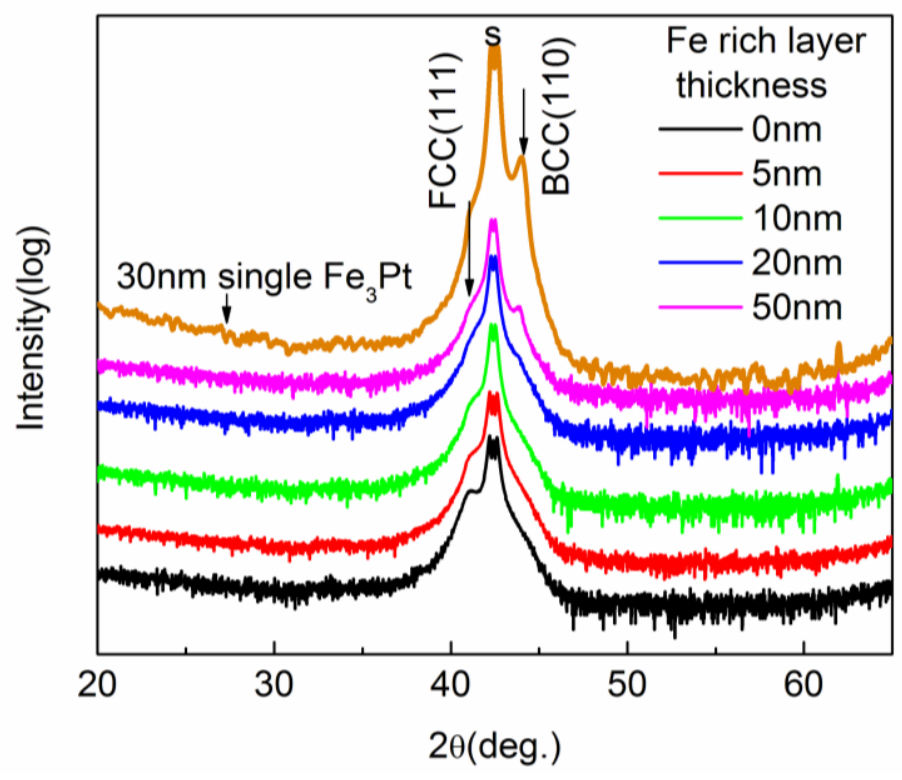

b)

Figure 4.25 a) Overall compositions for the bilayers; b) XRD patterns for a $15 \mathrm{~nm}$ single $\mathrm{FePt}$ layer, bilayers and a $30 \mathrm{~nm}$ single $\mathrm{Fe}_{3} \mathrm{Pt}$ layer. 
Fig.4.25 shows the overall composition for the bilayers, and XRD patterns for as-deposited films of a single layer $\mathrm{Fe}-\mathrm{Pt}$, single layer $\mathrm{Fe}_{3} \mathrm{Pt}$ and a series of bilayer. Similar to what we have observed for single layer films in Section 4.2, the FePt film showed a (111) orientation; as the thickness of Fe rich films increases, a shoulder around 44 degree was observed. It progressively increased in intensity and was clearly visible when Fe rich layer thickness increases to $20 \mathrm{~nm}$. This reflection is attributed to the (110) peak for the Fe rich layer; this is confirmed by the even more evident peak for the $65 \mathrm{~nm}$ single Fe rich layer, of which the peak was observed exactly the same angle. The attenuation of the (111) peak, however, cannot be explained with the thickness increase of Fe rich layer since the Fe-Pt layer is in fact on the top. We here tentatively assume that its crystallinity is weakened by depositing on the Fe rich layer.

To investigate the ordering kinetics, films were annealed in forming gas for $1 \mathrm{~h}$ at various temperatures. The corresponding XRD patterns are displayed in Fig.4.26. For all bilayers, an increase in the intensity of (111) peak was observed, similar to equiatomic single layers discussed in Section 4.2; this can be attributed to the grain growth upon annealing. For bilayers with $5 \mathrm{~nm}$ and $10 \mathrm{~nm}$ Fe rich layers, no other peaks were detected even after a $650^{\circ} \mathrm{C}$ annealing. However, for bilayers with $20 \mathrm{~nm}$ and $50 \mathrm{~nm}$ Fe rich layers, an additional peak appeared at $450^{\circ} \mathrm{C}$, and it was finally separated into two peaks after annealing at $650^{\circ} \mathrm{C}$. Meanwhile, the (110) peak attenuated as the appearance of the other peaks, and finally disappeared at $550^{\circ} \mathrm{C}$. According to the angle positions of the additional peaks, we assign the first appearing peak to be a textured FCC (200), which was then split into (200) and (002). 

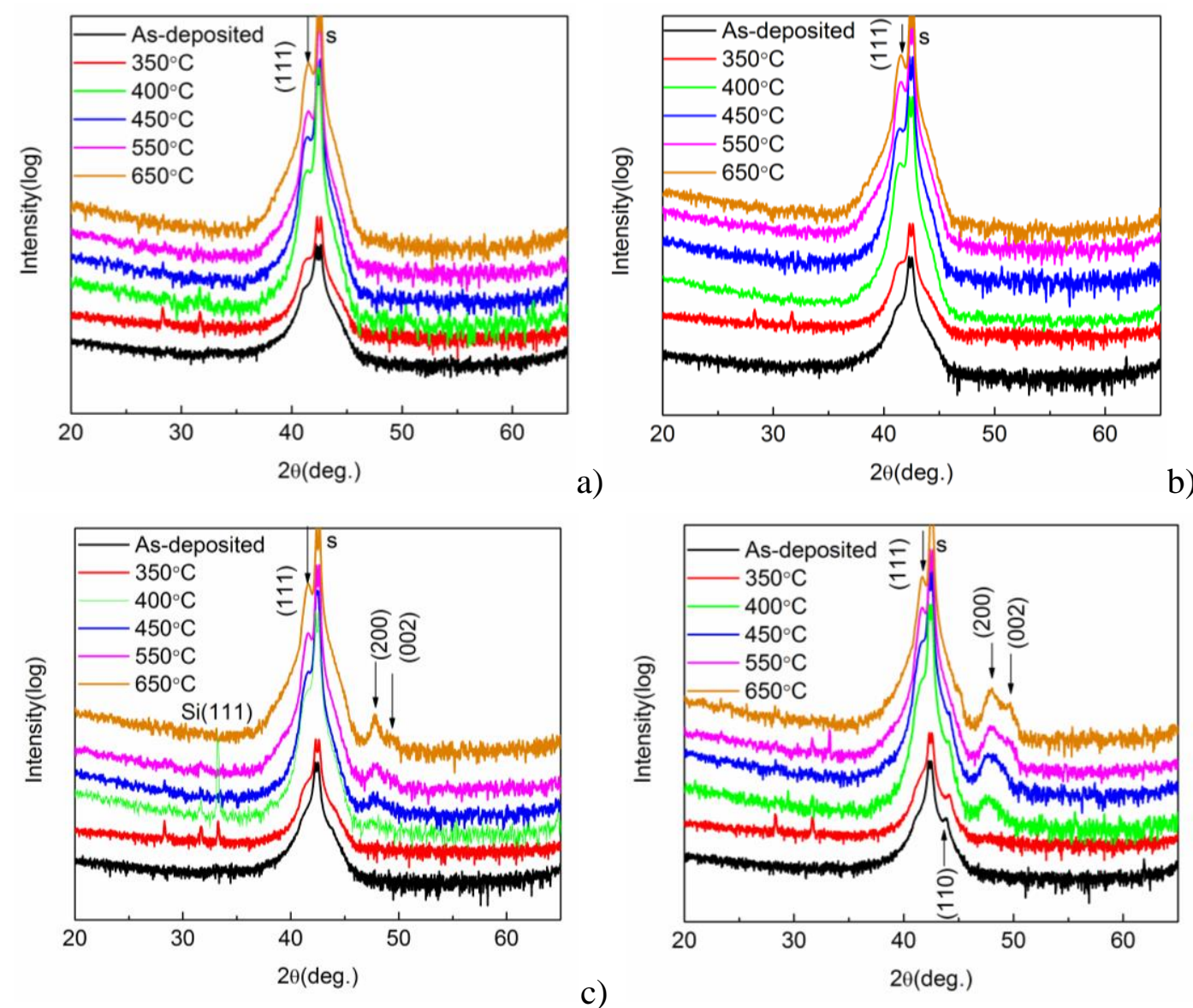

b)

Figure 4.26 XRD patterns for bilayers with different thickness upon annealing at various temperatures: a) $b=5 \mathrm{~nm}$; b) $b=10 \mathrm{~nm}$; c) $b=20 \mathrm{~nm}$; d) $b=50 \mathrm{~nm}$.

Assuming that this structure evolution is a phase transition into $\mathrm{L}_{0}$ structure, it is surprising that no other superlattice peaks, such as the usual (001) and (110), are observed as has been shown in section 4.2. To better understand this transition, a similar annealing experiment has been performed with a $65 \mathrm{~nm} \mathrm{Fe}{ }_{3} \mathrm{Pt}$ single layer. The corresponding XRD patterns are reported in Fig.4.27. The BCC (110) texture was clearly shown; additionally, there was a very weak shoulder at $\sim 42^{\circ}$ which is assigned to the FCC (111) in the as-deposited film. Upon annealing, a peak appeared at $400^{\circ} \mathrm{C}$, which finally separated into two well defined peaks at $650^{\circ} \mathrm{C}$, together with a peak at $24.2^{\circ}$. The BCC (110) peak was weakened following annealing and disappeared at $550^{\circ} \mathrm{C}$. Comparing with the bilayer XRD patterns for $20 \mathrm{~nm}$ and $50 \mathrm{~nm}$ thick $\mathrm{Fe}_{3} \mathrm{Pt}$ layers, they show a similar trend. We then assign those peaks as (001) at $24.2^{\circ}$, and a separation of (200) into (200) and (002), which, as a result, suggests a transition to $\mathrm{L} 1_{0}$ phase. This is highly unexpected since at $75 \mathrm{at} . \%$ of $\mathrm{Fe}$, the equilibrium phase 
diagram shows an ordered structure of $\mathrm{L1}_{2}$, which is a cubic structure, and the separation of (200) into (200) and (002) is not expected for a cubic phase. However, the phenomenon observed for the single Fe rich layer suggests that what has been observed for the bilayer structures may be due to the transition occurring in the $\mathrm{Fe}$ rich layer. The transition and the exact phase generated will be discussed in the following section on the microstructure of $\mathrm{Fe}_{3} \mathrm{Pt}$.

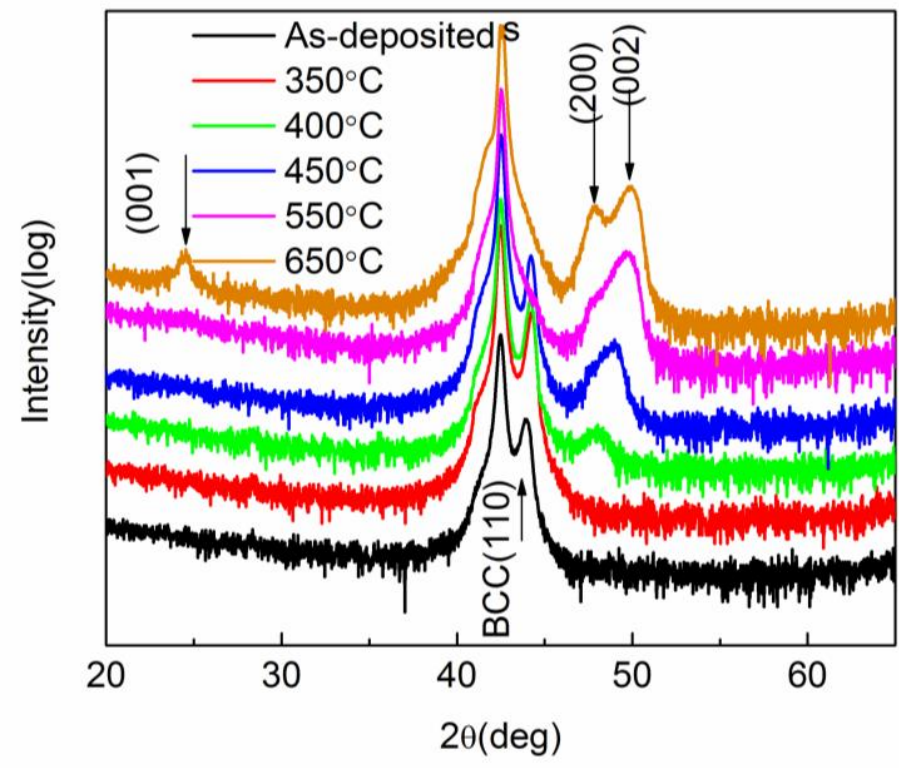

Figure 4.27 XRD patterns for a $65 \mathrm{~nm}$ single $\mathrm{Fe}_{3} \mathrm{Pt}$ film annealed at various temperatures.

The corresponding magnetic properties were monitored with VSM; the in-plane hysteresis loops for bilayers with $5 \mathrm{~nm}$ and $20 \mathrm{~nm}$ thick Fe rich layers are shown in Fig.4.28. For all the bilayers, coercivity slowly increased to $1 \mathrm{kOe}$ after $450^{\circ} \mathrm{C}$ annealing; however, significant increase of coercivty up to $\sim 7 \mathrm{kOe}$ was observed upon annealing at $550^{\circ} \mathrm{C}$, which was then further enhanced up to $12 \mathrm{kOe}$ at $650^{\circ} \mathrm{C}$. In Fig.4.29, a summary of the coercivity of the bilayers as a function of Fe rich layer thickness annealed at various temperatures is shown. It is clearly seen that above $450^{\circ} \mathrm{C}$, coercivity is greatly dependent on the thickness of the $\mathrm{Fe}$ rich layer: it increased up to $10 \mathrm{~nm}$ layer, and then decreased in coercivity, reaching approximately that of the FePt single layer. The coupling of $5 \mathrm{~nm}$ or $10 \mathrm{~nm}$ of Fe rich layer with a 15 $\mathrm{nm} \mathrm{FePt}$ layer is able to enhance the coercivity by $\sim 50 \%$ after 550 and $650^{\circ} \mathrm{C}$ annealing. 


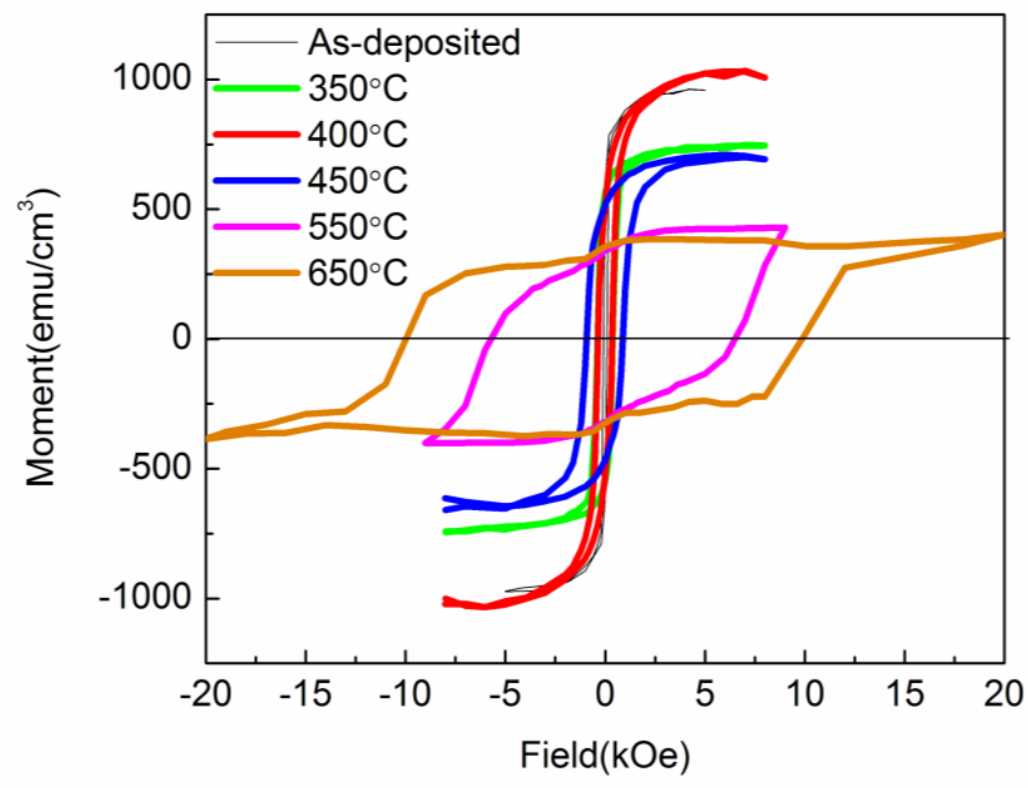

a)

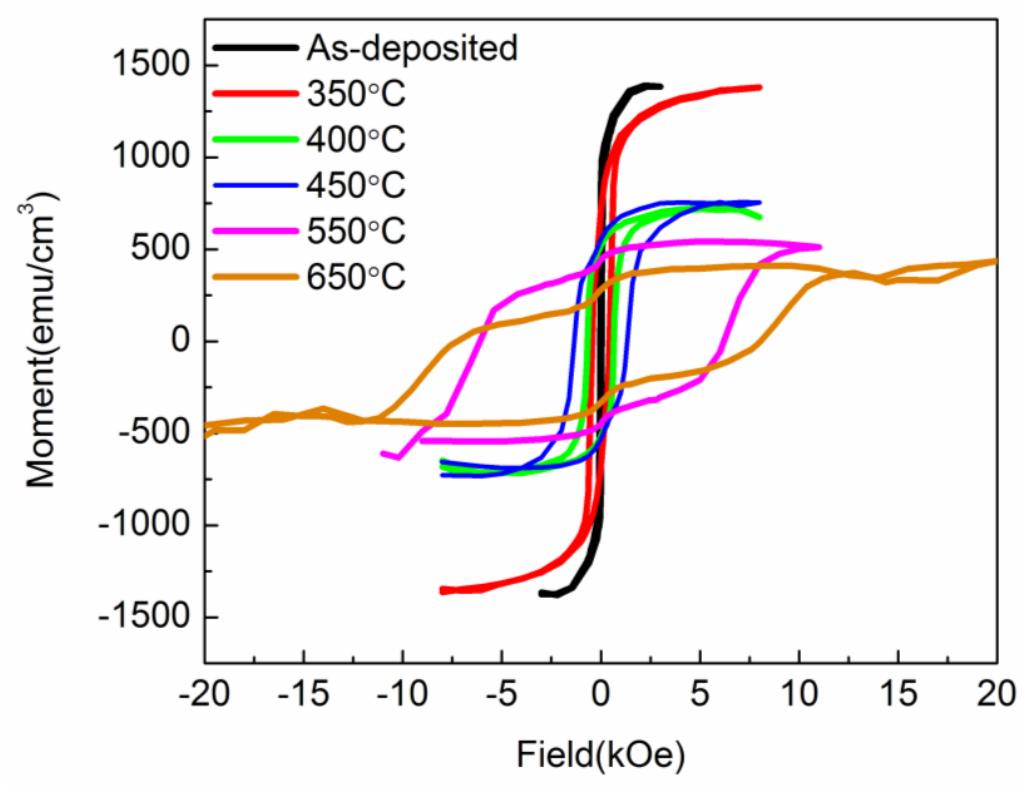

b)

Figure 4.28 In-plane hysteresis loops for bilayer structures annealed at various temperatures for $1 \mathrm{~h}$ with different Fe rich layer thicknesses: a) $b=5 \mathrm{~nm}$; $) b=20 \mathrm{~nm}$.

Previous studies on Fe-Pt films revealed that the kinetics of ordering is greatly dependent on the film thickness; thicker films are transformed to the $\mathrm{L}_{0}$ phase and develop high coercivities at lower temperatures [29,41] (see Section 4.2). The observed trend of Hc in bilayer films, however, cannot be simply attributed to film thickness effect. Thermal annealing activates diffusion phenomena, which result both in structural ordering and in interlayer diffusion. In bilayers with a Fe-rich layer of 5 or $10 \mathrm{~nm}$, the enhancement in magnetic hardening rate with respect to equiatomic 
single layers is probably related to induced ordering in the equiatomic layer caused by coupling with the Fe rich layer; diffusion phenomena in the latter are in fact activated at lower temperature and it is conceivable that the resulting change in lattice symmetry (FCC to FCT, confirmed in latter section of $\mathrm{Fe}_{3} \mathrm{Pt}$ ) would induce a similar transformation in the equiatomic layer. The small thickness of the Fe rich layer furthermore favors a strong coupling between the spins of the two layers, consistent with the hysteresis loops observed at 450 and $550^{\circ} \mathrm{C}$, showing a single phase magnetization switching behavior (Fig.4.28a). In parallel with phase transformation, interdiffusion between the two layers is also possible during annealing, which would result in a compositional gradient across the interface. The actual extent of the interdiffusion process is difficult to quantify since the defect structure of the two layers is unknown. Mixing of the equiatomic layer with a thin $(5-10 \mathrm{~nm})$ Fe-rich layer however may result in homogenization of the composition across the interface, resulting in a homogeneous alloy with a slight Fe excess, i.e. in the optimum compositional range for fast ordering kinetics and therefore capable to achieve higher coercivities at lower temperatures. As the thickness of the Fe rich layer increases beyond $10 \mathrm{~nm}$, interdiffusion may be insufficient to achieve complete homogenization, leaving therefore a fraction of the Fe rich layer unchanged. In this region the alloy composition may be too rich in Fe to allow transformation to the $\mathrm{L} 1_{0}$ phase, leading to a low coercivity. It is also possible that for Fe rich layers thicker than $20 \mathrm{~nm}$ the exchange coupling of the spins in the two layers is insufficient to warrant rigid spin rotation, leading to partially magnetically decoupled films, and in a lower overall coercivity. These findings are in agreement with the trends reported for $\mathrm{L}_{0} \mathrm{FePt} / \mathrm{Fe}$ bilayers $[38,39,42]$.

From the shape of the magnetic loops (Fig.4.28a)), bilayers with a $5 \mathrm{~nm}$ Fe rich layer showed single phase magnetization with only a faint shoulder in the second quadrant, suggesting good exchange coupling; as the Fe rich layer becomes thicker, two partially independent switching processes were observed, implying partial decoupling. The in-plane and perpendicular loops have similar behavior, indicative of an 
approximately isotropic magnetization response.

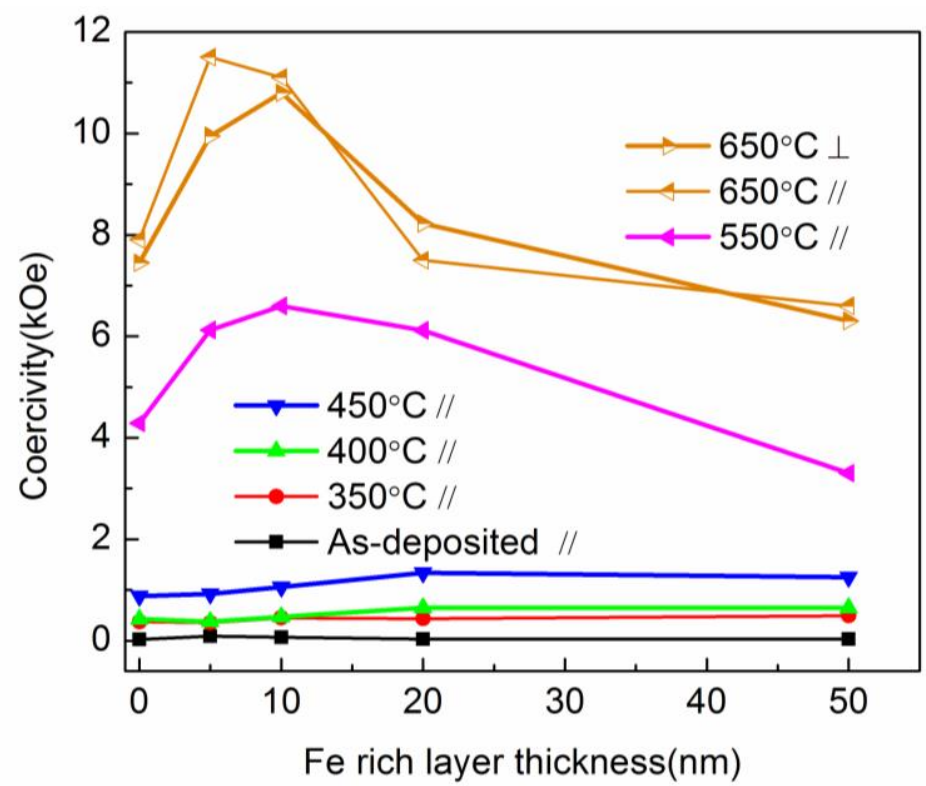

Figure 4.29 Coercivity for the bilayer structures with different Fe rich layer thicknesses annealed at elevated temperatures.

\subsubsection{Multilayer structures}

On discussing the bilayer structures above, it was found that by coupling an Fe rich layer with an appropriate thickness with a near equiatomic layer, the magnetic hardening can be enhanced with great increase in coercivity. In this section, we will study the effect of layer configuration on the ordering kinetics by constructing a tri-layer and five-layer. Their configurations have already been shown in Fig.4.22 b) and c). The FePt layer is fixed at $15 \mathrm{~nm}$, the same as in the bilayer structures; the Fe rich $\mathrm{Fe}_{3} \mathrm{Pt}$ layer is chosen as $5 \mathrm{~nm}$ and $10 \mathrm{~nm}$, according to the results for the bilayers.

The XRD patterns for the multilayers upon annealing at elevated temperatures are shown in Fig.4.30. Similar to the bilayers with the same thickness of Fe rich layers, upon annealing, the main feature observed is the intensity increase of the (111) alloy peak, which again can be attributed to the grain growth. The corresponding in-plane hysteresis loops are reported in Fig.4.31. Similar to the corresponding bilayers, a small increase in coercivity was seen up to $450{ }^{\circ} \mathrm{C}$; however, significant increase was observed at $550^{\circ} \mathrm{C}$ and $650^{\circ} \mathrm{C}$. For better comparison, the coercivity as a function of 
layer configuration is demonstrated in Fig.4.32. It is clearly shown that coercivity is roughly independent of the layer configurations (the reason for the abnormous reduction of the tri-layer after annealing at $650{ }^{\circ} \mathrm{C}$ is not clear). In other words, bilayers by coupling an appropriate thickness of $\mathrm{Fe}$ rich is sufficient to greatly enhance the coercivity, while at the same time keeping the total thickness of the whole structure within several tens of nm.
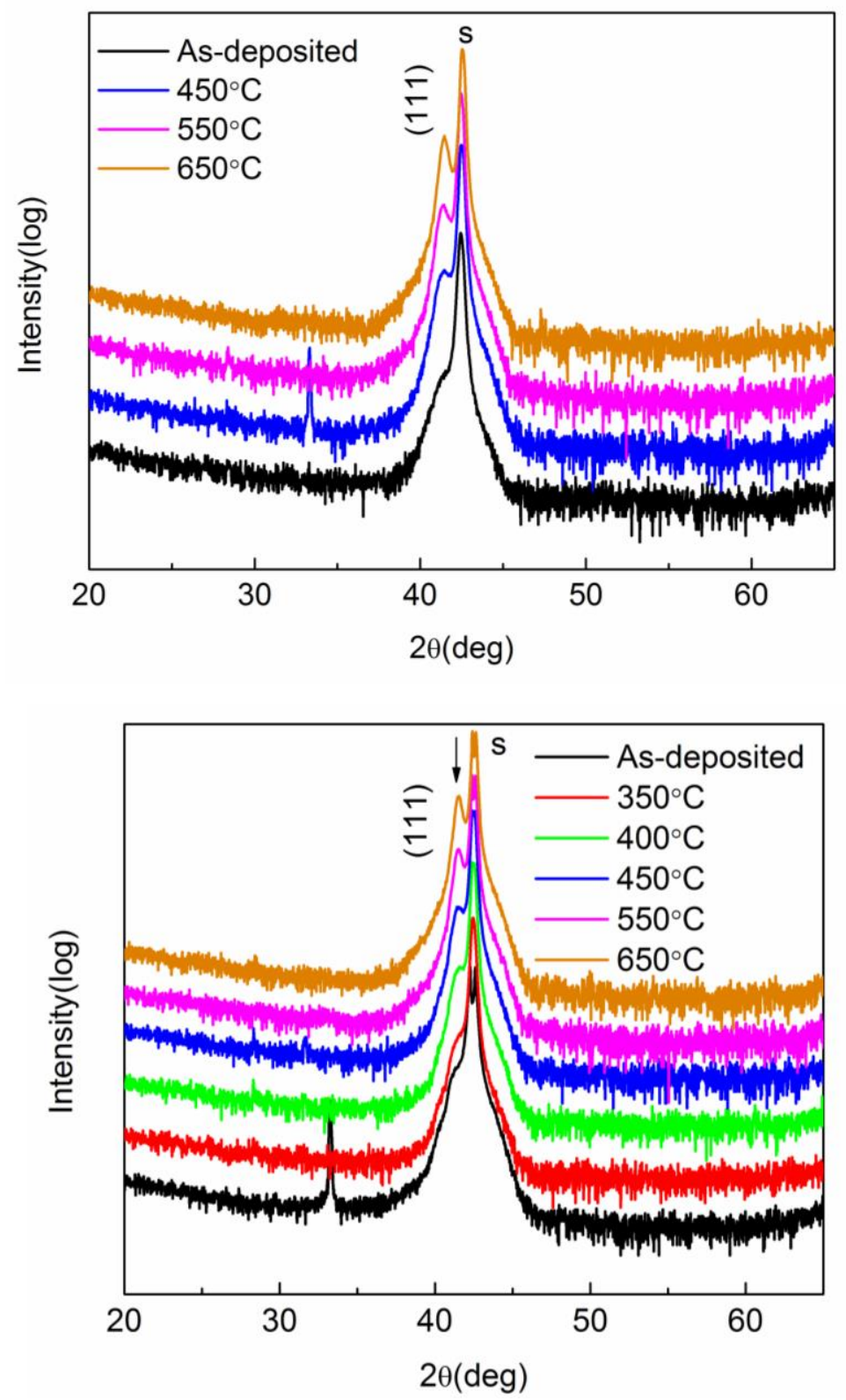

a)

b)

Figure 4.30 XRD patterns for the five-layer structures with different thicknesses of Fe rich layers annealed at elevated temperatures: a) $\mathrm{Ru} / \mathrm{a} / \mathrm{b} / \mathrm{a} / \mathrm{b} / \mathrm{a}(\mathrm{b}=5 \mathrm{~nm}) ; \mathrm{b}) \mathrm{Ru} / \mathrm{a} / \mathrm{b} / \mathrm{a} / \mathrm{b} / \mathrm{a}(\mathrm{b}=10 \mathrm{~nm})$ 


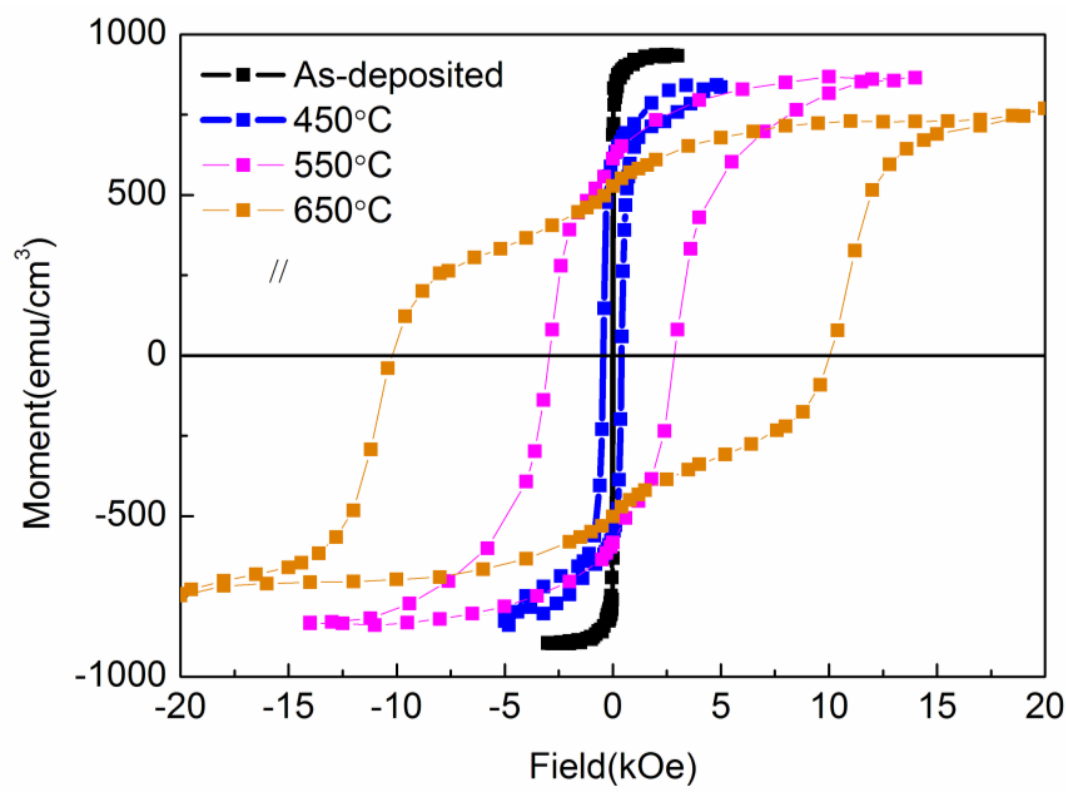

a)

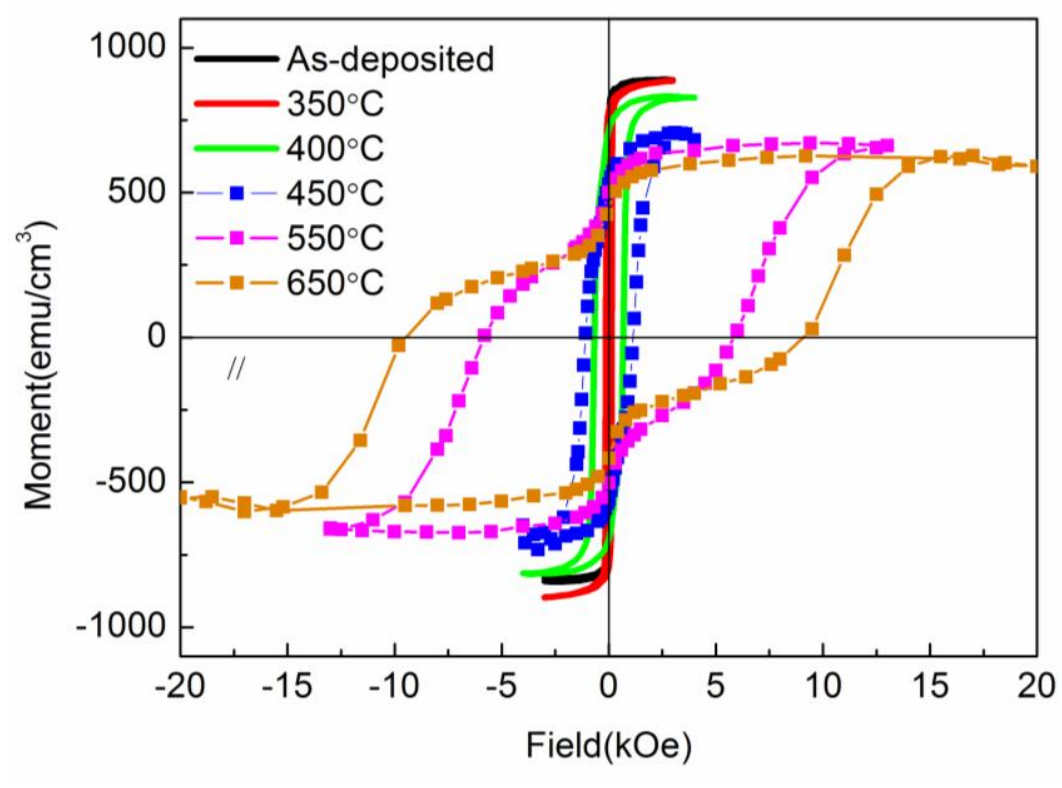

b)

Figure 4.31 In-plane hysteresis loops for the five-layer structures with different thicknesses of Fe rich layers annealed at elevated temperatures: a) $\mathrm{Ru} / \mathrm{a} / \mathrm{b} / \mathrm{a} / \mathrm{b} / \mathrm{a}(\mathrm{b}=5 \mathrm{~nm}) ; \mathrm{b}) \mathrm{Ru} / \mathrm{a} / \mathrm{b} / \mathrm{a} / \mathrm{b} / \mathrm{a}(\mathrm{b}=10 \mathrm{~nm})$ 


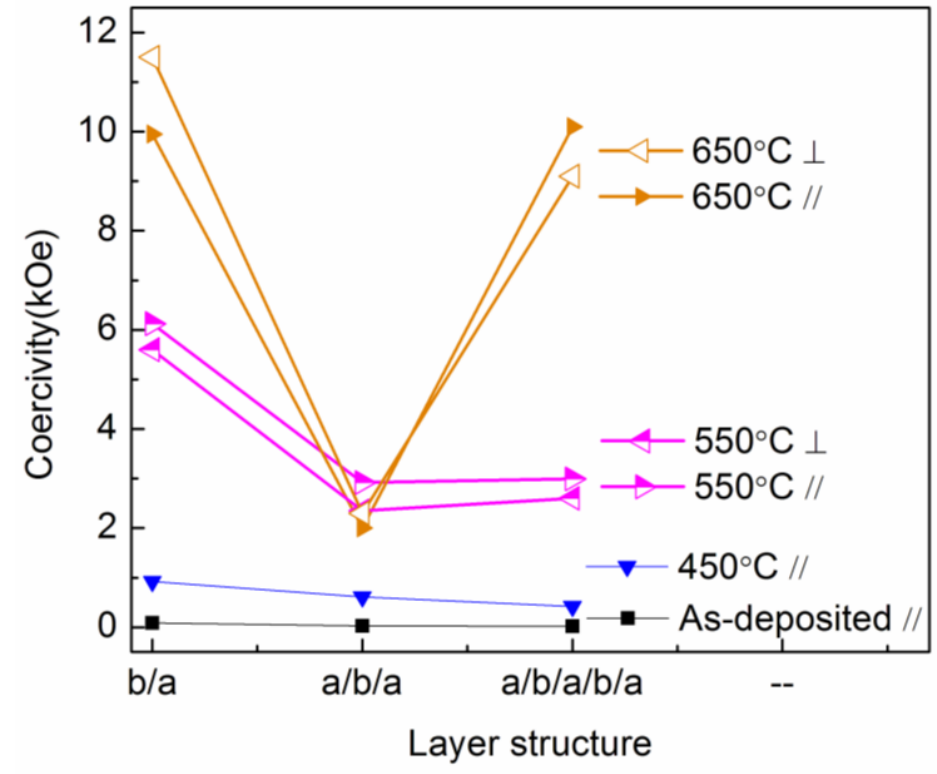

a)

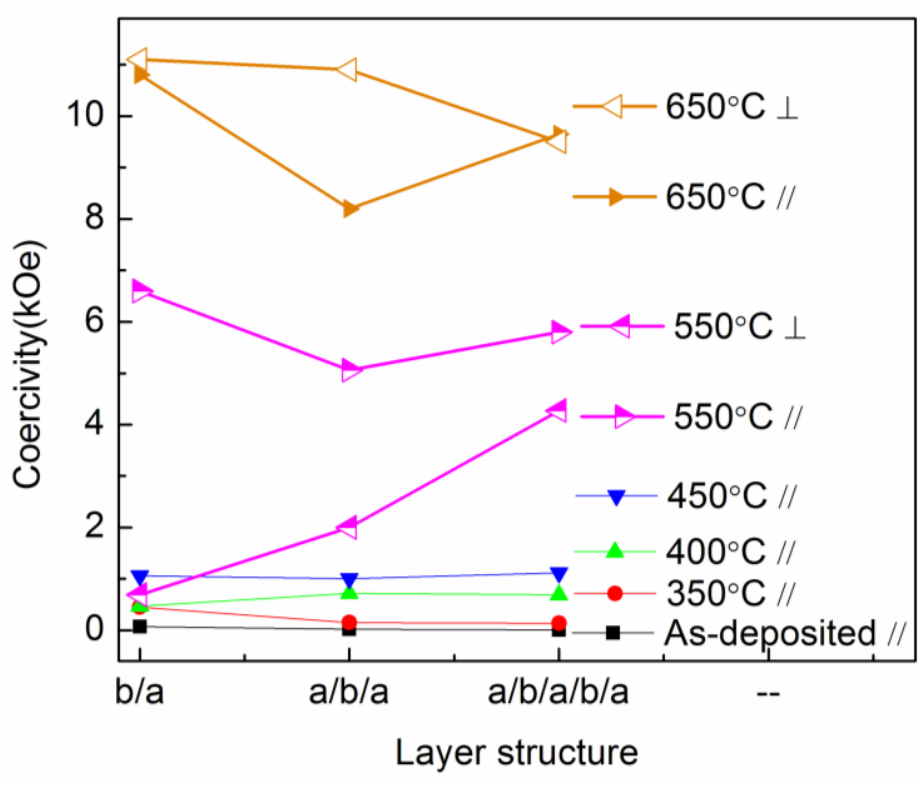

b)

Figure 4.32 coercivity as a function of layer configurations annealed at various temperatures: a) $\mathrm{b}=5 \mathrm{~nm} ; \mathrm{b}) \mathrm{b}=10 \mathrm{~nm}$.

The hysteresis loops for the multilayers show a behavior similar to the bilayers with Fe rich layer of $5 \mathrm{~nm}$ and $10 \mathrm{~nm}$, with the $5 \mathrm{~nm}$ one showing more or less a single switching process and the $10 \mathrm{~nm}$ one a clear two-stage switching process. In order to study interactions in a ferromagnetic material, the $\delta M$ method is often used, which compares the magnetization and demagnetization curves [43,44]. $\delta M$ is defined as

$$
\delta M=M_{d}-\left(1-2 M_{r}\right)
$$


where $M_{d}$ is the reduced dc demagnetization remanence curve obtained starting from a negative saturation field, and $\mathrm{M}_{\mathrm{r}}$ the reduced isothermal remanence curve starting from a demagnetized state.

Shown in Fig.4.33 are the $\delta M$ curves for the two five layer structures with $5 \mathrm{~nm}$ and $10 \mathrm{~nm}$ Fe rich layer thickness, respectively. A positive $\delta M$ was observed for both of the films, suggesting the predominance of exchange coupling intergrain interactions; the larger magnitude of the positive peak in the $\delta M$ curve for the $5 \mathrm{~nm} \mathrm{Fe}$ rich layer film implies stronger interaction than the $10 \mathrm{~nm}$ film, consistent with the observed hysteresis loops. In order to determine the origin of these interactions it is necessary to closely examine the bi- and multilayer microstructure. The exchange coupling could in fact originate within the single layer, through transformed and untransformed (or partially transformed) regions, or between the various layers. The maximum thickness for effective exchange coupling between the hard $\mathrm{L}_{0}$ and the FCC soft phase as given by Kneller and Hawig is $8 \mathrm{~nm}$ [32]. Under the assumption that the equiatomic layer is fully ordered, it is expected that the $5 \mathrm{~nm}$ thick Fe-rich layer should be strongly exchange coupled, while the $10 \mathrm{~nm}$ layer should be partly decoupled. This ideal behavior is not observed, since the $5 \mathrm{~nm}$ layer is not perfectly coupled, and exchange coupling is still observed for a $10 \mathrm{~nm}$ thick layer. This is probably due to the fact that in our films phase transformations and interdiffusion occur in parallel during thermal annealing, and the $\delta M$ method cannot separate the two effects. Previous work [33] has shown that exchange coupling could be extended beyond the Kneller and Hawig limit if a graded interface is obtained via interlayer diffusion. Our films may exhibit this type of behavior. 


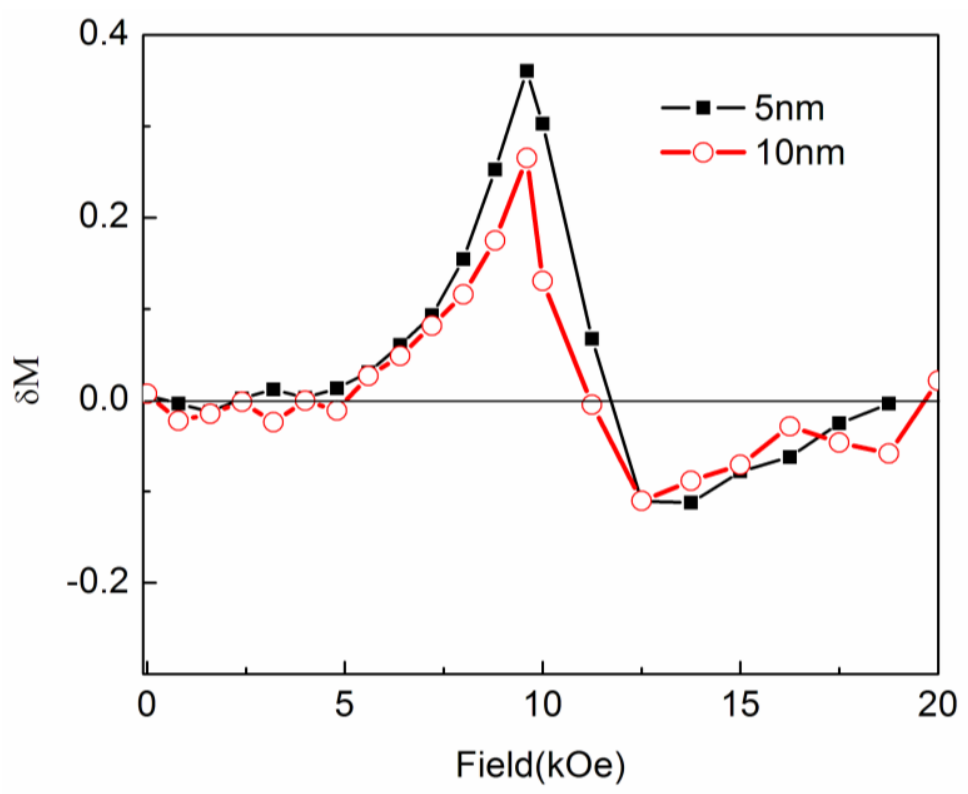

Figure $4.33 \delta \mathrm{M}$ curves for the two five-layer structures after annealing at $650^{\circ} \mathrm{C}$

\subsection{Microstructure of FePt}

In this section, the microstructure of near equiatomic FePt is discussed. A FePt films of $\sim 55 \mathrm{~nm}$ was characterized as-deposited and after annealing at $700^{\circ} \mathrm{C}$ following the annealing procedure in the experimental section. The magnetic loops are shown in Fig.4.34, which confirm achievement of high coercivity as expected.

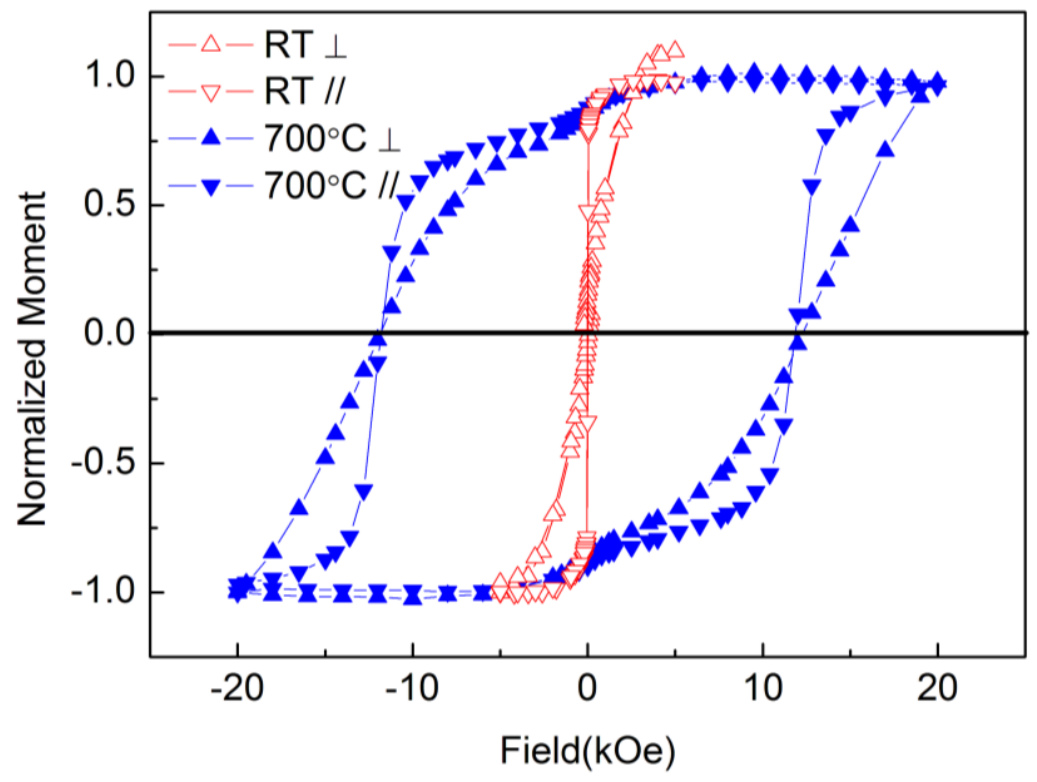

Figure 4.34 Hysteresis loops for $\sim 55 \mathrm{~nm}$ FePt film as-deposited and after annealing at $700^{\circ} \mathrm{C}$. 
The microstructure and corresponding SAED for the as-deposited and $700^{\circ} \mathrm{C}$ annealing alloy are shown in Fig.4.35. As-deposited film showed uniform grain sizes, averaging $~ 5 \mathrm{~nm}$; SAED confirms the presence of random FCC, with only fundamental peaks. After annealing, superlattice peaks were observed, indicating the transformation of $\mathrm{A} 1$ to $\mathrm{L1}_{0}$. Together with the transition, grain growth also occurs, resulting in a grain size of $\sim 25 \mathrm{~nm}$.

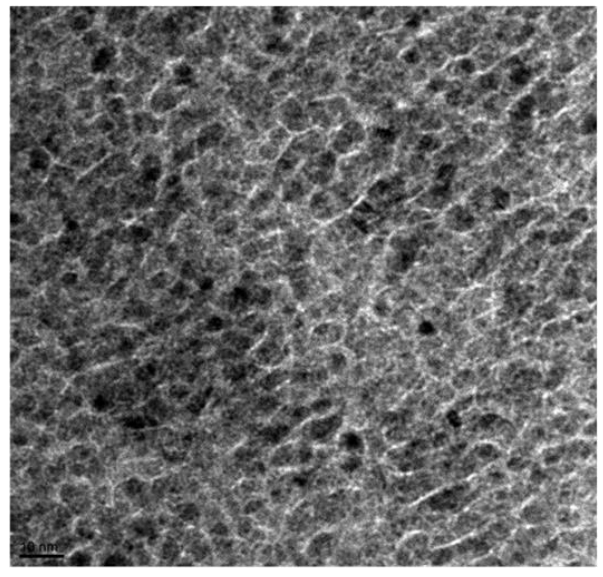

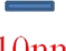

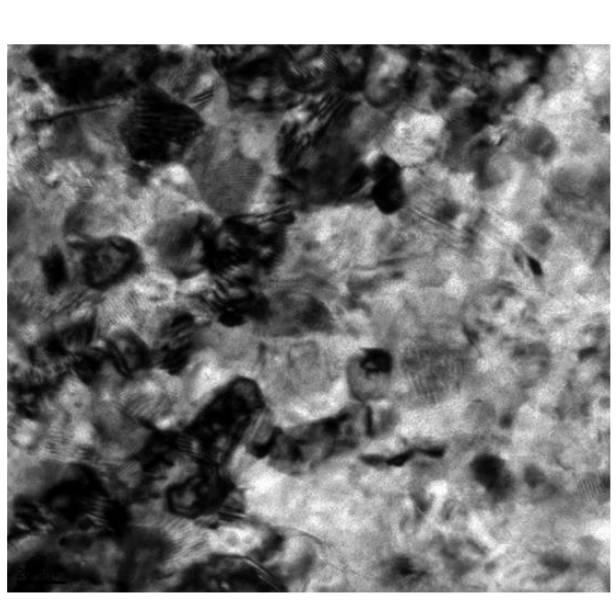

$20 \mathrm{~nm}$

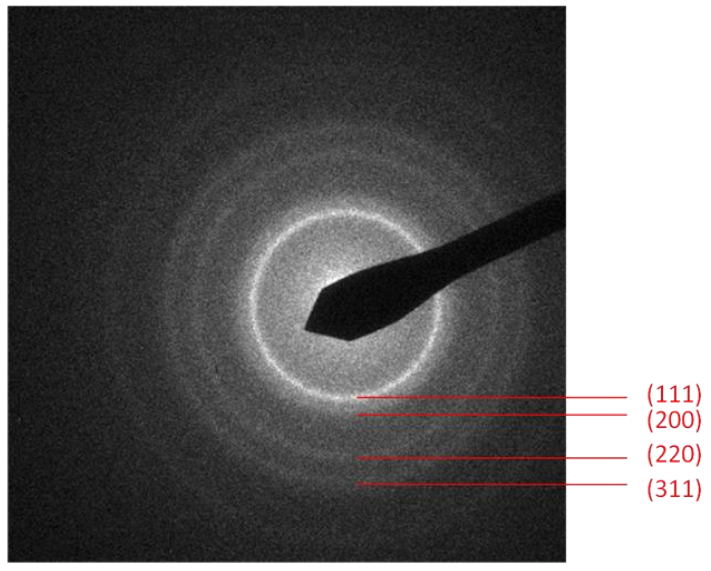

a)

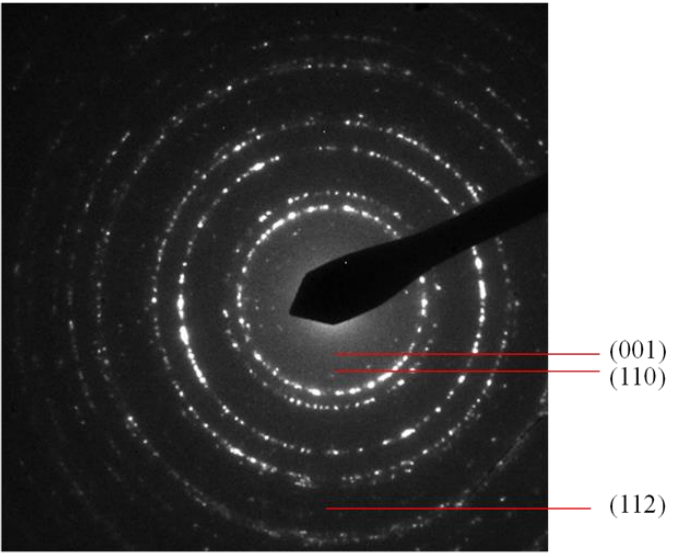

b)

Figure 4.35 TEM image and the corresponding SAED for: a) as-deposited; b) after $700^{\circ} \mathrm{C}$ annealing.

The dark field technique described in Chapter 2 was used to highlight the regions showing (001) reflection. As can be seen in Fig.4.36, the (001) grains were bright; highlighted grain sizes varied from several $\mathrm{nm}$ to tens of $\mathrm{nm}$, indicating a nucleation and grain growth transformation process, consistent with the $\mathrm{A} 1$ to $\mathrm{L} 1_{0}$ transition. 
Notice that the highlighted area was obtained by using only a fraction of the (001) ring, thus it is reasonable to assume that the transformed area fraction is larger than it was shown.

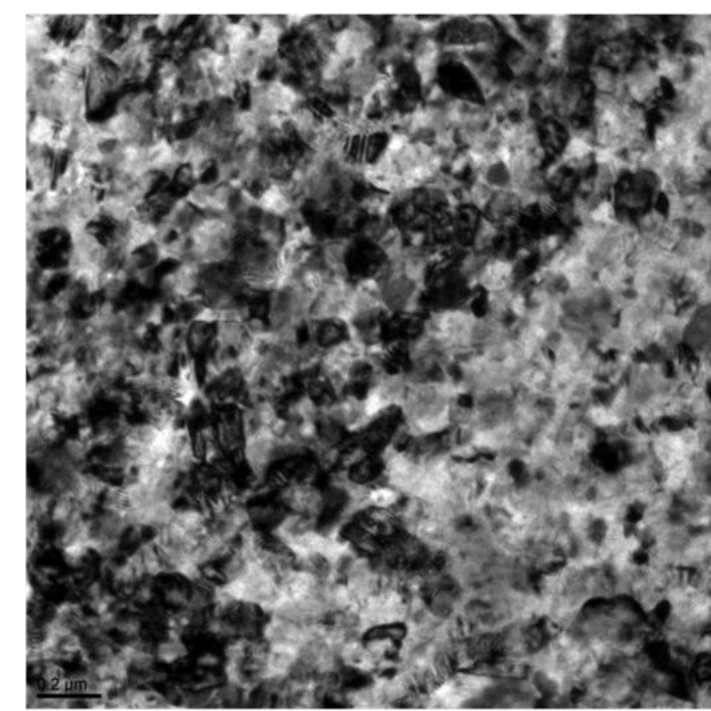

$200 \mathrm{~nm}$
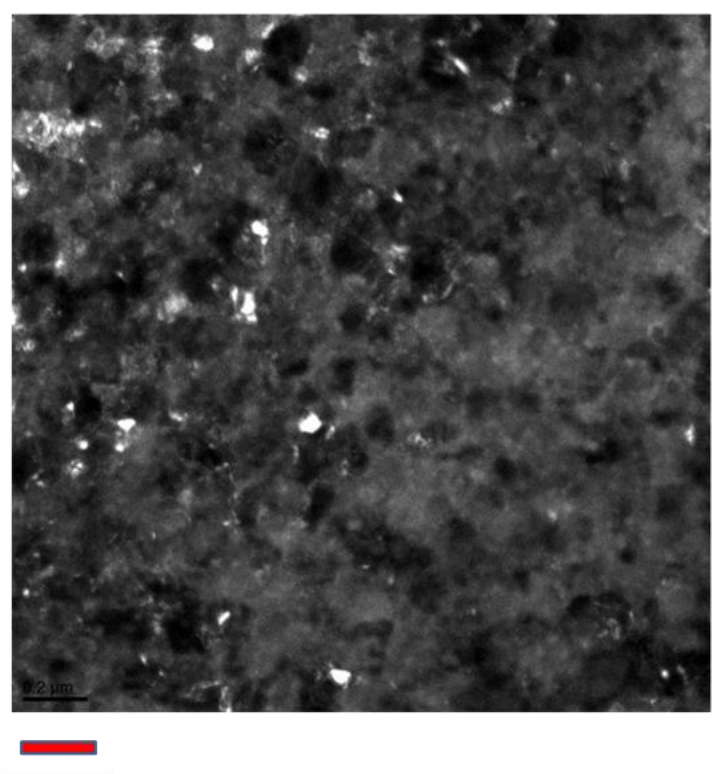

$200 \mathrm{~nm}$

Figure 4.36 BF image and corresponding DF image with a fraction of the (001) diffraction ring for the film after $700^{\circ} \mathrm{C}$ annealing.

\subsection{Crystal structure, microstructure of $\mathrm{Fe}$ rich and $\mathrm{Fe}_{3} \mathrm{Pt}$ alloys}

\subsubsection{Fe rich alloys with various Fe fractions}

Fe rich films with various $\mathrm{Fe}$ atomic fractions were electrodeposited under constant potentials. Fig.4.37 shows the XRD patterns for the as-deposited films with corresponding compositions. 


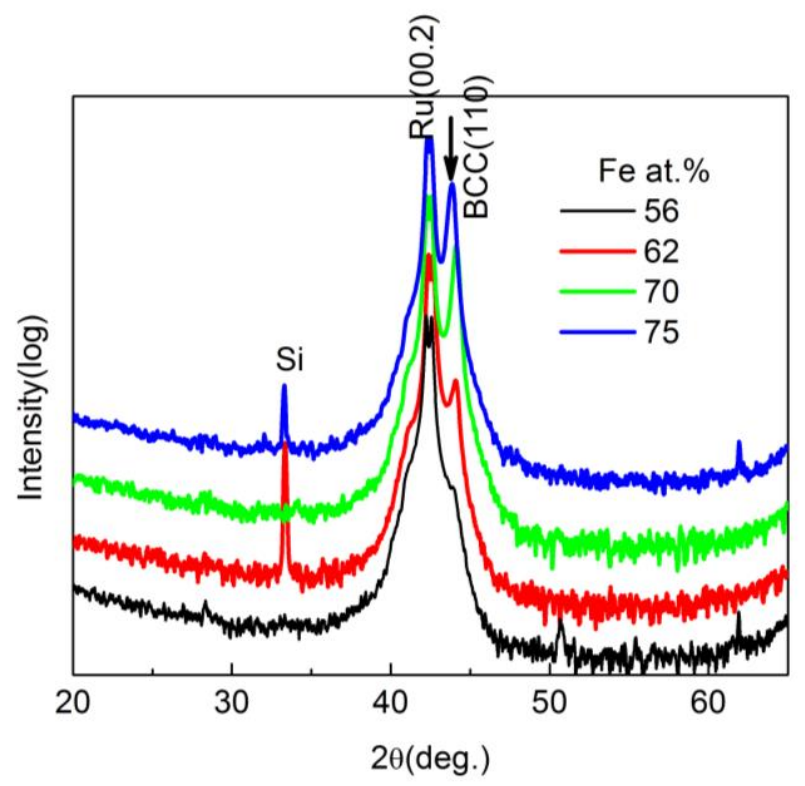

Figure 4.37 XRD patterns for as-deposited films with various Fe fraction (films $\sim 45-60 \mathrm{~nm}$ ).

In all these films, the FCC (111) peak around 41.5 degrees was not shown, or overlaps with the substrate peak; for the $56 \%$ one, a very weak shoulder for BCC (110) appeared; as Fe fraction increases, the shoulder evolved into an evident peak. There is a little shift in this peak, which may be due to the decrease in lattice parameter with increasing Fe, or to the superposition of the BCC peak, which becomes stronger, with the relatively unchanged substrate peak $\left(\sim 42.4^{\circ}\right)$.

The XRD patterns for films with various Fe fractions after annealing at elevated temperatures are shown in Fig.4.38. Upon annealing, several features were observed: a) a peak, which is identified as FCC (200), appeared after annealing at $400^{\circ} \mathrm{C}$, and it shifted to higher angle with increasing Fe fraction; b) at about $550^{\circ} \mathrm{C}$, the (200) peak split into two peaks, which corresponds to the separation of (200) into (200) and (002); the (002) peak became more evident after $650^{\circ} \mathrm{C}$ annealing; c) for 56 at. \% , it is almost pure (200) with a very weak shoulder of (002); with increasing Fe fraction, the ratio of (002) to (200) became larger, which may imply a stronger out of plane texture than in-plane for increasing Fe content; d) for films with $56 \%$ and $62 \%$ of $\mathrm{Fe}$, the FCC (111) shoulder was observed after annealing at $450^{\circ} \mathrm{C}$; it became more apparent at higher annealing temperatures; with increasing Fe fraction, the (111) peak 

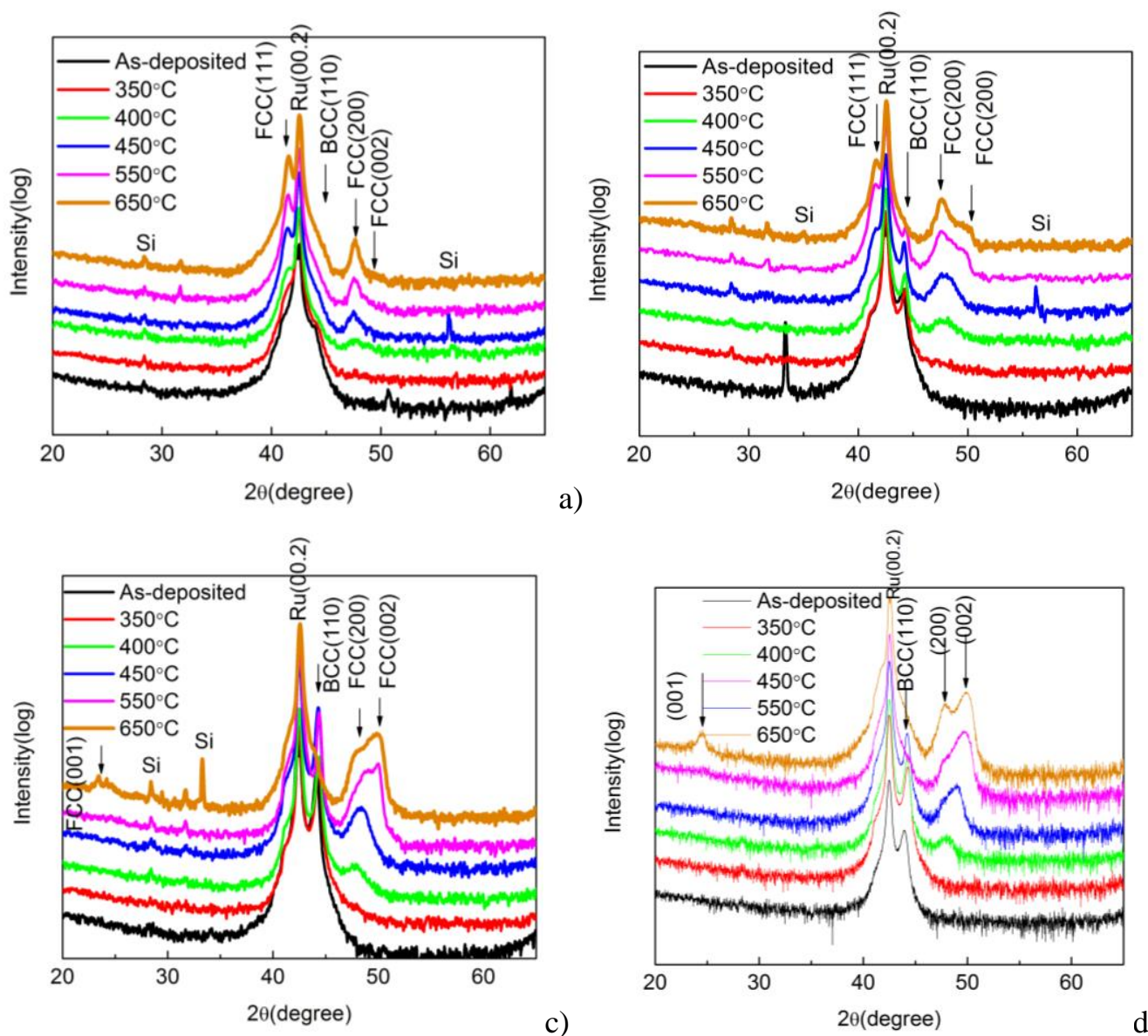

b)

Figure 4.38 XRD patterns for films $(45-65 \mathrm{~nm})$ with various $\mathrm{Fe}$ fractions after annealing at different temperatures with various Fe contents: a) 56 at.\%; b) 62 at.\%; c) 70 at.\%; d) 75 at.\%.

became less intense while the (200) (before separation into (200) and (002)) or (200) and (002) (after separation) became more intense (Fig.4.39); e) for films showing the BCC (110) peak in the as-deposited state, the BCC (110) peak became weaker with increasing annealing temperatures, and it disappeared after $650^{\circ} \mathrm{C}$. 


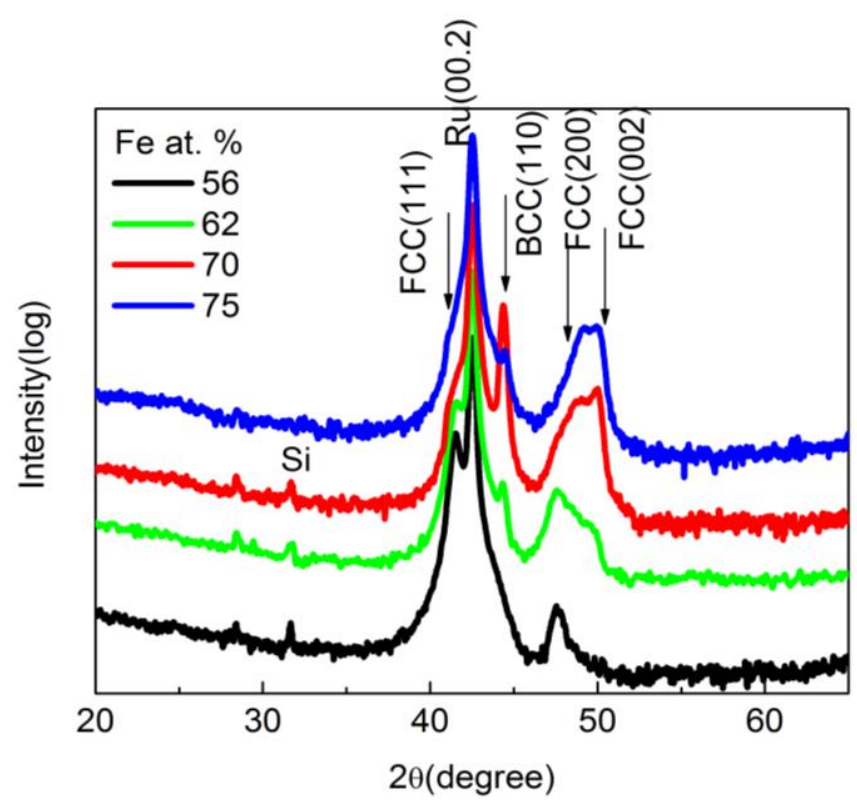

a)

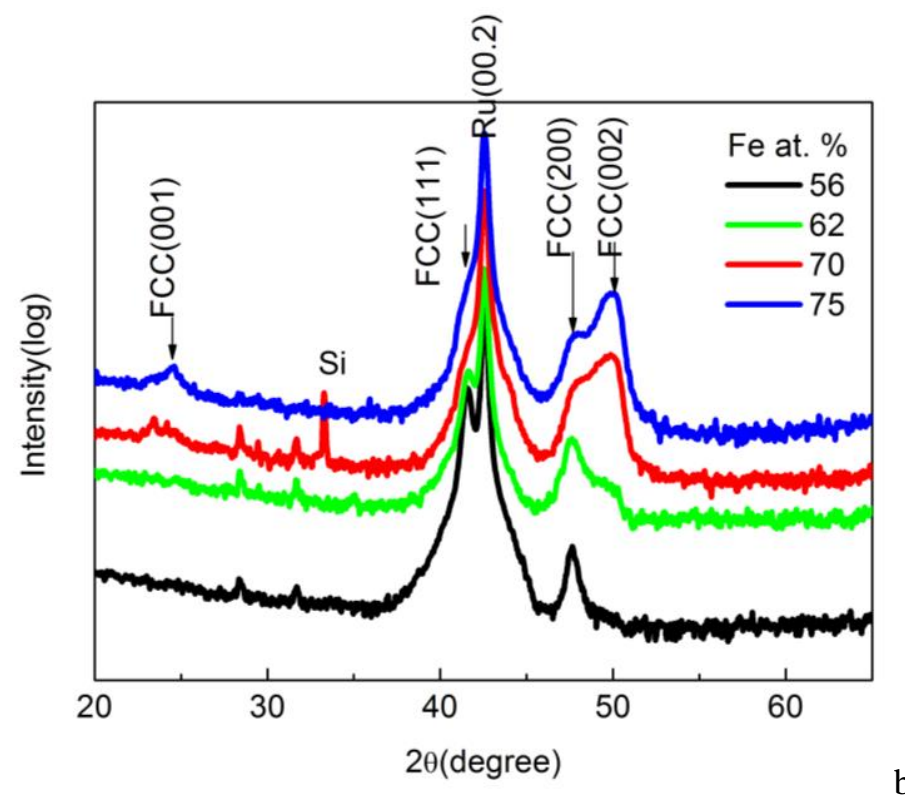

b)

Figure 4.39 Comparison of XRD patterns for films with various Fe fractions after annealing at: a) $550^{\circ} \mathrm{C}$; b) $650^{\circ} \mathrm{C}$ (extracted from Fig.4.38).

Fig.4.40 shows the in-plane and out of plane magnetic hysteresis loops for films with different $\mathrm{Fe}$ fractions. In as-deposited films, the saturation magnetization $\mathrm{M}_{\mathrm{s}}$ increased with increasing Fe fraction, which is consistent with literature [37]. In parallel, the coercivity after annealing at the same temperature decreased from the $56 \mathrm{Fe} \%$ to the $70 \%$; there is a little increase for the $75 \mathrm{Fe} \%$ compared with the $70 \%$ one. For films with $56 \%$ and $62 \%$ of $\mathrm{Fe}$, the in-plane coercivity is about two times larger than the out of plane one at the same temperature. This differs from the isotropic behavior of 

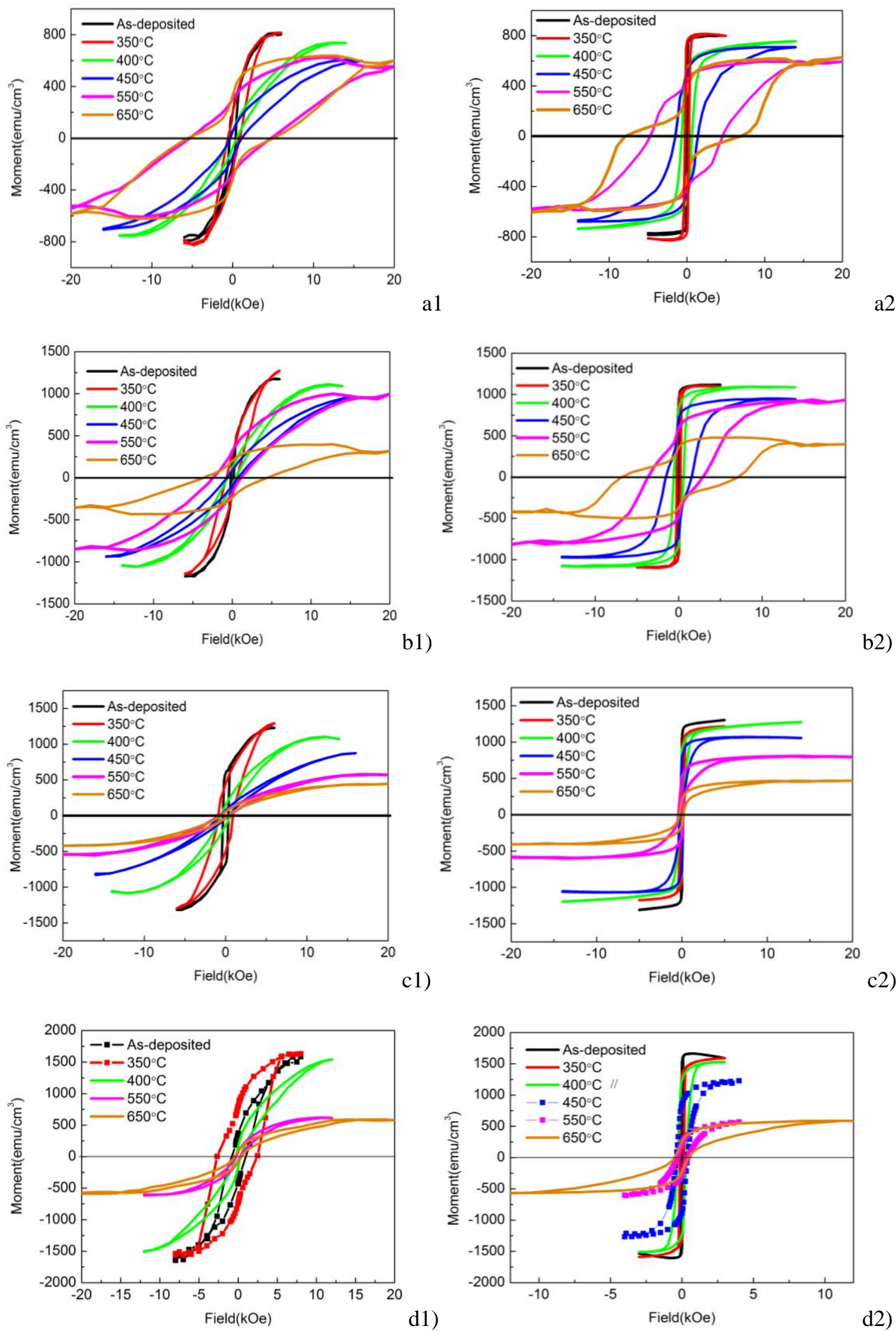

d2)

Figure 4.40 Out of plane and in-plane hysteresis loops for films with various $\mathrm{Fe}$ fractions: a1) $56 \% \perp$; a2) $56 \% / /$; b1) $62 \% \perp$; b2) $62 \% / /$; c1) $70 \% \perp$; c2) $70 \% / /$; d1) $75 \% \perp$; d2) $75 \% / /$.

equiatomic Fe-Pt films deposited on this Ru substrate, which usually induces the (111) 
orientation as shown previously in Section 4.2, and leads to similar coercivity in both directions. However, the larger in-plane than out of plane coercivity is consistent with what was observed in the XRD patterns shown in Fig.4.38 and Fig.4.39. For the 56\% Fe film, after the (200) peak appeared upon annealing at $400^{\circ} \mathrm{C}$, only the (200) was very evident, while only a faint (002) was observed; for the $62 \%$ Fe rich film, the (002) peak was well defined, and the ratio of (002) to (200) became larger with increasing temperature, but the (200) was still the dominant orientation. For the $70 \%$ Fe film, the (002) peak was stronger than the (200); however, the overall $\mathrm{L}_{0}$ phase volume fraction may be small in the whole sample; it was found that coercivity scales with the ordered phase volume fraction [34], therefore the resultant coercivity is also low.

\subsection{2 $\mathrm{Fe}_{3} \mathrm{Pt}$ films with different thickness}

Recalling the XRD patterns observed in bilayers and multilayers, this section aims to elucidate the structure of the $\mathrm{Fe}_{3} \mathrm{Pt}$ films. To study the relationship between orientation and thickness of the $\mathrm{Fe}_{3} \mathrm{Pt}$ films, films of $30 \mathrm{~nm}, 65 \mathrm{~nm}$ and $100 \mathrm{~nm}$ were deposited at the same potential and various times; the composition determined by EDS was within $75 \pm 2$ at. $\%$. The XRD patterns for the as-deposited and annealed films are shown in Fig.4.41. Similar to what has been discussed above, they all showed a BCC (110) texture in the as-deposited state; after annealing at $400^{\circ} \mathrm{C}$, a (200) peak was observed; the (200) peak separated into (200) and (002) upon $550^{\circ} \mathrm{C}$ annealing, while at the same time the original BCC (110) peak became weaker; after $650^{\circ} \mathrm{C}$ anneal, the BCC (110) completely disappeared; all films showed a stronger (002) to (200) texture; the $65 \mathrm{~nm}$ and $100 \mathrm{~nm}$ films, showed a peak at about $24.5^{\circ}$. This peak could not be assigned to the (100) peak of the ordered $\mathrm{L1}_{2} \mathrm{Fe}_{3} \mathrm{Pt}$, which should appear around $22.9^{\circ}$; meanwhile, $\mathrm{L1}_{2} \mathrm{Fe}_{3} \mathrm{Pt}$ will not show the separation of (200) into (200) and (002), since it has a cubic symmetry. The actual structure will be investigated in the following TEM section. 


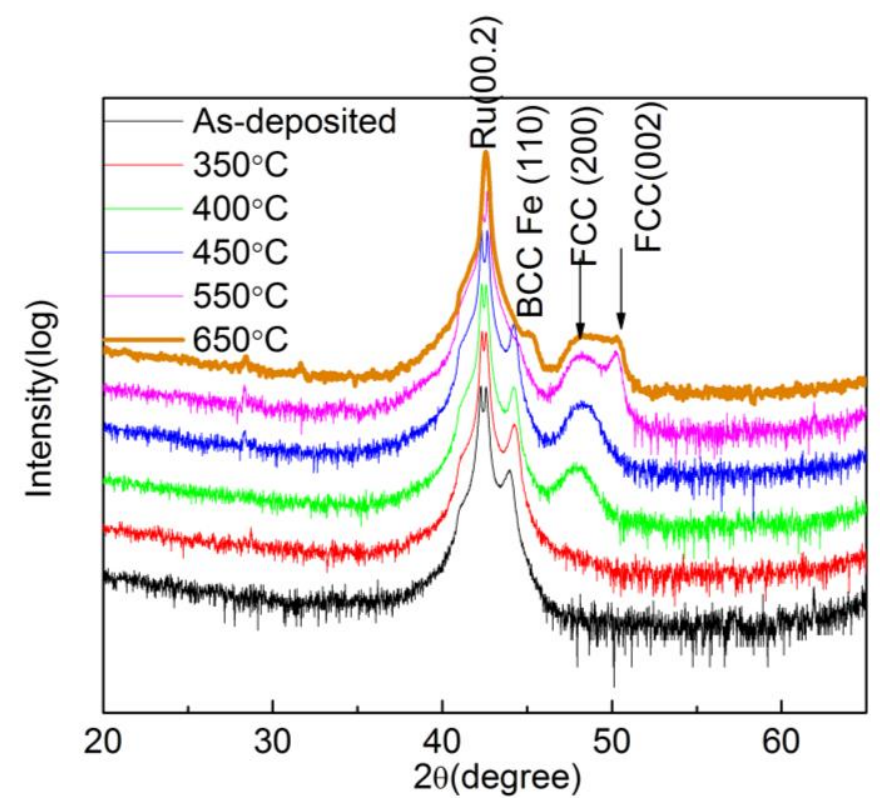

a)

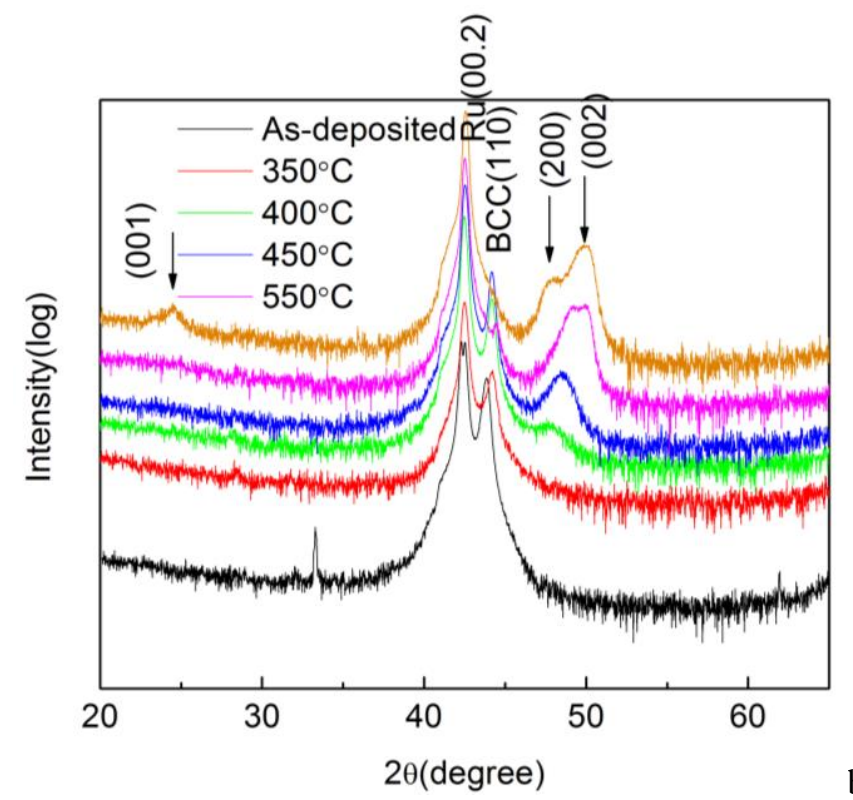

Figure 4.41 XRD patterns for $\mathrm{Fe}_{3} \mathrm{Pt}$ with different thickness after annealing at various temperatures: a) $30 \mathrm{~nm}$; b) $100 \mathrm{~nm}$. (Notice that the $65 \mathrm{~nm}$ one has been shown in Fig.4.38d)).

The magnetic loops for these films are shown in Fig.4.42. Relatively low coercivity was observed for all films at all temperatures, suggesting that no high anisotropy structure was obtained; in all films, however, the anisotropy greatly increased from $550^{\circ} \mathrm{C}$ annealing to $650^{\circ} \mathrm{C}$, as suggested by the about $6 \mathrm{kOe}$ larger saturation field. 

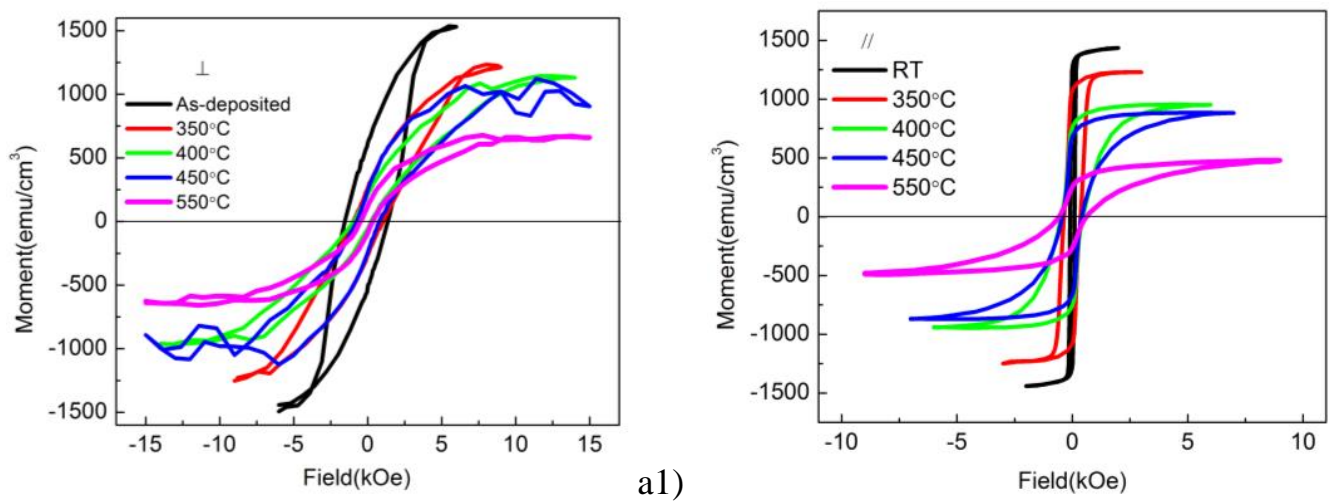

a2)
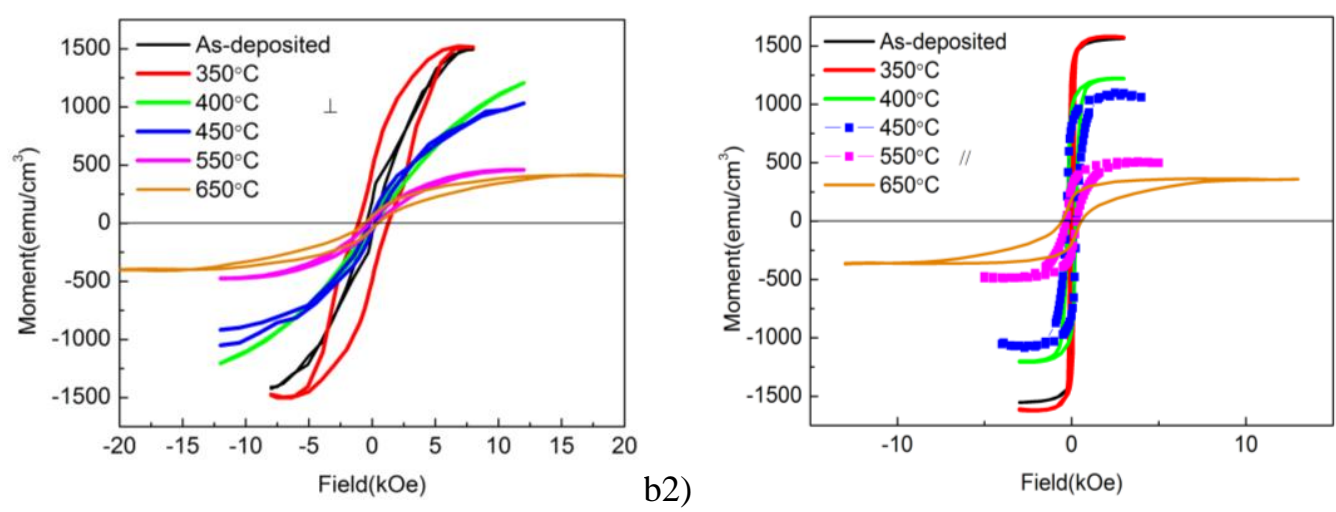

b2)

Figure 4.42 Out of plane and in- plane magnetic loops for $\mathrm{Fe}_{3} \mathrm{Pt}$ films with different thickness a1) $30 \mathrm{~nm} \perp$; a2) $30 \mathrm{~nm} / /$; b1) $100 \mathrm{~nm} \perp$; b2) $100 \mathrm{~nm} / /$.

For all thicknesses, a relatively large out of plane coercivity (1 3 kOe) was observed, both in as-deposited and after $350^{\circ} \mathrm{C}$ annealing (Fig.4.43).

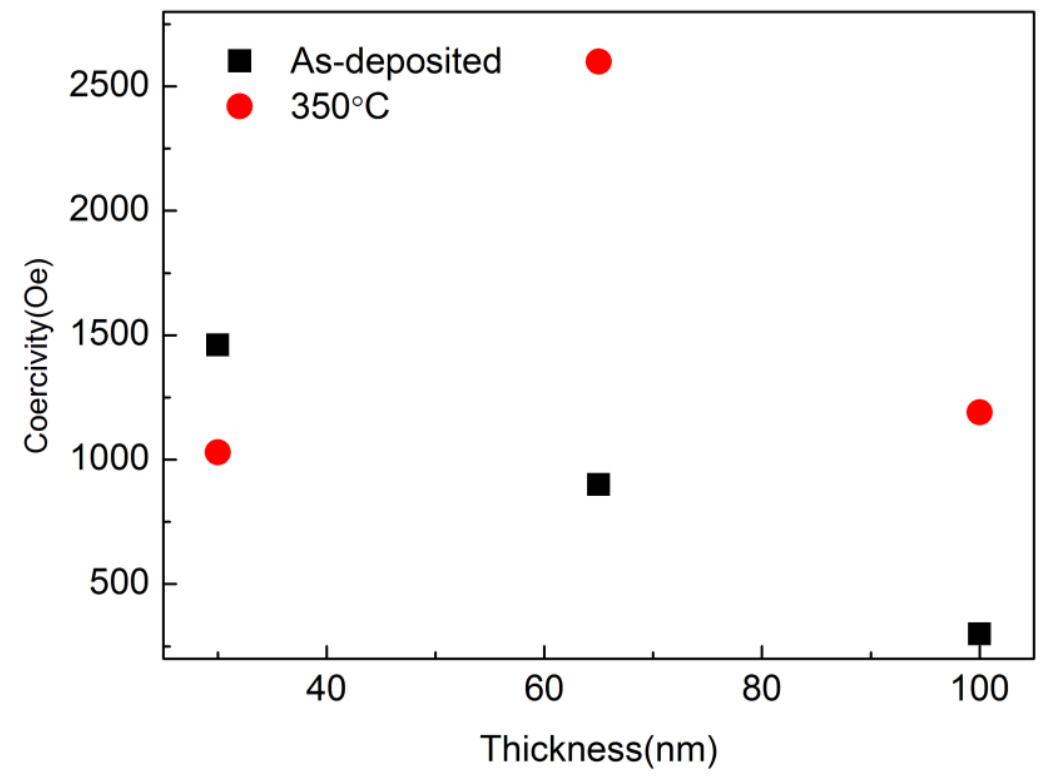

Figure 4.43 Perpendicular coercivity for the three $\mathrm{Fe}_{3} \mathrm{Pt}$ films as-deposited and after annealing at $350^{\circ} \mathrm{C}$. 


\subsubsection{Microstructure of $\mathrm{Fe}_{3} \mathrm{Pt}$}

As discussed in Section 4.5.2, there seems to be a FCC to $\mathrm{L}_{0}$ transition in $\mathrm{Fe}_{3} \mathrm{Pt}$ alloys, for which $\mathrm{L1}_{2}$ is the thermodynamically favored phase. The relatively small coercivity obtained in the annealed films, however, questions the existence of a $\mathrm{L}_{0}$ phase, since this should lead to quite a large coercivity. To elucidate the crystal structure in these films, HRTEM with nano-probe EDS was performed.

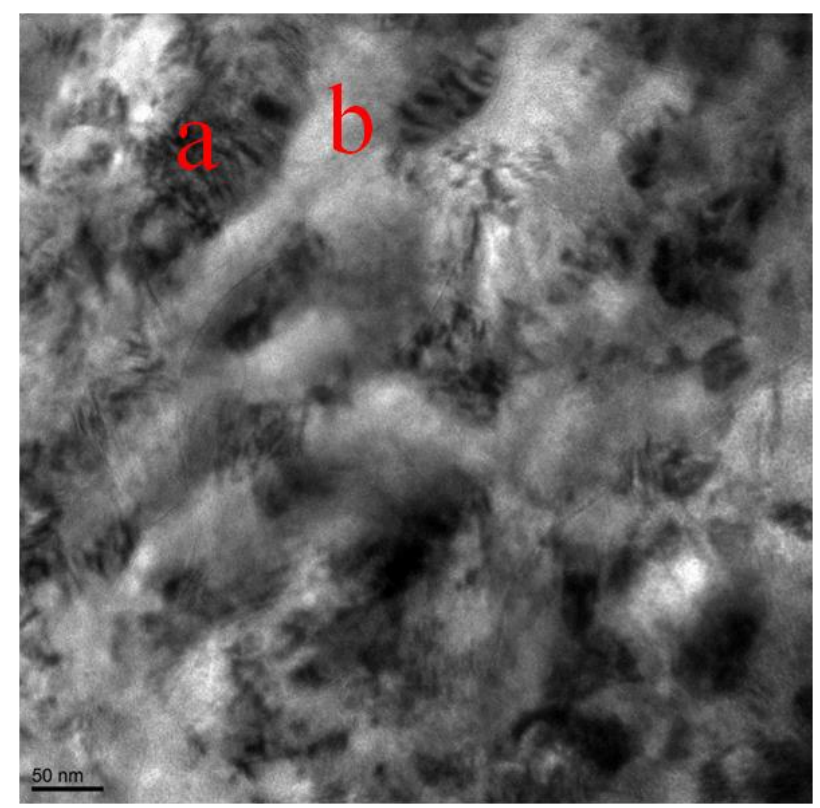

Figure 4.44. TEM image for $65 \mathrm{~nm} \mathrm{Fe} 3 \mathrm{Pt}_{3}$ film after annealing at $700^{\circ} \mathrm{C}$; nano-probe EDS on a and b showing Fe at.\% of 75 and 78 , respectively.

In Fig.4.44, a bright field TEM image for a $65 \mathrm{~nm}$ thick sample after annealing at $700^{\circ} \mathrm{C}$ is shown. Significant contrast was observed. Nano-probe EDS was performed on bright and dark areas in this image, which showed 75at.\% and 78at.\% of Fe, respectively, ruling out compositional gradient contributions to the contrast. SAED on the dark region is shown in Fig.4.45; superlattice diffraction from (110) and (112) reflections were clearly shown in the pattern, confirming the existence of a tetragonal phase. Together with the EDS data, it is concluded that a tetragonal phase with $~ 75$ at.\% of $\mathrm{Fe}$ was detected for $\mathrm{Fe}_{3} \mathrm{Pt}$ film after $700^{\circ} \mathrm{C}$ annealing. Lattice constant calculations give $\mathrm{a}=0.366 \mathrm{~nm}$ and $\mathrm{c}=0.377 \mathrm{~nm}$; in other words, the tetragonal phase has an expanded c axis instead of the usual contracted c-axis observed in the $\mathrm{L} 1_{0}$ phase. 


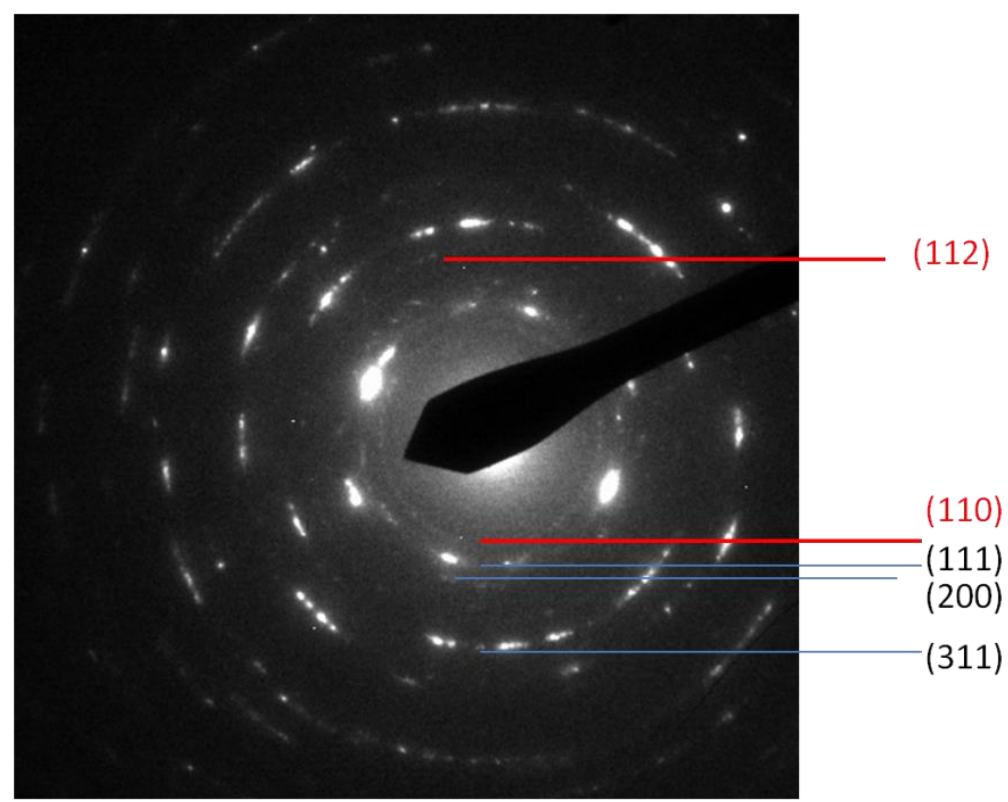

Figure 4.45 SAED on the dark region shown in Fig.4.44: the (110) and (112) superlattice diffraction are clearly shown.

Grains with $~ 75$ at.\% of Fe were imaged with HRTEM, shown in Fig.4.46. The interphase spacing was determined to be $0.261 \mathrm{~nm}$, which is close to the $0.259 \mathrm{~nm}$ using the two lattice constants determined above for the (110) orientation, further suggesting the existence of a tetragonal phase.

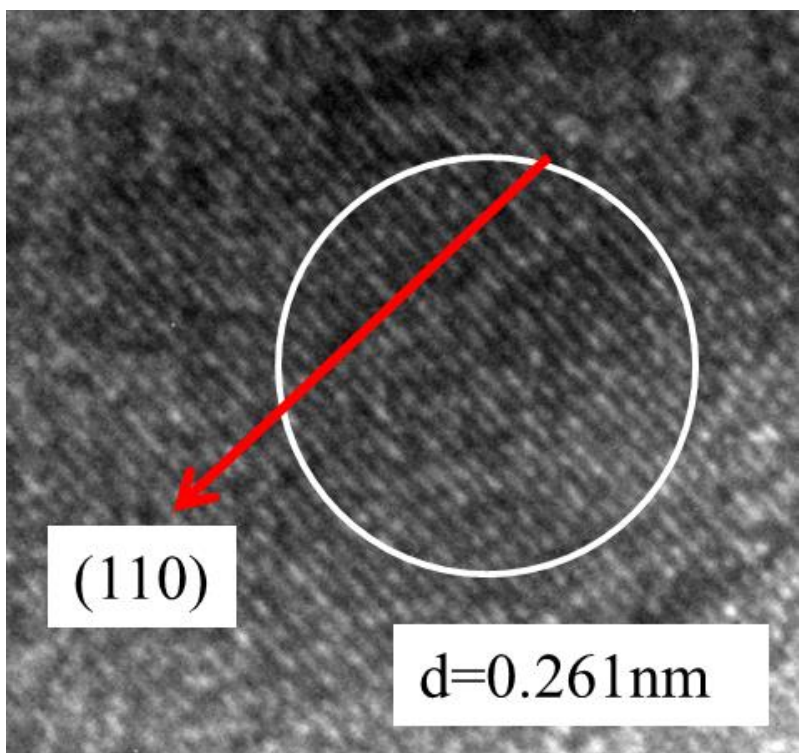

Figure 4.46 Grains with $~ 75$ at. $\%$ of $\mathrm{Fe}$, showing a lattice spacing of $0.261 \mathrm{~nm}$, close to the (110) spacing determined from diffraction pattern in Fig.4.45.

Recently, theoretical and experimental work by other groups shows that there may exist a martensitic transformation for $\mathrm{Fe}_{3} \mathrm{Pt}$ [45-47], leading to formation of a 
tetragonal structure where both $\mathrm{c} / \mathrm{a}>1$ and $\mathrm{c} / \mathrm{a}<1$ are possible. Yamamoto et al. [47] performed energy calculation with respect to the tetragonality c/a and the volume of the unit cell; they concluded that $\mathrm{L}_{2}$ is the ground state for the alloy; with variation of c/a, an increase in energy was observed with respect to the ground state, which, however, was small. A simple comparison with the c/a ratio in our study shows an increase in energy of $0.01 \mathrm{eV} / \mathrm{at}$ with a fixed unit cell of 352.9 a.u. ${ }^{3} /$ cell.

To summarize, the XRD patterns, TEM microstructure and SAED data shown above all suggest a $\mathrm{BCC}$ to $\mathrm{FCT}$ transition in $\mathrm{Fe}_{3} \mathrm{Pt}$ film: the as-deposited film shows a random BCC structure, whereas random FCC should be expected; after annealing, transformation to FCT occurs, where $\mathrm{L}_{2}$ is the thermodynamic stable phase. The unexpected phases and phase transformation here may be explained by a metastable phase diagram extrapolated from the thermodynamic equilibrium phase diagram [48], which is originated both by kinetic and thermodynamic effects. The phase diagram for Fe-Pt shown in Fig.1.13 was superposed with phase boundary extrapolated lines in the most simplified way, as shown in Fig.4.47. The extrapolation of region A indicates that random $\mathrm{BCC}$ can be a metastable structure for $\mathrm{Fe}_{3} \mathrm{Pt}$ at relatively low temperatures; aging below $\sim 800 \mathrm{~K}$ may lead to another metastable phase of $\mathrm{L}_{0}$, indicated by the intersection with region B extrapolated lines. Further aging at high temperatures should finally result in the thermodynamically stable $\mathrm{L}_{2}$ structure. 


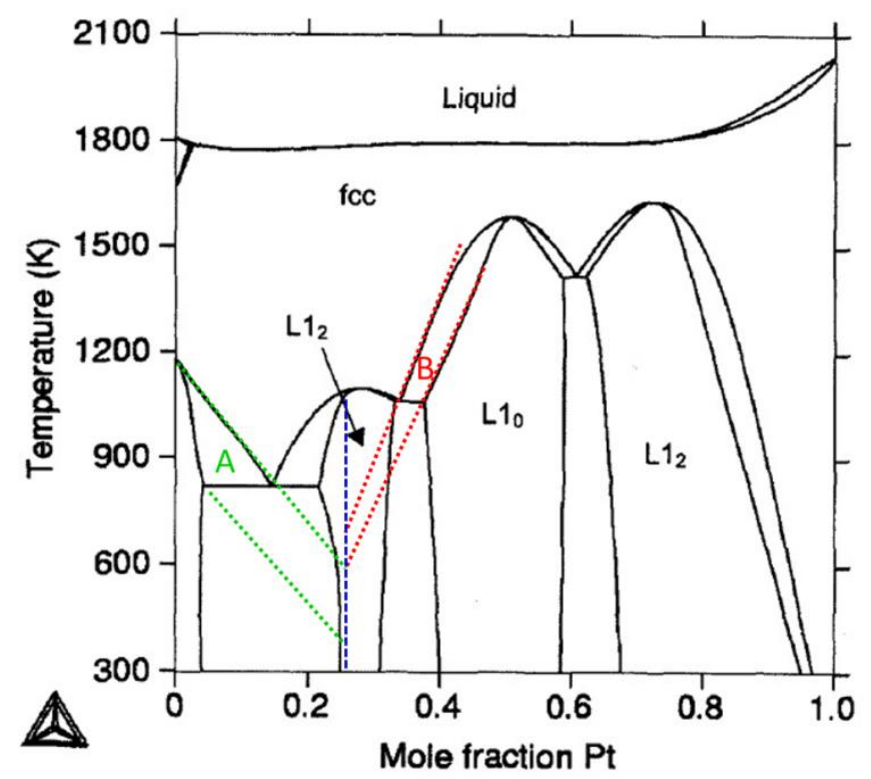

Figure 4.47 Metastable phase diagram for Fe-Pt. The blue vertical line corresponds to the composition of $\mathrm{Fe}_{3} \mathrm{Pt}$; $\mathrm{A}$ is a region for random $\mathrm{BCC}$ and $\mathrm{FCC}$, and region $\mathrm{B}$ for random $\mathrm{FCC}$ and $\mathrm{L} 1_{0}$.

Regarding the metastable phase diagram, the phase obtained is determined by both nucleation kinetics and thermodynamics. We hypothesize that a metastable BCC structured is obtained in the as-deposited films, due to the fact that its nucleation is more favored; upon annealing at relatively low temperatures, the metastable BCC slowly transforms to another metastable phase of $\mathrm{L} 1_{0}$. It should be noted that another intermediate FCC phase may form before transformation to $\mathrm{L}_{0}$; as we can see from Fig.4.41, there exists a single FCC (200) peak before separation into (200) and (002) between $400^{\circ} \mathrm{C}$ and $450^{\circ} \mathrm{C}$. Further annealing at relatively high temperatures leads to the complete disappearance of the BCC phase.

The phase transformation for $\mathrm{Fe}_{3} \mathrm{Pt}$ requires further study, partially due to the inconsistency in the available literature. Both FCC [49] and BCC [50] phases were observed in as-deposited films, while the phase after annealing is greatly dependent on the annealing process [49], e.g., in-situ or post -annealing. In their rf magnetron sputtered films, Hsiao et al. found that in-situ annealing leads to the formation of metastable FCT with coercivity $2.4 \mathrm{kOe}$, while post-annealing results in the $\mathrm{L}_{2}$ phase with lower coercivity [49]; the saturation magnetization also depends on the phase, 
FCT with lower $M_{s}$ and $L_{2}$ with higher $M_{s}$ [49]. More recently, Hsiao et al. reported a two-stage phase transformation process of BCC to disordered FCC, and then disordered FCC to $\mathrm{L1}_{2}[50]$.

As can be seen in Fig.4.43, the saturation magnetization for $\mathrm{Fe}_{3} \mathrm{Pt}$ films decreases with increasing annealing temperatures; the reason for it is not clear. Magnetization is determined by the distance between spins via exchange coupling, and is therefore sensitive to the atomic configuration. Different magnetization for the same composition is due to different crystal structures, as discussed above for $\mathrm{L}_{2}$ and FCT. On the other hand, a rough approximation for the alloy magnetization can be made by assuming a Vegards'law behavior, which gives a magnetization of $1285 \mathrm{emu} / \mathrm{cm}^{3}$, comparing with $\sim 500 \mathrm{emu} / \mathrm{cm}^{3}$ after $700^{\circ} \mathrm{C}$ annealing.

To finally summarize, the phase we observed in our films could be FCT, or a mixture of $\mathrm{FCC}$ and $\mathrm{L1}_{2}$

\subsection{Conclusion}

A novel alkaline solution has been developed for the electrodeposition of Fe-Pt. Oxygen content in the deposits was greatly reduced, and smooth morphology was obtained in the deposits. As-deposited near equiatomic films were magnetically soft with random FCC structures. Upon annealing, films started to harden at various temperatures, which greatly depends on the thickness of the films. Ordering occurred at lower temperatures for thicker films. For $\sim 100 \mathrm{~nm}$ thick films, hardening started at $350^{\circ} \mathrm{C}$, and at $400^{\circ} \mathrm{C}$, coercivity of $6 \mathrm{kOe}$ was observed. After annealing at $450^{\circ} \mathrm{C}$, hardening was already maximized, with coercivity of $\sim 13 \mathrm{kOe}$. Bilayer with $15 \mathrm{~nm}$ near equiatomic FePt and different thicknesses of Fe rich $\mathrm{Fe}_{3} \mathrm{Pt}(5 \mathrm{~nm}$ to $50 \mathrm{~nm}$ ) was deposited, and it was found that by coupling an appropriate thickness of Fe rich layer (here $5 \mathrm{~nm}$ or $10 \mathrm{~nm}$ ), the hardening process can be enhanced with about $50 \%$ of coercivity increase comparing with the single $\mathrm{FePt}$ layer after annealing to $550^{\circ} \mathrm{C}$ and 
$650^{\circ} \mathrm{C}$. Multilayers with two different configurations were also constructed; it was found that the enhancement in coercivity is roughly independent of the layer configuration. Therefore, the bilayer structure is sufficient to enhance coercivity while keeping the total thickness of the whole structure within 20-30 nm. Microstructure by TEM showed $\sim 5 \mathrm{~nm}$ grain size for the as-deposited equiatomic FePt films, and it increased to $\sim 25 \mathrm{~nm}$ after annealing at $700^{\circ} \mathrm{C}$. Fe rich films with $56 \%$ to $75 \%$ of $\mathrm{Fe}$ showed an increasing BCC (110) texture; after annealing, the (110) texture disappeared and FCC (200) appeared and separated into (200) and (002) with increasing annealing temperature. As-deposited or thermally annealed $\left(350^{\circ} \mathrm{C}\right) \mathrm{Fe}_{3} \mathrm{Pt}$ films of 30- $100 \mathrm{~nm}$ showed an out of plane coercivity of 500 to 2500 Oe. SAED suggested the existence of an expanded c-axis FCT structure after annealing to $700^{\circ} \mathrm{C}$. 


\section{References}

[1] O. Gutfleisch, J. Lyubina, K.-H. MÜller, L. Schultz, Adv. Eng. Mater. 7 (2005) 208.

[2] S. Sun, Adv. Mater. 18 (2006) 393-403.

[3] S. Jeong, T. Ohkubo, A.G. Roy, D.E. Laughlin, M.E. McHenry, J. Appl. Phys. 91 (2002) 6863

[4] S. Sun, Science 287 (2000) 1989.

[5] J.J. Mallett, E.B. Svedberg, S. Sayan, a. J. Shapiro, L. Wielunski, T.E. Madey, W.F. Egelhoff, T.P. Moffat, Electrochem. Solid-State Lett. 7 (2004) C121.

[6] K. Leistner, S. Oswald, J. Thomas, S. Fahler, H. Schlorb, L. Schultz, Electrochimi. Acta 52 (2006) 194.

[7] T. Itoh, T. Kato, S. Iwata, S. Tsunashima, IEEE Trans. Magn. 41 (2005) 3217.

[8] M. Datta, Electrochimi. Acta 45 (2000) 2535.

[9] F.M.F. Rhen, J.M.D. Coey, J. Magn. Magn. Mater. 322 (2010) 1572.

[10] K. Leistner, P. Schaaf, A. Voss, S. Fahler, L. Schultz, H. Schlorb, Electrochimi. Acta 53 (2008) 6973.

[11] K. Leistner, S. Fähler, H. Schlörb, L. Schultz, Electrochem. Commun. 8 (2006) 916.

[12] S.R. Brankovic, S.-E. Bae, D. Litvinov, Electrochimi. Acta 53 (2008) 5934.

[13] D. Liang, J.J. Mallett, G. Zangari, ACS Appl. Mater. Interfaces 2 (2010) 961.

[14] B.M.E. Baumgartner, C.J. Raub, Platinum Metals Rev. (1988) 188.

[15] X. Xu, G. Zangari, J. Electrochem. Soc 155 (2008) D742.

[16] A.J. Gregory, W. Levason, D. Pletcher, J. Electroanal. Chem. 348 (1993) 211.

[17] O. Berkh, Y. Shacham-Diamand, E. Gileadi, J. Appl. Electrochem. 38 (2008) 1275 .

[18] T.P. Moffat, J.J. Mallett, S.-M. Hwang, J. Electrochem. Soc 156 (2009) B238. 
[19] G. Karlberg, T. Jaramillo, E. Skúlason, J. Rossmeis1, T. Bligaard, J. Nørskov, Phys. Rev. Lett. 99 (2007) 126101.

[20] G. Brauer, ed., Handbook of Preparative Inorganic Chemistry, 2nd ed., Academic Press New York, London, 1965.

[21] D. Liang, J.J. Mallett, G. Zangari, J. Electrochem. Soc. 158 (2011) D149.

[22] L. Sillen, L. G., Gunnar, Stability Constants of Metal-Ion Complexes, 2nd Ed.., 1964.

[23] L.C. Königsberger, E. Königsberger, P.M. May, G.T. Hefter, J. Inorg. Biochem. 78 (2000) 175.

[24] V. Cuculić, I. Pižeta, M. Branica, J. Electroanal. Chem. 583 (2005) 140.

[25] K. Micskei, J. Chem. Soc., Dalton Trans. (1987) 255.

[26] K. Leistner, A. Krause, S. Fähler, H. Schlörb, L. Schultz, Electrochimi. Acta Acta 52 (2006) 170.

[27] H.K. Hardy, Acta Metall. 1 (1953) 202.

[28] J.J. Mallett, E.B. Svedberg, J.E. Bonevich, A.J. Shapiro, W.F. Egelhoff, T.P. Moffat, J. Electrochem. Soc 155 (2008) D1.

[29] D. Liang, J.J. Mallett, G. Zangari, Electrochimi. Acta 55 (2010) 8100.

[30] H. Okamoto, in:, Phase Diagram ASM International, Vol. 9,1st, 1993, p. 330.

[31] A. Patterson, Phys. Rev. 56 (1939) 978.

[32] J. Wan, M.J. Bonder, Y. Huang, G.C. Hadjipanayis, C. Ni, J. Magn. Magn. Mater. 322 (2010) 1811.

[33] J.S. Jiang, J.E. Pearson, Z.Y. Liu, B. Kabius, S. Trasobares, D.J. Miller, S.D. Bader, D.R. Lee, D. Haskel, G. Srajer, J.P. Liu, J. Appl. Phys. 97 (2005) $10 \mathrm{~K} 311$.

[34] R. a. Ristau, K. Barmak, L.H. Lewis, K.R. Coffey, J.K. Howard, J. Appl. Phys. 86 (1999) 4527.

[35] S. Jeong, M.E. McHenry, D.E. Laughlin, IEEE Trans. Magn. 37 (2001) 1309.

[36] K. Barmak, J. Kim, S. Shell, E.B. Svedberg, J.K. Howard, Appl. Phys. Lett. 80 (2002) 4268. 
[37] Y. Tamada, S. Yamamoto, M. Takano, S. Nasu, T. Ono, Phys. Status Solidi C 4 (2007) 4503.

[38] F. Casoli, F. Albertini, L. Nasi, S. Fabbrici, R. Cabassi, F. Bolzoni, C. Bocchi, Appl. Phys. Lett. 92 (2008) 142506.

[39] J.-L. Tsai, H.-T. Tzeng, B.-F. Liu, Thin Solid Films 518 (2010) 7271-7274.

[40] Y. Endo, N. Kikuchi, O. Kitakami, Y. Shimada, J. Appl. Phys. 89 (2001) 7065.

[41] D.C. Berry, K. Barmak, J. Appl. Phys. 101 (2007) 014905.

[42] R. Skomski, G.C. Hadjipanayis, D.J. Sellmyer, IEEE Trans. Magn. 38 (2002) 2802-2804.

[43] Z.L. Wang, J.P. Liu, IEEE Trans. Magn. 38 (2002) 2598.

[44] L. Callegaro, E. Puppin, P.L. Cavallotti, G. Zangari, J. Magn. Magn. Mater. 155 (1996) 190.

[45] T. Kakeshita, T. Takeuchi, T. Fukuda, M. Tsujiguchi, T. Saburi, R. Oshima, S. Muto, Appl. Phys. Lett. 77 (2000) 1502.

[46] M. Yamamoto, S. Sekida, T. Fukuda, T. Kakeshita, K. Takahashi, K. Koyama, H. Nojiri, J. Alloys Compd. 509 (2011) 8530.

[47] T. Yamamoto, M. Yamamoto, T. Fukuda, T. Kakeshita, H. Akai, Mater. Trans. $51(2010) 896$.

[48] L. Battezzati, C. Antonione, M. Baricco, J. Alloys Compd. 247 (1997) 164.

[49] S.N. Hsiao, S.K. Chen, Y.W. Hsu, F.T. Yuan, H.W. Huang, T.S. Chin, W.C. Chang, H.Y. Lee, IEEE Trans. Magn. 44 (2008) 3902-3905.

[50] S.N. Hsiao, S.K. Chen, S.H. Liu, H.Y. Lee, J. Appl. Phys. 111 (2012) 07A313. 


\section{Chapter 5 Electrodeposition of immiscible Au-Ni alloys}

\section{Overview}

Au-Ni alloys have been recently extensively researched due to their unique combination of physical, chemical and mechanical properties and therefore potential applications in electrical contact and switching materials [1], magnetic drug delivery [2] and catalysts for $\mathrm{CO}$ oxidation [3]. Thermodynamically, it is also interesting for its ordering and clustering behaviors at different temperatures [4]. Bulk Au-Ni can form face centered cubic (FCC) solid solutions in the whole compositional range above $\sim 1090 \mathrm{~K}$; at lower temperatures, however, there is a large immiscible gap resulting in phase separated FCC Au and FCC Ni[5]. It was also shown that Au-Ni surface alloys exist within the immiscible gap, due to the increasing coordination numbers of the surface $\mathrm{Ni}$ induced by the larger electron density of $\mathrm{Au}[6]$.

Electrodeposition enables to tune the driving force for film formation, for example, far away from thermodynamic equilibrium, making it possible to synthesize structures different from those predicted in equilibrium phase diagrams; experimentally, it has been shown that ordered or metastable structures absent for bulk equilibrium phase diagrams could be obtained [7]. For the target system here, Au-Ni, Yamachika et al. showed that amorphous Au-Ni alloys films could be electrodeposited from a gold cyanide and nickel sulfate solution[8]; Rouya et al. showed nanocrystalline/ amorphous structures for the whole composition range from a gold sulfite solution[9].

In this study, gold chloride, which is less strongly complexed than either gold cyanide or gold sulfite, was used; nickel sulfate without any complexing agent was the $\mathrm{Ni}$ source, since this system is electrochemically more reversible. The electrochemical deposition process was analyzed with the UPCD model; the structures of the deposited phases were studied using XRD, XPS and HRTEM. 


\subsection{Experimental}

The solutions listed in Table 5.1 were used for the experiments. Three different concentrations of $\mathrm{NiSO}_{4}$ were chosen; solutions A-D were only for EQCM measurement for single metals; in order for all solutions to have similar conductivity, $0.5 \mathrm{M} \mathrm{Na}_{2} \mathrm{SO}_{4}$ was added as supporting electrolyte for some solutions with $\mathrm{Ni}$ less than 0.5M; solutions 1-3 were used for the potentiostatic deposition of alloys.

Au-Ni films were electrodeposited at constant potential from the three electrolytes reported in Table 5.1, onto $100 \mathrm{~nm}$ thick unoriented Ruthenium or 100nm Cu (111) layers, sputter deposited onto a $5 \mathrm{~nm}$ Ta adhesion layer on top of the native oxide of Si (001) wafers. Film growth experiments were performed using a three-electrode cylinder cell in the vertical configuration. The counter-electrode was a Pt mesh, and the reference was a saturated calomel electrode (SCE). In the following all potentials will be referred to SCE $\left(E_{S C E}^{0}=0.241 \mathrm{~V}_{\mathrm{SHE}}\right)$. The $\mathrm{pH}$ of all solutions was adjusted to 2.5 by addition of diluted $\mathrm{NaOH}$ or $\mathrm{H}_{2} \mathrm{SO}_{4}$.

All solutions for EQCM characterization were dearated by purging nitrogen gas for about 30 min before measurement, while very gentle flow of nitrogen was kept during experiments; all the cyclic voltammetry $(\mathrm{CV})$ curves were collected at a scan rate of $5 \mathrm{mV} / \mathrm{s}$.

Alloy composition was determined by energy dispersive spectroscopy EDS using a detector attached to a JEOL JSM-6700F scanning electron microscope (SEM). The ratio $\mathrm{Ni}$ to $\mathrm{Au}$ content was determined using an accelerating voltage of $20 \mathrm{kV}$ and the $\mathrm{K}$ line for $\mathrm{Ni}$, the $\mathrm{M}$ line for $\mathrm{Au}$. Selected samples were also determined by X-ray fluorescence (XRF, in ESRF Grenoble, France), which confirms a very good match of the compositions measured by EDS and XRF. Morphology was obtained with the above SEM. Crystal structure was determined by X-ray diffraction, using a Scintag XDS 2000 diffractometer with $\mathrm{Cu} \mathrm{K} \alpha$ radiation $(\lambda=1.5418 \AA)$ in the $\theta / 2 \theta$ geometry. Glancing angle XRD (GAXRD) was performed for selected films in ESRF, with XRD 
glancing angle (0.5 degrees incidence angle), acceleration voltage of $15 \mathrm{keV}, 1$ $\lambda=0.8267 \AA$, and beam area of $0.2 \times 0.02 \mathrm{~mm}$. Film thickness was estimated by EQCM data and composition data, and confimred by using an optical profilometer (Veeco Wyto NT1100).

Chemical states of $\mathrm{Au}$ and $\mathrm{Ni}$ elements in the deposits were determined using hard X-ray photoelectron spectroscopy (HXPES) in European Synchrotron Radiation Facility (ESRF Grenoble, France) with the help of Dr. Zegenhagen. HXPES was performed using the SPECS Phoibos $225 \mathrm{HV}$ electron analyzer at $3 \mathrm{keV}$ (exactly 2.98 $\mathrm{keV}$ ) and $6 \mathrm{keV}$ (exactly 5.950) $\mathrm{keV} \mathrm{X}$-ray excitation energy at ID32 beamline of the ESRF. The HAXPES spectra were recorded in fixed analyser transmission mode (30 $\mathrm{eV}$ pass energy and exit slit size of $3 \times 20 \mathrm{~mm}$ giving about $50 \mathrm{meV}$ energy resolution). The overall energy resolution was about $0.5 \mathrm{eV}$ and $1.0 \mathrm{eV}$ at 3 and $6 \mathrm{keV}$, respectively. The take-off angle between sample surface normal and electron analyser, called $\alpha$ was kept at $45^{\circ}$. The binding energy was calibrated before each sample measurement with clean $\mathrm{Au}$ foil measurement using the $4 \mathrm{f}_{7 / 2}$ line position at $84.0 \mathrm{eV}$. HAXPES measurement was performed at two different incidence energies to distinguish between surface and bulk chemical properties. The inelastic mean free path (IMFP) is proportional to the square root of the electron kinetic energy [10]. At 3 $\mathrm{keV}$ photon energy, IMFP $\sim 3.5 \mathrm{~nm}$ while at $6 \mathrm{keV}$ photon energy IMFP $\sim 5 \mathrm{~nm}$ [11]. Thus at $6 \mathrm{keV}$ the surface contribution of the signal is reduced

Microstructure of deposits was characterized by high resolution TEM (HRTEM, Titan 80-300). The samples for TEM characterization were deposited onto $50 \mu \mathrm{m}$ thick $\mathrm{Cu}$ sheet; the samples were then thinned from the backside using ion beam milling (Gatan, Model 691) to obtain foils sufficiently thin for electron beams. During the process, liquid nitrogen was used to cool the sample, aiming to avoiding possible milling and heat induced artifacts.

Samples were analyzed usually within one day for XRD and EDS to reduce the possibility of phase evolution with time after deposition. However, this was not 
possible for TEM and XPS measurement, due to the fact that the former is time consuming and the latter was performed in Europe and samples were sent by mail. TEM samples were analyzed one year after the first time analysis, which showed similar structures. However, it is still possible that the actual phase structure is different from what is shown here.

Table 5.1 Formulation of the electrolyte used for experiments.

\begin{tabular}{ccccc}
\hline Solution & $\mathrm{HAuCl}_{4}$ & $\mathrm{NiSO}_{4}$ & $\mathrm{Na}_{2} \mathrm{SO}_{4}$ & $\mathrm{pH}$ \\
\hline$A$ & $0.5 m M$ & & $0.5 M$ & 2.5 \\
$B$ & - & $5 m M$ & $0.5 M$ & 2.5 \\
$C$ & - & $50 m M$ & $0.5 M$ & 2.5 \\
$D$ & - & $0.5 M$ & - & 2.5 \\
1 & $0.5 m M$ & $5 m M$ & $0.5 M$ & 2.5 \\
2 & $0.5 m M$ & $50 m M$ & $0.5 M$ & 2.5 \\
3 & $0.5 m M$ & $0.5 M$ & - & 2.5
\end{tabular}

\subsection{Electrodeposition of Au-Ni alloys}

\subsubsection{Electrochemical studies}

The $\mathrm{CV}$ and EQCM data for $0.5 \mathrm{mM} \mathrm{HAuCl}{ }_{4}$ with the $0.5 \mathrm{M} \mathrm{Na} \mathrm{NO}_{4}$ supporting electrolyte is displayed in Fig. 5.1. In Fig. 5.1 a), the mass change as a function of potential during scan was linear; this is a typical feature for deposition under diffusion limited control. This is expected as gold chloride is a weakly complexed compound and it starts depositing as positive as 


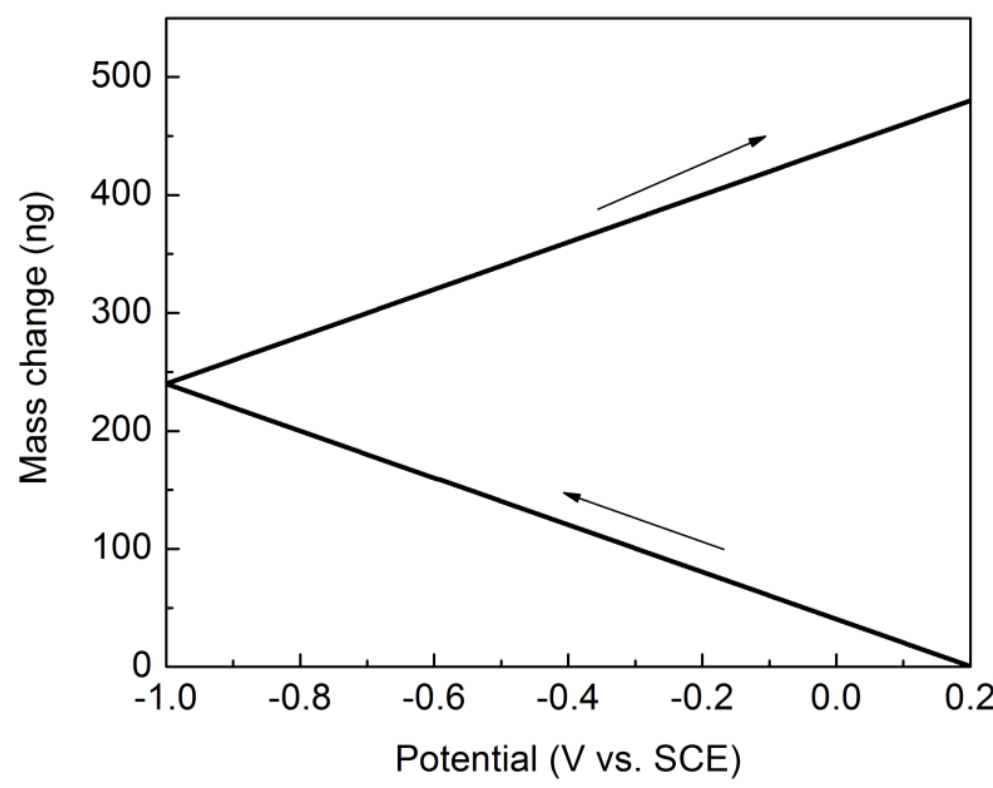

a)

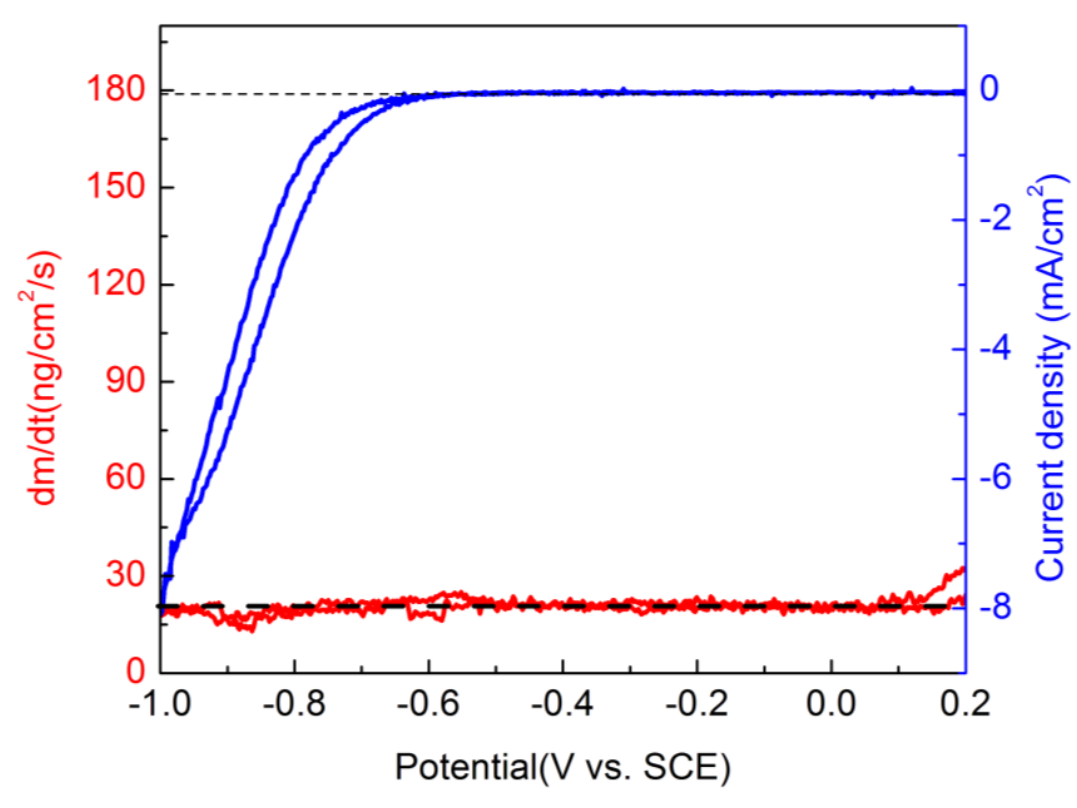

b)

Figure 5.1 a)Mass change vs. potential and b) $\mathrm{dm} / \mathrm{dt}$ and current density vs. potential for $0.5 \mathrm{mM}$ $\mathrm{HAuCl}_{4}+0.5 \mathrm{M} \mathrm{Na}_{2} \mathrm{SO}_{4} \mathrm{pH} 2.5$ solution; scan rate of $5 \mathrm{mV} / \mathrm{s}$.

$0.623 \mathrm{~V}_{\text {SCE }}$. The potential range used here is well within the diffusion limited control region for $\mathrm{Au}$ deposition. The electrochemical behavior is even better shown when differentiating the mass change with time and combined with $\mathrm{CV}$ data, as shown in Fig.5.1 b). The current density at potential more positive than $-0.43 \mathrm{~V}$ was about $0.038 \mathrm{~mA} / \mathrm{cm}^{2}$; this could be attributed to the diffusion limited current for $\mathrm{Au}$, and it corresponds to $\sim 26 \mathrm{ng} / \mathrm{cm}^{2} / \mathrm{s}$ of $\mathrm{Au}$ deposition assuming $100 \%$ efficiency, which is reasonable for a dearated solution before HER starts. The mass change determined 
from $\mathrm{dm} / \mathrm{dt}$ data is $\sim 22 \mathrm{ng} / \mathrm{cm}^{2} / \mathrm{s}$ and is in good agreement with the value calculated by current. The actual current efficiency was calculated according to the $\mathrm{CV}$ and $\mathrm{dm} / \mathrm{dt}$ data in Fig.5.1, as shown in Fig.5.2. Efficiency of $\sim 85 \%$ is obtained in the beginning, and it slowly drops upon HER to nearly $0 \%$ at $-1.0 \mathrm{~V}$.

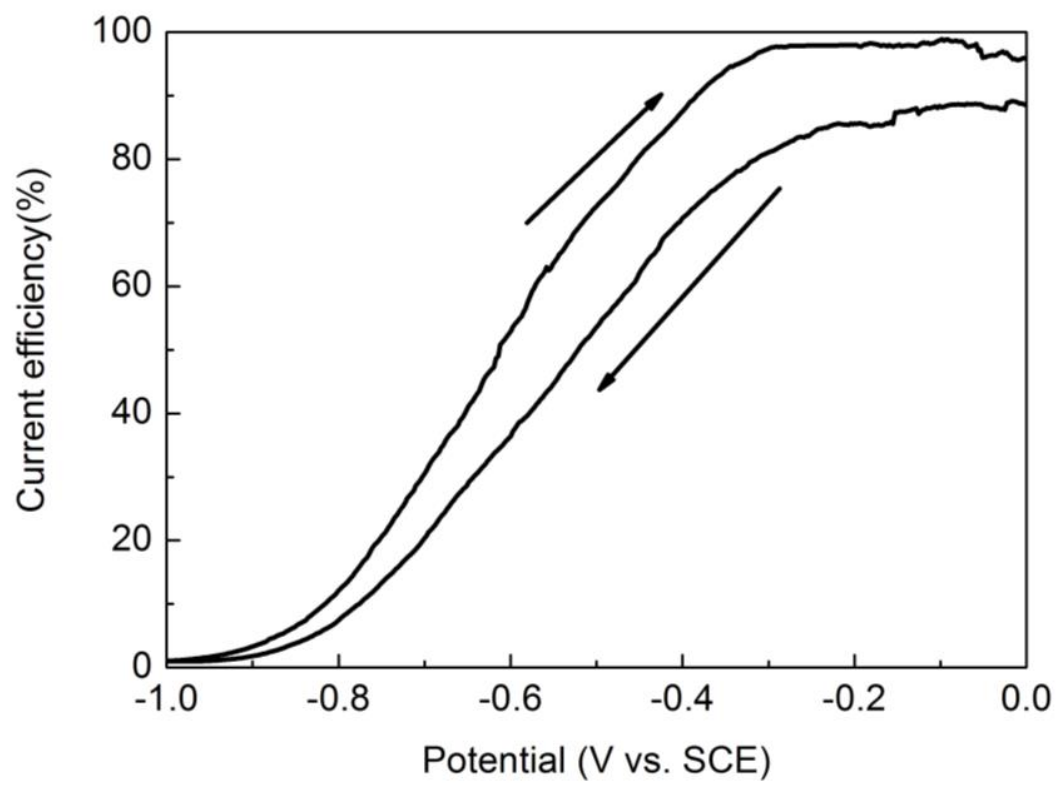

Figure 5.2 Current efficiency for $0.5 \mathrm{~m} \mathrm{HAuCl}_{4}+0.5 \mathrm{M} \mathrm{Na}_{2} \mathrm{SO}_{4} \mathrm{pH} 2.5$ solution extracted from data shown in Fig.5.1.

The $\mathrm{CV}$ and EQCM data for the $0.5 \mathrm{M} \mathrm{NiSO}$ solution are shown in Fig.5.3. Ni deposition started at $\sim-0.620 \mathrm{~V}$, comparing with $-0.5 \mathrm{~V}$ by Nernst' Equation. On the reverse scan, dissolution of $\mathrm{Ni}$ started at $-0.41 \mathrm{~V}$. As a result, this is a gap of $\sim 210 \mathrm{mV}$ between onset of deposition and dissolution, indicating their reversible characteristics of Ni electrodeposition. 


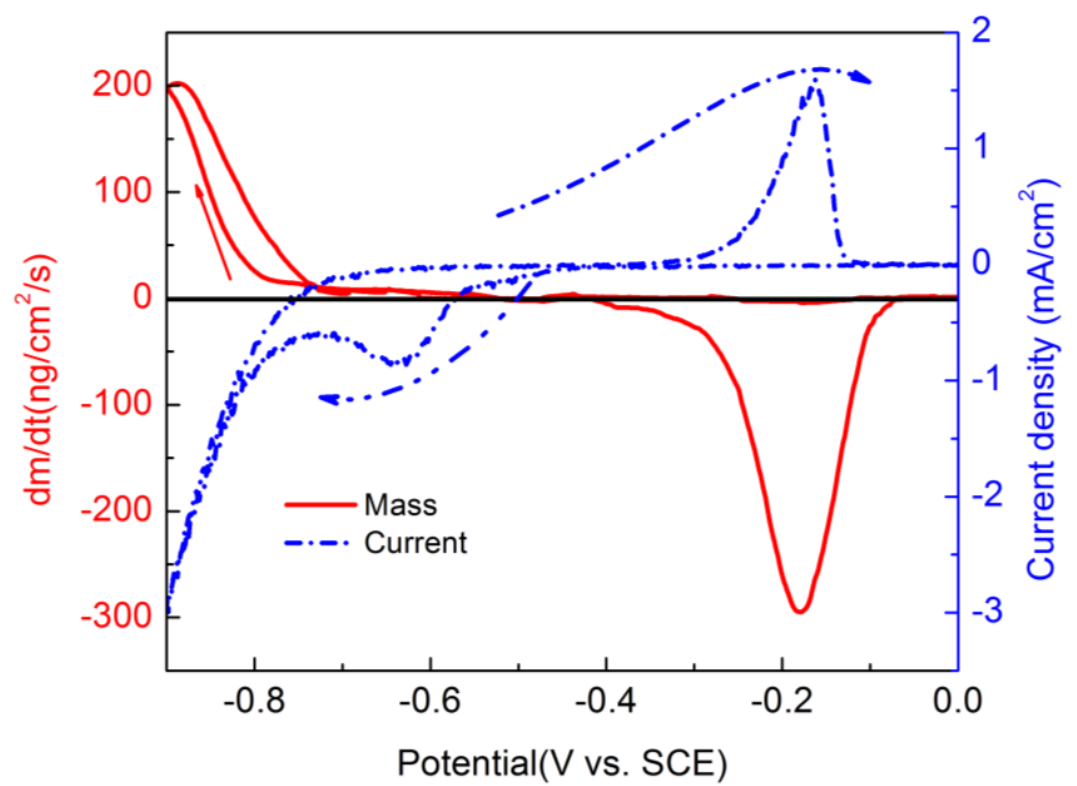

Figure $5.3 \mathrm{dm} / \mathrm{dt}$ and current density vs. potential for $0.5 \mathrm{M} \mathrm{NiSO}_{4}, \mathrm{pH} 2.5$ solution; scan rate of $5 \mathrm{mV} / \mathrm{s}$.

The corresponding data for the alloy deposition solution of $0.5 \mathrm{mM} \mathrm{HAuCl}{ }_{4}+0.5 \mathrm{M}$ $\mathrm{NiSO}_{4}$ are shown in Fig.5.4. In Fig.5.4 a), the mass change data showed a linear increase in mass until $\sim-0.5 \mathrm{~V}$, which can be attributed to the diffusion limited deposition of $\mathrm{Au}$, as indicated by the Au only solution in Fig.5.1; then mass increased rapidly as scan went more negative, which is due to the co-deposition of $\mathrm{Ni}$ with $\mathrm{Au}$. Combining CV and dm/dt data, the electrochemical behavior is more clearly shown in Fig. 5.4 b). The plateau of $\sim 21 \mathrm{ng} / \mathrm{cm}^{2} / \mathrm{s}$ until $-0.53 \mathrm{~V}$ in the $\mathrm{dm} / \mathrm{dt}$ data is very close to that from only Au solution, confirming the Au diffusion deposition in this potential range. 

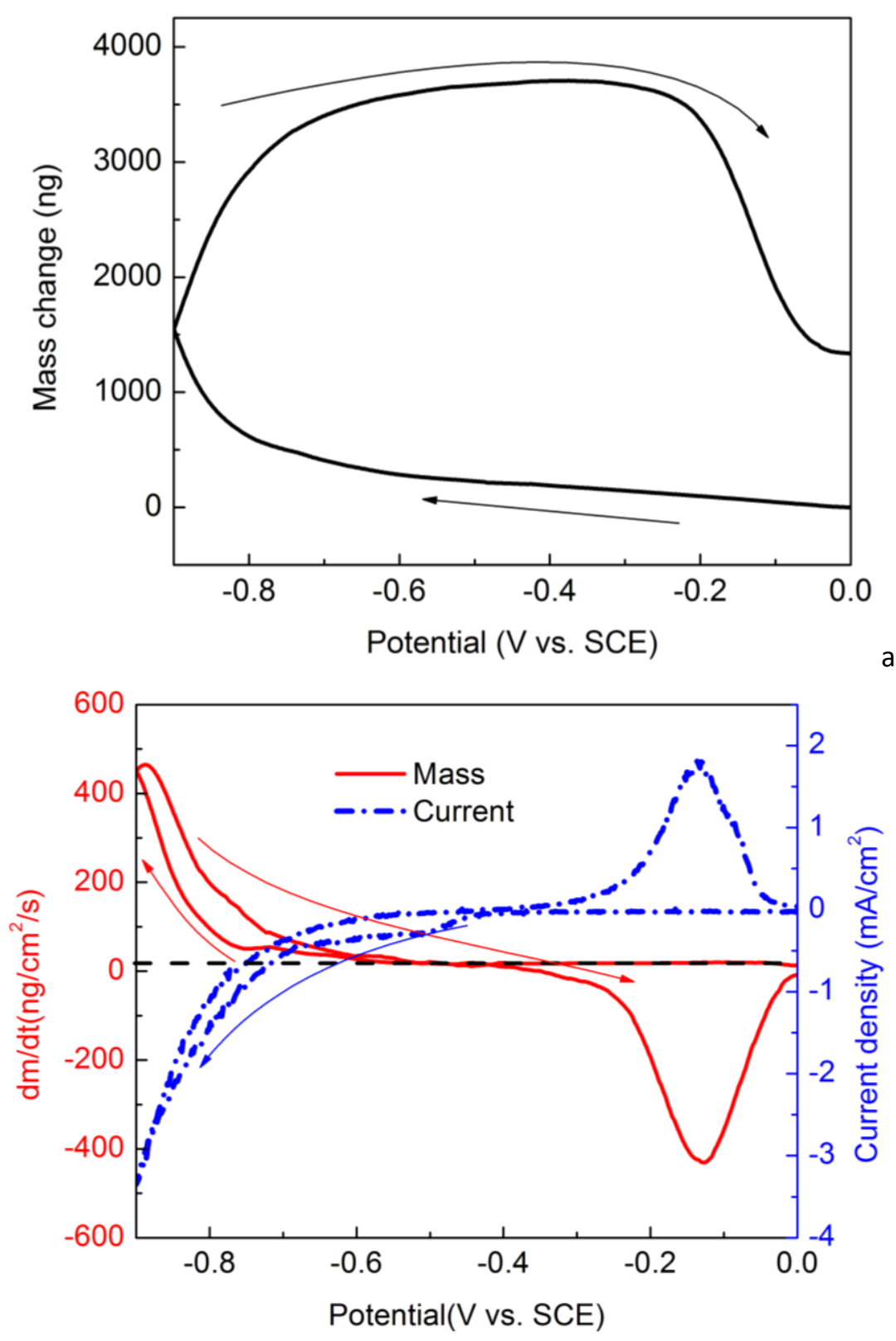

b)

Figure 5.4 a)Mass change vs. potential and b) $\mathrm{dm} / \mathrm{dt}$ and current density vs. potential for $0.5 \mathrm{mM}$ $\mathrm{HAuCl}_{4}+0.5 \mathrm{M} \mathrm{Na}_{2} \mathrm{SO}_{4} \mathrm{pH} 2.5$ solution; scan rate of $5 \mathrm{mV} / \mathrm{s}$. 


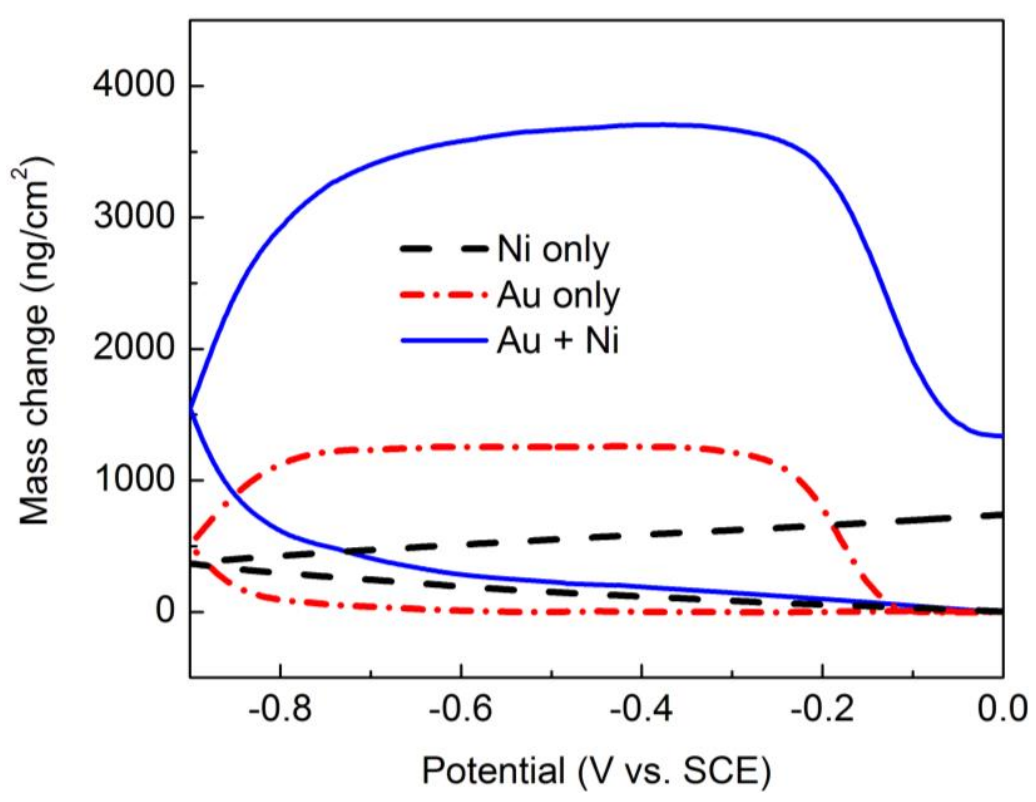

a)

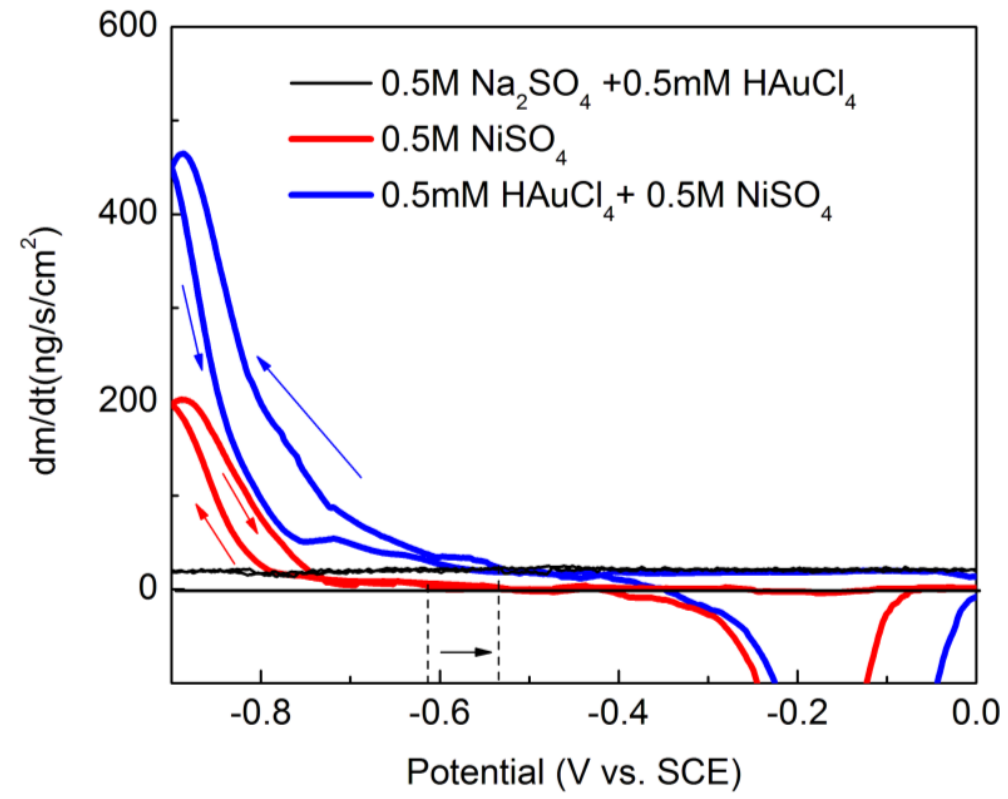

b)

Figure 5.5 Comparison of a) mass change data and b) dm/dt data for Au only, Ni only and Au $+\mathrm{Ni}$ solution extracted from Fig.5.1, 5.3 and 5.4.

For better comparison, the EQCM data for Au only, Ni only and $\mathrm{Au}+\mathrm{Ni}$ solutions are plotted together in Fig.5.5. As shown in Fig.5.5 a), the mass change for the $\mathrm{Au}+\mathrm{Ni}$ solution is much larger than the simple addition of $\mathrm{Au}$ only and $\mathrm{Ni}$ only solution. This phenomenon is more evidently demonstrated in the dm/dt data in Fig.5.5 b). For the most negative potential in the measurement, $-0.9 \mathrm{~V}$ for example, the deposition rate for alloy solution was 2.5 times larger than that of the sum of $\mathrm{Au}$ only and $\mathrm{Ni}$ only solution. In other words, the addition of $\mathrm{Au}$ into $\mathrm{Ni}$ solutions greatly increased the deposition rate of Ni. Surprisingly, as determined from $\mathrm{dm} / \mathrm{dt}$ data in Fig.5.5, Ni 
deposition was depolarized from $-0.62 \mathrm{~V}$ to $-0.53 \mathrm{~V}$ due to the addition of Au. This phenomenon, underpotential co-deposition (UPCD) has been discussed in Chapter 3 for $\mathrm{Au}-\mathrm{Cu}$ and Chapter 4 for $\mathrm{Fe}-\mathrm{Pt}$ systems [12], and was also reported for several other systems, as early as Cd-Te [13], and recently Cu-Pt [14] , Ni-Pt [15] and Co-Pt [16]. However, all these systems are thermodynamically characterized by negative enthalpy of mixing. In contrast, $\mathrm{Au}-\mathrm{Ni}$ at room temperature is practically immiscible with large positive heat of mixing [17]. Thermodynamically, the positive shift of $\mathrm{Ni}$ by Co-deposition with $\mathrm{Au}$ is not expected. We will discuss this aspect in detail in the thermodynamics section later.

To confirm that the abnormal UPCD phenomenon is a general phenomenon of electrodeposition of Au-Ni alloys, more electrolytes have been tested by adjusting the $\mathrm{Ni}$ concentration while keeping $\mathrm{Au}$ at $0.5 \mathrm{mM}$ in the solution. Shown in Fig.5.6 is the result for $0.05 \mathrm{M} \mathrm{NiSO}_{4}$. It is clearly shown that deposition rate for $\mathrm{Ni}$ was greatly enhanced by co-deposition with $\mathrm{Au}$; $\mathrm{Ni}$ deposition was shifted positive from $-0.665 \mathrm{~V}$ to $-0.58 \mathrm{~V}$. This confirms the unexpected UPCD behavior for Au-Ni electrodeposition.

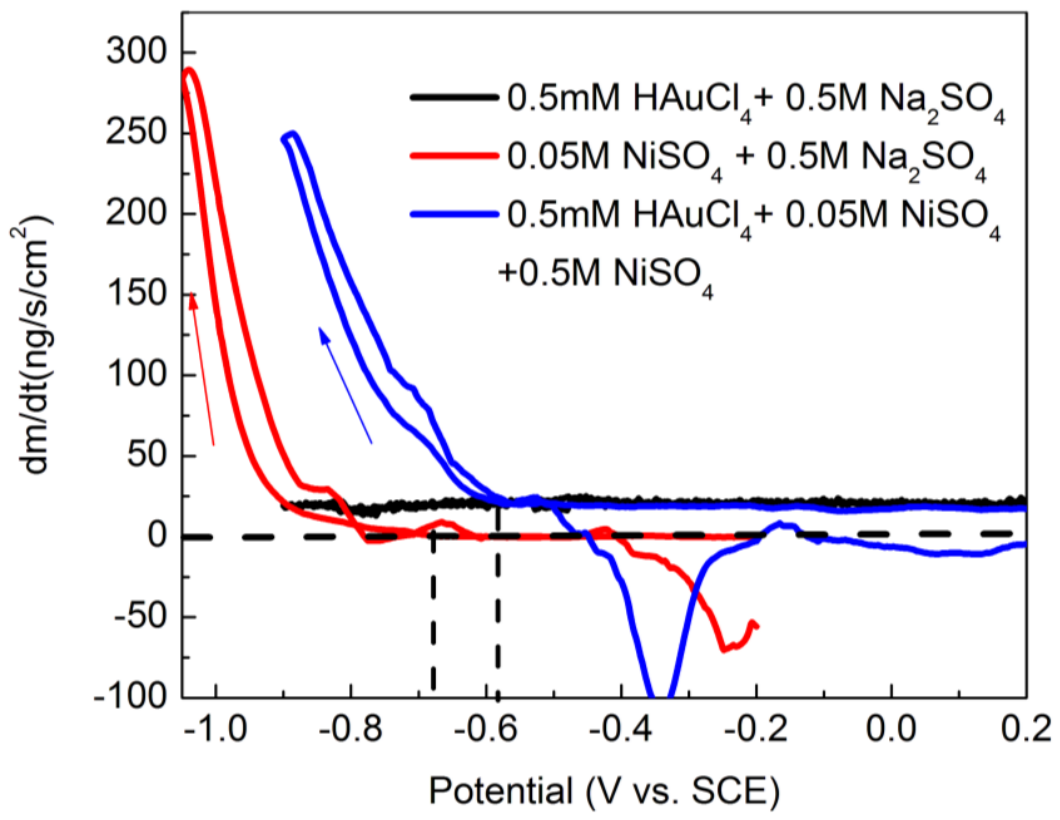

Figure 5. 6 Comparison dm/dt data for Au only, Ni only and Au + Ni solution; Ni concentration is changed from 0.5M for Fig.5.5 to 0.05M. 


\subsubsection{Composition, morphology and crystal structure of electrodeposited Au-Ni alloys}

$\mathrm{Au}-\mathrm{Ni}$ alloys were electrodeposited under constant potential from the three electrolytes shown in Table 5.1 , with $5 \mathrm{mM}, 50 \mathrm{mM}$ and $0.5 \mathrm{M} \mathrm{Ni}$ while $\mathrm{Au}$ concentration was kept constant at $0.5 \mathrm{mM}$. The composition controlled by applied potentials is shown in Fig.5.7. Recalling the EQCM data in Section 5.2, for solution with $0.5 \mathrm{M} \mathrm{Ni}, 62 \mathrm{at} . \%$ of $\mathrm{Ni}$ was co-deposited into the alloy at the potential at which $\mathrm{Ni}$ itself started depositing; for solution with $0.05 \mathrm{M} \mathrm{Ni}, 50 \mathrm{at} . \%$ of $\mathrm{Ni}$ was deposited at the onset of Ni itself. This again confirms the UPCD of Ni with Au.

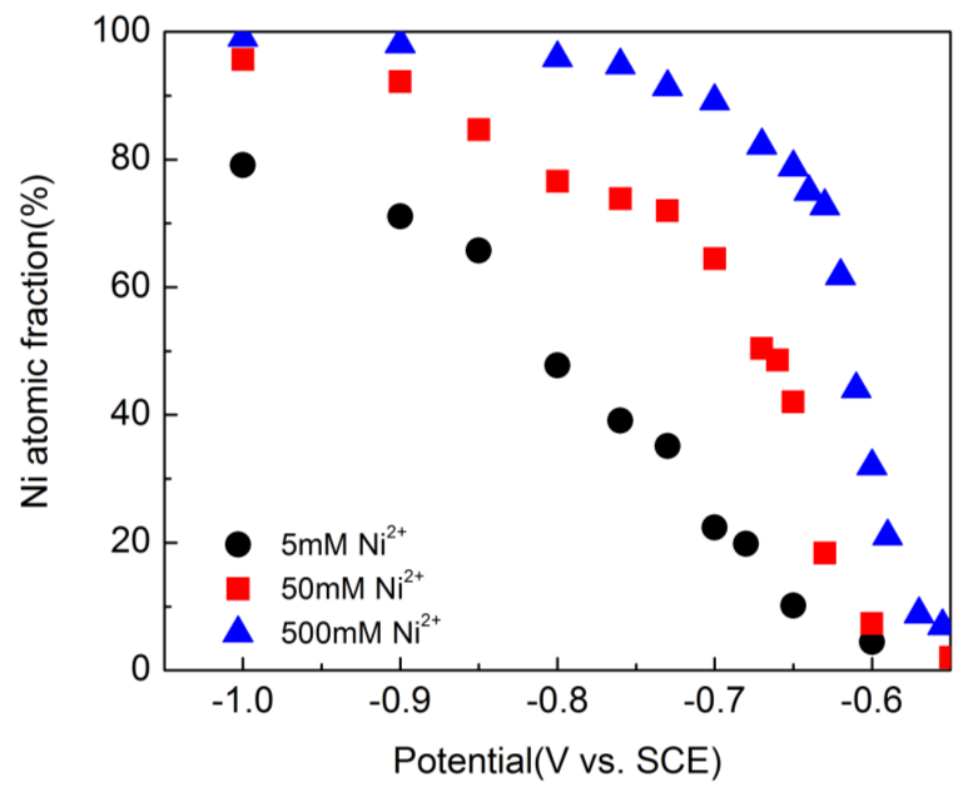

Figure 5.7 Composition vs. potential for Au-Ni alloy films ( 100nm) deposited on Ru from three electrolytes with various $\mathrm{NiSO}_{4}$ concentrations.

Morphology for Au-Ni alloys is shown in Fig.5.8. Au rich films showed rough structures of irregular shapes; with increasing Ni content, density of these structures decreased as well as their sizes. Ni rich films showed round and regular grain structures. EDS line scan for the irregular surface structures showed that they are $\mathrm{Au}$ rich compared with the overall film composition; this may be due to the lower surface energy for Au than Ni [18], leading to nanscale surface enrichment in Au. 

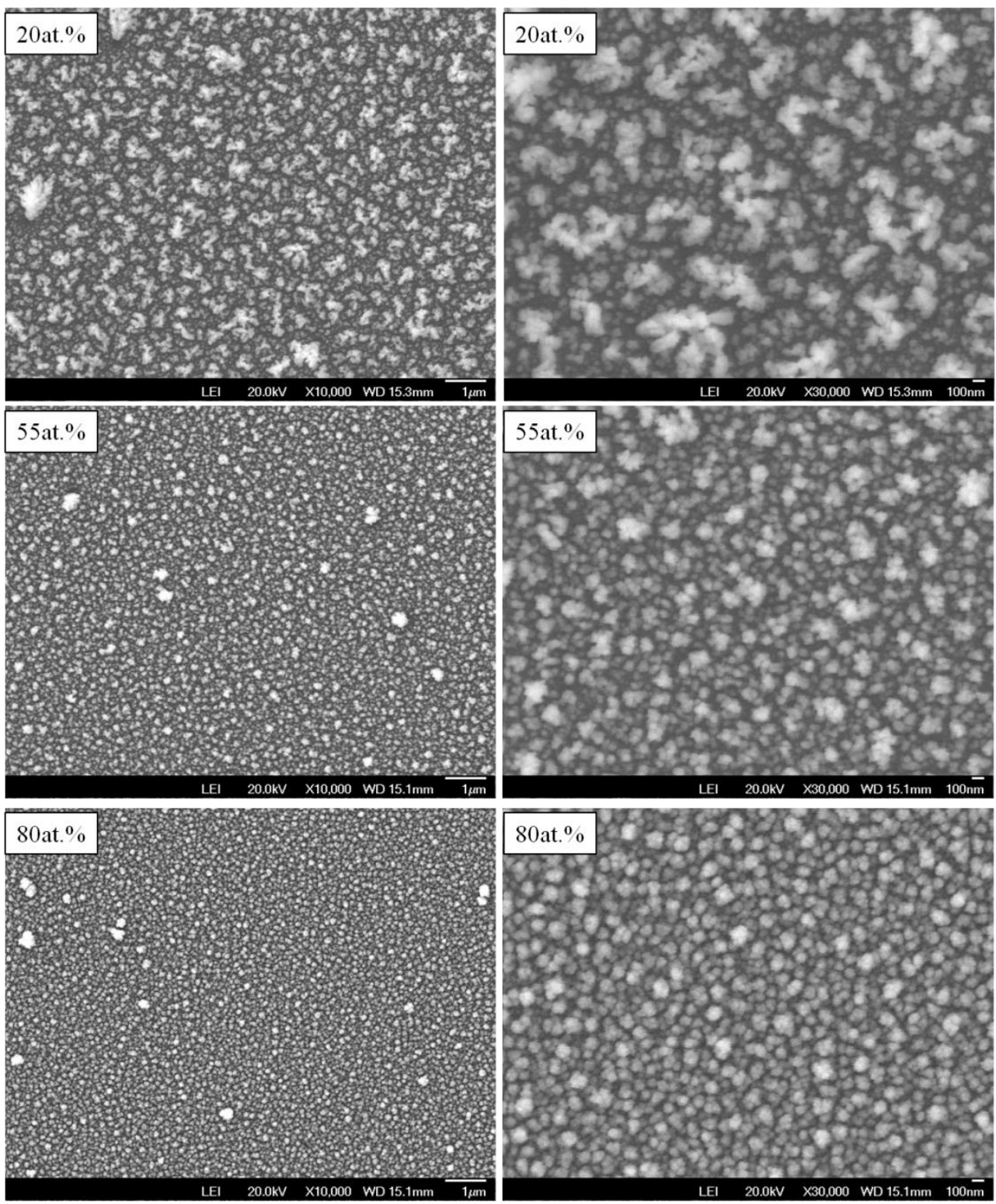

a)

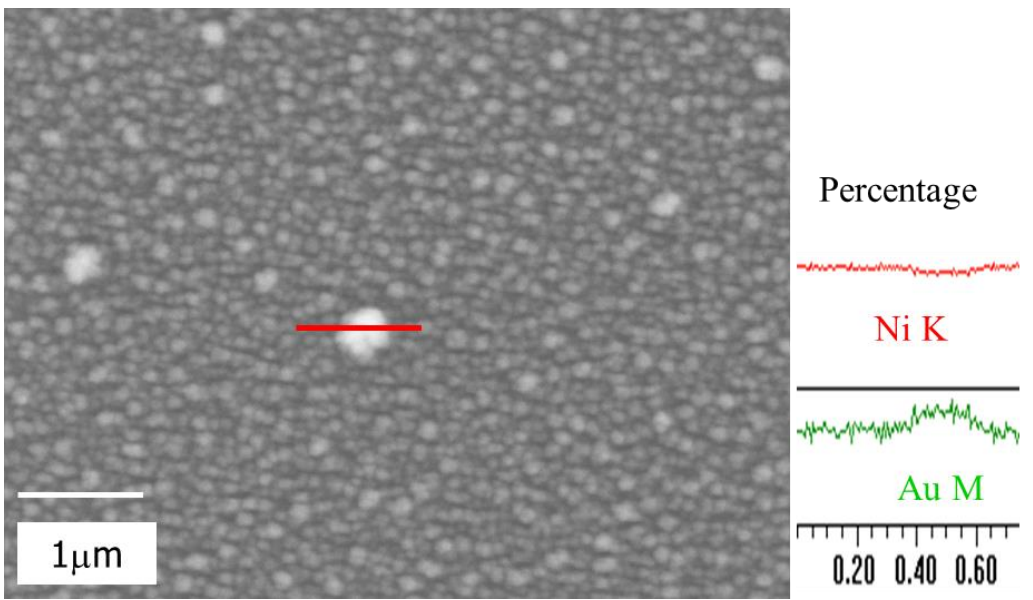

Figure 5.8 a) SEM images for Au-Ni alloys with various compositions deposited from $0.5 \mathrm{mM} \mathrm{Au}+$ $0.5 \mathrm{M} \mathrm{Ni}$ electrolyte; b) EDS line scan showing formation of Au rich structure on the surface; alloys were deposited at $-0.59 \mathrm{~V}$ for $20 \%,-0.615 \mathrm{~V}$ for $55 \%$ and $-0.66 \mathrm{~V}$ for $80 \%$. 
Crystal structure was determined using XRD. To avoid interference from the substrate peaks, several different substrates have been used. For Au rich and Ni rich films, the shift to higher angles of (111) and (200) peaks with decreasing lattice constant are consistent with the formation of solid solutions. For the intermediate composition range, the crystallinity was very poor, showing no diffraction, indicating amorphous or nanocrystalline structures.
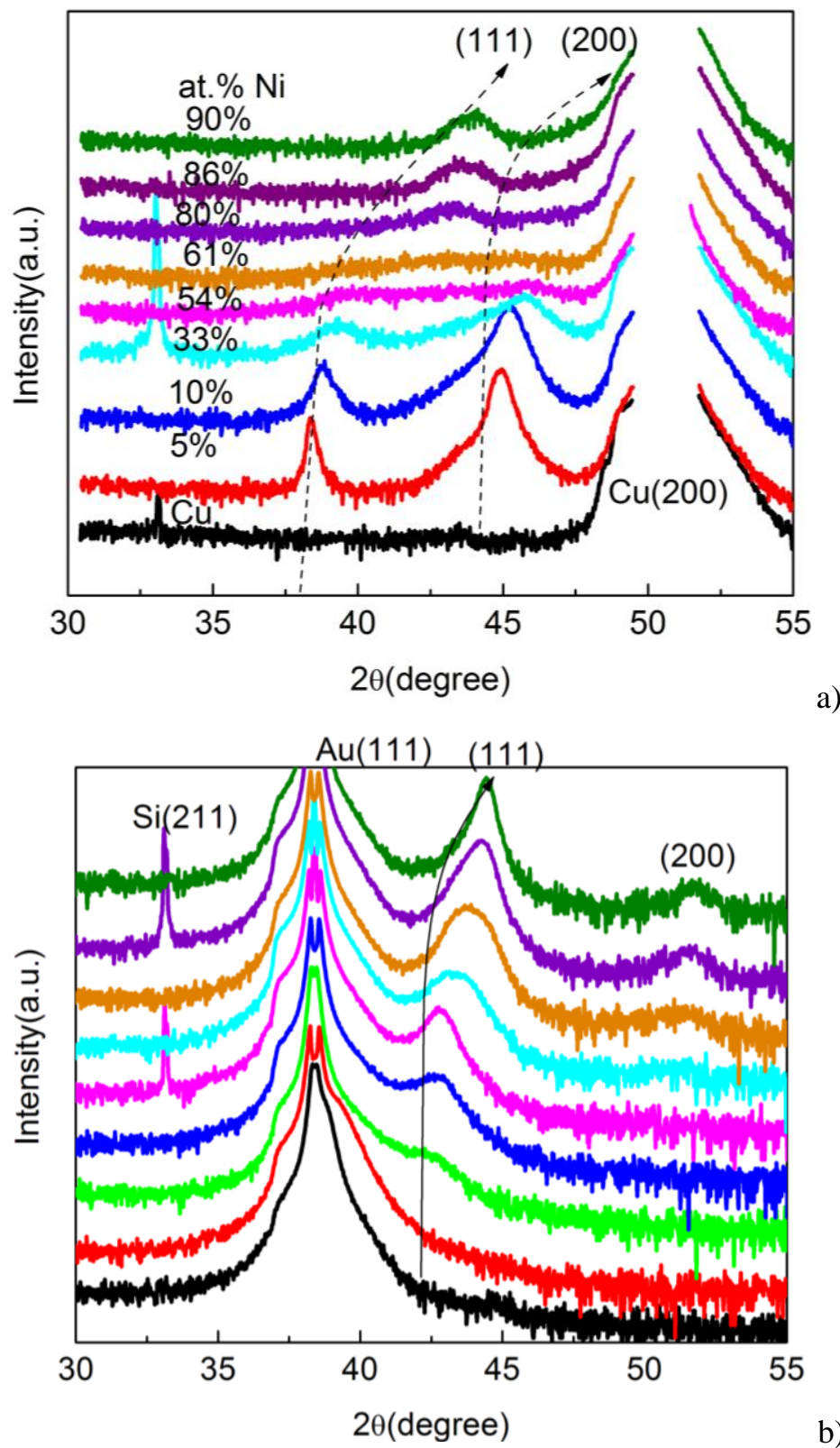

Figure 5.9 XRD patterns for $\mathrm{Au}-\mathrm{Ni}$ films from the $0.05 \mathrm{M} \mathrm{Ni}$ solutions on substrates of: a) $\mathrm{Cu} ; \mathrm{b}$ ) $\mathrm{Au}$.

The lattice constants were determined by fitting the evident (111) alloy peak and compared with the reported values for bulk alloys obtained by arc melting [19], as 
shown in Fig.5.10. Positive deviation, close to experimental bulk alloy data, is observed, lends evidence to the formation of solid solutions.

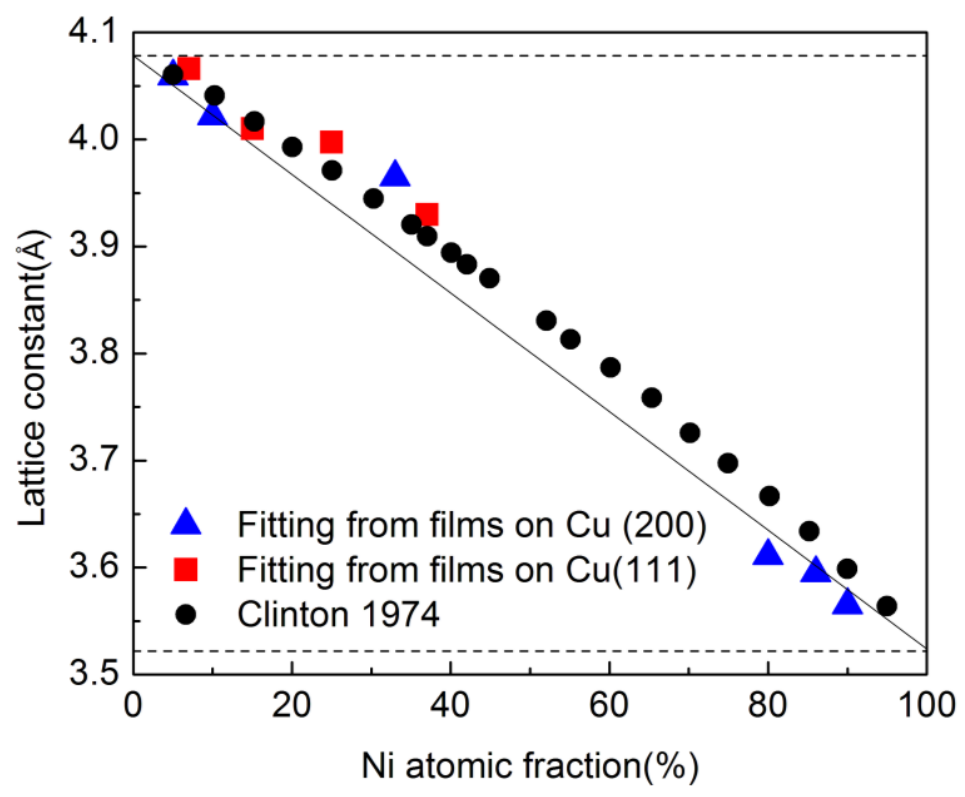

Figure 5. 10 Lattice constants by fitting alloy (111) peak from films deposited on $\mathrm{Cu}$ (111) or $\mathrm{Cu}$ (200) (patterns for films deposited on $\mathrm{Au}$ (111) were not fitted due to the difficulty in determining the exact composition); solid line is Vegard's law approximation; black solid dots are from Ref. [19].

Glancing angle XRD was also performed with the help of Dr. Zegenhagen in ESRF in Grenoble, France. As demonstrated in Fig.5.11, the alloy peaks of (111), (200) and (220) shifted to higher angles with increasing $\mathrm{Ni}$ content in the alloys, similar to the XRD data that have been shown in Fig.5.9, confirming the solid solution formation within the detection limit of XRD. The peaks of alloys were much broader comparing with either pure $\mathrm{Au}$ or $\mathrm{Ni}$, indicating that the grain sizes are reduced due to alloying of $\mathrm{Au}$ and Ni. Grain sizes were estimated by fitting the well defined diffraction peaks in Fig.5.11 and the result is shown in Fig.5.12. Grain sizes of 3-5 nm for alloys are obtained, while it is $\sim 50 \mathrm{~nm}$ for pure $\mathrm{Au}$ and $30 \mathrm{~nm}$ for pure Ni (not shown here for better comparison of alloys). 


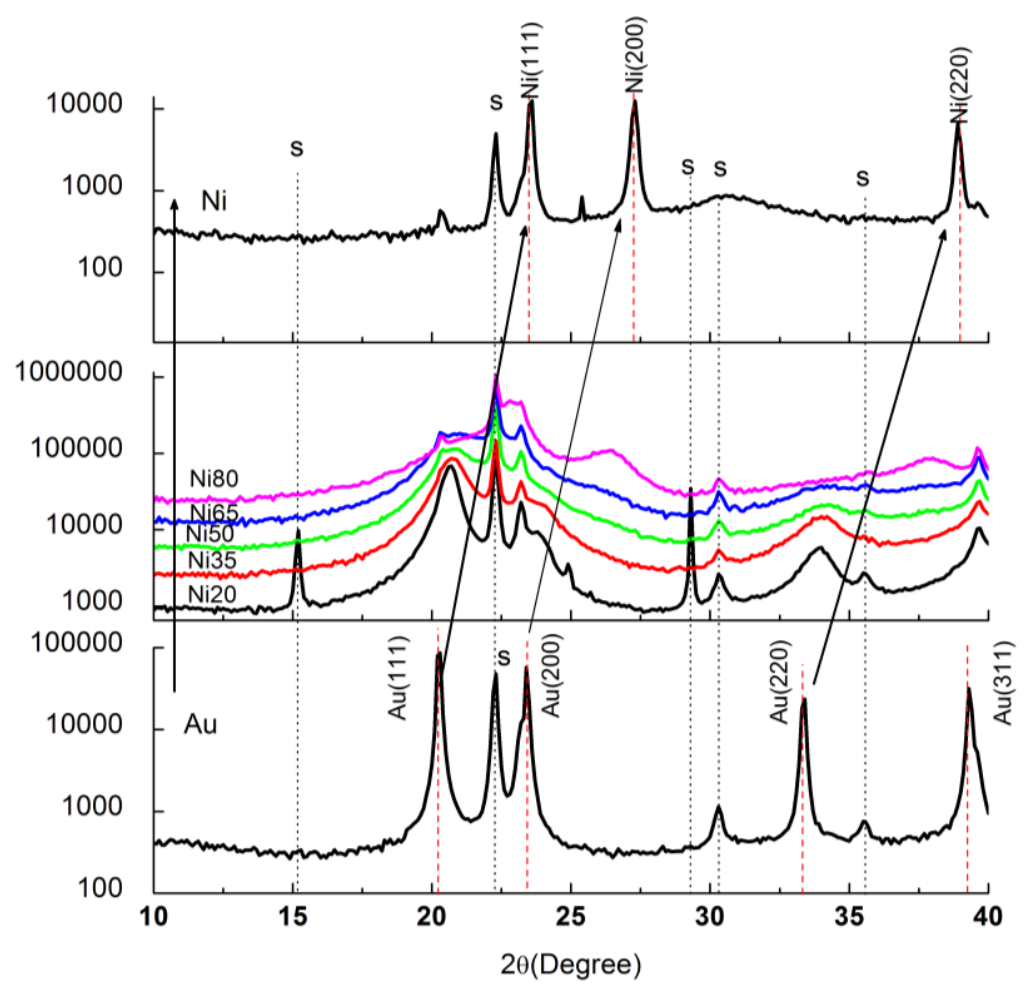

Figure 5.11 GAXRD of alloy films $(\sim 100 \mathrm{~nm})$ from the solution with $0.5 \mathrm{M} \mathrm{Ni}^{2+}$.

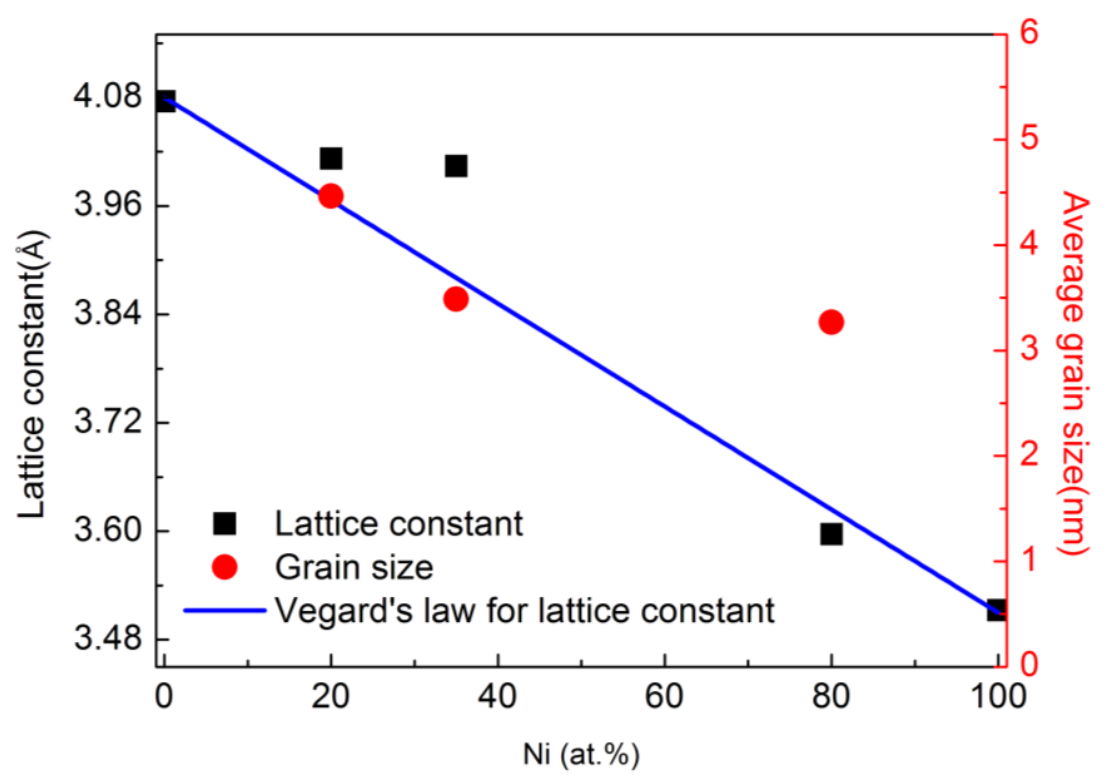

Figure 5.12 Lattice constants and grain size estimated from GAXRD in Fig.5.11.

\subsubsection{Chemical state and microstructures}

$\mathrm{XRD}$ is inadequate in proving homogeneity of nanostructured alloys due to its relatively large sampling area, which detects and collects information of mm size regions [20]. To this end, XPS was performed on the above films with glancing angle 
XRD data shown in Fig.5.13. In general, addition of valence electron charge will lead to decrease in binding energy; therefore, by alloying, elements will higher electron density will have positive chemical shift, and vice verse.
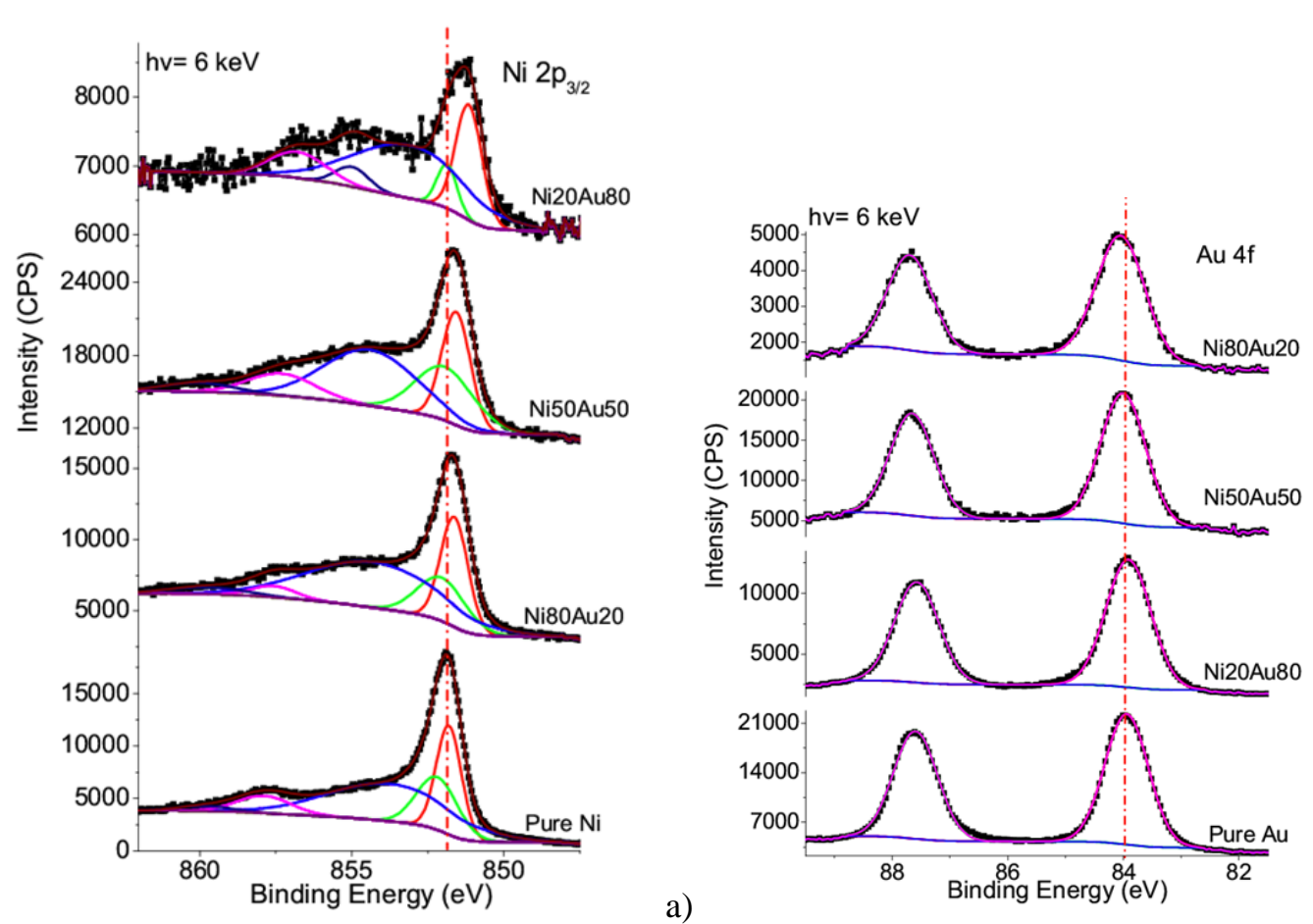

b)

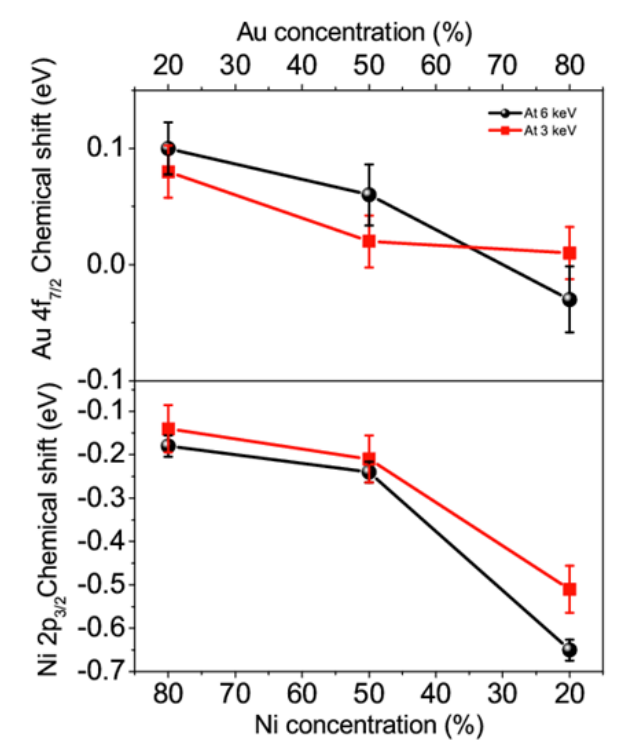

Figure 5.13 Binding energy as a function of composition for: a) $\mathrm{Ni}$; b) Au; and c) chemical shift as a function of Ni concentration.

As shown in Fig.5.13, with increasing Ni content, binding energy of Au increases, indicating decrease in valence charge for $\mathrm{Au}$; alternatively, $\mathrm{Au}$ is more likely surrounded by $\mathrm{Ni}$ than by $\mathrm{Au}$; with increasing $\mathrm{Au}$ content, binding energy of $\mathrm{Ni}$ 
decreases, indicating increase in valence charge, or $\mathrm{Ni}$ is more likely surrounded by Au. This suggests that in the Au-Ni films, Au and Ni do alloy to form solid solution structures. However, it is still unclear what the size of these structures might be. For example, do they form a homogeneous solid solution through the whole sample?

To get more details about the formed alloy structures, HRTEM experiments were performed. Figure 5.15 shows two images from $0.5 \mathrm{mM} \mathrm{Au}+0.5 \mathrm{M} \mathrm{Ni}$ solution with 40 and 60 at.\% of $\mathrm{Ni}$, respectively. Grains about $4 \mathrm{~nm}$ which are Au rich are observed; the grain sizes are quite similar to those determined from XRD in Fig.5.12. On the other hand, grain boundaries of $\sim 2-3 \mathrm{~nm}$ were rich in $\mathrm{Ni}$, and showed poor crystallinity.
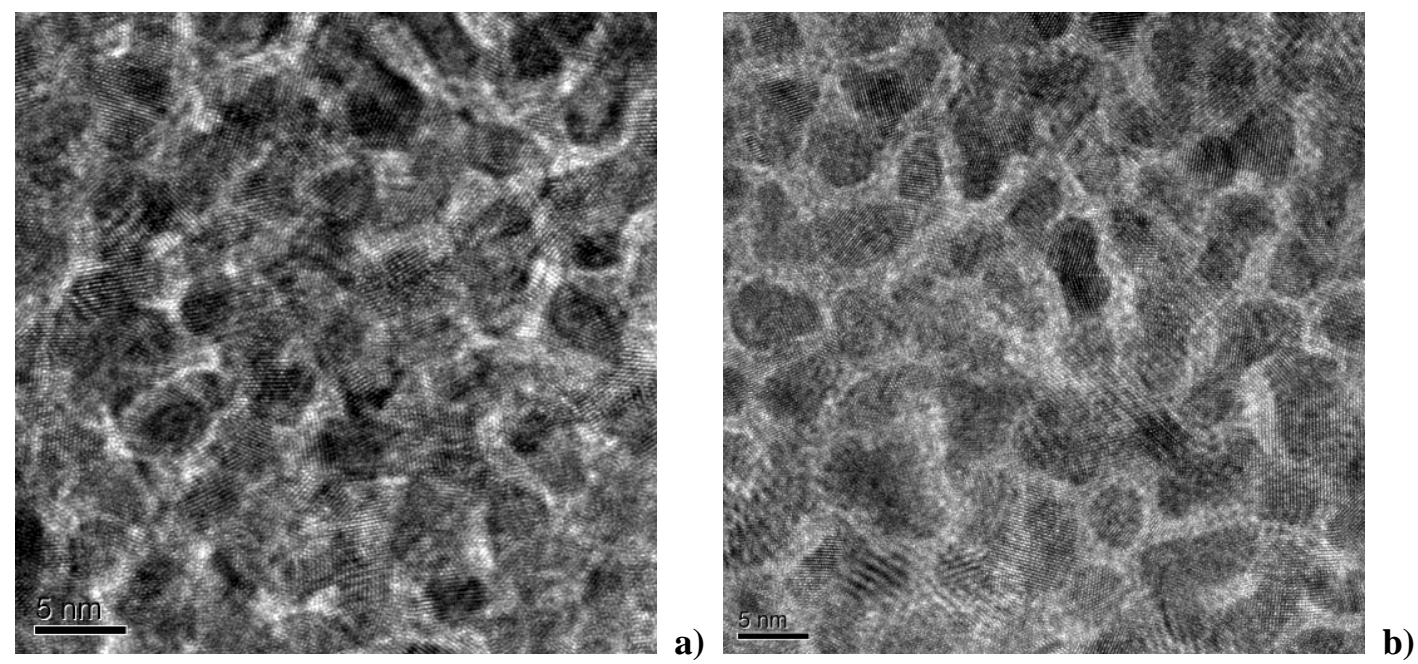

Figure 5. 15 TEM images: a) 40at.\% of Ni; b) $60 \mathrm{at} . \%$ of $\mathrm{Ni}$ deposited from $0.5 \mathrm{mM} \mathrm{Au}+0.5 \mathrm{M} \mathrm{Ni}$.

Larger grains $\sim 15 \mathrm{~nm}$ were observed for the films deposited from $5 \mathrm{mM}$ Ni solution (Fig.5.16). One possible reason is the difference in deposition rates due to the difference in Ni concentrations. According to the EQCM data shown in Section 5.2.1, assuming that $\mathrm{Au}$ deposition is under diffusion limited control for the whole alloy deposition potential range (this was confirmed for $\mathrm{Au}$ deposition from $\mathrm{Au}$ only solution by EQCM), the alloy deposition rate in monolayer (ML) was determined by summing those of $\mathrm{Au}$ and $\mathrm{Ni}$, as shown in Fig.5.17. The deposition rates for the film of $\sim 60$ at. $\% \mathrm{Ni}$ are $0.12 \mathrm{ML} / \mathrm{s}$ for $0.5 \mathrm{M} \mathrm{Ni}$ deposited at $-0.62 \mathrm{~V}$, and $0.15 \mathrm{ML} / \mathrm{s}$ for the $5 \mathrm{mM} \mathrm{Ni}$ deposited at $-0.83 \mathrm{~V}$ by taking the average of the cathodic and anodic 


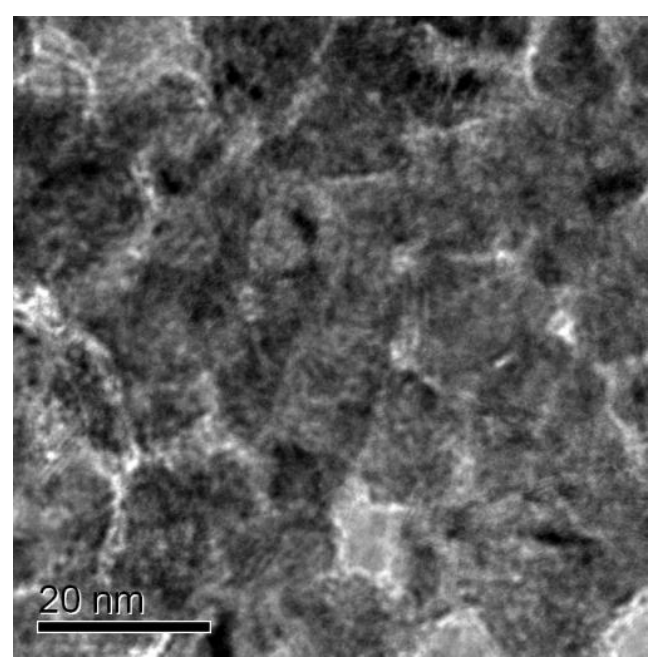

Figure 5.16 TEM image for films of 55at.\% of Ni deposited from $0.5 \mathrm{mM} \mathrm{Au}+5 \mathrm{mM} \mathrm{Ni}$.

scans. The two rates are very close to each other, ruling out the possibility of deposition rate effects on the microstructures. The result, however, is expected regarding UPCD process in the UPCD region; the overall deposition rate is controlled by the noble metal, here $\mathrm{Au}$, while the deposition rate of reactive metal is determined by the thermodynamics of the alloy system. In both cases, Au was deposited from $0.5 \mathrm{mM}$ solution with diffusion limited control, thus having the same deposition rate, and therefore the overall deposition rates are similar.

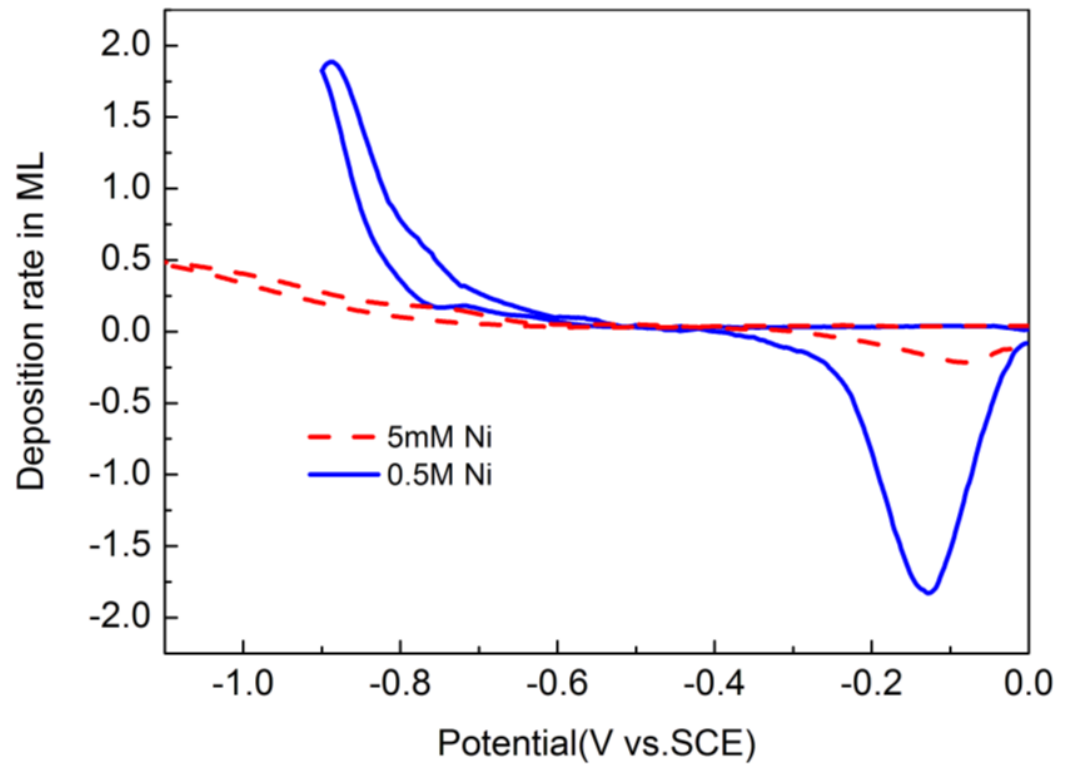

Figure 5. 17 Deposition rates in monolayer for films deposited from $5 \mathrm{mM}$ and $0.5 \mathrm{M} \mathrm{Ni}$.

The reason that different structures were obtained under similar deposition rates may be that of the surface mobility difference, which is potential dependent [21]. In the 
presence of $\mathrm{Cl}^{-}$, an enhancement of mobility on $\mathrm{Au}$ (100) surface with decreasing potential was observed [22]. For the two alloys from $5 \mathrm{mM}$ and $0.5 \mathrm{M}$ Ni here, for example, the former was deposited at $-0.82 \mathrm{~V}$ while the latter at $-0.62 \mathrm{~V}$. However, the actual behavior of surface mobility with potential is both electrolyte and substrate dependent. Indeed, these reults show that microstructures can be controlled by tuning electrodeposition parameters.

\subsection{Thermodynamics of electrodeposition of Au-Ni alloys}

The UPCD model is used to fit the experimental composition vs. potential curves to the UPCD-like region (the solid symbols), as shown in Fig.5.18. The obtained parameters are shown in Table 5.2. Futhermore, the parameters $W_{1}$ and $W_{2}$ are used to get the enthalpy of

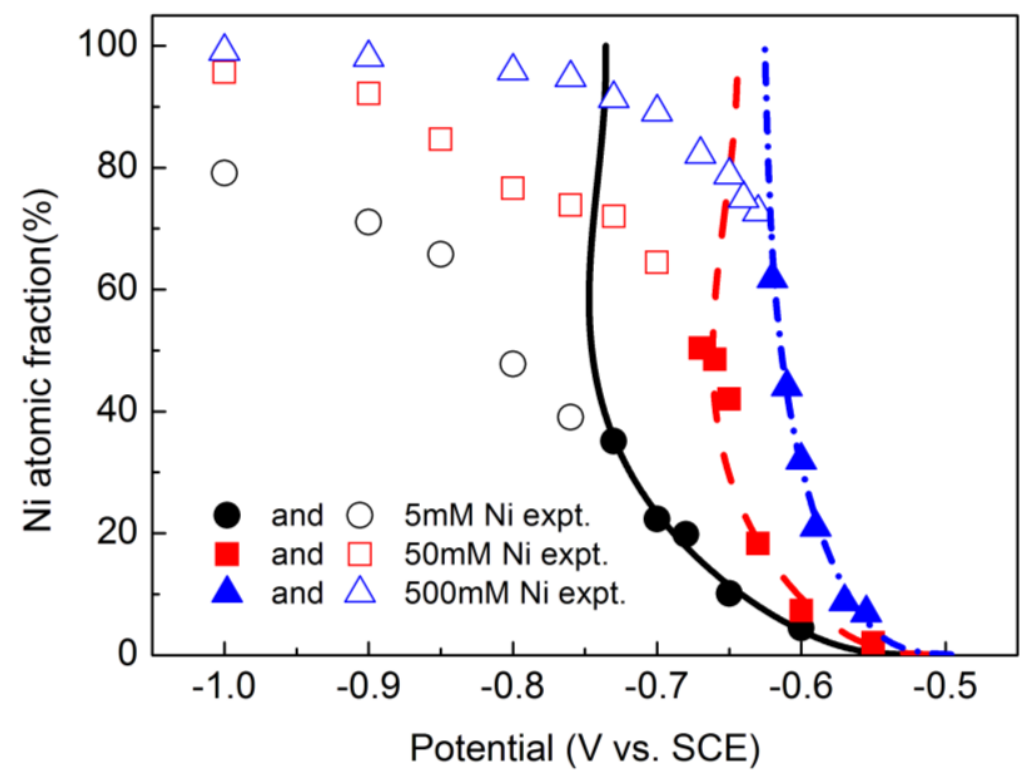

Figure 5. 18 UPCD fittings for the experimental composition vs. potential

Table 5.2 Parameters obtained by fitting composition vs. potential curve in Fig.5.18

\begin{tabular}{cccc}
\hline Ni conc. $(\mathrm{mM})$ & Equilibiurm Potential (V vs. SCE) & $\mathrm{W}_{1}(\mathrm{~kJ} / \mathrm{mol})$ & $\mathrm{W}_{2}(\mathrm{~kJ} / \mathrm{mol})$ \\
\hline 5 & -0.71 & -22.974 & 14.211 \\
50 & -0.65 & -8.72 & 19.861 \\
500 & -0.625 & -7.893 & -1.921
\end{tabular}


mixing, using the sub-regular solution model. The thus obtained results are shown in Fig.5.19a). The Au-Ni binary is known indeed to form surface alloys; various experiments in vacuum have reported that Au deposition on the low-index surfaces of $\mathrm{Ni}$ results in Au substitutionally replacing Ni, as observed both by STM[6] and by ion scattering experiments[23]. Intuitively, $\mathrm{Au}$ atoms stabilize $\mathrm{Ni}$ coordination at the

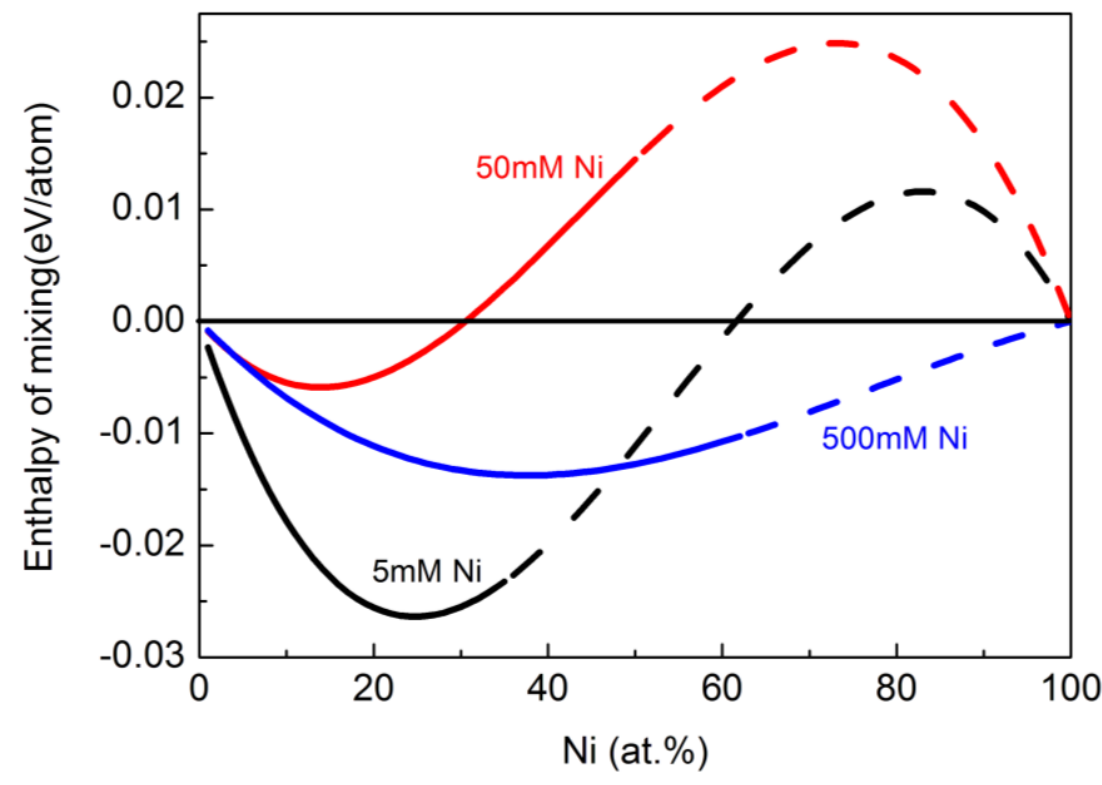

a)

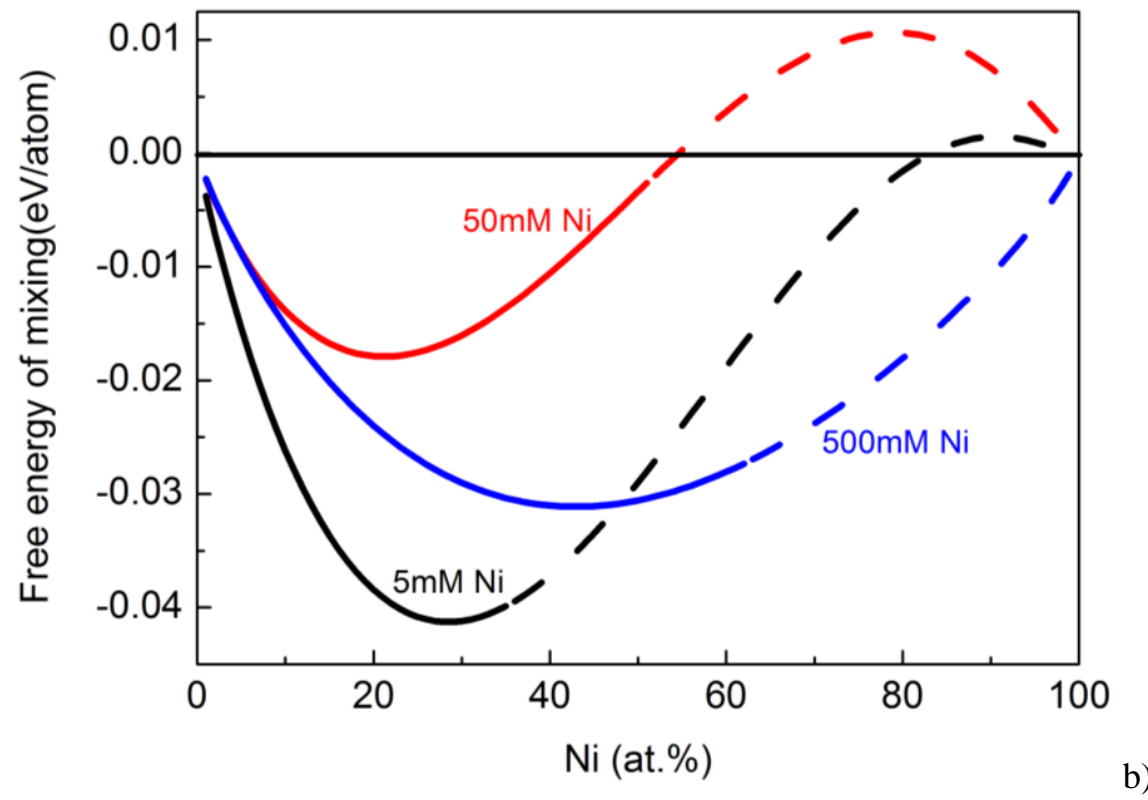

Figure 5.19 a) Enthalpy of mixing and b) free energy of mixing calculated for Au-Ni alloys grown using electrolytes with various Nickel ion concentrations. Calculations were performed using the sub-regular approximation[24] and the parametrs in Table for enthalpy of mixing and dieal solution model for entropy of mixing. 5.2. The solid parts correspond to the solid symbols in Fig.5.18.

surface by increasing electron density[6]. Due to its small value, the enthalpy of 
mixing in Fig.5.19a) is of the same order of magnitude as the entropy of mixing $\Delta S_{\text {mix }}$ for an ideal solution, and the latter cannot be neglected when considering the energetics of film formation; Fig.5.19b) shows the calculated free energy of mixing $\Delta \mathrm{G}_{\mathrm{mix}}=\Delta \mathrm{H}_{\mathrm{mix}}-\mathrm{T} \Delta \mathrm{S}_{\mathrm{mix}}$ for these films. Under quasi-equilibrium deposition conditions, $\Delta \mathrm{G}_{\text {mix }}$ should be independent of $\left[\mathrm{Ni}^{2+}\right]$, and the plots of composition vs. applied potential in Fig.5.18) should be identical, only shifted due to the variation in Ni redox potential (eqn. (1.23)); the fact that they are not suggests that the reduction kinetics of $\mathrm{Ni}$ affects in part film formation, leading to different energetics of the binary system, and therefore distinct surface atomic configurations, when deposition parameters are varied. When $\left[\mathrm{Ni}^{2+}\right]$ is 5 or $50 \mathrm{mM}$ the free energy becomes concave at high $\mathrm{Ni}$ fraction, suggesting that the Au-Ni surface alloy may become thermodynamically unstable. Solubility limits in Au-Ni surface alloys have indeed been observed; however, they are strongly dependent on the substrate being used (Ni or $\mathrm{Au}$, and their surface orientation) and on temperature, since atom exchange processes to achieve the low energy configuration may require a high activation energy[25]. Kinetic Monte Carlo calculations have also evidenced the possibility of phase separation for $\mathrm{x}_{\mathrm{Au}}>$ 0.4 and $\mathrm{T}<240 \mathrm{~K}[26,27]$. At room temperature therefore, it is likely that the electrochemically grown system is in a metastable configuration, and the enthalpy and free energy of mixing calculated herein should not be considered a truly thermodynamic property, but rather an estimate of the cohesive energy of the system.

Such cohesive energy is the sum of various contributions: the chemical term includes covalent and ionic (charge transfer) contributions to the interatomic bond between nearest neighbors; electronic effects may extend beyond the nearest neighbors, leading to metallic-type bonds; finally, atomic volume mismatch contributes an additional, repulsive term to the total energy [28]. Calculations of bonding strength in bulk Au-Ni systems using embedded atom methods[29] have shown that the chemical term is attractive (0.123 eV/atom), while the atomic volume mismatch term overcompensates the former effect, resulting in an overall repulsion. 
In electrochemical deposition, metal ions are reduced to surface adatoms by a charge transfer reaction, and film growth occurs via thermally activated transport of these adsorbed species and attachment to existing nuclei. The free energy of the growing nucleus can be decreased by minimizing the volume mismatch effect; this can be achieved in alloy growth either by forming disordered atomic arrangements - a configuration favored by a large difference in elemental atomic size [30] - or by varying the local alloy composition [31]. In this way, the atomic size mismatch that contributes significantly to the cohesive energy in the bulk should have a limited bearing during film formation, and the chemical attractive contribution may dominate film growth. STM investigations of $\mathrm{Ni}$ reduction on - and dissolution from bimetallic $\mathrm{Au} / \mathrm{Pd}$ surfaces for example have shown that the bonding strength of $\mathrm{Ni}$ to $\mathrm{Au}$ is stronger than its bond to $\mathrm{Pd}[32]$, despite the larger atomic mismatch and the unfavorable enthalpy of mixing of the alloy. This is indeed what we observe in our experiments: the UPCD process is guided by the chemical bonding interactions between $\mathrm{Au}$ and $\mathrm{Ni}$, leading to the formation of surface alloys. Thicker films are formed via the progressive deposition of surface alloys; their energetics and microstructure is governed by the interplay of atomic interactions, adatom surface mobility and the reduction rate of incoming metallic ions, the latter being partially determined by the reduction kinetics of $\mathrm{Ni}$.

\subsection{Conclusion}

$\mathrm{Au}-\mathrm{Ni}$ alloys were electrodeposited from $0.5 \mathrm{mM} \mathrm{Au}+5 \mathrm{mM} / 50 \mathrm{mM} / 500 \mathrm{mM} \mathrm{Ni}$ acidic solutions. EQCM data show depolarization of Ni co-deposition with $\mathrm{Au}$, similar to the UPCD behavior observed for $\mathrm{Au}-\mathrm{Cu}$ and Fe-Pt. Chemicall states as determined by XPS confirm solid solution formation; TEM microstructures show solid solution grains of several nms, while grain boundaries are richer in Ni. Enthalpy and free energy of mixing by fitting the experimental composition vs. potential data match that for Au-Ni surface alloys. 


\section{References}

[1] A. Dolati, M. Ghorbani, M.R. Ahmadi, J. Electroanal. Chem. 577 (2005) 1.

[2] P.C. Andricacos, C. Uzoh, J.O. Dukovic, J. Horkans, H. Deligianni, IBM J. Res. Dev. 42 (1998) 567.

[3] A.M. Molenbroek, J.K. Nørskov, B.S. Clausen, J. Phys. Chem. B 105 (2001) $5450-5458$.

[4] C. Wolverton, Comput. Mater. Sci. 8 (1997) 107-121.

[5] I.B. Ramsteiner, V.N. Bugaev, O. Shchyglo, A. Udyansky, H. Dosch, M. Asta, H. Reichert, A. Scho, 235703 (2005) 2-5.

[6] L. Pleth Nielsen, F. Besenbacher, I. Stensgaard, E. Laegsgaard, C. Engdahl, P. Stoltze, K. Jacobsen, J. Nørskov, Phys. Rev. Lett. 71 (1993) 754.

[7] P. Cavallotti, L. Nobili, A. Vicenzo, Electrochim. Acta 50 (2005) 4557.

[8] N. YAMACHIKA, Y. MUSHA, J. SASANO, K. SENDA, M. KATO, Y. OKINAKA, T. OSAKA, Electrochimi. Acta 53 (2008) 4520.

[9] E. Rouya, G.R. Stafford, U. Bertocci, J.J. Mallett, R. Schad, M.R. Begley, R.G. Kelly, M.L. Reed, G. Zangari, J. Electrochem. Soc. 157 (2010) D396.

[10] C. Dallera, L. Duò, L. Braicovich, G. Panaccione, G. Paolicelli, B. Cowie, J. Zegenhagen, Appl. Phys. Lett. 85 (2004) 4532.

[11] S. Tanuma, C.J. Powell, D.R. Penn, Surf. Interface Anal. 21 (1994) 165.

[12] D. Liang, J.J. Mallett, G. Zangari, J. Electrochem. Soc. 158 (2011) D149.

[13] D. Lincot, Thin Solid Films 487 (2005) 40.

[14] J.J. Mallett, U. Bertocci, J.E. Bonevich, T.P. Moffat, J. Electrochem. Soc. 156 (2009) D531.

[15] J.J. Mallett, E.B. Svedberg, J.E. Bonevich, A.J. Shapiro, W.F. Egelhoff, T.P. Moffat, J. Electrochem. Soc 155 (2008) D1.

[16] J.J. Mallett, E.B. Svedberg, S. Sayan, a. J. Shapiro, L. Wielunski, T.E. Madey, P.J. Chen, W.F. Egelhoff, T.P. Moffat, Electrochem. Solid-State Lett. 8 (2005) C15. 
[17] J. Wang, X. Lu, B. Sundman, X. Su, Calphad 29 (2005) 263.

[18] L. Vitos, A.V. Ruban, H.L. Skriver, J. Kollár, Surf. Sci. 411 (1998) 186.

[19] J.R. Clinton, E.H. Tyler, H.L. Luo, J. Phys. F: Met. Phys. 4 (1974) 1162.

[20] J. He, H. Sheng, J. Lin, P. Schilling, R. Tittsworth, E. Ma, Phys. Rev. Lett. 89 (2002) 12.

[21] M. Giesen, S. Baier, J. Phys. Condens. Matter. 13 (2001) 5009.

[22] N. Ikemiya, M. Nishide, S. Hara, Surf. Sci. 340 (1995) L965.

[23] D.O. Boerma, G. Dorenbos, G.H. Wheatley, T.M. Buck, Surf. Sci. 307-309 (1994) 674.

[24] H.K. Hardy, Acta Metall. 1 (1953) 202.

[25] F. Besenbacher, L. Pleth Nielsen, P.T. Sprunge, The Chemical Physics of Solid Surfaces 8 (1997) 207.

[26] W. Fan, X.. Gong, Surf. Sci. 562 (2004) 219.

[27] X. Tan, G.W. Yang, J. Alloys Compd. 467 (2009) 428.

[28] Y.A. Chang, W.A. Oates, Materials Thermodynamics, Wiley, New York, 2010.

[29] M. Asta, S. Foiles, Phys. Rev. B 53 (1996) 2389.

[30] S. Mader, J. Vac. Sci. Technol. 2 (1965) 35.

[31] J. Tersoff, Phys. Rev. Lett. 81 (1998) 3183.

[32] A. Damian, F. Maroun, P. Allongue, Phys. Rev. Lett. 102 (2009) 196101. 


\section{Chapter 6 Electrodeposition of eutectic alloys: the Ag-Cu system}

\section{Overview}

$\mathrm{Ag}-\mathrm{Cu}$ alloys with low Ag contents are potential material candidates for interconnect for microelectronics and electrical contacts [1-3], due to their improvement in tribological and anti-oxidation properties, while keeping the electrical conductivity within industrial requirements [4,5]. From the thermodynamic standpoint, as discussed in Chapter 1, Ag-Cu alloys form an eutectic system with an eutectic point of 39.9at. \% of $\mathrm{Cu}$ at $1052.1 \mathrm{~K}$ [6]. At this temperature, the solubility of $\mathrm{Cu}$ in Ag-rich alloys is 14.1at.\%, while the solubility of $\mathrm{Ag}$ in $\mathrm{FCC} \mathrm{Cu}$ is 4.9at.\% [6]. At room temperature, $\mathrm{Ag}$ and $\mathrm{Cu}$ are completely immiscible. However, it has been shown that metastable solid solutions can be obtained by various techniques, including ball mechanical milling [7] and DC sputtering [8]. As shown in Chapter 5, electrodeposition affords close control of deposition conditions far away from equilibrium, leading to the possible formation of metastable phases.

In this chapter, the effect of solution chemistry on the microstructure of electrodeposited $\mathrm{Ag}-\mathrm{Cu}$ alloys is studied. It is found by XRD methods that the $\mathrm{Ag}-\mathrm{Cu}$ system shows phase separation when deposited from acidic non complexing solution, while supersaturated solid solutions are observed in a wide compositional range when electrodeposition is performed from thiourea (TU) based complexing solution. A possible explanation of this observation based on the effect of solution chemistry and applied potential on the adatom surface mobility, and therefore on the film structure will be discussed.

\subsection{Experimental}

Deposition from non complexing solutions was investigated using a sulfate-based 
electrolyte, with variable concentrations of $\mathrm{AgNO}_{3}$ and $\mathrm{CuSO}_{4}(\mathrm{Ag}: 2-8 \mathrm{mM}, \mathrm{Cu}$ : 20-300mM) and $0.5 \mathrm{M} \mathrm{H}_{2} \mathrm{SO}_{4}$ as supporting electrolyte; a typical electrolyte contained $2 \mathrm{mM} \mathrm{AgNO}_{3}$ and $20 \mathrm{mM} \mathrm{CuSO}_{4} ; \mathrm{Cu}$ ion concentration was kept higher to facilitate the possible observation of UPCD effect.

The complexing solution contained thiourea (TU) as a complexing agent; $0.01 \mathrm{M}$ $\mathrm{Cu}_{2} \mathrm{O}$ and $2 \mathrm{mM} \mathrm{AgClO}_{4}$ were used as metal sources, $0.1 \mathrm{M} \mathrm{TU}$ was added in order to complex both metal ion species and $0.2 \mathrm{M} \mathrm{NaClO}_{4}$ was the supporting electrolyte; the pH was kept as $\mathrm{pH} 2.0$ using $\mathrm{HClO}_{4}$ and $\mathrm{NaOH}$. To make up the solution, $\mathrm{Cu}_{2} \mathrm{O}$ was first dissolved into an acidic $\mathrm{HClO}_{4}$ solution with $0.1 \mathrm{M} \mathrm{TU}$, at a temperature of $50^{\circ} \mathrm{C}$. The solution was filtered to get rid of trace amounts of undissolved impurities. Both $\mathrm{Cu}(\mathrm{I})$ and $\mathrm{Ag}(\mathrm{I})$ are complexed very strongly by $\mathrm{TU}[9,10]$. As will be shown in the next section, complexing brings the onset potential of $\mathrm{Ag}$ and $\mathrm{Cu}$ deposition closer.

Cyclic voltammetry was carried out and the corresponding mass variation due to film deposition was detected at a $\mathrm{Au}(111)$ resonator with a scan rate of $5 \mathrm{mV} / \mathrm{s}$; no electrolyte deaeration nor stirring were performed during these measurement; solutions were however deaerated for $1 \mathrm{~h}$ before every experiment by extensively purging with nitrogen gas. Substrates for thin film deposition were $100 \mathrm{~nm}$ thick, crystallographically unoriented Ru layers, which were sputter deposited onto a $5 \mathrm{~nm}$ Ta adhesion layer on top of the native oxide of $\mathrm{Si}$ (001) wafers. $\mathrm{Ag}-\mathrm{Cu}$ films were electrodeposited under constant potential control on $\mathrm{Ru}$. Film growth experiments were performed using a three-electrode cylindrical cell in the vertical configuration. The counter-electrode was a Pt mesh, and the reference was a saturated mercurous sulfate electrode (MSE). In the following all potentials will be referred to MSE ( $E_{M S E}^{0}$ $\left.=0.650 \mathrm{~V}_{\mathrm{SHE}}\right)$

Alloy composition was determined by energy dispersive spectroscopy EDS using a detector attached to a JEOL JSM-6700F scanning electron microscope (SEM). The 
ratio $\mathrm{Cu}$ to $\mathrm{Ag}$ content and possible impurities were determined using an accelerating voltage of $20 \mathrm{kV}$ and the $\mathrm{K}$ line for $\mathrm{Cu}$, the $\mathrm{L}$ line for $\mathrm{Ag}$, and the $\mathrm{K}$ line for $\mathrm{S}$. Surface morphology was characterized with the above SEM. Crystal structure was determined by X-ray diffraction, using a Panalytical X'Pert diffractomer with $\mathrm{Cu} \mathrm{K} \alpha$ radiation $(\lambda=1.5418 \AA)$ in the $\theta / 2 \theta$ geometry.

\subsection{Electrodeposition of $\mathrm{Ag}-\mathrm{Cu}$ from non complexing acidic solutions}

\subsubsection{Electrochemical studies}

Fig.6.1 shows the EQCM data for solutions with $2 \mathrm{mM} \mathrm{AgNO}_{3}$ in $0.5 \mathrm{M} \mathrm{H}_{2} \mathrm{SO}_{4}$. Mass change and dm/dt were recorded as a function of potential; Ag starts depositing at $-0.03 \mathrm{~V}$, to be compared with the value of $-0.011 \mathrm{~V}$ computed by Nernst equation. $\mathrm{Ag}$ deposition reaches a diffusion limited peak at $-0.11 \mathrm{~V}$ and is under diffusion control at potentials more negative than $-0.25 \mathrm{~V}$. In the anodic scan, Ag dissolution occurs at a potential close to the onset of reduction, indicating the high reversibility of $\mathrm{Ag}$ electrodeposition. This is due to the fast deposition kinetics of Ag, as shown by its large exchange current density $\left(\mathrm{j}_{0} \sim 1.9 \times 10^{-2} \mathrm{~A} / \mathrm{cm}^{2}, 1 \mathrm{M} \mathrm{AgClO}{ }_{4}\right.$ at $\left.25^{\circ} \mathrm{C},[11]\right)$. Here for example, the total reduction mass of $\mathrm{Ag}$ was determined to be $13644.2 \mathrm{ng} / \mathrm{cm}^{2}$, while the dissolution mass was $13643.6 \mathrm{ng} / \mathrm{cm}^{2}$; the agreement confirms the reversibity of Ag deposition. 


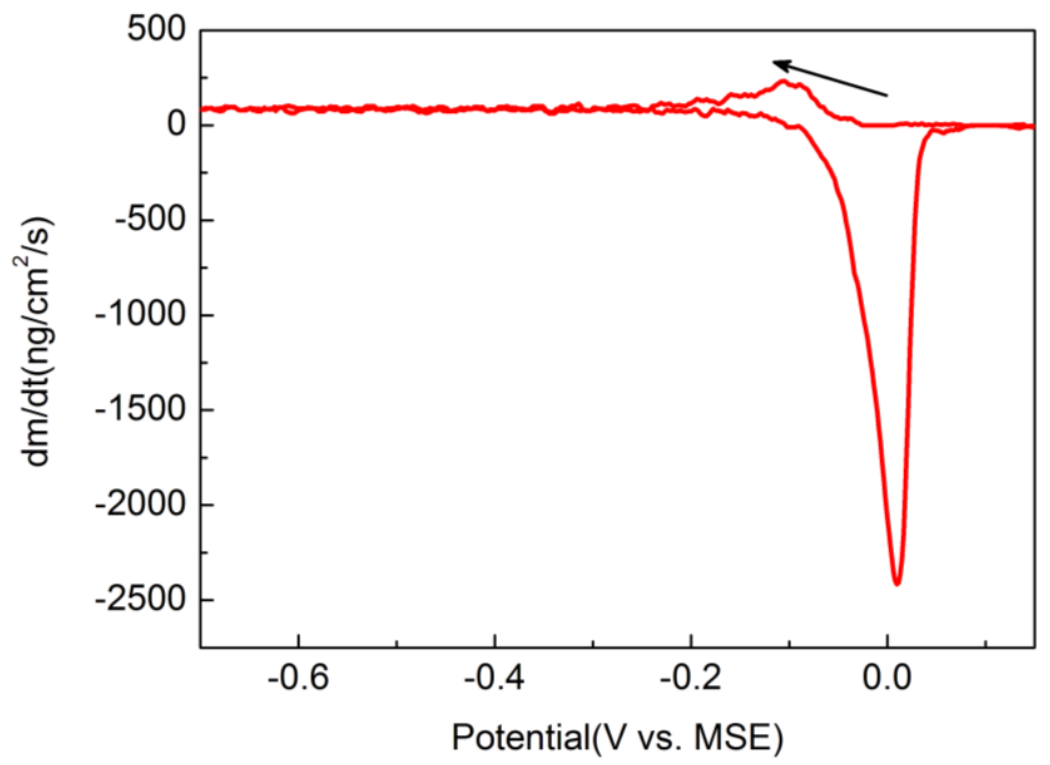

Figure $6.1 \mathrm{dm} / \mathrm{dt}$ data for $\mathrm{Ag}$ only solution as a function of potential; scan rate of $5 \mathrm{mV} / \mathrm{s}$ on $\mathrm{Au}$.

QCM data for the $\mathrm{Cu}$ only solution are displayed in Fig.6.2 and in a narrower potential range in Fig.6.3 a). $\mathrm{Cu}$ is reduced under UPD conditions on $\mathrm{Au}$ (111), starting at $-0.18 \mathrm{~V}$, and continues until $-0.32 \mathrm{~V}$, at which a coverage of $\sim-0.59 \mathrm{ML}$ is obtained (Fig.6.3b)). $\mathrm{Cu}$ bulk deposition starts at $-0.46 \mathrm{~V}$, reaches a diffusion limited peak at $-0.53 \mathrm{~V}$ and is under diffusion limited control below $-0.62 \mathrm{~V}$. On the reverse scan, $\mathrm{Cu}$ anodic dissolution starts at $-0.42 \mathrm{~V}$, slightly more positive than the onset of deposition, indicating a quite reversible kinetics of $\mathrm{Cu}$ deposition. Bulk $\mathrm{Cu}$ dissolution is completed at $\sim-0.36 \mathrm{~V}$, at which the dissolution of UPD Cu starts; the latter process continues until $\mathrm{Cu}$ is completely dissolved at $-0.16 \mathrm{~V}$. 


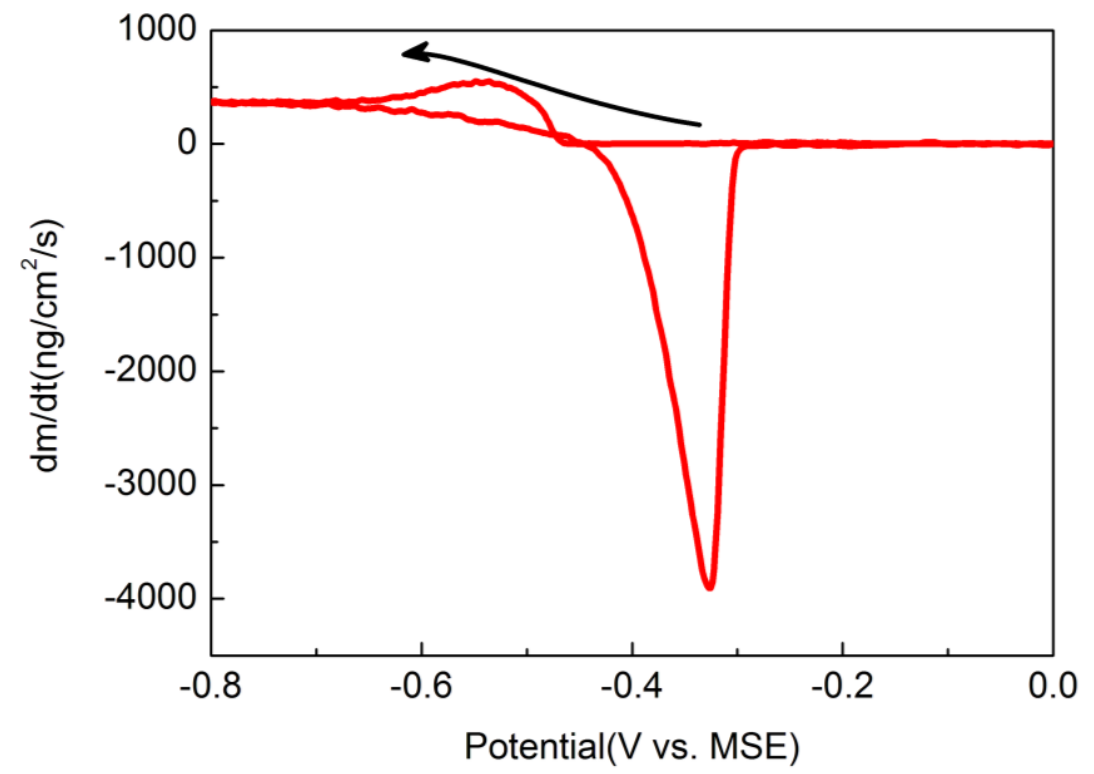

Figure $6.2 \mathrm{dm} / \mathrm{dt}$ data for $\mathrm{Cu}$ only solution as a function of potential; scan rate of $5 \mathrm{mV} / \mathrm{s}$ on $\mathrm{Au}$.
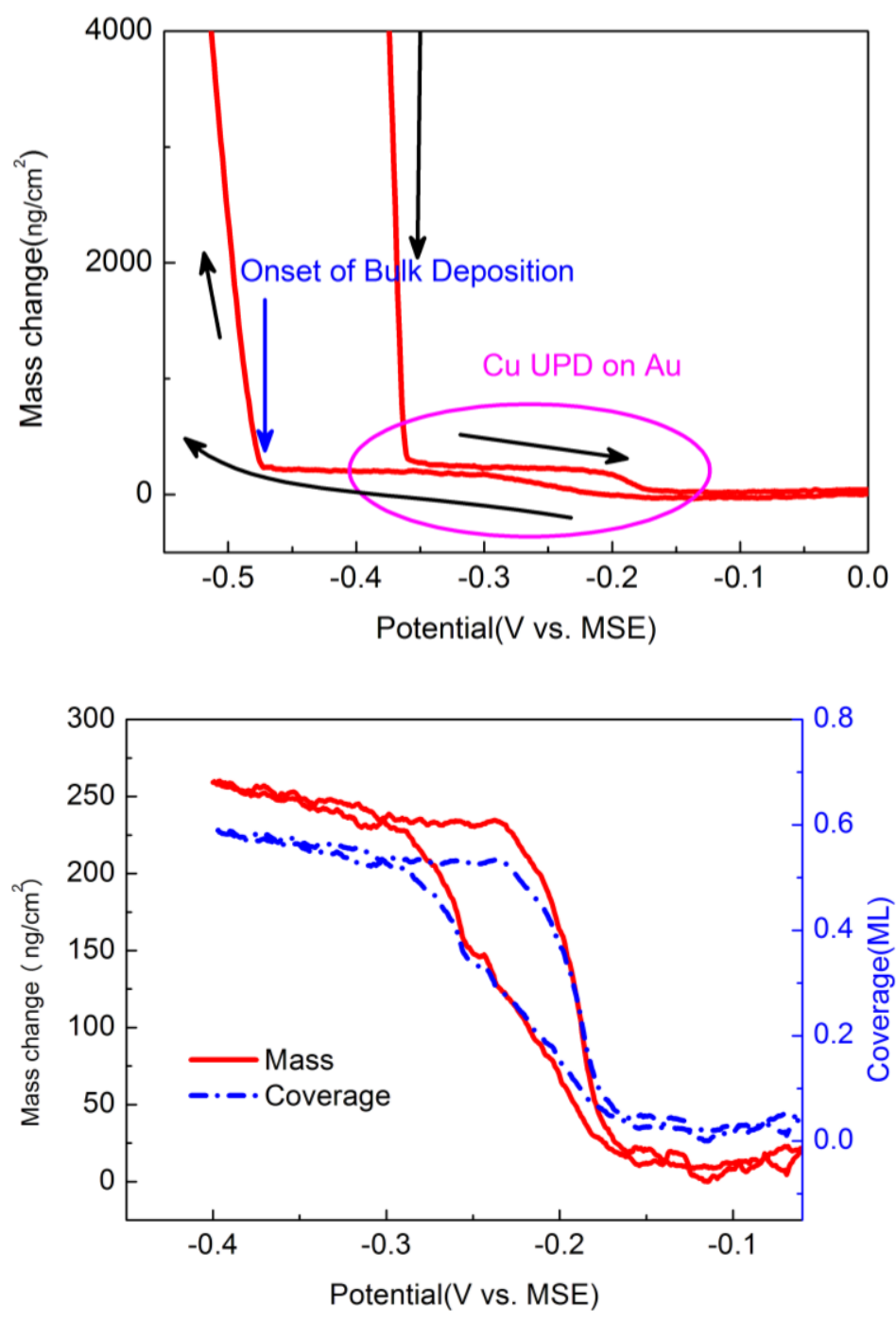

b)

Figure 6.3 a) Mass change data for $\mathrm{Cu}$ only solution showing the UPD of $\mathrm{Cu}$ on $\mathrm{Au}$; b) Coverage of the UPD layer as a function of potential. 
The QCM data for the solution with both $\mathrm{Ag}$ and $\mathrm{Cu}$ are shown in Fig.6.4. The features for onset of $\mathrm{Ag}$ deposition, $\mathrm{Ag}$ deposition peak (C1), onset of $\mathrm{Cu}$ deposition, $\mathrm{Cu}$ deposition peak (C2), $\mathrm{Cu}$ dissolution (A2) and Ag dissolution (A1) are all clearly displayed. In order to better compare the solutions (i) with only $\mathrm{Ag}$, (ii) only $\mathrm{Cu}$ and (iii) $\mathrm{Ag}$ and $\mathrm{Cu}, \mathrm{QCM}$ data are plotted together and shown in Fig.6.5. It is observed that onset of $\mathrm{Ag}$ and $\mathrm{Cu}$ deposition occurs at the same potential as in single metal solutions; in other words, no change in deposition potentials following $\mathrm{Cu}$ codeposition with $\mathrm{Ag}$ is observed. The $\mathrm{Ag}$ deposition peak in the $\mathrm{Ag}+\mathrm{Cu}$ electrolyte agrees closely with that observed in the Ag-only solution until $\mathrm{Cu}$ codeposition occurs. The rate of mass change in the $\mathrm{Ag}+\mathrm{Cu}$ solution under diffusion limitations is larger than the sum of the Ag-only and $\mathrm{Cu}$-only solutions, however. One possible

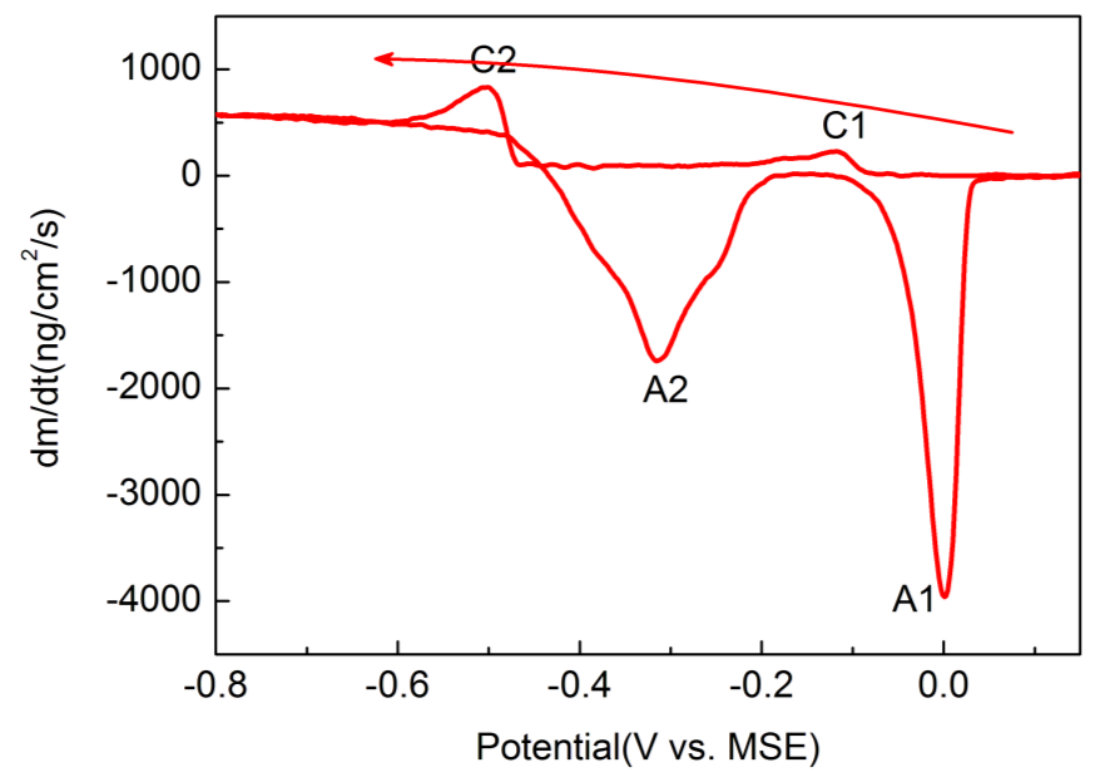

Figure $6.4 \mathrm{dm} / \mathrm{dt}$ data for the non complexedAg $+\mathrm{Cu}$ solution as a function of potential; scan rate of $5 \mathrm{mV} / \mathrm{s}$ on $\mathrm{Au}$. 


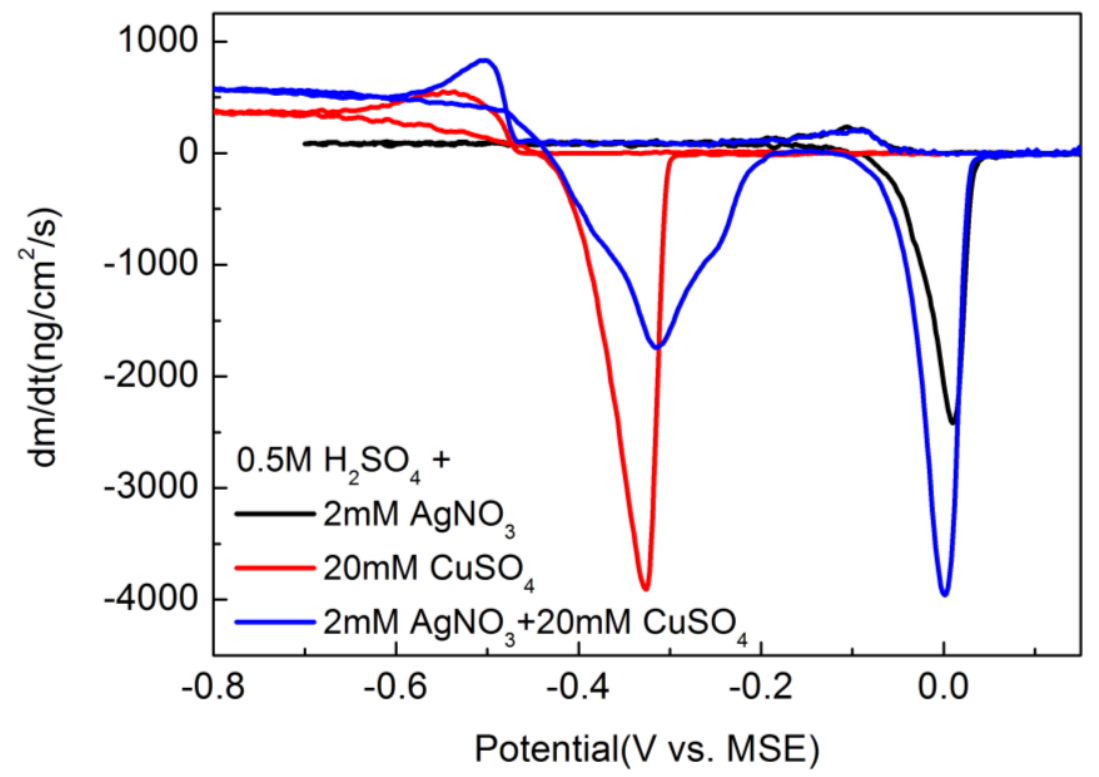

Figure 6.5 Comparison of the $\mathrm{dm} / \mathrm{dt}$ data for the solutions containing (i) $\mathrm{Ag}$ only, (ii) $\mathrm{Cu}$ only, and (iii) $\mathrm{Ag}+\mathrm{Cu}$.

reason is that before $\mathrm{Cu}$ codeposition may occur, the surface has already been roughened due to $\mathrm{Ag}$ deposition from the non complexed solution, resulting in a larger effective electrode area. This is confirmed in separate scans by stopping the scan and extracting the electrode at $-0.40 \mathrm{~V}$, which shows by visual observation a very rough surface. It is well known in fact that morphological instability of Ag deposition in the diffusion limited regime leads to a rough and dendritic surface $[12,13]$.

\subsubsection{Composition, morphology and crystal structure of Ag-Cu deposits}

$\mathrm{Ag}-\mathrm{Cu}$ films with a thickness of $200-600 \mathrm{~nm}$ were deposited at various potentials onto $\mathrm{Ru}$ substrates. Composition as a function of deposition potentials is shown in Fig.6.6 a). A sharp change from Ag-rich to $\mathrm{Cu}$-rich alloys was observed with increasing potential, very different from the gradual composition change that has been seen for $\mathrm{Au}-\mathrm{Cu}$ and $\mathrm{Fe}-\mathrm{Pt}$ alloys in chapter 3 and chapter 4, respectively. This suggests that $\mathrm{Ag}$ and $\mathrm{Cu}$ may deposit independently, possibly leading to precipitation of separated phases. To confirm this trend for composition vs. potential behavior, deposition from solutions with various ratios of $\mathrm{Ag} / \mathrm{Cu}$ was performed; results from solutions with 2 
$\mathrm{mM} \mathrm{Ag} / 0.3 \mathrm{M} \mathrm{Cu}$ and $4 \mathrm{mM} \mathrm{Ag/0.3} \mathrm{M} \mathrm{Cu}$ are shown in Fig.6.6 b). As it is clearly shown, drastic changes from less than 1 at. $\%$ of $\mathrm{Cu}$ to $\mathrm{Cu}$ rich occur within a potential interval of $\sim 20 \mathrm{mV}$, which are similar for alloys deposited from both solutions.
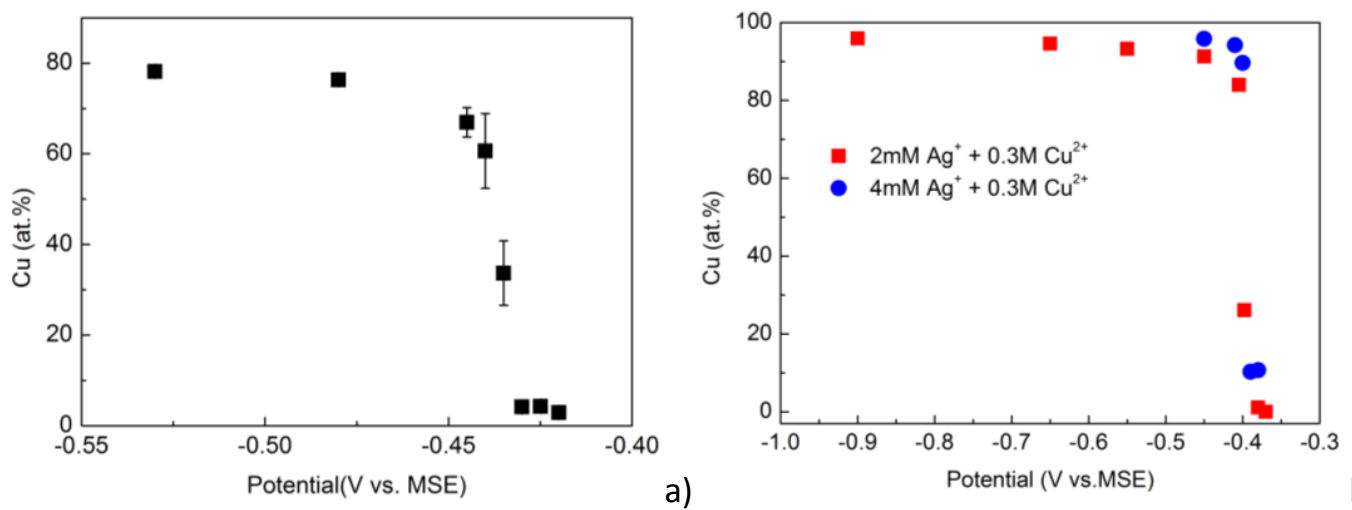

Figure 6.6. Alloy composition vs. applied potential for $\mathrm{Ag}-\mathrm{Cu}$ films deposited at various potentials: a) $2 \mathrm{mM} \mathrm{Ag}+0.02 \mathrm{M} \mathrm{Cu}$; b) $2 \mathrm{mM} \mathrm{Ag}+0.3 \mathrm{M} \mathrm{Cu}$ and $4 \mathrm{mM} \mathrm{Ag}+0.3 \mathrm{M} \mathrm{Cu}$.

The optical images of deposits with various compositions are shown in Fig.6.7. Ag-rich films are dull dark grey and very rough, due to Ag reduction occurring under diffusion-limited conditions, leading to a morphological instability; $\mathrm{Cu}$ rich films are shiny and smooth, with a reddish $\mathrm{Cu}$ color. The latter films are adherent up to a certain thickness, after which they become loose and peel off from the substrate.

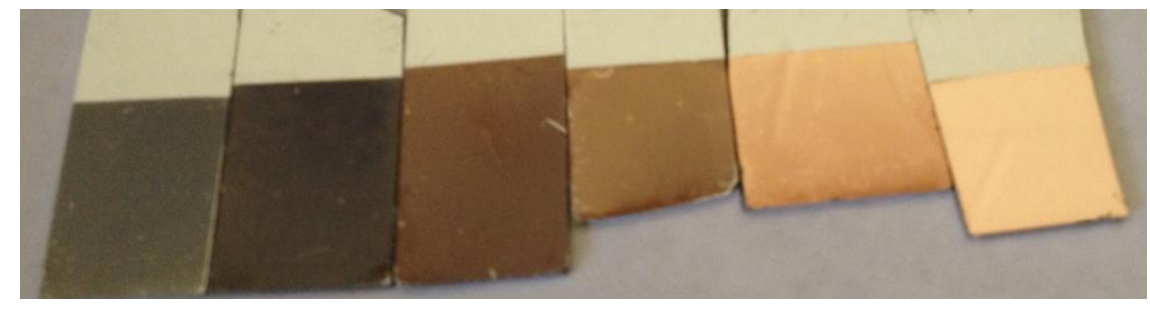

Figure 6.7. Optical images for Ag-Cu films: $\mathrm{Cu} 2.3 \mathrm{at} . \%$ to $78.5 \%$ (left to right)

The surface morphology of these films as recorded by SEM is shown in Fig.6.8. Corresponding to the optical images, Ag-rich films are rough and may form dendrites; as $\mathrm{Cu}$ content increases, dendrites disappear, while some large particle-like features form over a relatively smooth underlayer. These particles increase in density and decrease in size with increasing overpotential and thus increasing $\mathrm{Cu}$ content. In very $\mathrm{Cu}$ rich films, a smooth, uniform morphology was observed. 

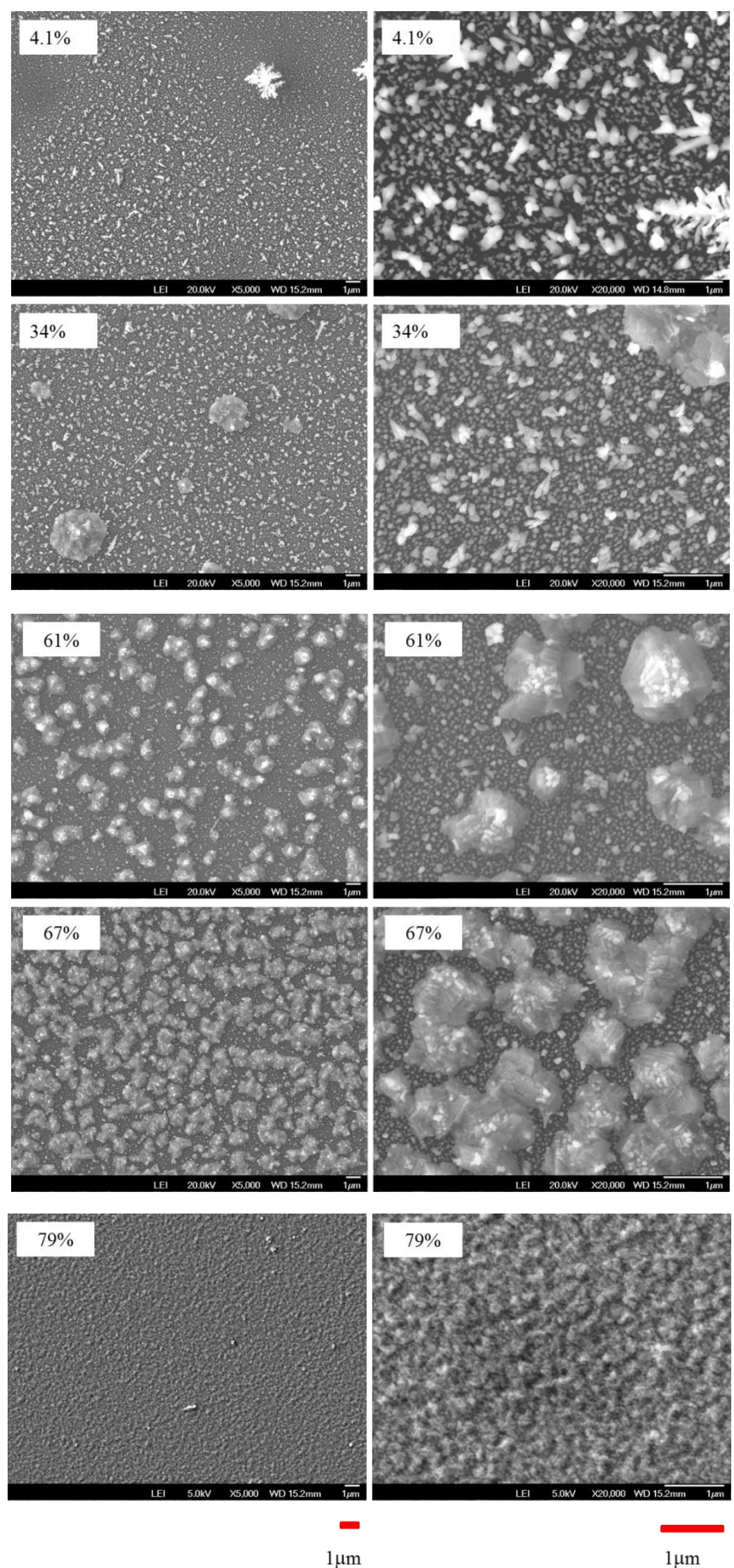

Figure 6.8. SEM surface morphology of $\mathrm{Ag}-\mathrm{Cu}$ alloys with various $\mathrm{Cu}$ contents. 

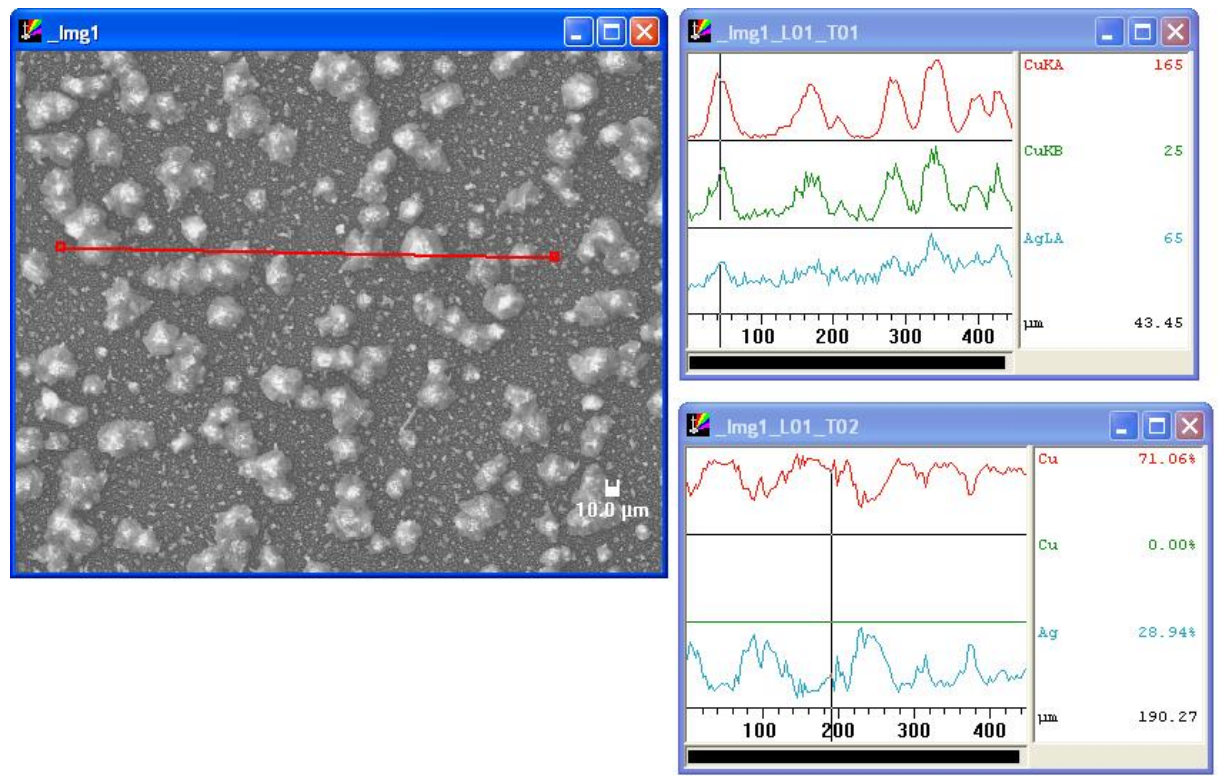

a)

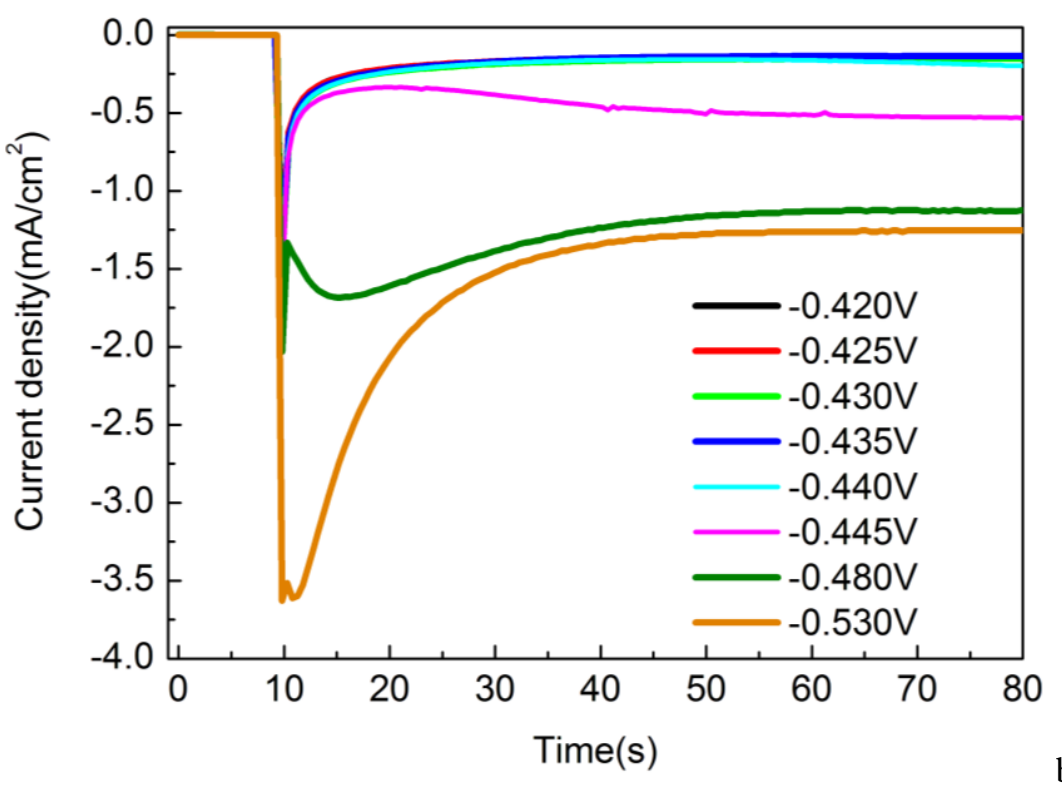

b)

Figure 6.9 a) EDS line scan for films containing 61at.\% of $\mathrm{Cu}$; b) current transients for deposition at various potential.

In order to better understand the formation of particles atop the smooth layer, EDS line scans were recorded, as shown in Fig.6.9 a). It is clearly displayed that these particles are richer in $\mathrm{Cu}$, while the initially deposited layer is Ag-rich. This behavior is consistent with an initial deposition of a Ag-rich or pure Ag film under kinetic control, followed by a depletion of $\mathrm{Ag}^{+}$concentration close to the electrode, leading to a larger $\mathrm{Cu}$ fraction being deposited. This was confirmed by the current transients recorded at various deposition potentials, as shown in Fig.6.9 b). For relatively positive potentials $(-0.42 \mathrm{~V}$ and $-0.425 \mathrm{~V})$, where $\mathrm{Ag}$ rich alloys were deposited, 
steady-state currents corresponding to the diffusion limited current of $\mathrm{Ag}$ deposition are reached immediately after double layer charging. At more negative potentials, peaks of maximum current are observed, suggesting nucleation followed by a diffusion limited growth process [14]. The higher the overpotential, the faster the nucleation, resulting in a shorter time needed to achieve full electrode coverage.
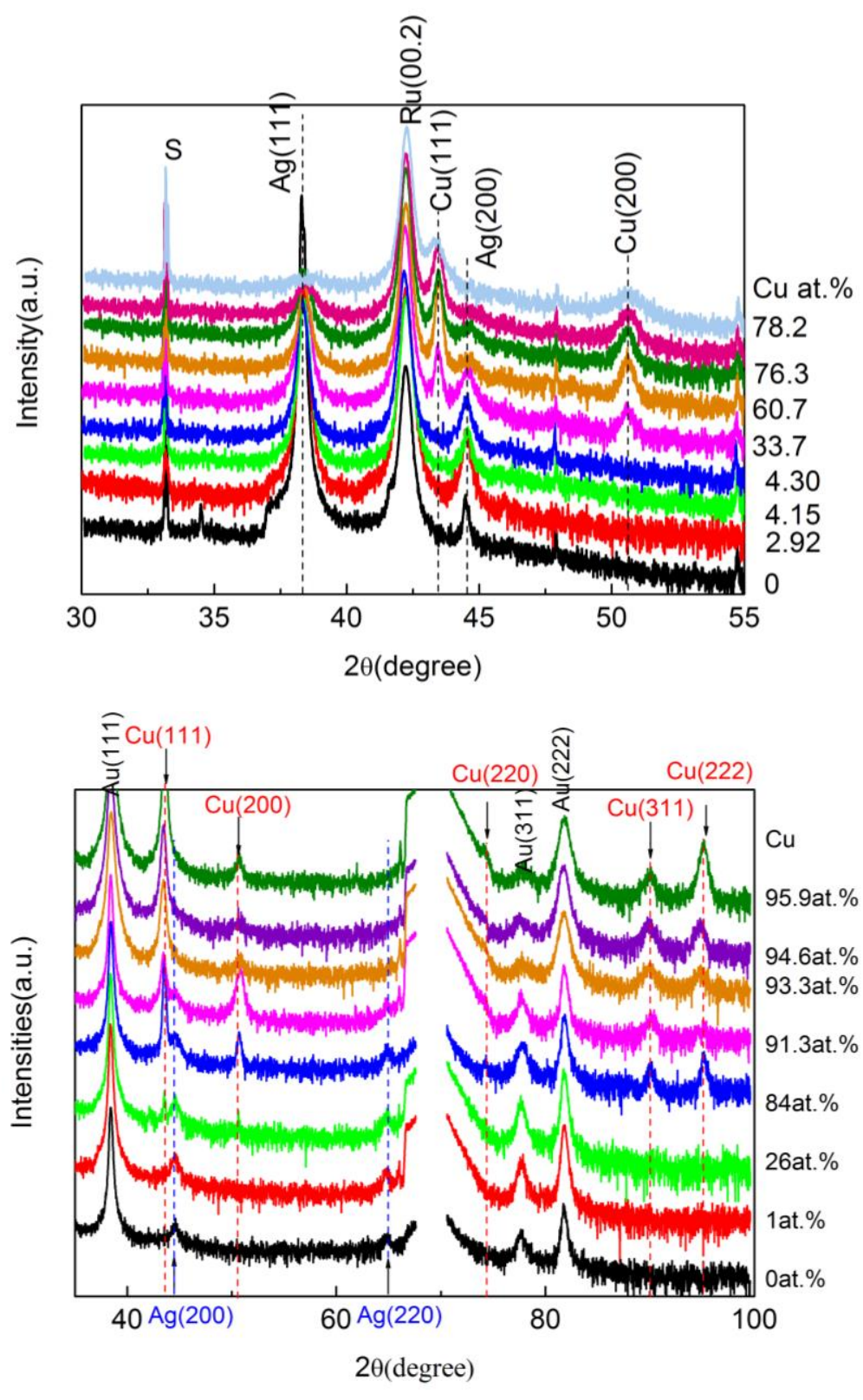

a)

b)

Figure 6.10 XRD patterns for $\mathrm{Ag}-\mathrm{Cu}$ alloys of various compositions: a) from $2 \mathrm{mM} \mathrm{Ag}+20 \mathrm{mM}$ $\mathrm{Cu}$ deposited on $\mathrm{Ru}$; b) from $4 \mathrm{mM} \mathrm{Ag}+0.3 \mathrm{M} \mathrm{Cu}$ deposited on $\mathrm{Au}$.

XRD patterns for Ag-Cu alloys are shown in Fig.6.10. For alloys containing 4.3at.\% of $\mathrm{Cu}$ or less, clear FCC (111) and (200) peaks are observed at angles corresponding 
to those of pure $\mathrm{Ag}$; for alloys with $33.7 \mathrm{at} . \%$ of $\mathrm{Cu}$ and above, evident $\mathrm{Cu}$ (111) and (200) peaks appear, together with coexisting Ag (111) and (200) reflections. Ag peaks are initially sharp, but decrease in peak height and widen as the $\mathrm{Cu}$ fraction increases, suggesting a smaller grain size. A simple way to quanfify the grain size is to fit the XRD peaks with the Scherrer equation [15]; here Lorentzian lineshapes are used to fit the $\mathrm{Ag}(111)$ and $\mathrm{Cu}(111)$ peaks representative of the precipitate $\mathrm{Ag}$ and $\mathrm{Cu}$ phases, respectively. The corresponding grain size data are shown in Fig.6.11; it is clearly seen that both the $\mathrm{Ag}$ and $\mathrm{Cu}$ phases decrease in grain size with increasing overpotential. It should be stressed that each phase might contain a small fraction of the other component, even though no peak shift was observed; the precision of the XRD data is however insufficient to verify this hypothesis and it is assumed in the following that each precipitated phase is a pure metal.

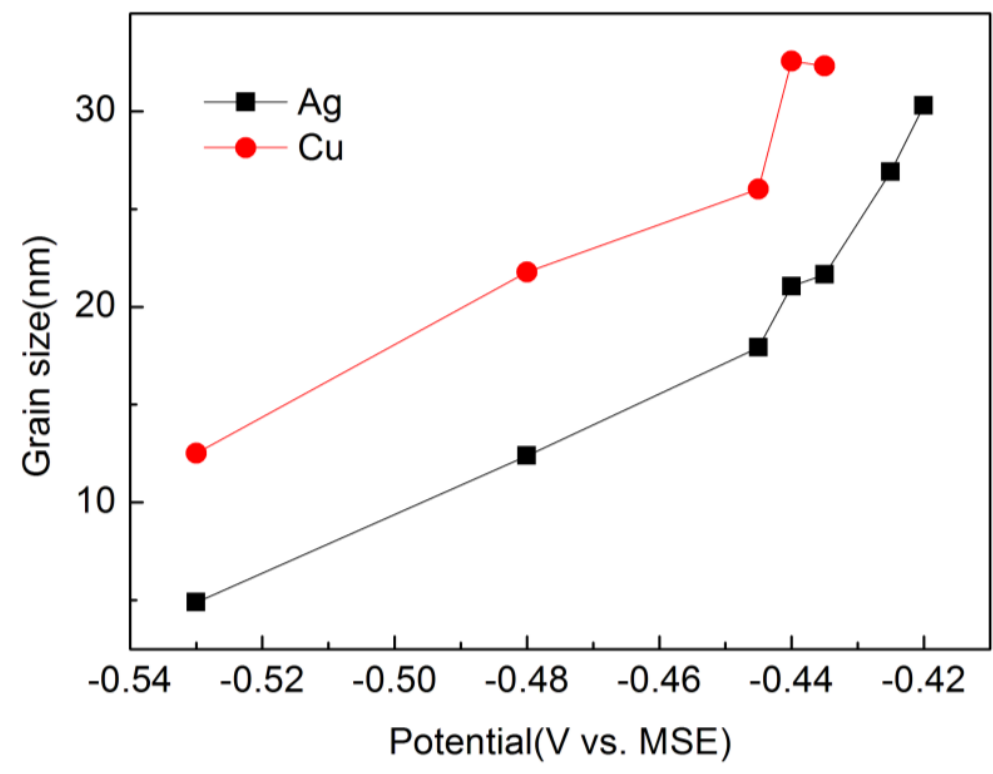

Figure 6.11 Grain size of the $\mathrm{Ag}$ and $\mathrm{Cu}$ precipitated phases, obtained by fitting the $\mathrm{Ag}(111)$ and $\mathrm{Cu}$ (111) peaks in Fig.6.10 a), using a Lorentzian line shape.

\subsubsection{Resistivity of Ag-Cu alloys}

The resistivity of the Ag-Cu alloy films was measured by a four point probe method; the data are summarized in Fig.6.12. Resistivity decreases from $2.6 \times 10^{-6} \Omega \cdot \mathrm{m}$ for Ag-rich films to $\sim 3.5 \times 10^{-8} \Omega \cdot \mathrm{m}$ for $\mathrm{Cu}$-rich films. An approximately linear 
dependence on composition should be expected for precipitate phases of $\mathrm{Ag}$ and $\mathrm{Cu}$, due to the variation in their volume fraction; additionally,, the resistivity of Ag-rich films should be lower than that of $\mathrm{Cu}$-rich ones. The observed trend is dominated by morphological features: Ag-rich films are rough and porous, leading to a higher effective resistivity. It should also be noticed that for $\mathrm{Cu}$ rich films, thicker films tend to peel off, contributing to uncertainties in resistivity measurements. Overall, the morphological instability hinders any application of deposits from the non complexed solution as electrical contacts.

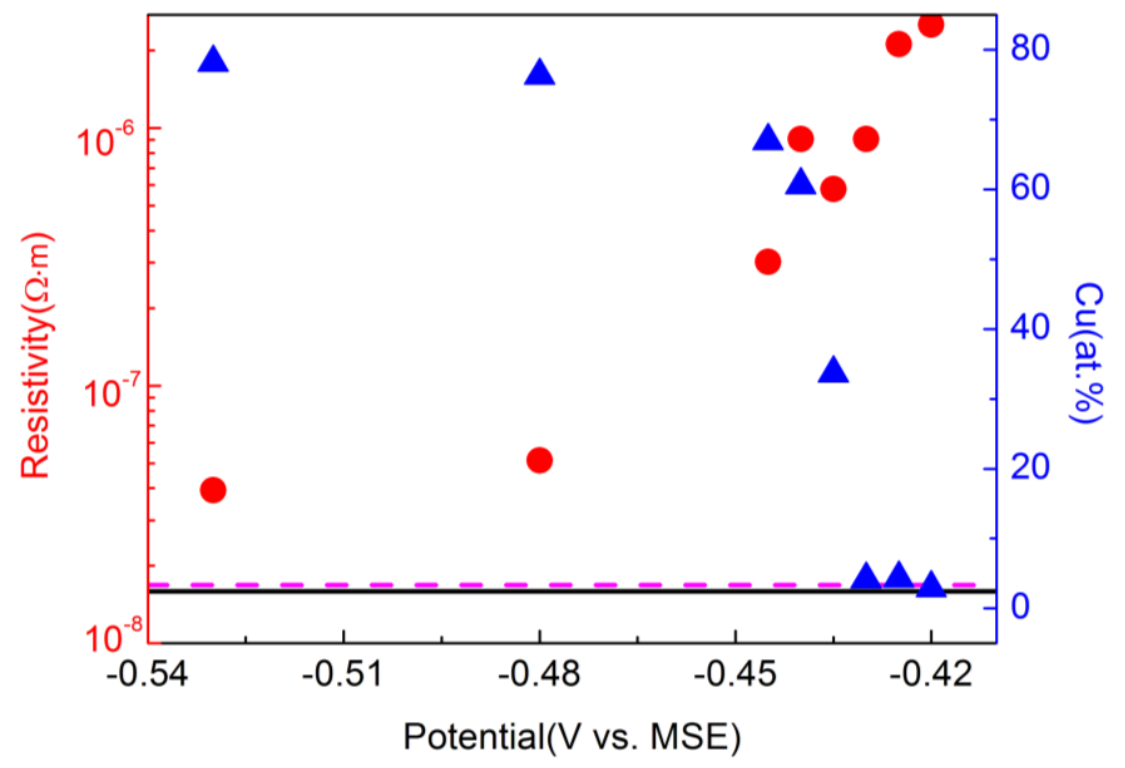

Figure 6.12 Resistivity (full circles) and composition (full triangles) of $\mathrm{Ag}-\mathrm{Cu}$ alloy films as a function of deposition potential (the black solid and magenta dash lines represent the resistivity for pure $\mathrm{Ag}$ and $\mathrm{Cu}$, respectively).

\subsection{Electrodeposition of $\mathrm{Ag}-\mathrm{Cu}$ from $\mathrm{TU}$ complexing solutions}

\subsubsection{Solution chemistry}

The complexing solution used for $\mathrm{Ag}-\mathrm{Cu}$ deposition is shown in Table 6.1. As mentioned above, TU complexes strongly both $\mathrm{Ag}^{+}$and $\mathrm{Cu}^{+}[10,16]$; the corresponding equilibria are shown in Table 6.2 for reference. The equilibria include $\mathrm{Cu}^{+}$and not $\mathrm{Cu}^{2+}$ since the latter will react with $\mathrm{TU}$ and be easily reduced to $\mathrm{Cu}^{+}$; the 
use of $\mathrm{Cu}^{+}$as $\mathrm{Cu}_{2} \mathrm{O}$ helps to minimize the unknown amount of $\mathrm{Cu}^{2+}$; we assume therefore that $\mathrm{Cu}$ ions in solution are in the cuprous form whether free or complexed with TU [17].

In order to better understand the solution chemistry in the complexing solution, we determined the concentrations of free $\mathrm{Ag}^{+}$and free $\mathrm{Cu}^{+}$by solving the system of equations shown in Table 6.2 by fixing $\mathrm{TU}$ and $\mathrm{Cu}_{2} \mathrm{O}$ and varying the concentration of $\mathrm{AgClO}_{4}$. The results are displayed in Fig.6.13; the corresponding equilibrium potentials for the $\mathrm{Ag}^{+} / \mathrm{Ag}$ and $\mathrm{Cu}^{+} / \mathrm{Cu}$ equilibria as well as their difference are calculated and shown in Fig.6.14. The gap in the deposition onset for $\mathrm{Ag}$ and $\mathrm{Cu}$ is brought down from $\sim 700 \mathrm{mV}$ (for non complexed $\mathrm{Ag}$ and $\mathrm{Cu}$ ) to less than $500 \mathrm{mV}$ in all the conditions considered; with $1 \mathrm{mM} \mathrm{AgClO}_{4}$, this gap decreases to $350 \mathrm{mV}$. In order to achieve reasonable deposition rates for alloy deposition, we set a concentration of $2 \mathrm{mM}$ for $\mathrm{AgClO}_{4}$. The small difference in the deposition onset for the two constituents may help achieve a better morphology, and further favor the formation of metastable structures, as will be shown in following sections.

Table 6.1 Solution chemistry for TU complexing solution

\begin{tabular}{cc}
\hline $\mathrm{AgClO}_{4}$ & $2 m \mathrm{M}$ \\
$\mathrm{Cu}_{2} \mathrm{O}$ & $0.01 \mathrm{M}$ \\
$\mathrm{TU}$ & $0.1 \mathrm{M}$ \\
$\mathrm{NaClO}_{4}$ & $0.2 \mathrm{M}$
\end{tabular}

pH 2.0 
Table 6.2. Equilibria and stability constants for the complexing solution

\begin{tabular}{|c|c|c|c|c|}
\hline $\begin{array}{l}\text { Complexing } \\
\text { agent }\end{array}$ & \multicolumn{2}{|c|}{ Equilibrium equations } & $\begin{array}{l}\text { Equilibrium } \\
\text { constants(Log) }\end{array}$ & $\begin{array}{l}\text { Equation } \\
\text { No. }\end{array}$ \\
\hline \multirow{6}{*}{$\begin{array}{l}\mathrm{SC}\left(\mathrm{NH}_{2}\right)_{2} \\
\text { (short for } \mathrm{L} \text { ) }\end{array}$} & \multirow{4}{*}{$\begin{array}{l}\text { Association } \\
\text { with } \mathrm{Ag}^{+}\end{array}$} & $A g^{+}+L \Leftrightarrow[A g L]^{+}$ & $K=7.11$ & $1[16]$ \\
\hline & & $A g^{+}+2 L \Leftrightarrow\left[A g L_{2}\right]^{+}$ & $\beta_{2}=10.61$ & $2[16]$ \\
\hline & & $A g^{+}+3 L \Leftrightarrow\left[A g L_{3}\right]^{+}$ & $\beta_{3}=12.74$ & $3[16]$ \\
\hline & & $A g^{+}+4 L \Leftrightarrow\left[A g L_{4}\right]^{+}$ & $\beta_{4}=13.57$ & $4[16]$ \\
\hline & \multirow{2}{*}{$\begin{array}{l}\text { Association } \\
\text { with } \mathrm{Cu}^{+}\end{array}$} & $C u^{+}+2 L \Leftrightarrow\left[C u L_{2}\right]^{+}$ & $\beta_{2}=11.1$ & $5[10]$ \\
\hline & & $2 C u^{+}+2 L \Leftrightarrow\left[C u_{2} L_{2}\right]^{2+}$ & $\beta_{22}=18.5$ & $6[10]$ \\
\hline
\end{tabular}

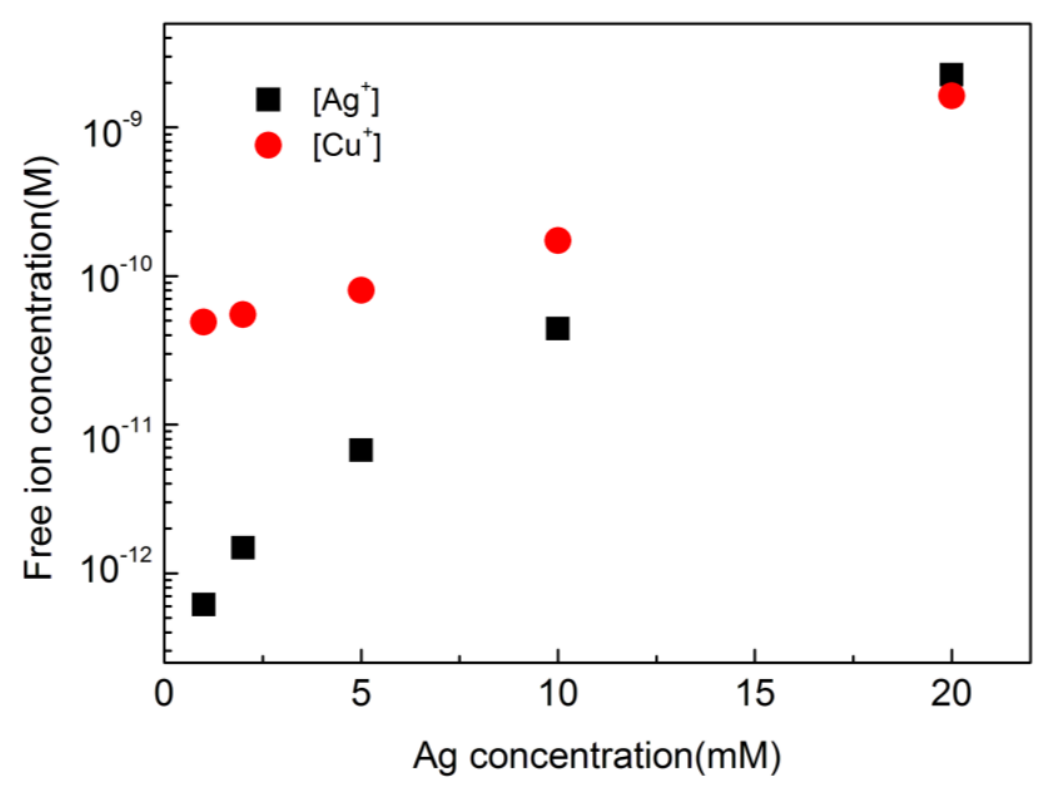

Figure 6.13 Calculated concentration of free $\mathrm{Ag}^{+}$and free $\mathrm{Cu}^{+}$by fixing the concentration of $\mathrm{Cu}_{2} \mathrm{O}$ and TU. 


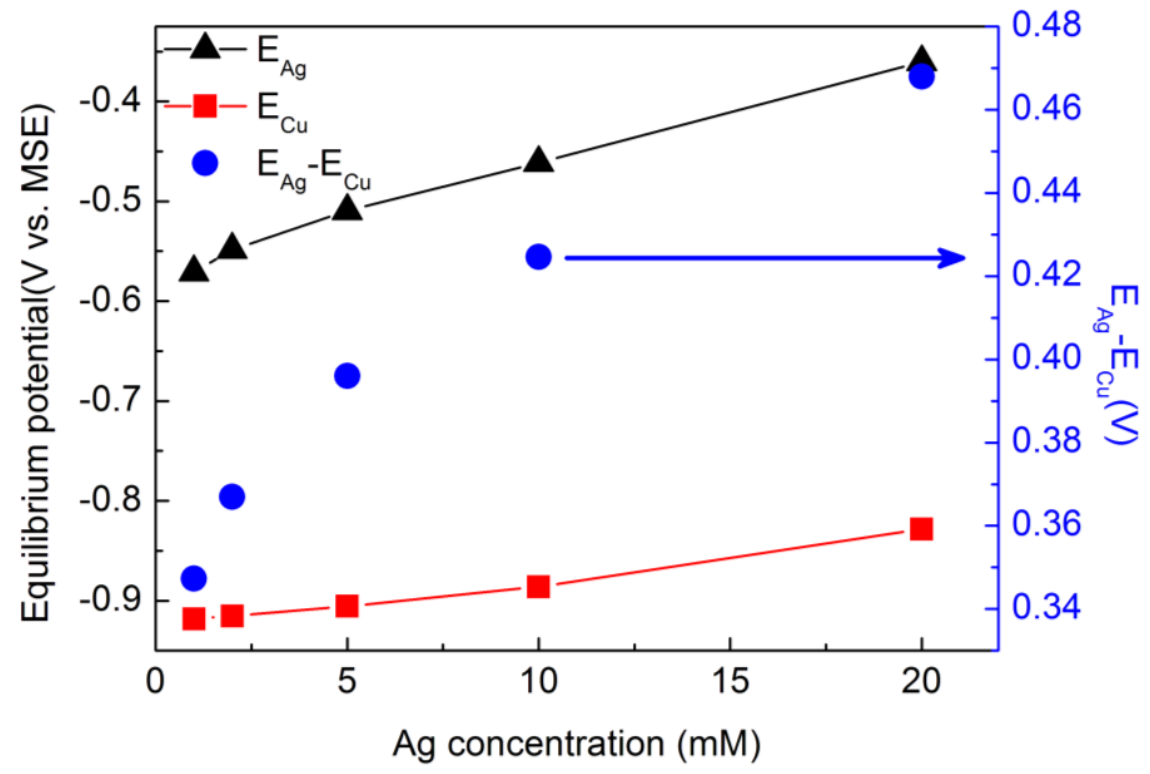

Figure 6.14 Equilibrium redox potentials for the $\mathrm{Ag}^{+} / \mathrm{Ag}$ and $\mathrm{Cu}^{+} / \mathrm{Cu}$ reactions calculated from the equilibrium concentrations shown in Fig.6.13 with $0.02 \mathrm{M} \mathrm{Cu}^{+}$and $0.1 \mathrm{M} \mathrm{TU}$ as a function of $\left[\mathrm{Ag}^{+}\right]$.

\subsubsection{Electrochemical studies}

Solution electrochemistry was studied by EQCM using CV methods. In the following, the solution without any metal ion is referred to as the background solution. Shown in Fig.6.15a) is the mass change for the background solution with the addition of $\mathrm{AgClO}_{4}$. The onset of $\mathrm{Ag}$ deposition occurs at $\sim-0.53 \mathrm{~V}$, and the diffusion limited regime is reached at $\sim-0.80 \mathrm{~V}$ as indicated by the linear change in mass over time. The various deposition regimes are shown even more clearly when the derivative of mass with respect to time is calculated and plotted together with the CV data (Fig.6.15b)). $\mathrm{CV}$ data show that bulk Ag deposition starts at $\sim-0.60 \mathrm{~V} ; \mathrm{dm} / \mathrm{dt}$ data show a similar trend. However, small increases in mass are observed already starting at $\sim 0.54 \mathrm{~V}$. This may be due to Ag UPD on Au substrate, as reported for example by Herrero [18]. The onset of Ag deposition as determined by EQCM data agrees well with that calculated from chemical equilibria and shown in Fig.6.14. The diffusion peak observed in the $\mathrm{dm} / \mathrm{dt}$ trace corresponds well with that observed in the CV; finally, a diffusion plateau is observed starting at $-0.9 \mathrm{~V}$. It should be noted that $\mathrm{Ag}$ deposition and dissolution start at potential values that are very close, suggesting that complexation changes the 
reversibility of Ag deposition only to a limited extent.
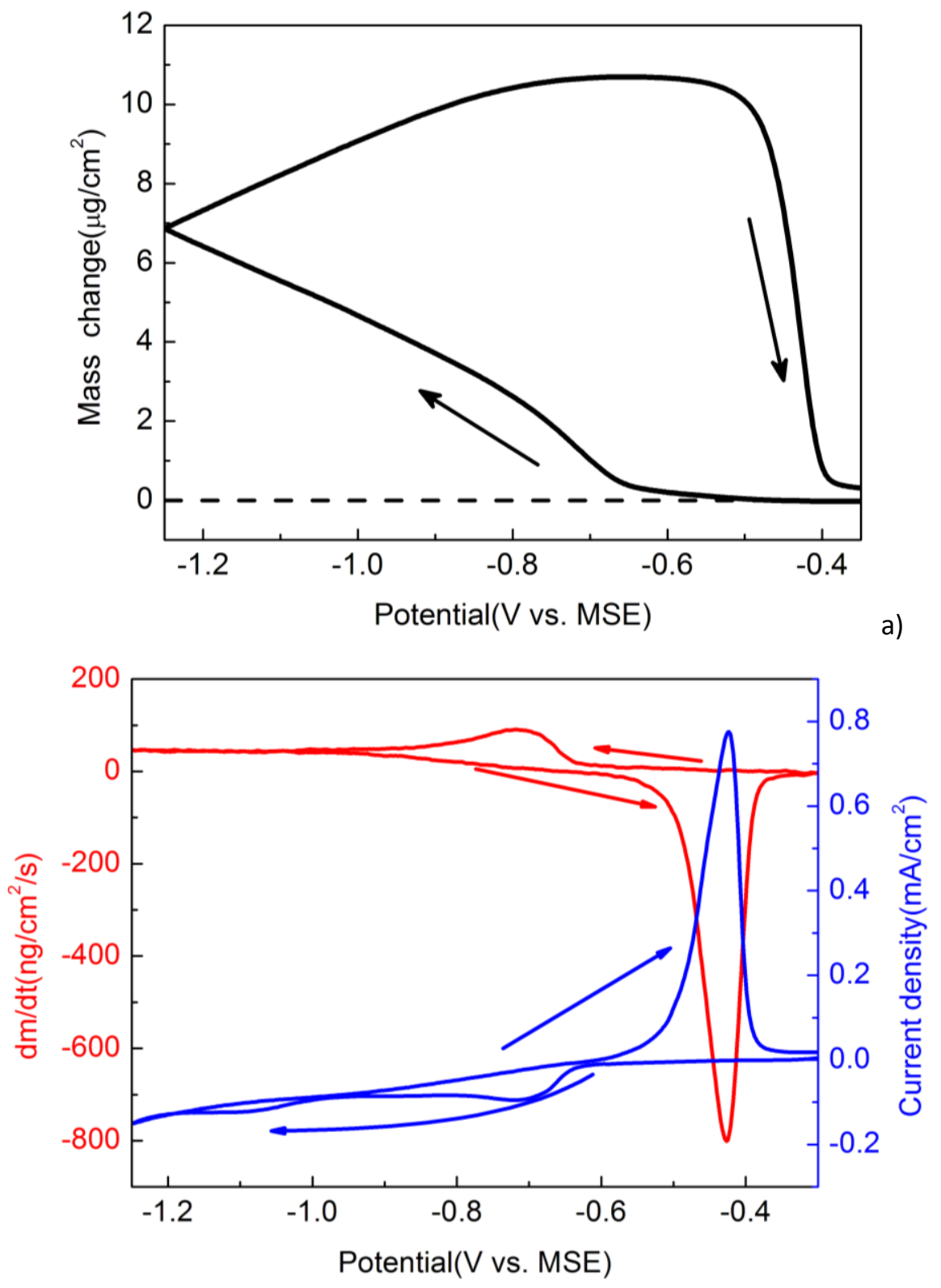

b)

Figure 6.15 a) Mass change and b) $\mathrm{CV}$ and $\mathrm{dm} / \mathrm{dt}$ data for the background solution with the $\mathrm{Ag}$ salt added.

The partial current for $\mathrm{Ag}$ can be extracted from the $\mathrm{dm} / \mathrm{dt}$ data and the cathodic current efficiency (Fig.6.16), where the latter is calculated as the ratio of the $\mathrm{Ag}$ partial current to the total current (Fig.6.15b)). Efficiency drops from $\sim 100 \%$ at the initial stage of $\mathrm{Ag}$ deposition to $\sim 29 \%$ at $-1.25 \mathrm{~V}$, due to the parasitic HER, which is calculated to start at $-0.77 \mathrm{~V}$ according to Nernst equation for a $\mathrm{pH} 2.0$ solution. 


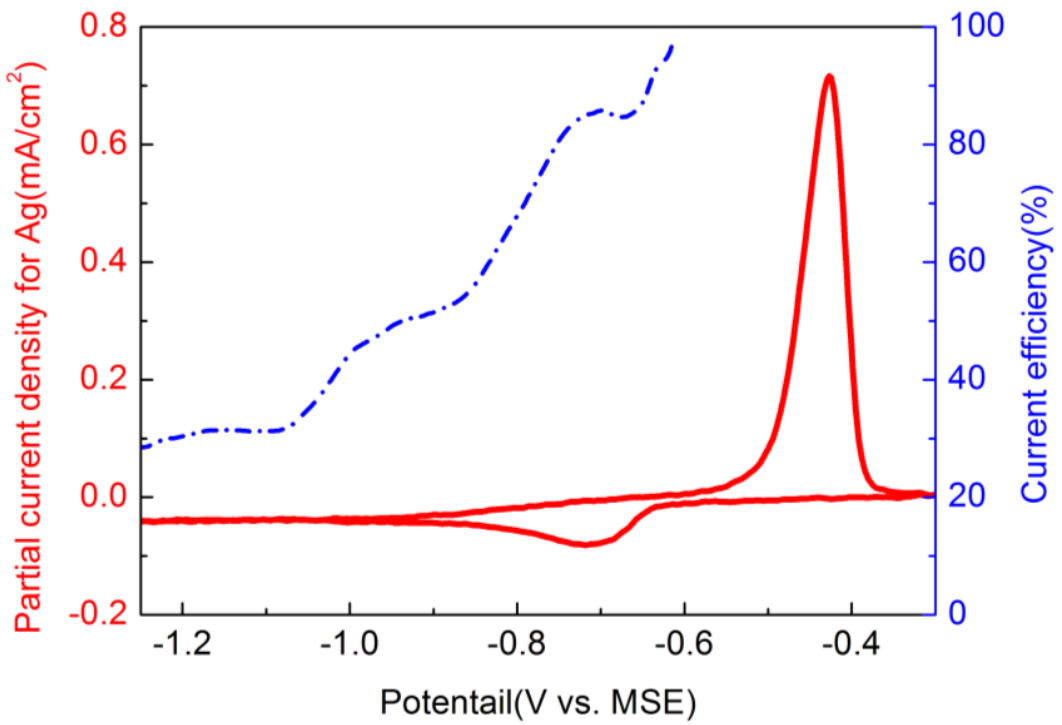

Figure 6.16 Partial current and cathodic current efficiency for Ag reduction.

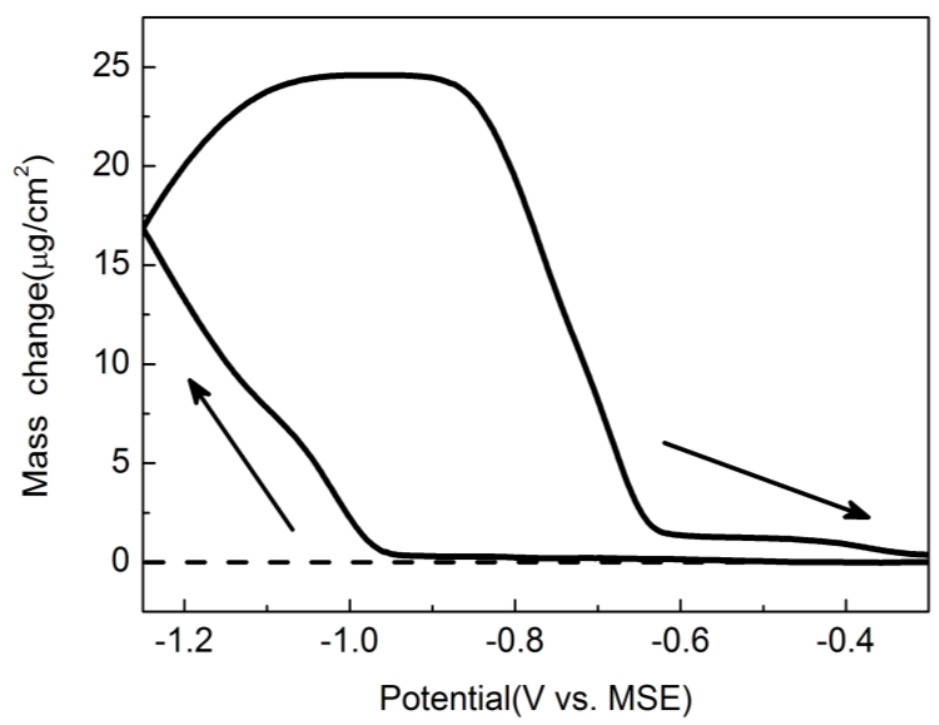

a)

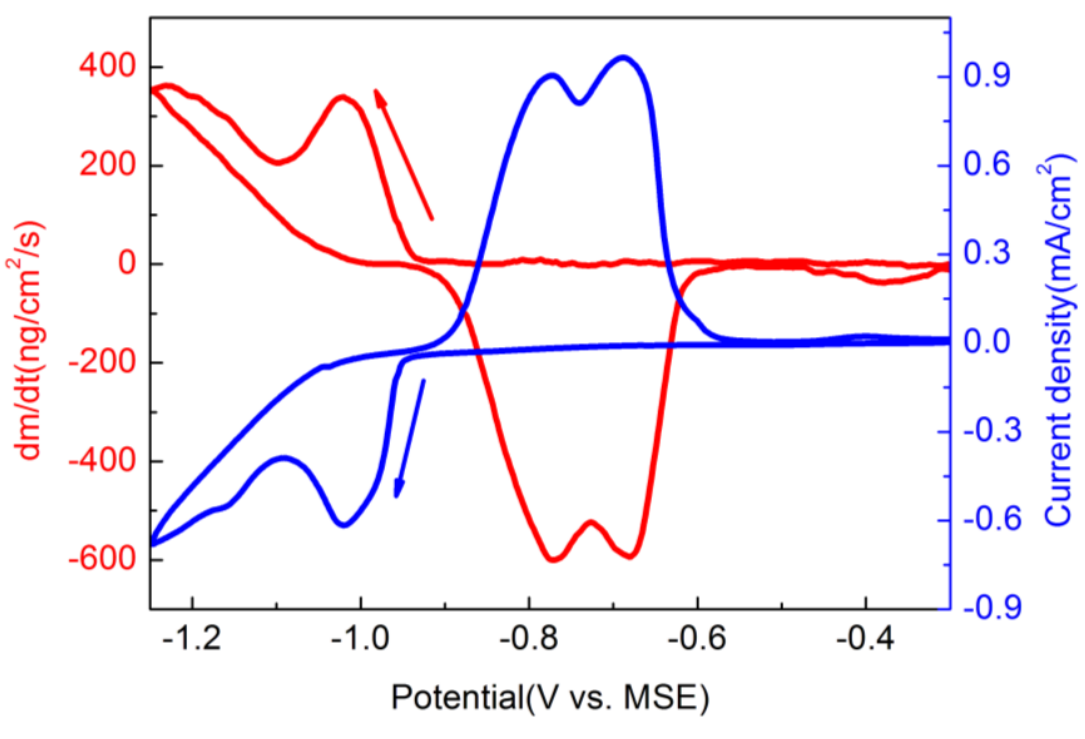

b)

Figure 6.17 a) Mass change and b) $\mathrm{CV}$ and $\mathrm{dm} / \mathrm{dt}$ data for the $\mathrm{Cu}+$ background solution. 
EQCM and $\mathrm{CVs}$ were also collected for the $\mathrm{Cu}+$ background solution. Shown in Fig.6.17 is the mass change for $\mathrm{Cu}$. The onset of $\mathrm{Cu}$ deposition occurs at $\sim-0.95 \mathrm{~V}$; $\mathrm{dm} / \mathrm{dt}$ and $\mathrm{CV}$ data shown in Fig.6.17b), demonstrate that $\mathrm{Cu}$ deposition remains relatively reversible even after complexation, as suggested by the proximity of onset of deposition and dissolution. From dm/dt data, the onset of deposition is set more precisely at $-0.935 \mathrm{~V}$, which again agrees very well with the calculation of Fig.6.14. As a result, the gap between $\mathrm{Ag}$ and $\mathrm{Cu}$ reduction is about $335 \mathrm{mV}$, as predicted by calculations.

Partial current for $\mathrm{Cu}$ deposition and cathodic efficiency are quantified using EQCM and CV data (Fig.6.18). Efficiency is above 66\% throughout the whole cathodic range.

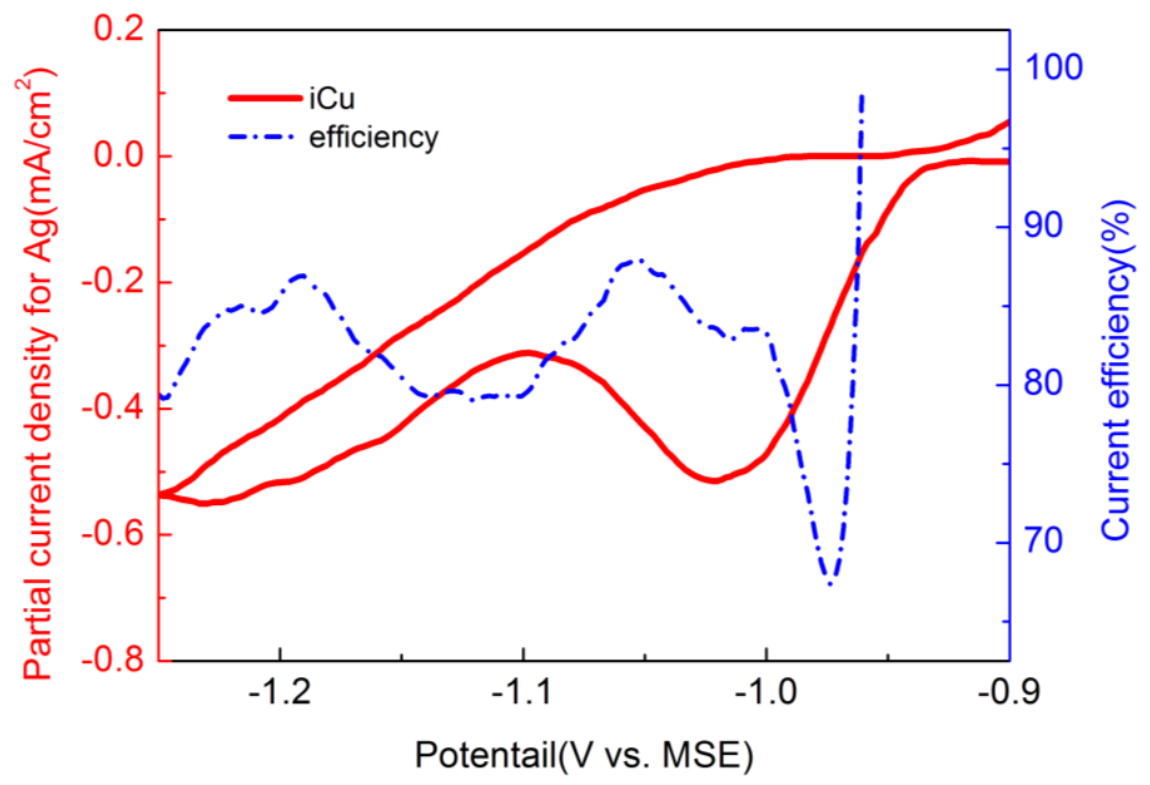

Figure 6.18 Partial current and cathodic current efficiency for $\mathrm{Cu}$.

The EQCM and $\mathrm{CV}$ data for the solution containing both $\mathrm{Ag}$ and $\mathrm{Cu}$ are shown in Fig.6.19. In the mass change data, the onset of $\mathrm{Ag}$ and $\mathrm{Cu}$ deposition are observed at potentials close to that of the corresponding single metal. 


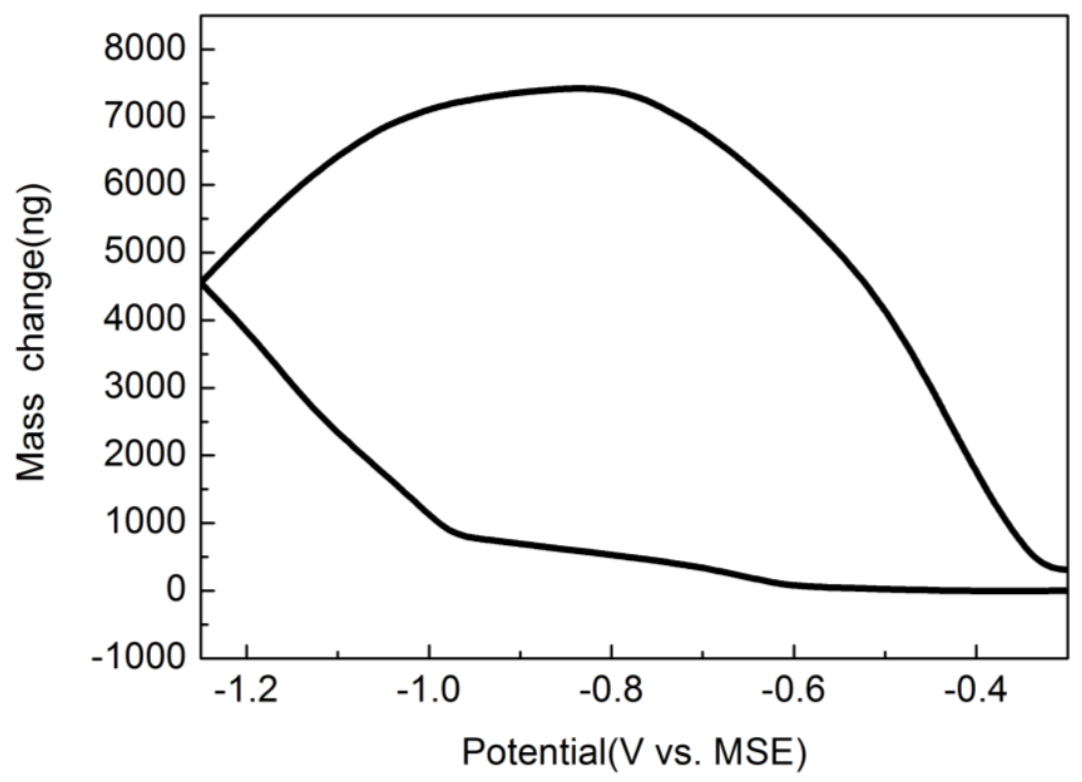

a)

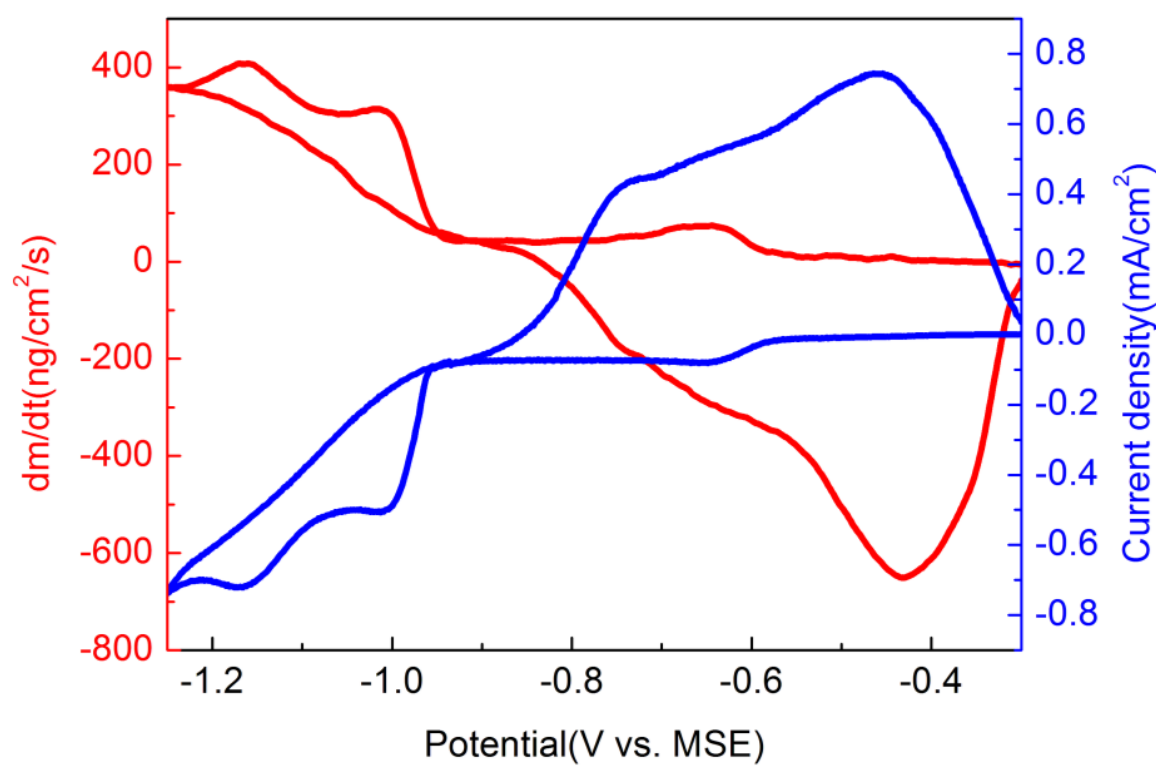

b)

Figure 6.19 a) Mass change and b) $\mathrm{CV}$ and $\mathrm{dm} / \mathrm{dt}$ data for both $\mathrm{Ag}$ and $\mathrm{Cu}$ added to the background solution.

For better comparison, the EQCM data for $\mathrm{Ag}$ only, $\mathrm{Cu}$ only and $\mathrm{Ag}+\mathrm{Cu}$ solutions are plotted together and shown in Fig.6.20. It is found that Ag deposition is depolarized by $\sim 40 \mathrm{mV}$; according to the first dissolution peak (Peak A), $\mathrm{Cu}$ deposition and dissolution processes are also depolarized by $\sim 45 \mathrm{mV}$. These observations lead us to conclude that depolarization is not due to metal interactions at the surface or in the solid state, but most probably to the fact that either $\mathrm{Ag}$ or $\mathrm{Cu}$ is complexed more strongly in the corresponding single metal solutions than in the combined solution, even though the complexing agent TU is in large excess. To 
confirm this possibility, calculation based on chemical equilibria with only TU with $\mathrm{Ag}$ and only TU with $\mathrm{Cu}$ was performed; these calculations give $\left[\mathrm{Ag}^{+}\right]=2.4 \times 10^{-13} \mathrm{M}$ and $\left[\mathrm{Cu}^{+}\right]=1.4 \times 10^{-11} \mathrm{M}$, compared with $\left[\mathrm{Ag}^{+}\right]=1.5 \times 10^{-12} \mathrm{M}$ and $\left[\mathrm{Cu}^{+}\right]=5.5 \times 10^{-11} \mathrm{M}$ with both $\mathrm{Ag}$ and $\mathrm{Cu}$ in $\mathrm{TU}$ solution, leading to a polarization of $48 \mathrm{mV}$ for $\mathrm{Ag}$ only and $36 \mathrm{mV}$ for $\mathrm{Cu}$ only, both of which are roughly in agreement with what is shown by $\mathrm{dm} / \mathrm{dt}$ data. Notice that for the $\mathrm{Cu}$ only solution, two cathodic peaks and two corresponding anodic peaks are seen; this may be related to the reduction of $\mathrm{Cu}$ from

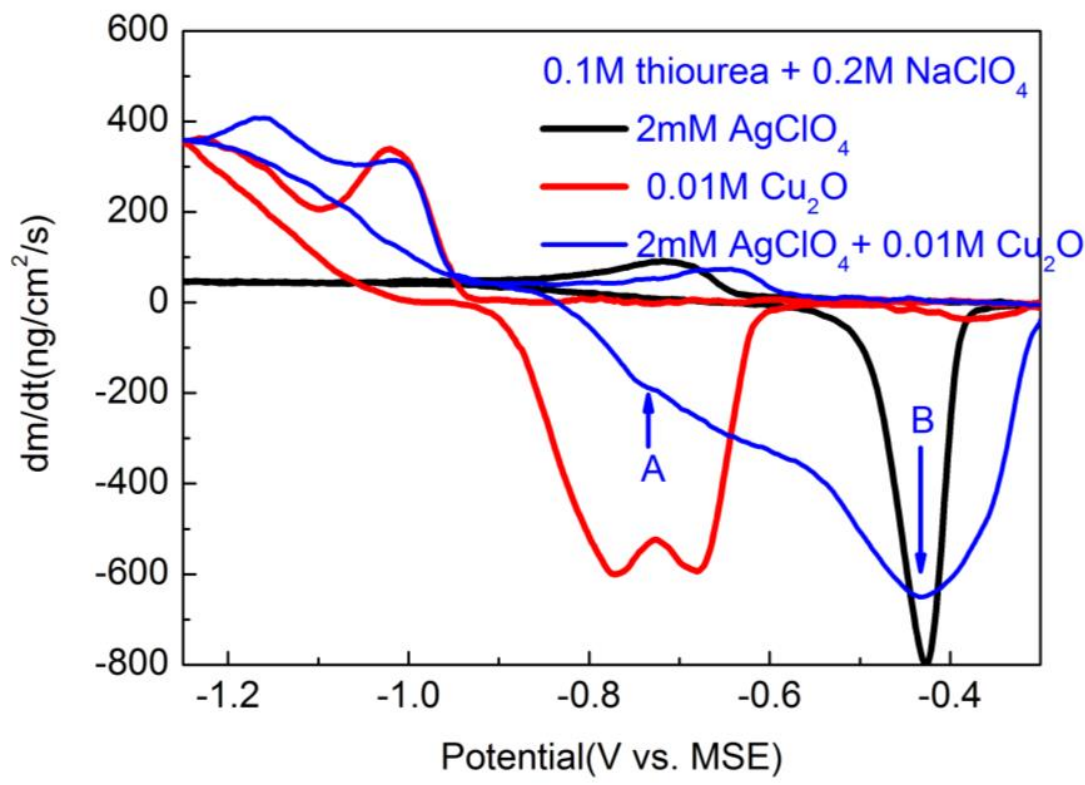

Figure 6.20 Comparison of $\mathrm{dm} / \mathrm{dt}$ data for $\mathrm{Ag}$ only, $\mathrm{Cu}$ only and $\mathrm{Ag}+\mathrm{Cu}$ solutions.

different complexes $\left(\left[\mathrm{CuL}_{2}\right]^{+}\right.$and $\left.\left[\mathrm{Cu}_{2} L_{2}\right]^{2+}\right)[19]$. Anodic peaks for the $\mathrm{Ag}-\mathrm{Cu}$ electrolyte are different from either only $\mathrm{Ag}$ or $\mathrm{Cu}$ ones. Both peaks are broader and close to each other, resulting in partially superposed $\mathrm{Cu}$ and $\mathrm{Ag}$ dissolution peaks; this is very different from what is observed from non complexed solution in Fig.6.5. This may indicate formation of phase (s) different from pure $\mathrm{Ag}$ or $\mathrm{Cu}$, e.g., solid solutions of $\mathrm{Ag}-\mathrm{Cu}$. While peaks for $\mathrm{Ag}$ and $\mathrm{Cu}$ are still observed, the microstructure of the alloys may contain pure $\mathrm{Ag}$, pure $\mathrm{Cu}$ and their solid solutions. 


\subsubsection{Composition, Morphology and Crystal structure of Ag-Cu deposits}

$\mathrm{Ag}$-Cu alloys ( $300 \mathrm{~nm}$ thick) were deposited at various potentials. The composition of these deposits was determined by EDS and it is shown in Fig.6.21. The composition is very uniform throughout the film, with less than 1at.\% spread in most cases; $\sim 2$ at.\% of $\mathrm{Cu}$ is detected already at $-0.75 \mathrm{~V}$, which is about $200 \mathrm{mV}$ positive than indicated by EQCM data of $\mathrm{Cu}$ deposition in $\mathrm{Cu}$ only solution; alloys containing 85at.\% $\mathrm{Cu}$ are grown at $-1.20 \mathrm{~V}$, with a composition plateau starting at $-1.10 \mathrm{~V}$. This is attributed to deposition occurring under diffusion limited conditions for both metal ions; the observed composition is close to the calculated ratio of 91 at.\% of $\mathrm{Cu} . \mathrm{Cu}$ atomic fraction can be calculated by eqn. (6.1):

$$
C u \%=\frac{j_{C u} / z_{C u}}{j_{C u} / z_{C u}+j_{A g} / z_{A g}} \%
$$

where $\mathrm{j}_{\mathrm{Cu}}, \mathrm{j}_{\mathrm{Ag}}$ are the partial (diffusion limited) current for $\mathrm{Cu}$ and $\mathrm{Ag}, \mathrm{z}_{\mathrm{Cu}}, \mathrm{z}_{\mathrm{Ag}}$ the electron exchanged during ion reduction (both are 1 here). $\mathrm{j}$ is approximated by equation (6.2) assuming that $\mathrm{D} \sim 10^{-5} \mathrm{~cm}^{2} / \mathrm{s}$, and a diffusion layer thickness of 0.5 $\mathrm{mm}$ :

$$
j=j_{\text {diff }}=\frac{z F D C_{0}}{\delta}
$$

where $\mathrm{F}$ is the Faraday constant, D diffusion coefficient, $\delta$ the diffusion layer thickness. The fact that $\mathrm{Cu} \%$ is slightly less than the theoretical value may be due to the slower reduction kinetics of $\mathrm{Cu}$ in comparison with $\mathrm{Ag}$. 


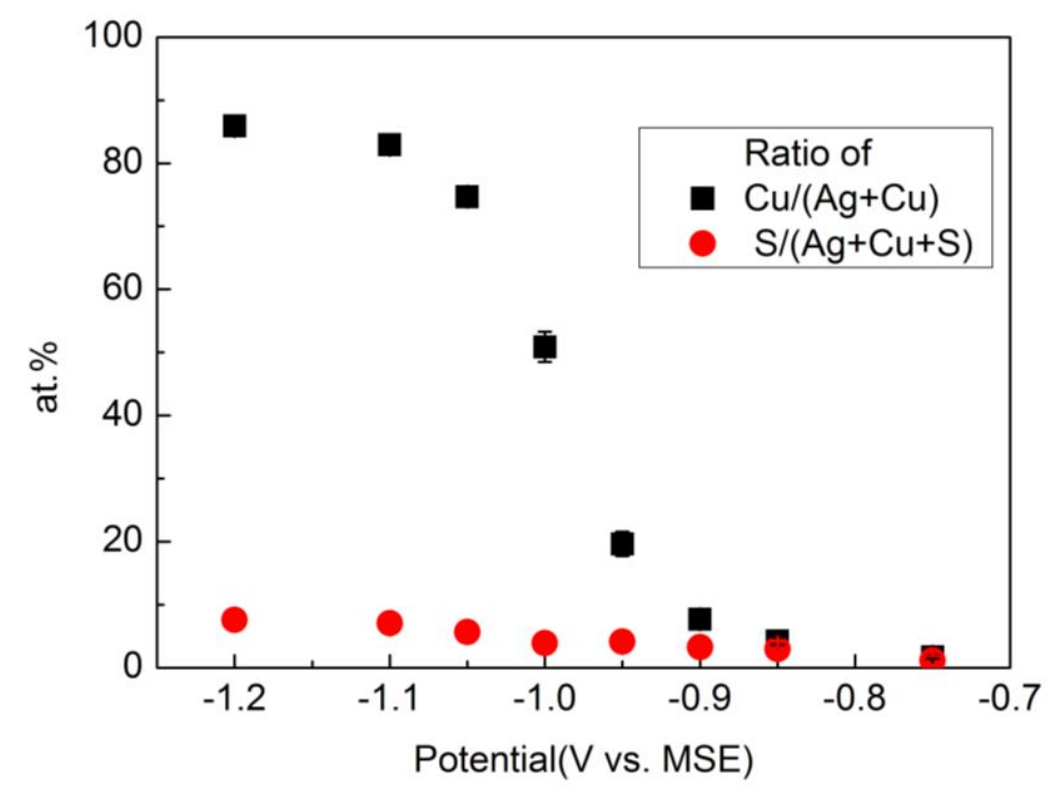

Figure 6.21 Alloy composition vs. applied potential for $\mathrm{Ag}-\mathrm{Cu}$; data were collected with at least two sets of samples and measurements at three different positions on each sample.

Besides $\mathrm{Ag}$ and $\mathrm{Cu}$, the deposits contain up to 7at.\% sulfur, $\mathrm{S}$; the fraction of incorporated S slowly increases with increasing $\mathrm{Cu}$ content, and is less than 3.5at.\% when $\mathrm{Cu}$ is less than 50at.\%; $\mathrm{S}$ reaches a plateau of 7at.\% in $\mathrm{Cu}$-rich deposits. The incorporation of sulfur into deposits grown from TU electrolytes is very common, and is mainly due to the decomposition of $\mathrm{TU}$ and the physical incorporation of $\mathrm{TU}$ fragments in the growing film [20]. We have also found a similar behavior in Ag-Ni films electrodeposited from TU electrolyte, which will be discussed in the next chapter [21].

As discussed in Section 6.2, the $\mathrm{Ag}-\mathrm{Cu}$ deposits grown from non-complexing solutions are very rough. EQCM data for TU complexing solutions also show that $\mathrm{Ag}$ reduction is under diffusion control in the potential range where alloy deposition occurs; however, deposits show very different morphologies from those deposited from non-complexing solutions. Visual inspection shows that the $\mathrm{Ag}-\mathrm{Cu}$ films are very smooth, compact and shiny, with color changing from bright gray for Ag rich films to reddish for $\mathrm{Cu}$ rich films (Fig.6.22). 


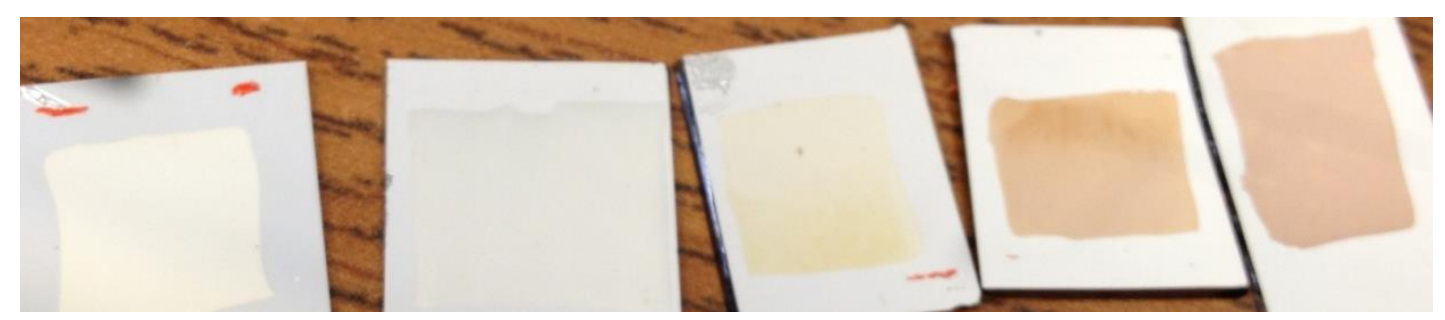

Figure 6.22 Optical images for $\mathrm{Ag}$-Cu deposits with various $\mathrm{Cu}$ contents (from left to right): 3.5 , 7.2, 19.3, 48.9 and 85 at.\%.

SEM images for these films are shown in Fig.6.23. For the Ag rich film, apparent grain sizes of $\sim 60 \mathrm{~nm}$ are observed, which are greatly reduced due to a small increase of $\mathrm{Cu}$ fraction, while grain size for pure $\mathrm{Ag}$ from $\mathrm{TU}$ solutions is about $1 \mu \mathrm{m}$ [22]. With increasing $\mathrm{Cu}$ content, the films become smoother, and grain size also decrease.
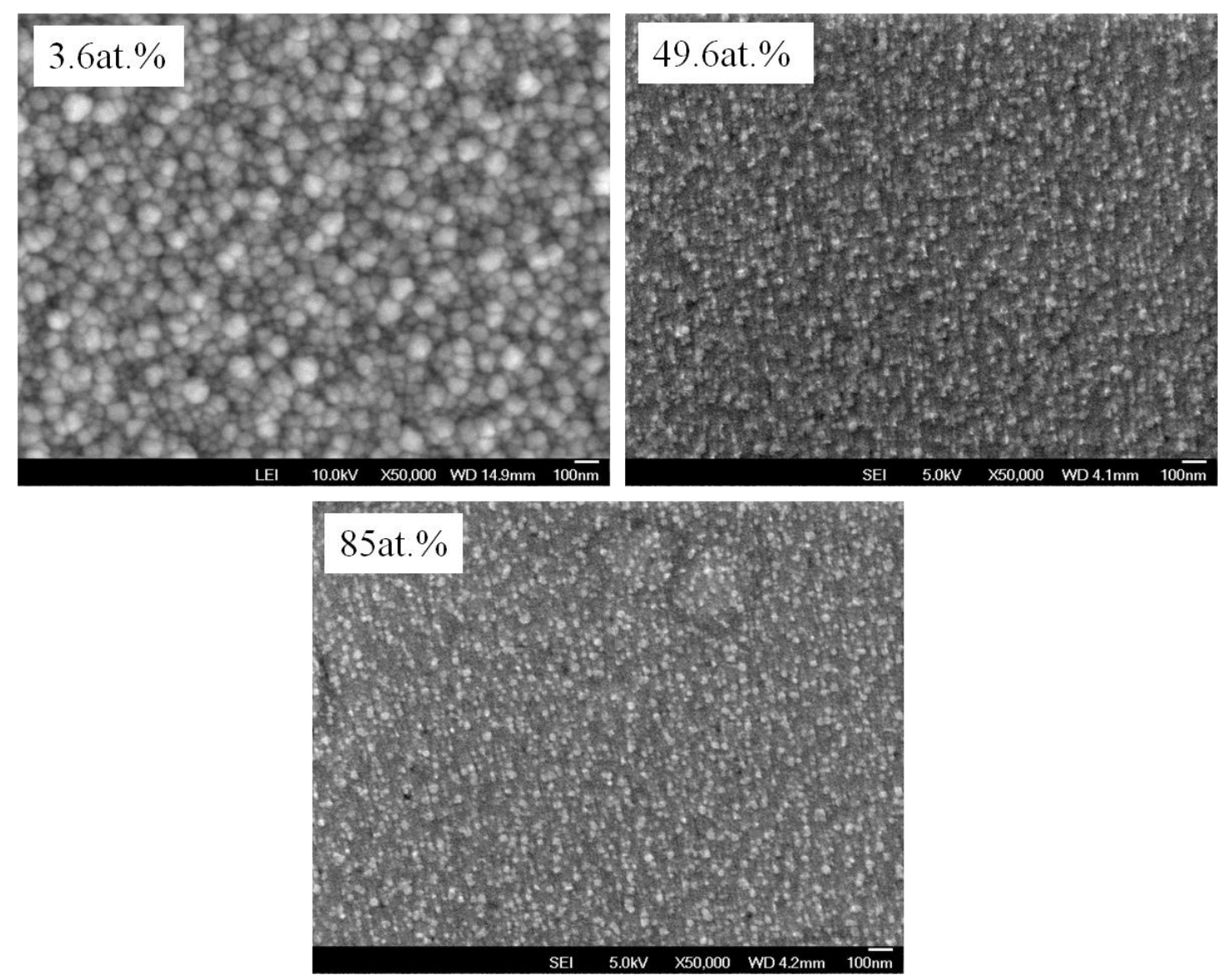

Figure 6.23 SEM images for films with various $\mathrm{Cu}$ contents.

This morphology improvement can be explained in terms of a weaker tendency for the system to undergo diffusional morphological instabilities as a consequence of the onset potentials being much closer. In the non complexing solution, $\mathrm{Cu}$ starts to be incorporated in the film when diffusion overpotential for Ag reduction (the potential 
difference between applied potential and the onset of diffusion plateau) is $\sim 300 \mathrm{mV}$ $(\mathrm{Cu}$ reduction starts at $-0.5 \mathrm{~V}$ and $\mathrm{Ag}$ diffusion limited deposition starts at $-0.2 \mathrm{~V}$ ), while in the complexing solution, about 20 at.\% of $\mathrm{Cu}(-0.95 \mathrm{~V})$ is already incorporated into the deposit at the onset of $\mathrm{Ag}$ diffusion limited deposition (-0.94 V), and $\sim 80$ at.\% of $\mathrm{Cu}$ (at $-1.10 \mathrm{~V}$ ) is found in the deposit when the diffsuion overpotential for $\mathrm{Ag}$ is only $160 \mathrm{mV}$. In other words, alloy films are deposited with a smaller overpotential from the complexing solution than the non complexing solution.

Crystal structure of the deposits is determined using XRD (Fig.6.24). A FCC (111) peak is very evident for Ag rich alloys, which gradually shifts to higher angles with increasing $\mathrm{Cu}$ content, suggesting a decrease in lattice constant, which is consistent with solid solution formation; peak width becomes larger with increasing $\mathrm{Cu}$ fraction, suggesting a decrease in grain size. The (200) peak only shows up with a very broad bump for most $\mathrm{Cu}$ rich film, when the (111) peak appears as a shoulder around the substrate peak; these films appear to have a nanocrystalline microstructure.

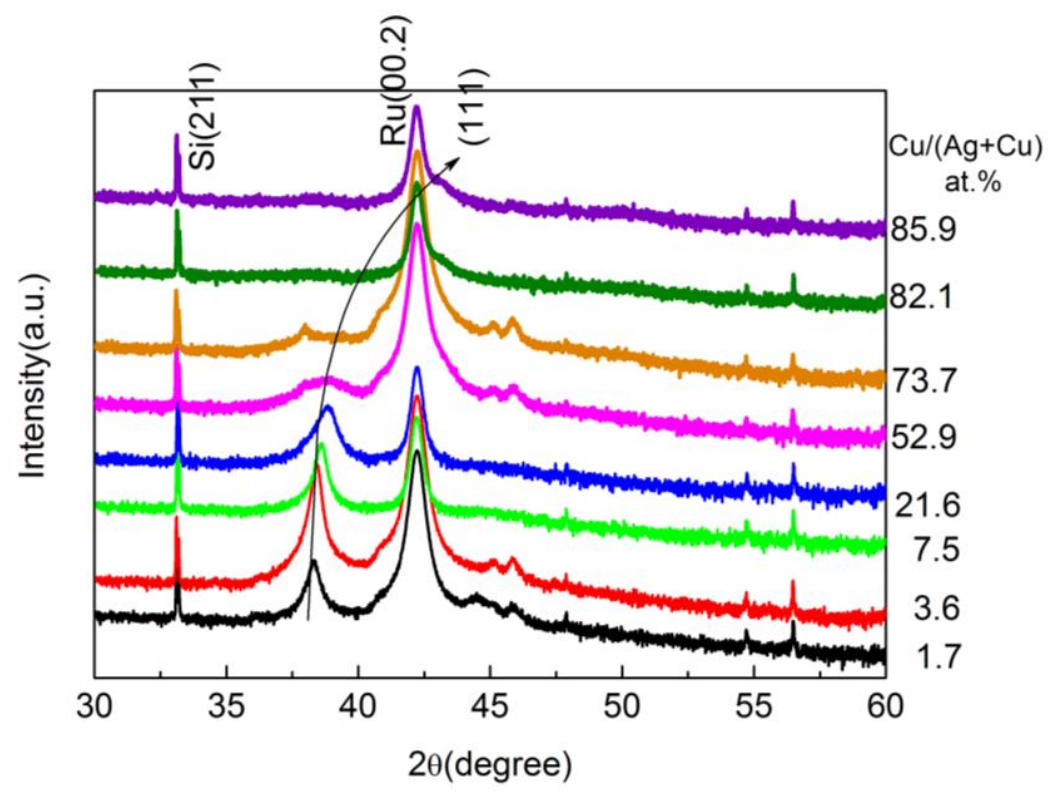

Figure 6.24 XRD patterns for $\mathrm{Ag}-\mathrm{Cu}$ deposits from complexing solution.

To assess the lattice constants and grain sizes, the (111) peak is fitted with a Lorentzian function. The lattice constants thus obtained are shown in Fig.6.25. Both theoretical calculation [7] and rapid quenched alloy data [23] show a positive 
deviation with respect to Vegard's law. The data extracted from the present work agree well with other experimental data both in the $\mathrm{Ag}$ rich and $\mathrm{Cu}$ rich ranges, but show a large deviation in the intermediate range. We hypothesize that some fraction of $\mathrm{Cu}$ in these deposits is alloyed with $\mathrm{Ag}$, forming a solid solution, while the excess fraction of $\mathrm{Cu}$ precipitates as a separate phase. From Fig.6.25, it can be estimated that $\sim 19$ at.\% of $\mathrm{Cu}$ is alloyed with $\mathrm{Ag}$ from this complexing solution, in contrast to the results reported in Section 6.2 for non complexing solutions.

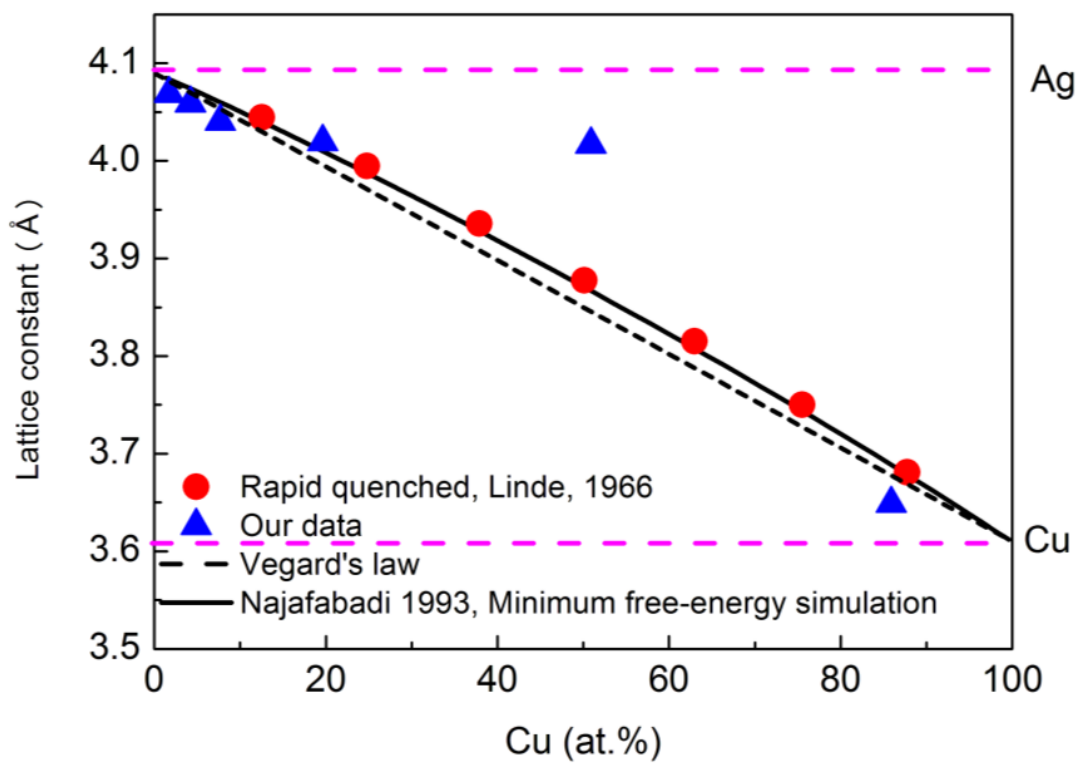

Figure 6.26 Lattice constant comparison for data from this study and literatures $[7,23]$

The grain size is evaluated using the Scherrer's equation and the results are shown in Fig.6.27. Grain size decreases as $\mathrm{Cu}$ fraction increases from $\sim 50 \mathrm{~nm}$ to only $5 \mathrm{~nm}$ at 50 at. $\%$ of $\mathrm{Cu}$. The data for $\mathrm{Cu}$ rich films cannot be extracted due to the fact that the relevant reflections are almost undetectable; it is reasonable however to conclude that the grain size for these alloys is less than $5 \mathrm{~nm}$, resulting in a nanocrystalline microstructure. 


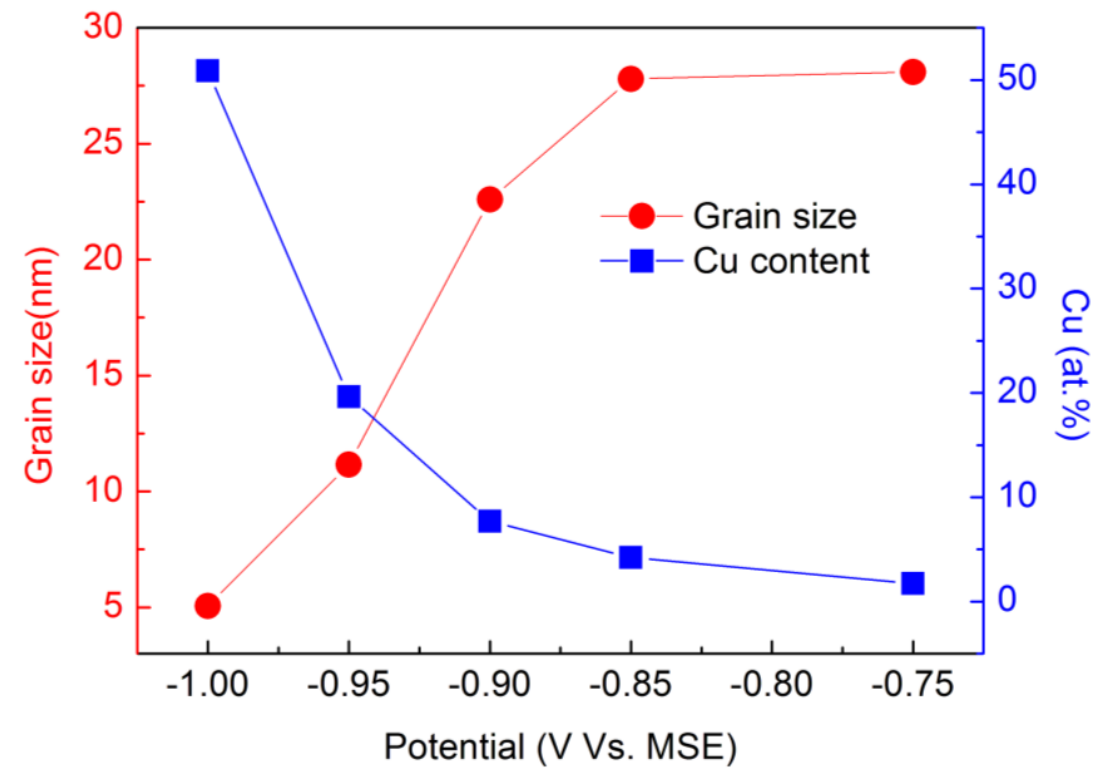

Figure 6.27 Grain size evaluated from the XRD data in Fig.6.15 as a function of potential and composition.

\subsubsection{Resistivity of Ag-Cu alloys from complexing solution}

Fig.6.28 shows the resistivity of $\mathrm{Ag}-\mathrm{Cu}$ alloys from $\mathrm{TU}$ complexing solution. Resistivity first increases and then decreases with increasing $\mathrm{Cu}$ content; all the values

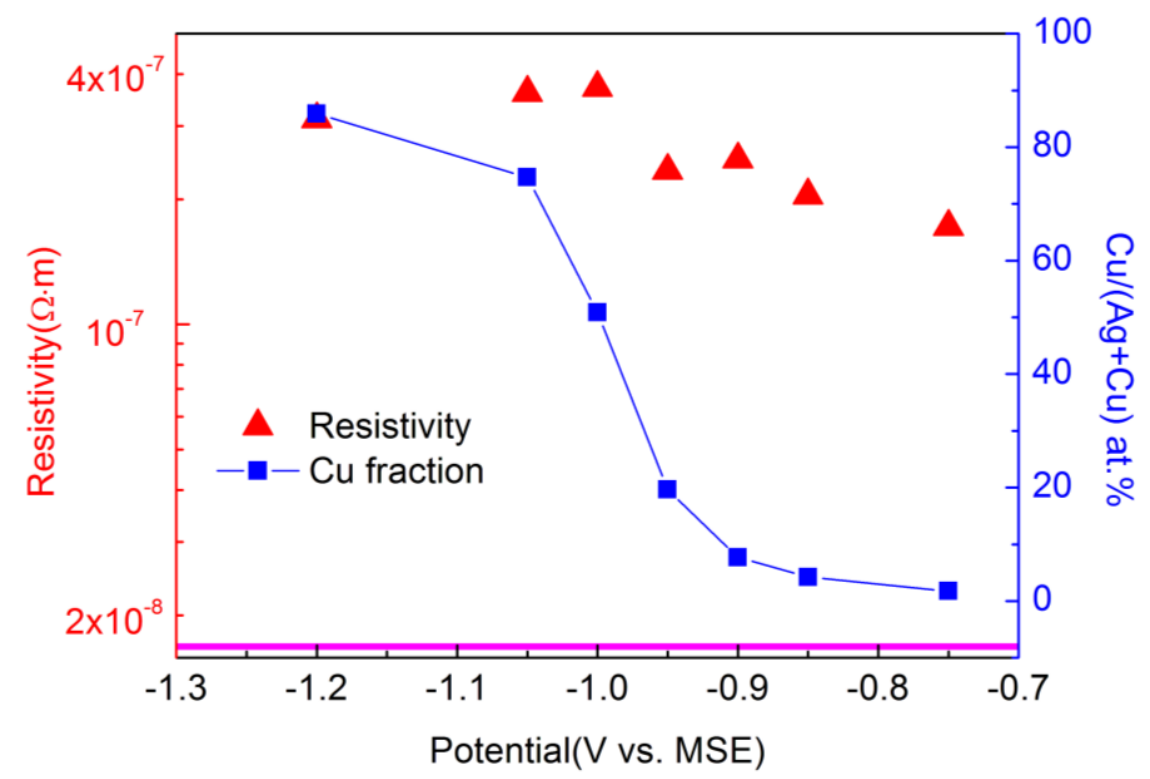

Figure 6.28 Resistivity of $\mathrm{Ag}-\mathrm{Cu}$ alloys grown from $\mathrm{TU}$ complexing solution as a function of composition; the purple line represents the resistivity for bulk $\mathrm{Ag}$ or $\mathrm{Cu}$ (notice that the resistivity for $\mathrm{Ag}$ and $\mathrm{Cu}$ is $1.59 \times 10^{-8} \Omega \cdot \mathrm{m}$ and $1.68 \times 10^{-8} \Omega \cdot \mathrm{m}$, respectively). 
are about one order of magnitude larger than those from non complexing solution, which may be due to the incorporation of sulfur impurities. The impurities serve as electron scattering centers and at the same time may decrease the grain size of the alloy, both of which lead to an increase in resistivity [24,25].

\subsection{Crystal structure of Ag-Cu alloys grown from non complexing and complexing solutions}

As discussed above, Ag-Cu alloys deposited from non complexing and complexing solutions, form phase separated precipitates and solid solutions, respectively.

In regard to the deposition from non-complexing solutions, the difference in surface mobility between $\mathrm{Ag}$ and $\mathrm{Cu}$ may be of importance in the formation of phase separated microstructures. Regardless of solutions and electrodes used, the surface diffusivity for $\mathrm{Ag}$ and $\mathrm{Cu}$ is roughly in the order of $10^{-6} \mathrm{~cm}^{2} / \mathrm{s}$ and $10^{-14} \mathrm{~cm}^{2} / \mathrm{s}$, respectively [26]. The quite mobile $\mathrm{Ag}$ adatoms may be able to move and form $\mathrm{Ag}$ clusters upon reduction, while relatively sluggish $\mathrm{Cu}$ adatoms would be practically immobile and form $\mathrm{Cu}$ clusters, leading to phase separation.

For the deposition from TU complexing solution, the diffusivity difference may not be as significant as in the non complexed solution, due to the presence of TU. Besides the complexing effect, the addition of TU in the solution may also play an important role in determining adsorption of adions onto the electrode and further surface diffusion of adatoms. TU has been found to be a good leveling agent in both Ag and $\mathrm{Cu}$ electrodeposition [27-29]; grain sizes were smaller in the presence of TU, tens of $\mathrm{nm}$ or even nanocrystalline compared with $\mu \mathrm{m}$ without TU. The absorption of TU onto the electrode, leading to the formation of metal sulfides, was found to be the source of final S segregated into grain boundaries for deposition of various metals or alloys from TU containing electrolytes [30,31]; this may inhibit the surface diffusion of metal adatoms, resulting in smaller grains and nanocrystalline structure [29]. As a 
result, both $\mathrm{Ag}$ and $\mathrm{Cu}$ adatoms may be sluggish in surface diffusion compared with that of presence of TU, which serves as hindrance, possibly in the form of sulfides. The incoming flux of reduced $\mathrm{Ag}$ and $\mathrm{Cu}$ would immobilize the adsorbed $\mathrm{Ag}$ and $\mathrm{Cu}$ adatoms, leading to mixing of $\mathrm{Ag}$ and $\mathrm{Cu}$.

However, while XRD detects and obtains average information of areas of mm [32], the presence of $\mathrm{Ag}$ or $\mathrm{Cu}$ clusters of nm scale locally entrapped cannot be ruled out in the TU complexing solution.

\subsection{Conclusion}

$\mathrm{Ag}-\mathrm{Cu}$ alloys were electrodeposited from non-complexing and $\mathrm{TU}$ complexing solutions, respectively. Rough and porous films were obtained from the non complexing solution; a very smooth surface was observed on the other hand for alloys grown from TU solution. Phase separation was observed from the former while continuous solid solution for the latter. 


\section{References}

[1] S. Strehle, S. Menzel, J.W. Bartha, K. Wetzig, Microelectron. Eng. 87 (2010) 180.

[2] M. Jun Kim, H. June Lee, S. Heon Yong, O. Joong Kwon, S.-K. Kim, J. Jeong Kim, J. Electrochem. Soc. 159 (2012) D253.

[3] M.J. Kim, S.H. Yong, H.S. Ko, T. Lim, K.J. Park, O.J. Kwon, J.J. Kim, J. Electrochem. Soc. 159 (2012) D656.

[4] X. Gao, J. Sun, M. Hu, L. Weng, F. Zhou, W. Liu, Appl. Surf. Sci. 257 (2011) 7643.

[5] S. Strehle, J.W. Bartha, K. Wetzig, Thin Solid Films 517 (2009) 3320.

[6] Y. Xie, X. Zhang, Sci. China Ser. E: Technol. Sci. 41 (1998) 348.

[7] R. Najafabadi, D.J. Srolovitz, E. Ma, M. Atzmon, J. Appl. Phys. 74 (1993) 3144.

[8] H.. Sheng, G. Wilde, E. Ma, Acta Mater. 50 (2002) 475.

[9] P. Lukinskas, I. Savickaja, V. Šukienè, A. Lukinskas, J. Coord. Chem. 61 (2008) 2528.

[10] I. V Mironov, L.D. Tsvelodub, J. Solution Chem. 25 (1996) 315.

[11] M.M. K. Hosokawa, Y. Tsuru, K. Tanimura, Proc. Interfinish (n.d.) 197.

[12] J.L. Barton, J.O. Bockris, Proc. R. Soc. London, Ser. A 268 (1962) 485.

[13] A.T. Dimitrov, S.H. Jordanov, J. Appl. Electrochem. 28 (1998) 791.

[14] B. Scharifker, G. Hills, Electrochimi. Acta 28 (1983) 879.

[15] A. Patterson, Phys. Rev. 56 (1939) 978.

[16] G.M. de Oliveira, I.A. Carlos, Electrochim. Acta 54 (2009) 2155.

[17] C.J. Doona, D.M. Stanbury, Inorg. Chem. 35 (1996) 3210.

[18] E. Herrero, L.J. Buller, H.D. Abruña, Chem. Rev. 101 (2001) 1897.

[19] M. Alodan, W. Smyrl, Electrochimi. Acta 44 (1998) 299. 
[20] S. Kuriki, M. Satō, M. Maeda, Jpn. J. Appl. Phys. 10 (1971) 604.

[21] D. Liang, Z. Liu, R.D. Hilty, G. Zangari, Electrochimi. Acta 82 (2012) 82.

[22] E. Gómez, J. García-Torres, E. Vallés, J. Electroanal. Chem. 594 (2006) 89.

[23] R.K. Linde, J. Appl. Phys. 37 (1966) 934.

[24] T. Osaka, T. Yokoshima, T. Nakanishi, IEEE Trans. Magn. 37 (2001) 1761.

[25] M. Hosseini, B. Yasaei, Ceram. Int. 24 (1998) 543.

[26] V.K. Gouda, E.N. Rizkalla, S. Abd-El-Wahab, E.M. Ibrahim, Corros. Sci. 21 (1981) 1.

[27] G.I. Lacconi, V. a. Macagno, Electrochimi. Acta 39 (1994) 2605.

[28] O. Azzaroni, P.L. Schilardi, R.C. Salvarezza, A.J. Arvia, Langmuir 15 (1999) 1508.

[29] M.S. Kang, S.-K. Kim, K. Kim, J.J. Kim, Thin Solid Films 516 (2008) 3761.

[30] T. Osaka, J. Electrochem. Soc. 146 (1999) 3295.

[31] M.S. Kang, S.-K. Kim, J.J. Kim, Jpn. J. Appl. Phys. 44 (2005) 8107.

[32] J. He, H. Sheng, J. Lin, P. Schilling, R. Tittsworth, E. Ma, Phys. Rev. Lett. 89 (2002) 125507. 


\section{Chapter 7 Electrodeposition of Ag-Ni alloys from \\ thiourea complexing solutions}

\section{Overview}

Ag-Ni alloys or bimetallic structures are of technological interest due to their remarkable catalytic [1], magnetic [2], and electrical [3] properties. In particular, Ag-Ni alloys are promising for electrical contacts and switches applications [4], where they could provide a low cost version of the widely employed hard gold alloys, currently the material of choice for commercial products [5]. More recently, nanostructured $\mathrm{Ag}-\mathrm{Ni}$ with nanoscale ferromagnetic $\mathrm{Ni}$ grains embedded in a non-magnetic Ag matrix has attracted considerable attention as a potential giant magnetoresistance (GMR) material [6]. From the thermodynamic standpoint, Ag and $\mathrm{Ni}$ are practically immiscible, both in the solid and liquid state (7); the solubility of $\mathrm{Ni}$ in $\mathrm{Ag}$ for example is at most $0.0219 \mathrm{at} . \%$ at $400^{\circ} \mathrm{C}$ [4]. Metastable, supersaturated solid solutions, both in the Ag-rich and in the Ni-rich compositional range, have been obtained in thin film form using ion beam mixing [7], laser ablation[8], or molecular beam epitaxy MBE [9], under conditions very far from equilibrium; even more notable from the structural standpoint is the report that homogeneous equiatomic nanoparticles of Ag-Ni could be obtained by gamma ray irradiation[10,11]. Such metastable configurations may exhibit unique properties, tunable via close control of the growth process. In this context, electrodeposition allows precise control of the driving force for film formation by tuning the applied potential or the current density, potentially leading to the controlled formation of metastable structures in a wide variety of alloy systems [12-14]

Electrodeposition of Ag from non-complexed electrolytes tends to occur with a high reversibility, as evidenced by the large exchange current density $\left(\mathrm{j}_{0} \sim 1.9 \times 10^{-2} \mathrm{~A} / \mathrm{cm}^{2}\right.$, $1 \mathrm{M} \mathrm{AgClO}_{4}$ at $\left.25^{\circ} \mathrm{C}\right)[15,16]$. In order to stabilize the electrolyte, decrease the 
deposition rate and inhibit dendrite formation, alkaline cyanide solutions were developed as early as 1840 and have been widely used since [17]. More recently, alternative complexing agents have been experimented with to avoid the use of cyanide and its related toxicity [16]. These solutions include for example thiosulfate [18], thiourea [19], or uracyl [16] complexes, none of which however is completely satisfactory in terms of long term solution stability [16]. Complexation of Ag should be pursued when electrodepositing Ag-Ni alloys from aqueous solutions, since the large difference in redox potentials between $\mathrm{Ag}$ and $\mathrm{Ni}\left(0.7996 \mathrm{~V}_{\mathrm{SHE}} \mathrm{vs}\right.$. $-0.25 \mathrm{~V}_{\mathrm{SHE}}$ under standard conditions) may result in Ag deposition occurring under diffusion control at the onset potential for Ni reduction. Under such conditions, morphological growth instabilities with formation of rough, porous structures are expected [20]; such morphology should be avoided, as it will impair most technological applications of these alloys. Electrodeposition of Ag-Ni from citrate complexes has been reported to result in the formation of metastable solid solutions, as deduced by $\mathrm{X}$ ray diffraction (XRD) [21]; phase separation in the films occurred upon post-deposition annealing at temperatures between 300 and $500^{\circ} \mathrm{C}$. Citrate however is not ideal to mitigate the diffusion limited deposition of $\mathrm{Ag}$ due to the relatively weak strength of Ag citrate complexes when compared with the strong complexation effect of citrate on $\mathrm{Ni}$, resulting in a large difference $(\sim 1100 \mathrm{mV})$ in redox potentials. As shown in the following, thiourea would be a better choice in this respect.

In the present study, we report on the electrodeposition of Ag-Ni alloys from thiourea based solutions. We investigate the solution chemistry by calculating relevant solution equilibria and by cyclic voltammetry experiments. We further study the crystal structure and microstructure in the alloys thus obtained, demonstrating the synthesis of metastable solid solutions. 


\subsection{Solution chemistry and electrochemical equilibria}

As mentioned earlier, the large difference in the redox potentials of Ag and Ni may lead to morphological instabilities during alloy growth; in order to avoid the formation of porous films and to facilitate solid solution formation, the redox potentials of the two metals should be shifted closer. To this end, a thiourea-based electrolyte was studied. Thiourea $\left(\mathrm{SC}\left(\mathrm{NH}_{2}\right)_{2}\right.$, TU) is extensively used in $\mathrm{Ni}$ and $\mathrm{Ni}$ alloy electroplating, where it behaves as a brightener, a leveler, and a stress-reducing agent: additional effects of $\mathrm{TU}$ include depolarization of $\mathrm{Ni}$ deposition at low $\mathrm{TU}$ concentrations (up to several $\mathrm{mmol} / \mathrm{L}$ ), and polarization of $\mathrm{Ni}$ reduction when more concentrated TU solutions are used[22,23]. Most relevant in this context however is the fact that TU complexes very strongly with $\mathrm{Ag}^{+}$, while complexation with $\mathrm{Ni}^{2+}$ is rather weak; the corresponding complexation and stability constants are reported in Table I. Various complexes may form between $\mathrm{Ag}^{+}$and $\mathrm{TU}$, as discussed in the literature $[24,25]$; in Table I we list the most widely reported complexes, and the corresponding chemical equilibria and stability constants [26,27].

Table 7.1. Equilibria and stability constants.

\begin{tabular}{|c|c|c|c|c|}
\hline $\begin{array}{c}\text { Complexing } \\
\text { agent }\end{array}$ & & Equilibrium equations & $\begin{array}{c}\text { Equilibrium } \\
\text { constants(Log) }\end{array}$ & $\begin{array}{c}\text { Equation } \\
\text { No. }\end{array}$ \\
\hline \multirow{5}{*}{$\begin{array}{c}\mathrm{SC}\left(\mathrm{NH}_{2}\right)_{2} \\
\text { (short for } \mathrm{L} \text { ) }\end{array}$} & \multirow{4}{*}{$\begin{array}{l}\text { Association } \\
\text { with } \mathrm{Ag}^{+}\end{array}$} & $A g^{+}+L \Leftrightarrow[A g L]^{+}$ & $K=7.11$ & $1^{[26]}$ \\
\hline & & $A g^{+}+2 L \Leftrightarrow\left[A g L_{2}\right]^{+}$ & $\beta_{2}=10.61$ & $2^{[26]}$ \\
\hline & & $\mathrm{Ag}^{+}+3 L \Leftrightarrow\left[\mathrm{AgL}_{3}\right]^{+}$ & $\beta_{3}=12.74$ & $3^{[26]}$ \\
\hline & & $A g^{+}+4 L \Leftrightarrow\left[A g L_{4}\right]^{+}$ & $\beta_{4}=13.57$ & $4^{[26]}$ \\
\hline & $\begin{array}{c}\text { Association } \\
\text { with } \mathrm{Ag}^{+}\end{array}$ & $\mathrm{Ni}^{2+}+L \Leftrightarrow[\mathrm{NiL}]^{2+}$ & $K=1.40$ & $5^{[27]}$ \\
\hline
\end{tabular}




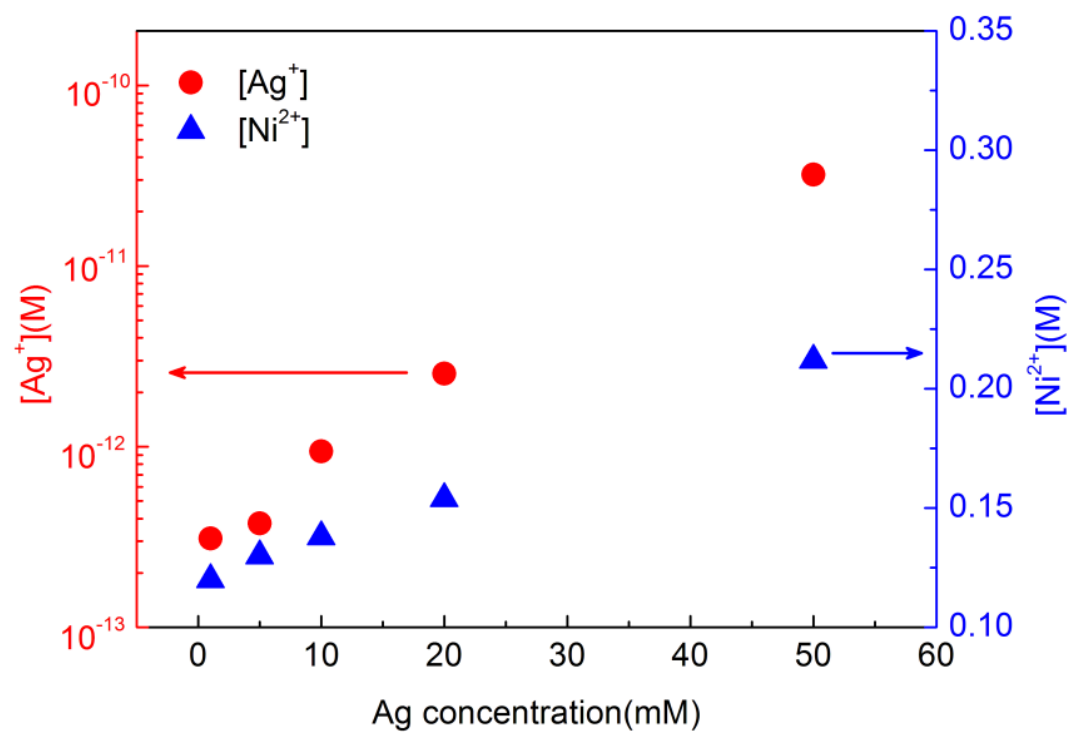

a)

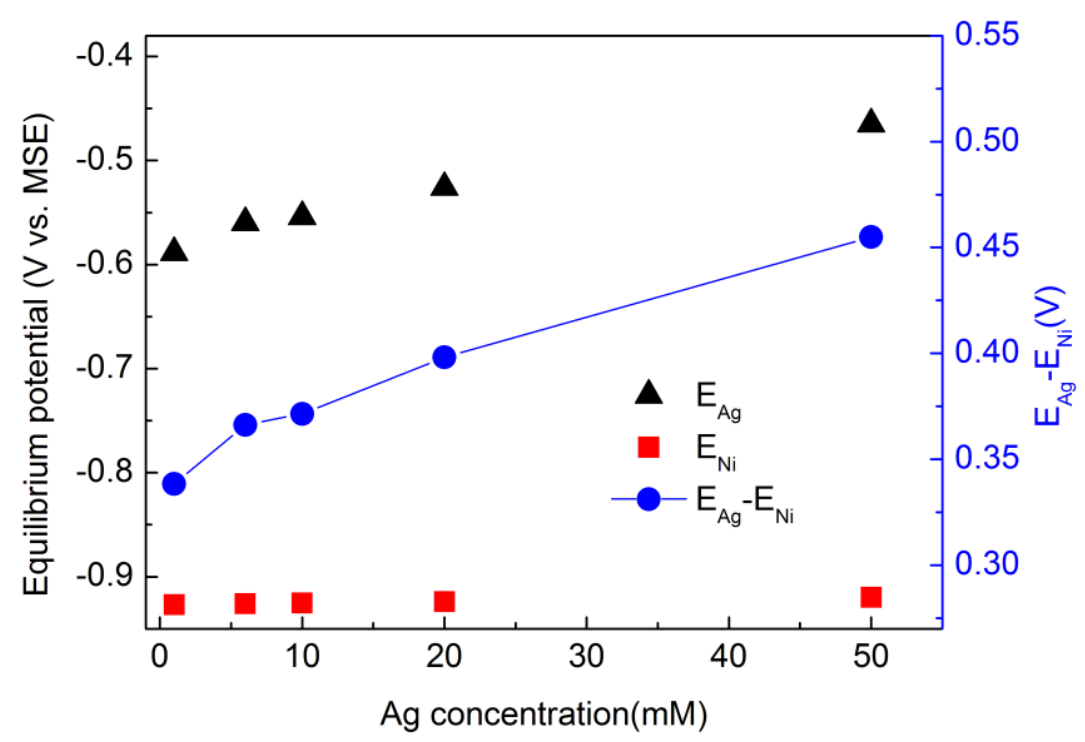

b)

Figure 7.1 a)Calculated concentration of free $\mathrm{Ag}^{+}$and $\mathrm{Ni}^{2+}$ for solution with initial $0.5 \mathrm{M} \mathrm{Ni}^{2+}$, $0.5 \mathrm{M} \mathrm{TU}$ and various TU; b) Calculated equilibrium potential for $\mathrm{Ag}^{+} / \mathrm{Ag} \mathrm{Ni}^{2+} / \mathrm{Ni}$ and their difference.

To better understand the solution chemistry, the equilibrium concentrations of free $\mathrm{Ag}^{+}$and $\mathrm{Ni}^{2+}$ were determined by simultaneously solving a series of equations for the corresponding chemical equilibria (eq. 1-5, Table I), where the initial concentrations of $\mathrm{Ag}^{+}, \mathrm{Ni}^{2+}$ and TU were set as parameters. Fig.7.1a) shows the concentration of free $\mathrm{Ag}^{+}$and $\mathrm{Ni}^{2+}$ as a function of the total $\mathrm{Ag}$ ion concentration in solution, when [TU] is 
$0.5 \mathrm{M}$ and $\left[\mathrm{Ni}^{2+}\right]$ also $0.5 \mathrm{M}$. The calculated equilibrium potentials for the $\mathrm{Ag}^{+} / \mathrm{Ag}$ and $\mathrm{Ni} / \mathrm{Ni}^{2+}$ reactions and their difference are additionally shown in Fig.7.1b). It is clearly seen that the concentration of free $\mathrm{Ag}^{+}$is greatly reduced by complexation with $\mathrm{TU}$, while the reduction in $\mathrm{Ni}^{2+}$ concentration is much more limited. As a result, the gap between the two redox potentials can be greatly decreased, down to less than $330 \mathrm{mV}$ (Fig.7.1b).

\subsection{Experimental Details}

The alloy films were deposited from aqueous electrolytes containing the metallic ions as perchlorates, using various concentrations of $\mathrm{AgClO}_{4}, \mathrm{Ni}\left(\mathrm{ClO}_{4}\right)_{2}$ and thiourea; boric acid was used as a buffer, and a supporting electrolyte was added when the total metal ion concentration was low, in order to keep the ionic strength approximately constant; a typical electrolyte formulation is reported in Table II. The solutions were prepared using ultra-pure Milli- $\mathrm{Q}^{\circledR}$ water produced in house (resistivity $18.2 \mathrm{M} \cdot \mathrm{cm}$ ); the $\mathrm{pH}$ was adjusted to 3 by adding diluted $\mathrm{NaHCO}_{3}$ or $\mathrm{HClO}_{4}$. Electrochemical and film growth experiments were conducted in a three-electrode cell using a vertical electrode configuration and an EG\&G-PAR potentiostat-galvanostat Model 263. A Pt

Table II Formulation of a typical electrolyte for Ag-Ni deposition.

$$
\begin{array}{ll}
\mathrm{AgClO}_{4} & 0.01 \mathrm{M} \\
\mathrm{Ni}_{\left(\mathrm{ClO}_{4}\right)_{2}} & 0.15 \mathrm{M} \\
\mathrm{NaClO}_{4} & 0.1 \mathrm{M} \\
\mathrm{H}_{3} \mathrm{BO}_{3} & 0.15 \mathrm{M} \\
\mathrm{TU} & 0.2 \mathrm{M} \\
\mathrm{pH} & 3.0
\end{array}
$$

mesh was used as the counter electrode, and a saturated mercury sulfate electrode (MSE) was utilized as the reference. All potential values herein are referred to MSE $\left(E_{M S E}^{0}=0.650 V_{S H E}\right)$. Neither electrolyte stirring nor deaeration were used in this work. 
Cyclic Voltammetry (CV) experiments were carried out at sputtered polycrystalline Au electrodes $(\mathrm{Si}(100) / 5 \mathrm{~nm} \mathrm{Ti} / 50 \mathrm{~nm} \mathrm{Au})$ using a scan rate of $5 \mathrm{mV} / \mathrm{s}$. The alloy films were deposited at constant potential onto two types of $\mathrm{Cu}$ substrates: $\mathrm{Cu}$ films $100 \mathrm{~nm}$ thick with a (111) preferred orientation were sputtered onto the native oxide of a $\mathrm{Si}(001)$ wafer, using a $5 \mathrm{~nm}$ Ti film as an adhesion layer; rolled $\mathrm{Cu}$ sheets (annealed,

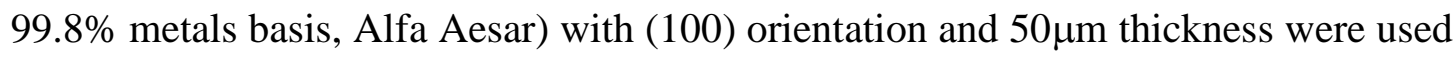
as received. Just before deposition the $\mathrm{Cu}$ substrates were dipped into $0.25 \mathrm{M} \mathrm{H}_{2} \mathrm{SO}_{4}$ for $\sim 10 \mathrm{~s}$, then rinsed with deionized water, dried with an air stream and used immediately.

Surface morphology of Ag-Ni films was examined with a JEOL JSM-6700F field emission scanning electron microscope (SEM). Film composition was measured by an energy dispersive X-ray spectroscopy (EDS) detector attached to the SEM. An accelerating voltage of $20 \mathrm{kV}$ was used; the $\mathrm{K}$ line, L line and $\mathrm{K}$ line, respectively, were used for the semi-quantitative determination of the $\mathrm{Ni}, \mathrm{Ag}$, and $\mathrm{S}$ fractions in the alloy. Crystal structure was determined by X-ray diffraction, using a Panalytical X'Pert diffractomer with $\mathrm{Cu} K \alpha$ radiation $(\lambda=1.5418 \AA)$ in the $\theta / 2 \theta$ geometry. The microstructure of as-deposited Ag-Ni films was investigated using a high resolution transmission electron microscope (HRTEM, Titan 80-300), with an acceleration voltage of $300 \mathrm{kV}$. The images were interpreted using a Fast Fourier Transform (FFT) algorithm, implemented in the Digital Micrograph (DM) software (Gatan, Warrendale, PA). The samples for TEM observation were first deposited on the $50 \mu \mathrm{m}$ thick $\mathrm{Cu}(100)$ sheet; the substrates were then thinned from the back side using ion beam milling (Gatan Model 691) to obtain a foil sufficiently thin for TEM imaging. During this process, liquid nitrogen was used to cool the sample, to avoid possible milling- and heat-induced morphological artifacts. 


\subsection{Results and Discussion}

\subsubsection{Electrochemical studies}

$\mathrm{CV}$ data are summarized in Fig.7.2. The solution containing only $\mathrm{Ag}^{+}$(thin solid line) is characterized by an onset of $\mathrm{Ag}$ deposition at $\sim 0.03 \mathrm{~V}_{\mathrm{SSE}}$, which is consistent with the redox potential of $10 \mathrm{mM} \mathrm{Ag}^{+}$calculated using the Nernst Equation. The cathodic current reaches a maximum at $-0.05 \mathrm{~V}$, followed by a plateau of about $-0.57 \mathrm{~mA} / \mathrm{cm}^{2}$ until $-0.3 \mathrm{~V}$. This value corresponds to the diffusion limited current for $\mathrm{Ag}^{+}$; assuming the diffusivity of $\mathrm{Ag}^{+}$to be $10^{-5} \mathrm{~cm}^{2} / \mathrm{s}$ and a diffusion layer thickness of $0.5 \mathrm{~mm}$, the limiting current is estimated to be $-0.19 \mathrm{~mA} / \mathrm{cm}^{2}$, in relatively good agreement with the observed value. From $-0.3 \mathrm{~V}$ to $-1.25 \mathrm{~V}$, the diffusion limited current gradually increases, which could be ascribed to the ongoing roughening of the growing $\mathrm{Ag}$ electrode; this is confirmed by visual observation of the electrode surface extracted at $-1.1 \mathrm{~V}$ after a cathodic scan, which shows a black, rough surface typical of a dendritic morphology. Hydrogen generation starts around $-1.25 \mathrm{~V}$, a value more negative than the theoretical potential, which is calculated to be $-0.83 \mathrm{~V}$ for $\mathrm{pH} 3$ solutions.

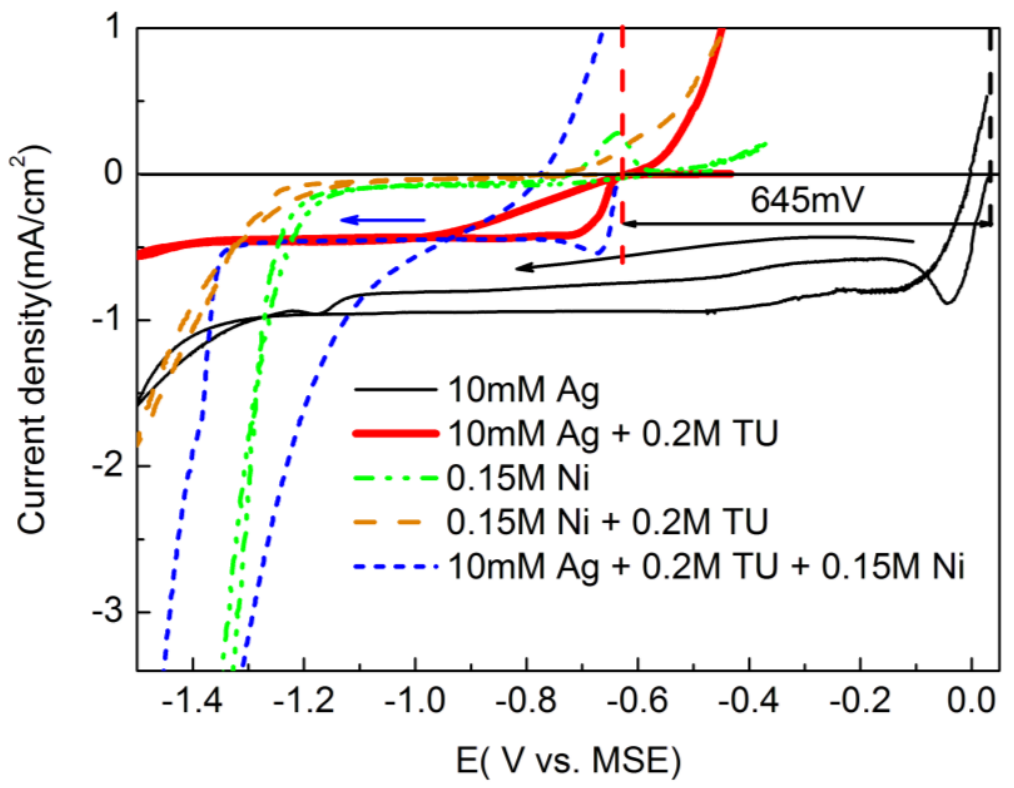

Figure 7.2 CVs for $\left.0.15 \mathrm{M} \mathrm{H}_{3} \mathrm{BO}_{3}+0.1 \mathrm{M} \mathrm{NaClO}_{4}: \mathrm{a}\right)+10 \mathrm{mM} \mathrm{AgClO}_{4}$ (black solid line); b) + $10 \mathrm{mM} \mathrm{AgClO} 4+0.2 \mathrm{M} \mathrm{TU}$ (red short dash dot line); c) $+0.15 \mathrm{M} \mathrm{Ni}\left(\mathrm{ClO}_{4}\right)_{2}$ (pink dash dot dot line); d) $+0.15 \mathrm{M} \mathrm{Ni}\left(\mathrm{ClO}_{4}\right)_{2}+0.2 \mathrm{M} \mathrm{TU}$ (orange dash dot line); e) + $10 \mathrm{mM} \mathrm{AgClO}_{4}+0.2 \mathrm{M} \mathrm{TU}+0.15 \mathrm{M}$ $\mathrm{Ni}\left(\mathrm{ClO}_{4}\right)_{2}$ (blue dash dot line). Scan rate: $5 \mathrm{mV} / \mathrm{s}$. 
The addition of $0.2 \mathrm{M}$ TU to the $\mathrm{Ag}^{+}$electrolyte (thick solid line) polarizes the onset of $\mathrm{Ag}$ deposition to $-0.645 \mathrm{~V}$, leading to a negative shift of $\sim 650 \mathrm{mV}$. Ascribing this effect to complexation of $\mathrm{Ag}^{+}$by $\mathrm{TU}$ and identifying the onset potential with the redox potential it is possible to calculate a free $\mathrm{Ag}^{+}$concentration of $10^{-11} \mathrm{M}$. Chemical equilibria calculations on the other hand estimate a concentration of free $\mathrm{Ag}^{+}$of $10^{-13}$ M, about two orders of magnitude smaller than the measured value. This discrepancy may be due to a significant nucleation overvoltage when growing Ag from complex solutions, or to the reported stability constant being measured in different media from those used here. A current plateau of $-0.46 \mathrm{~mA} / \mathrm{cm}^{2}$ was observed down to a potential of $-1.28 \mathrm{~V}$, again originated by the diffusion limited current for $\mathrm{Ag}$ reduction. In contrast with non-complexed $\mathrm{Ag}$, we observed little increase in current when scanning to more negative potentials. This suggests the formation of a smoother Ag deposit when growing from the complexing solution; such hypothesis is further validated by the bright surface obtained when the electrode is extracted after a cathodic voltage scan stopped at $-1.1 \mathrm{~V}$.

The $\mathrm{CV}$ for the $0.15 \mathrm{M} \mathrm{Ni}^{2+}$ solution (dash dot line) showed a current onset at $-1.04 \mathrm{~V}$, followed by hydrogen evolution at about $-1.2 \mathrm{~V}$. Upon addition of $0.2 \mathrm{M}$ TU (dash line), the onset of deposition is polarized to $-1.08 \mathrm{~V}$, implying a weaker complexation of $\mathrm{Ni}^{2+}$ than of $\mathrm{Ag}^{+}$by TU. As a result, the gap between the onset of $\mathrm{Ag}$ and $\mathrm{Ni}$ deposition is narrowed down from $\sim 1000 \mathrm{mV}$ to $\sim 350 \mathrm{mV}$, potentially facilitating morphological control of the film and alloy formation. Finally, the combined solution containing $\mathrm{Ag}^{+}$, $\mathrm{Ni}^{2+}$ and $\mathrm{TU}$ (short dash line) follows initially the $\mathrm{Ag}$ behavior, with a sudden increase in cathodic current at $\sim-1.35 \mathrm{~V}$, attributed to the onset of $\mathrm{Ni}$ co-deposition. Comparison with the solution without $\mathrm{Ag}^{+}$suggests a cathodic polarization of the onset potential for $\mathrm{Ni}$ by $\sim 270 \mathrm{mV}$. However, the $\mathrm{CV}$ data alone may not precisely describe the actual co-deposition process within this potential range, where hydrogen evolution may occur in parallel. Composition data vs. potential (Fig. 3) in fact show that 7 at. $\%$ of $\mathrm{Ni}$ was detected already in films deposited at $-1.06 \mathrm{~V}$, evidence that $\mathrm{Ni}$ co-deposition starts at a potential more positive than suggested by CV data. 


\subsubsection{Compositions, Morphology, crystal structure and microstructures}

In order to stabilize $\mathrm{Ag}^{+}$in solution, excess $\mathrm{TU}$ was used; $\mathrm{TU}$ is known to dissociate during Ni electrodeposition, leading to electrochemical reduction of sulfur at an applied voltage of about $-0.7 \mathrm{~V}_{\mathrm{SCE}}\left(-1.14 \mathrm{~V}_{\mathrm{SSE}}\right)$ [22]; the Ag-Ni films are thus expected to incorporate sulfur $(\mathrm{S})$ as well. In the following therefore we will identify the alloy composition by the relative $\mathrm{Ni}$ fraction $\left(\mathrm{x}_{\mathrm{Ni}}=\mathrm{Ni}\right.$ at.\%/(Ni at.\% $+\mathrm{Ag}$ at. $\left.\%\right)$ ) and by the absolute $\mathrm{S}$ fraction $\left(\mathrm{X}_{\mathrm{S}}=\mathrm{S}\right.$ at. $\% /(\mathrm{S}$ at. $\%+\mathrm{Ni}$ at.\% $+\mathrm{Ag}$ at.\% $\left.)\right)$.

The composition of the alloy films vs. applied potential for three solutions with different $\left[\mathrm{Ni}^{++}\right] /\left[\mathrm{Ag}^{+}\right]$ratios is shown in Fig.7.3a). All films have a nominal thickness of $600 \mathrm{~nm}$, calculated assuming $100 \%$ current efficiency. With increasing $\left[\mathrm{Ni}^{2+}\right] /\left[\mathrm{Ag}^{+}\right]$ ratio, $\mathrm{Ni}$ co-deposition starts at a more positive potential, as expected from the Nernst equation; correspondingly, a lower overpotential, with respect to $\mathrm{Ag}$, is needed to achieve the same Ni fraction in the alloy. EDS data reveal that sulfur is also incorporated in the films; $\mathrm{x}_{\mathrm{S}}$ increases with the Ni fraction, and reaches a plateau at a concentration $\leq 20$ at. \% (Fig.7.3b)). The observed trend can be rationalized in terms of $\mathrm{S}$ reduction being catalyzed by $\mathrm{Ni}$ reduction at low $\mathrm{Ni}$ deposition rates, leading to a $\mathrm{S}$ content approximately proportional to the $\mathrm{Ni}$ content; at high $\mathrm{Ni}$ deposition rates however, S incorporation would be limited by TU diffusion at the cathode, leading to the observed plateau [28]. 


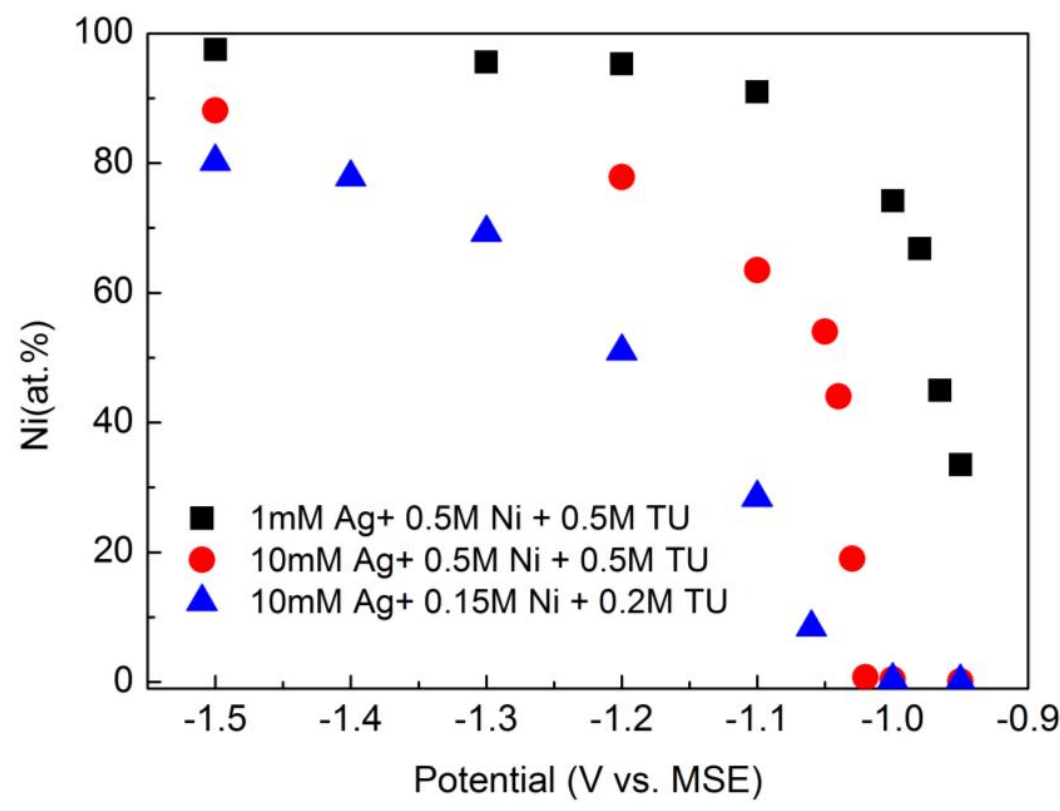

a)

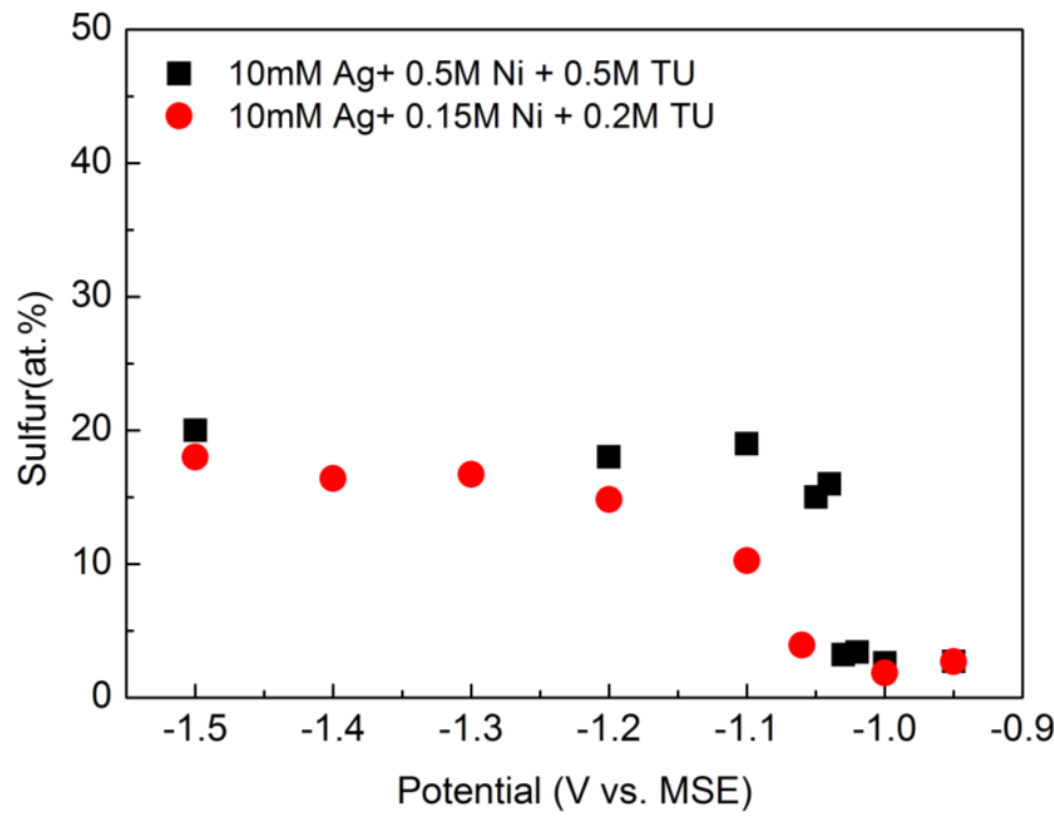

b)

Figure 7.3 Composition vs. potential for various solutions: a) ratio of $\mathrm{Ni} /(\mathrm{Ni}+\mathrm{Ag})$; b) ratio of $\mathrm{S} /(\mathrm{S}$ $+\mathrm{Ni}+\mathrm{Ag})$

The optical appearance of the films evolves with increasing Ni fraction from silvery bright and smooth to black, non-reflecting surfaces above a Ni content of about 7 at $\%$. In Fig.7.4, SEM surface micrographs of $\mathrm{Ag}-\mathrm{Ni}$ films with various $\mathrm{Ni}$ content are shown. Ag-rich films (<1 at.\% Ni) are smooth and exhibit a uniform apparent grain size of about 150nm; as Ni content increases, the surfaces become rougher, and films are less compact. The observed roughening may be due to deposition occurring at a 
larger overvoltage with increasing $\mathrm{Ni}$ content, further exacerbating growth instabilities due to the diffusion limiting conditions for Ag reduction [20].
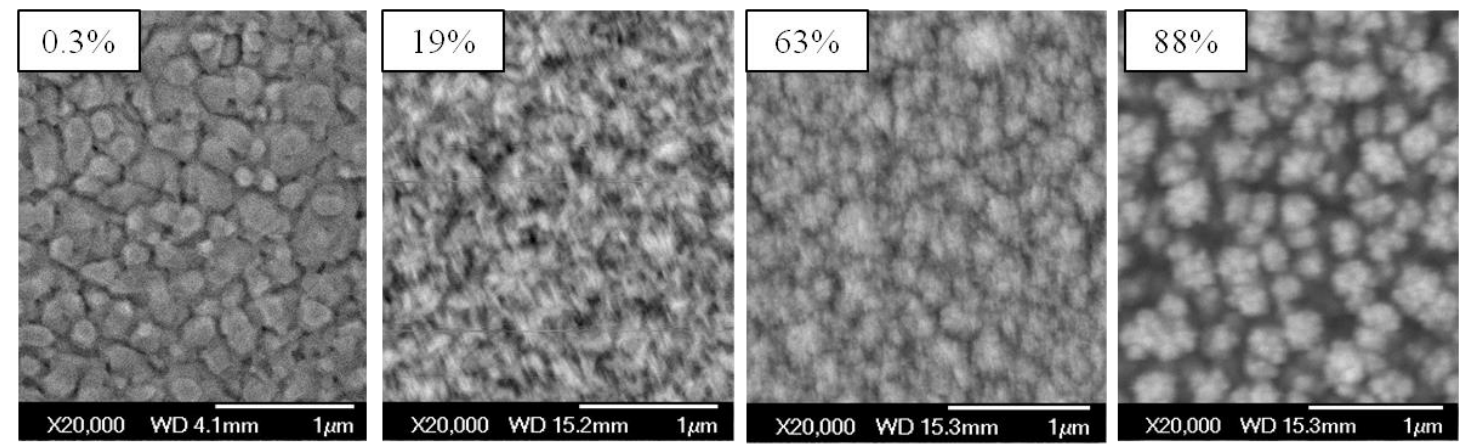

Figure 7.4 SEM image for $\mathrm{Ag}-\mathrm{Ni}$ with various $\mathrm{Ni}$ contents from the solution with $0.01 \mathrm{M} \mathrm{Ag}+$ $0.5 \mathrm{M} \mathrm{Ni}$

The fact that alloy deposition occurs under diffusion limiting conditions for $\mathrm{Ag}$ implies also a transient behavior in the initial deposition stage due to the establishment of a diffusion layer for $\mathrm{Ag}^{+}$. Compositional gradients along the film thickness due to this effect have indeed been observed in Ag-Co deposition by Gomez et al.[29] using TU as the complexing agent as well. In this work, pure Ag was initially deposited, followed by the codeposition of Ag-Co. In order to investigate possible compositional gradients in Ag-Ni films, a series of samples were plated at $-1.2 \mathrm{~V}$ with increasing deposition times from the $0.01 \mathrm{M} \mathrm{Ag}^{+}+0.5 \mathrm{M} \mathrm{Ni}^{2+}$ solution; the corresponding composition $\left(\mathrm{x}_{\mathrm{Ni}}\right)$ is shown as a function of deposition time in Fig.7.5. Films deposited for 60s show a Ni content of 62\%; in contrast, films deposited for longer times approach a constant Ni content of $\sim 76 \%$. The $\mathrm{S}$ fraction follows a similar trend. The lower Ni content in thinner deposits could be explained in terms of the time needed to develop a steady state diffusion layer as a consequence of the increased depletion of Ag ions at the interface. Assuming that the diffusion layer thickness $\delta_{N}$ increases as $\delta_{N}=\sqrt{\pi D t}$ ( $\mathrm{D}$ is the diffusion coefficient for $\mathrm{Ag}^{+}$), the time $\mathrm{t}$ needed for $\delta_{\mathrm{N}}$ to reach the thickness of $0.5 \mathrm{~mm}$ is $80 \mathrm{~s}$, in rough agreement with the time required to achieve a constant alloy composition. The observed compositional gradient will have only a limited effect on the functional properties of the alloy, since it extends only through the initial $\mathbf{7 0} \mathrm{nm}$ of the film. 


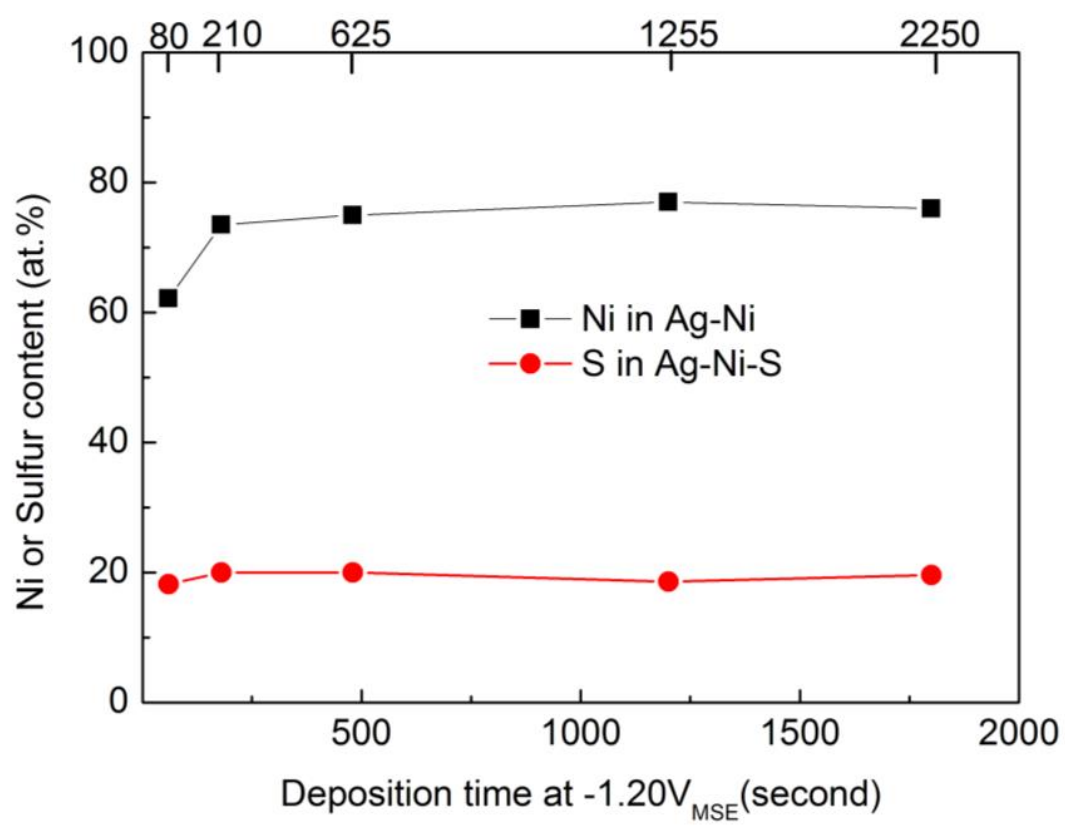

Figure 7.5 Compositions of films deposited at $-1.20 \mathrm{~V}$ with increasing deposition time from the $0.01 \mathrm{M} \mathrm{Ag}+0.5 \mathrm{M} \mathrm{Ni}$ solution; the top axis is for film thickness in $\mathrm{nm}$.

The crystal structure of alloy films with various compositions, grown from electrolytes with different $\left[\mathrm{Ni}^{2+}\right] /\left[\mathrm{Ag}^{+}\right]$ratios, were determined by $\mathrm{XRD}$; the corresponding diffraction patterns are shown in Fig.7.6. For all films, only one diffraction peak beyond that of the substrate is visible; this peak shifts to higher angles with increasing $\mathrm{Ni}$ content, and can be assigned to the (111) reflection of a face-centered cubic (FCC) structure. The gradual shift of the diffraction peak with $\mathrm{Ni}$ content suggests formation of a continuous series of solid solutions; however, the peaks are broad, and their width increases with Ni content; this could be the result of very small grain sizes, which decrease at higher Ni content, or of phase separation at very small $(\sim \mathrm{nm})$ scale [30]. XRD patterns of Ni-rich films $\left(\mathrm{x}_{\mathrm{Ni}}>90\right.$ at. $\%$ and $\mathrm{X}_{\mathrm{S}}=20$ at.\%) show practically no diffraction peak, suggesting the formation of amorphous deposits. The intensity of diffraction peaks from different elements increases roughly with their atomic number, therefore the diffraction patterns will be more sensitive to Ag-rich phases; for this reason, any hypothetical Ni-rich phase may not be detected by our instrument, especially if nanometric in size. While Ag-Ni mixtures in thermodynamic equilibrium tends to phase separate [31], the formation of metastable solid solutions has been reported in thin films grown by laser ablation [32] 


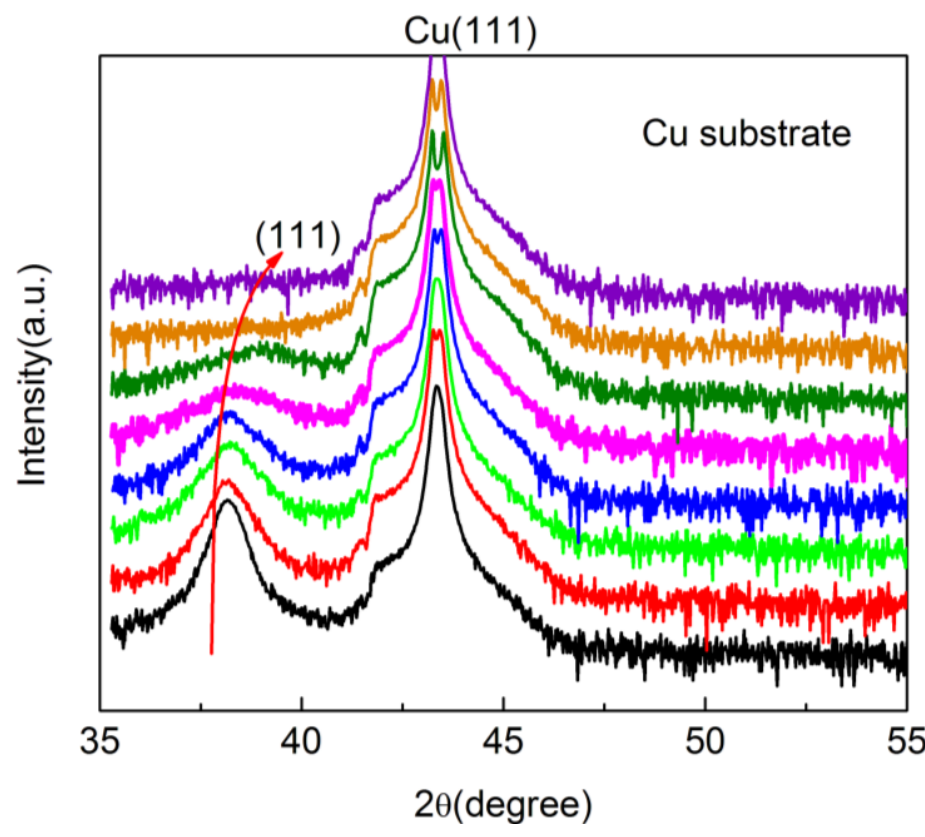

$\mathrm{Ni}$ at. $\%$

97.5

95.4

94

91

74

67

45

33.5

a)

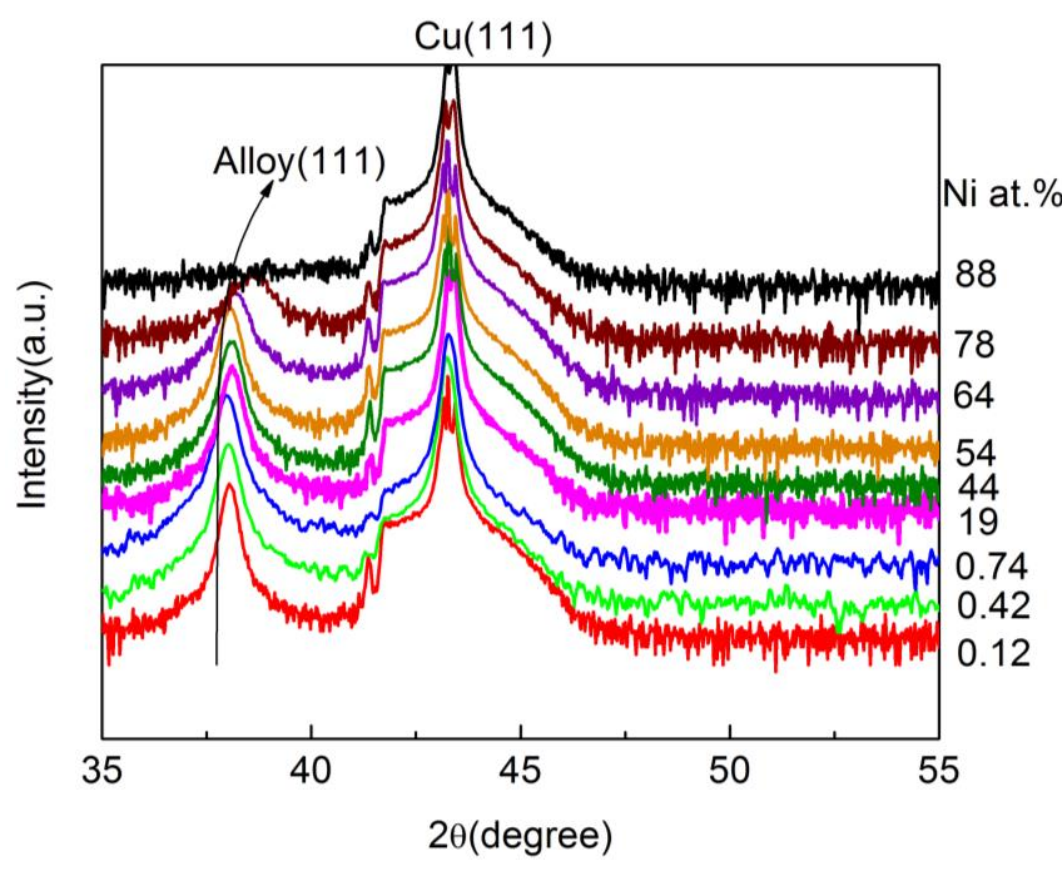

b)

Figure 7.6 XRD patterns for as-deposited films ( $600 \mathrm{~nm})$ : a) $1 \mathrm{mM} \mathrm{Ag}+0.5 \mathrm{M} \mathrm{Ni}$;

b) $0.01 \mathrm{M} \mathrm{Ag}+0.5 \mathrm{M} \mathrm{Ni}$

or electrodeposition [21]. In the attempt to estimate the extent of solid solution formation in our films, the FCC lattice constant was calculated from the (111) peak and the expected alloy composition corresponding to this lattice constant was estimated using the Vegard's law approximation. Fig.7.7a) compares the average film composition determined by EDS with the composition of the solid solution estimated with Vegard's law; the calculated solid solution compositions are much poorer in $\mathrm{Ni}$ 
than the average composition. We speculate therefore that a fraction of the Ni being deposited is alloyed with $\mathrm{Ag}$ to form $\mathrm{Ag}$-rich $\mathrm{Ag}-\mathrm{Ni}$ solid solutions, while the remaining Ni may nucleate and grow as a separate phase; such phase may not be detected by XRD due to the intrinsically lower intensity of diffraction by $\mathrm{Ni}$, or to the
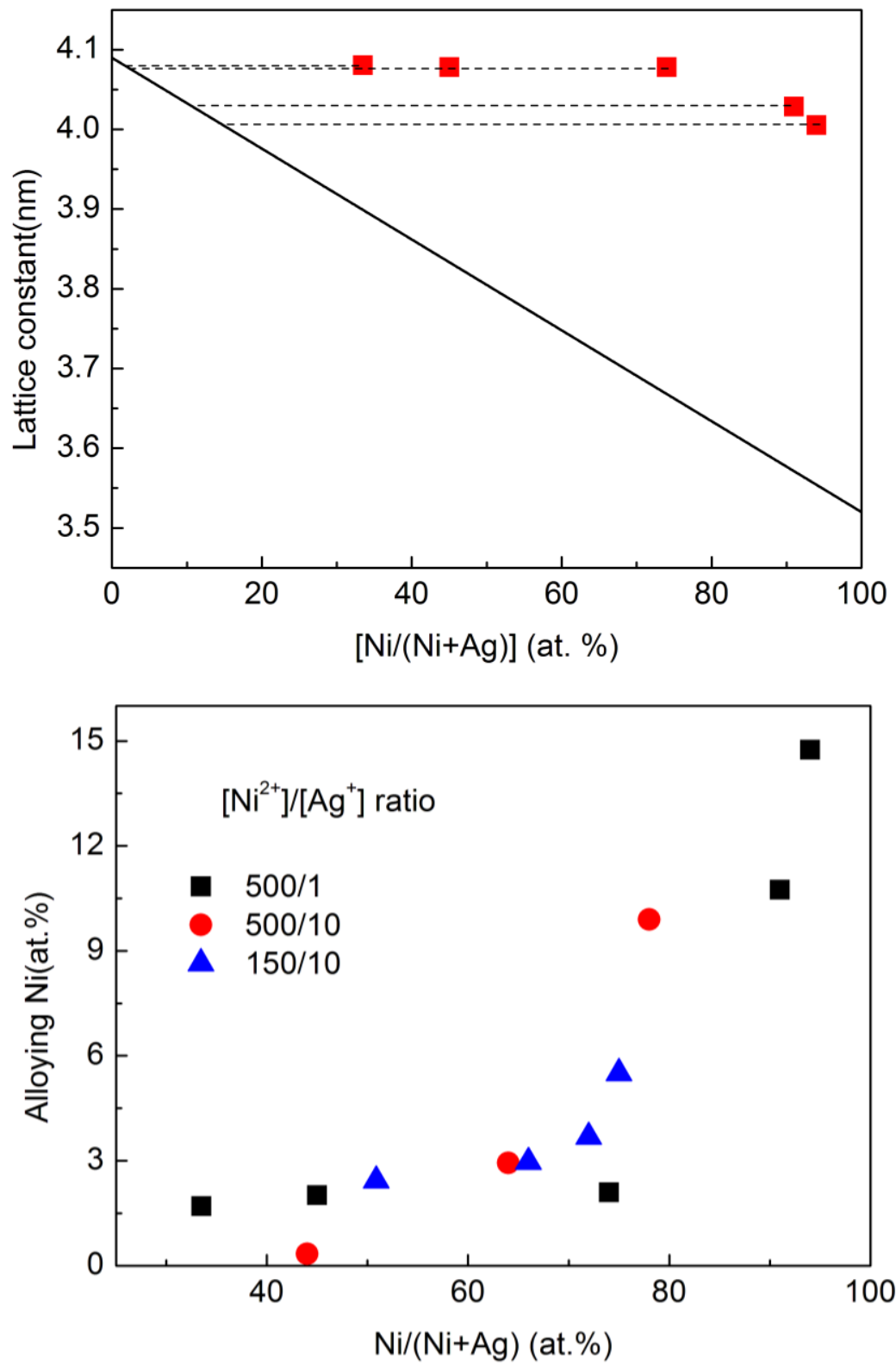

a)

b)

Figure 7.7 a) Lattice constants calculated from XRD pattern and Vegards' law for the $1 \mathrm{mM} \mathrm{Ag}+$ $0.5 \mathrm{M}$ Ni solution; b) Alloying $\mathrm{Ni}$ as a function of overall $\mathrm{Ni}$ content $\mathrm{x}_{\mathrm{Ni}}$.

$\mathrm{Ni}$ growing as an amorphous structure or as nm-scale crystallites. The grain size of this hypothetical Ni or Ni-rich phase may be further reduced as a consequence of the significant extent of S codeposition. The estimated composition of the Ag-Ni solid solution phases were further plotted as a function of the overall $\mathrm{Ni}$ content, as 
displayed in Fig.7.7b). It can be seen that only a small fraction of $\mathrm{Ni}$ is alloyed with $\mathrm{Ag}$ (at most 15 at. \%), and that such fraction increases at higher Ni content or, equivalently, at higher deposition rate. This observation seems to suggest that incorporation of $\mathrm{Ni}$ in $\mathrm{Ag}$ may be related to kinetic trapping [33] of Ni within the growing $\mathrm{Ag}$; however, comparing the fraction of $\mathrm{Ni}$ in solid solution among alloy films grown at widely different rates from electrolytes with different $\left[\mathrm{Ni}^{2+}\right] /\left[\mathrm{Ag}^{+}\right]$ ratios (Fig.7.7b), it appears that such fraction may be approximately independent of the overall deposition rate, which suggests that the extent of $\mathrm{Ag}-\mathrm{Ni}$ alloying may instead be dictated by energetic effects. This phenomenon needs further investigation.
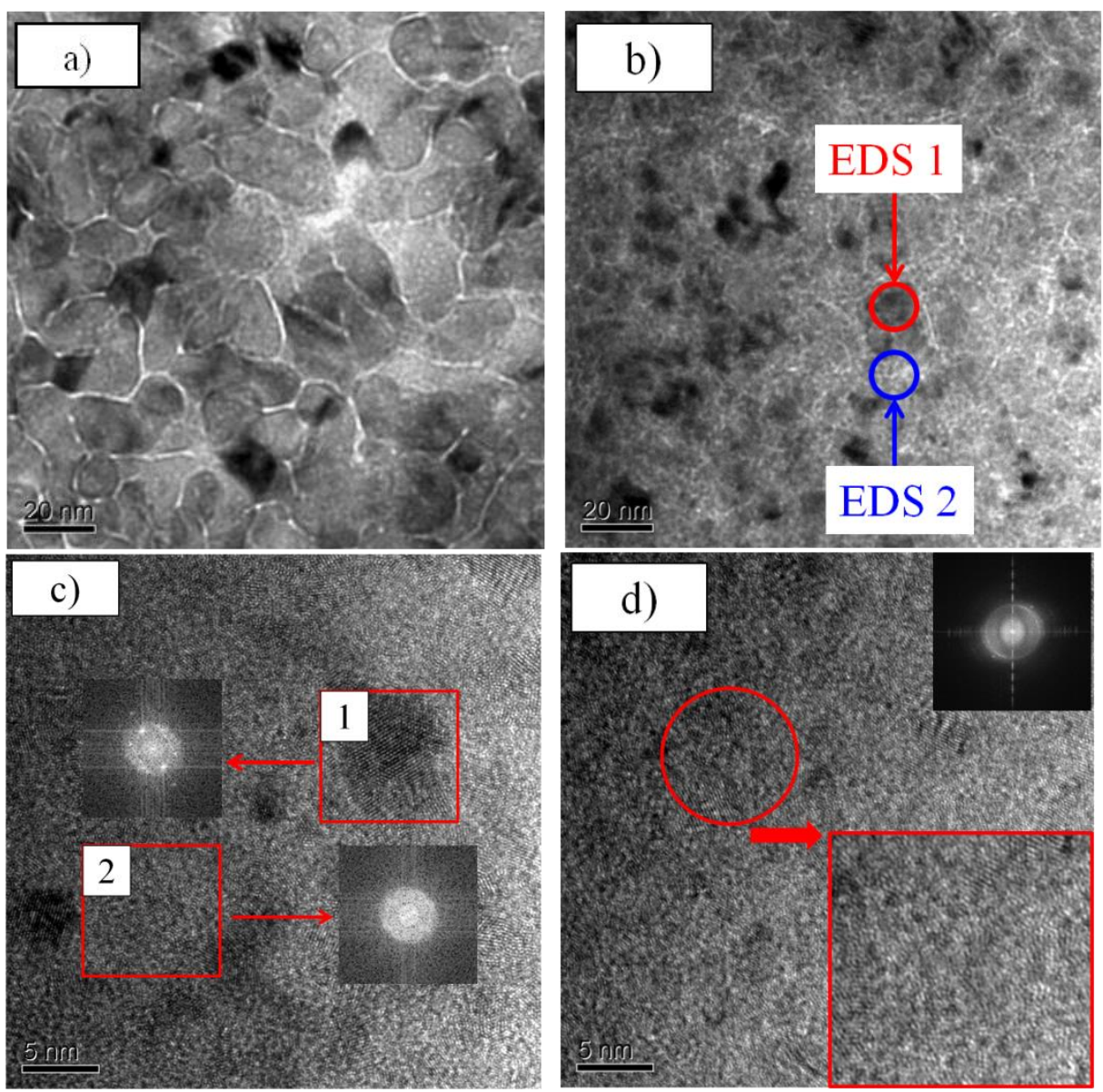

Figure 7.8 TEM micrographs and their corresponding FFTs for parts as indicated: a) <1 at. $\%$ of Ni; b) and c) 70 at. $\%$ of Ni; c) 85 at.\% of Ni. FFT for d) is by averaging the whole image.

In order to achieve a better insight into the actual microstructure of as-deposited films, High Resolution (HR) TEM was used to acquire images at atomic resolution, and FFT 
algorithms were employed to interpret such images. For the Ag-rich films (less than 1 at.\% of $\mathrm{Ni}$ by EDS), relatively uniform crystallites of $\sim 20 \mathrm{~nm}$ size are observed ( Fig.7.8 a)); HRTEM images of the same film (not shown) allow to directly extract the lattice spacing by determining the distance $a$ between atomic planes; this results in $a=0.233 \mathrm{~nm}$, very close to the result obtained with the DM software $\quad(a=0.235 \mathrm{~nm})$, and in good agreement with the theoretical spacing between successive Ag (111) atomic layers $(a=0.236 \mathrm{~nm})$. It is interesting to note that the addition of a very small amount of Ni to Ag is sufficient to strongly decrease the grain size of Ag electroplated using TU-based solutions from an apparent value of about $1 \mu \mathrm{m}$ [19] to $\sim 20 \mathrm{~nm}$. It is likely that the decrease in grain size may result in significant alloy hardening, as observed for Au-Ni or Au-Co electroplated films [34,35].

Films with $\mathrm{x}_{\mathrm{Ni}} \sim 70$ at.\% (and $\mathrm{X}_{\mathrm{S}} \sim 13 \mathrm{at} . \%$ ) (Fig.7.8 b)), exhibit smaller grains, with poorly defined grain boundaries. HR images reveal that the grain boundary regions (Fig.7.8 c), square 2) are amorphous, as confirmed by the corresponding FFT, which shows only a diffuse halo. On the contrary, clear diffraction spots are obtained for crystalline grains (Fig.7.8 c), square 1)), indicating crystallinity. Nano-probe EDS was centered on a crystalline grain (EDS 1 in Fig.7.8 b)) and at the grain boundary region (EDS 2 in Fig.7.8 b)), respectively. The measured composition within the grain was $\mathrm{x}_{\mathrm{Ni}}=65 \mathrm{at} . \%$ and $\mathrm{X}_{\mathrm{S}}=7$ at. $\%$, while at the grain boundary the result was $\mathrm{x}_{\mathrm{Ni}}=93$ at. $\%$ and $X_{S}=28$ at. \%. The EDS results may not provide the actual composition of the two regions, since perturbations from the surrounding area at such small scale are unavoidable, for example due to beam shifting or beam positioning. However, the data should be valid as a comparative compositional measurement, lending support to the hypothesis of an amorphous structure being formed at the grain boundary region, as a result of the higher Ni fraction and/or the preferential precipitation of Sulfur impurities. It should be noticed that both in the grains and at the grain boundaries, the Ni fraction is much higher than that estimated in the solid solution phases from XRD data. This suggests that the XRD reflections shown in Fig.7.6 might not be due to solid solution formation, but may be instead the consequence of compositional 
inhomogeneities resulting from the presence of adjacent, distinct nanoscale phases [30]. TEM images support this hypothesis; for example, grains of pure Ag or pure $\mathrm{Ni}$ were identified by measuring the lattice spacing between atomic layers. Figure 7.9 shows grains with lattice spacing of $0.234 \mathrm{~nm}$ and $0.202 \mathrm{~nm}$, corresponding to pure $\mathrm{Ag}$ (111) and pure $\mathrm{Ni}$ (111), respectively, confirming the multiphase nature of the films.

Films containing 85at.\% Ni (Fig.7.8 d)) exhibit a microstructure similar to that observed in the 70 at.\% Ni film, with the difference that even larger portions of the sample consist of the amorphous boundary region, while grains, about $3 \mathrm{~nm}$ in size, are widely dispersed. The amorphous region appears very similar to that reported in 60 at.\% Ni films obtained by vapor quenching [36].

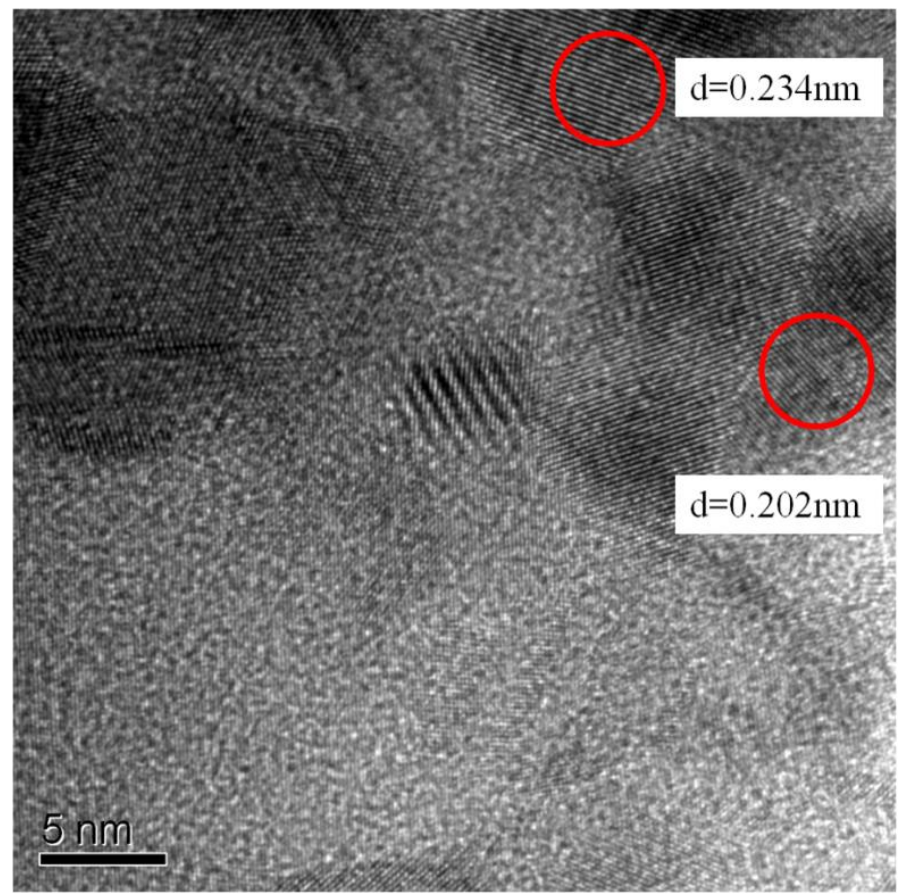

Figure 7.9 HRTEM image of a film containing overall 65at.\% Ni, showing various phases with lattice spacing corresponding to pure $\mathrm{Ag}(111)(0.234 \mathrm{~nm})$ and pure $\mathrm{Ni}(111)(0.202 \mathrm{~nm})$.

$\mathrm{Ag}$ and $\mathrm{Ni}$ are totally immiscible at room temperature; therefore the alloy phases observed by TEM are metastable. To verify this hypothesis, as-deposited films of various compositions were annealed under forming gas at $600^{\circ} \mathrm{C}$ for $30 \mathrm{~min}$. As indicated by XRD (Fig.7.10), the (111) reflections after annealing shift to the angle corresponding to $\operatorname{Ag}(111)$ irrespective of composition, and a new diffraction peak 
arises, corresponding to the $\mathrm{Ni}(111)$ or $\mathrm{Ag}$ (200) reflection, confirming that phase separation indeed occurs; furthermore, the diffraction peaks became sharper, suggesting the occurrence of grain growth processes.

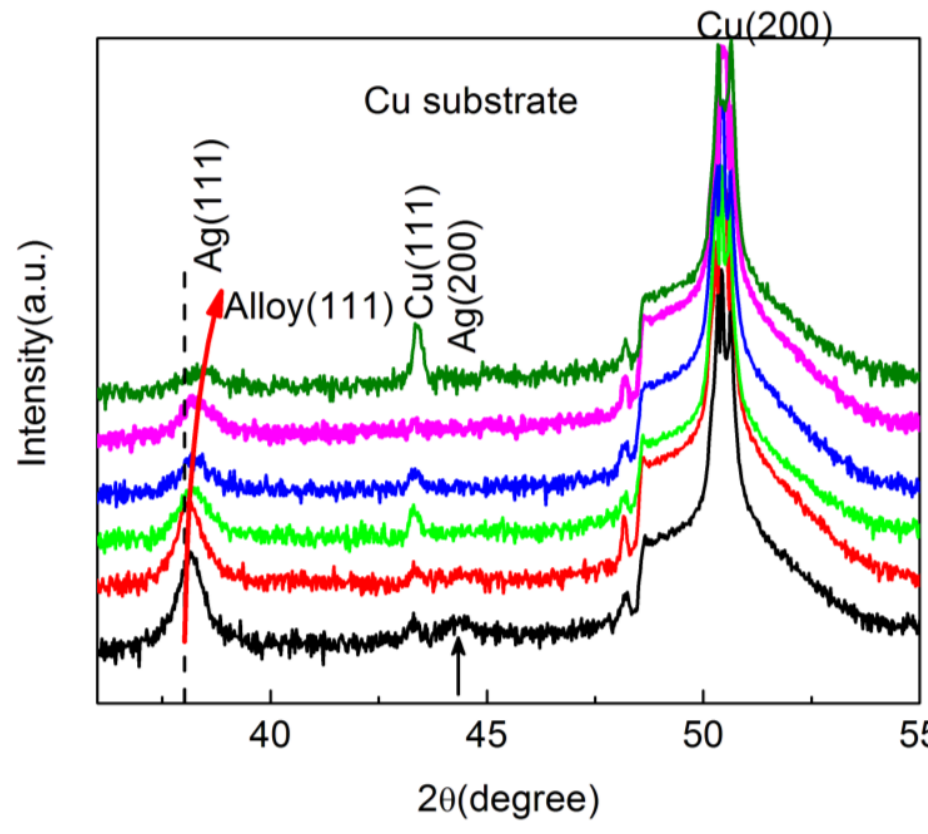

$\mathrm{Ni}$ at. $\%$

74.52

71.87

66.11

50.88

18.26

0.37

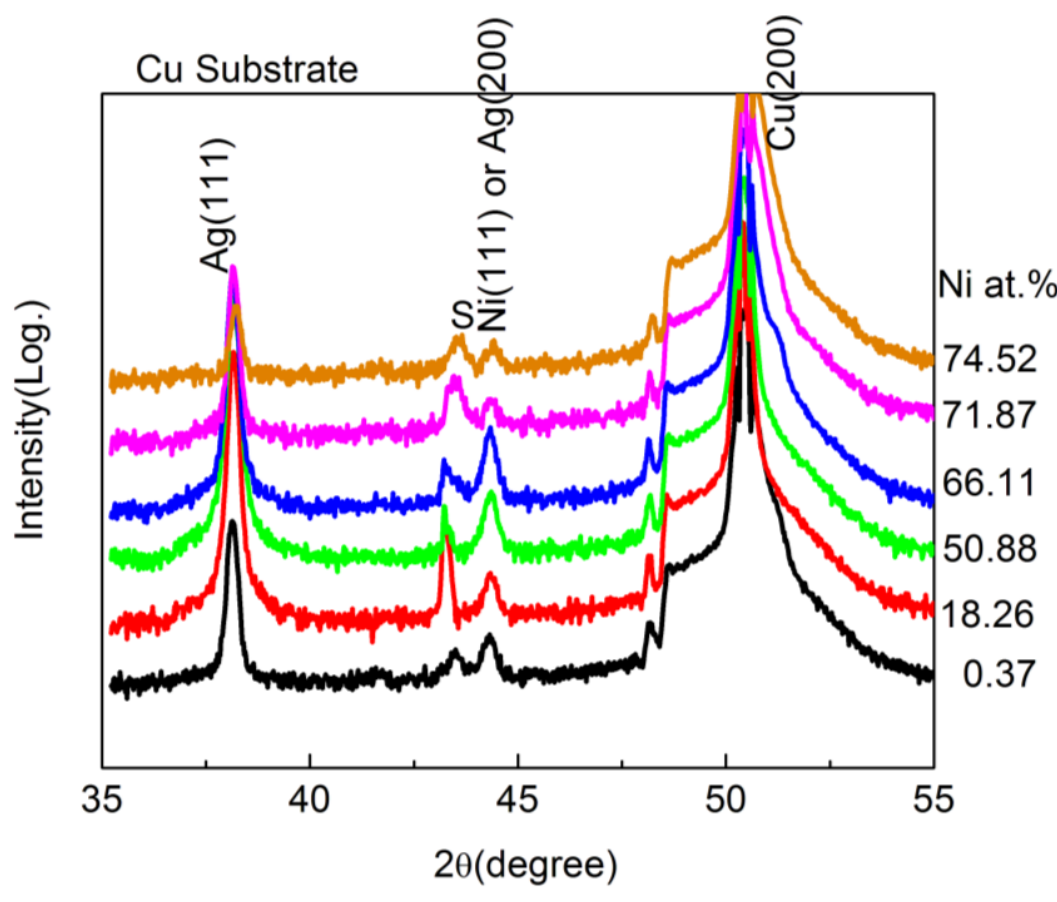

a)

b)

Figure 7.10 XRD patterns for the series of sample deposited from the $0.01 \mathrm{M} \mathrm{Ag}+0.15 \mathrm{M} \mathrm{Ni}$ : a) as-deposited; b) after annealing under forming gas for 30mins. 


\subsection{Conclusion}

Ag-Ni alloys were electrodeposited from acidic solutions, using thiourea as a complexing agent. The large difference in the onset potential for $\mathrm{Ag}$ and $\mathrm{Ni}$ deposition could be greatly reduced due to the preferential complexation of TU with Ag. XRD data suggest that as-deposited films are multiphasic, forming a Ag-rich solid solution and a $\mathrm{Ni}$ phase; after thermal annealing at $600^{\circ} \mathrm{C}$, the metastable microstructure relaxes to the equilibrium configuration consisting of the two physically separated elemental phases. TEM imaging provides a better and partly contrasting picture of the microstructure: alloy composition within crystallites is different from that deduced by $\mathrm{XRD}$; in addition, with increasing $\mathrm{Ni}$ content the average grain size progressively decreases, and larger volume fractions at grain boundaries are amorphous and richer in Ni. Up to 20at.\% of S was incorporated into the films when Ni content was more than 20 at. $\%$. 


\section{References}

[1] H. Guo, Y. Chen, X. Chen, R. Wen, G.-H. Yue, D.-L. Peng, Nanotechnol. 22 (2011) 195604.

[2] O. Proux, J. Mimault, C. Revenant-Brizard, J.R. Regnard, B. Mevel, J. Phys. Condens. Matter. 11 (1999) 147.

[3] J. Swingler, IET Sci. Meas. Technol. 5 (2011) 37.

[4] E.H. Tyler, J.R. Clinton, H.L. Luo, Solid State Commun. 13 (1973) 1409.

[5] Y. Okinaka, Gold Bull. 31 (1998) 3.

[6] L.B. Barthélémy, A., Cros, V., Duvail, J.L., Fert, A., Morel, R., Parent, F., Petroff, F., Steren, Nanostruct. Mater. 6 (1995) 217.

[7] Z.C. Li, J.B. Liu, Z.F. Li, B.X. Liu, J. Phys. Condens. Matter. 12 (2000) 9231.

[8] M. Störmer, H.-U. Krebs, J. Appl. Phys. 78 (1995) 7080.

[9] C. Azizi, A., Thompson, S.M., Ounadjela, K., Gregg, J., Vennegues, P., Dinia, A., Arabski, J., Fermon, J. Magn. Magn. Mater. 148 (1995) 313.

[10] Z. Zhang, T.M. Nenoff, J.Y. Huang, D.T. Berry, P.P. Provencio, J. Phys. Chem. C 113 (2009) 1155.

[11] Z. Zhang, T.M. Nenoff, K. Leung, S.R. Ferreira, J.Y. Huang, D.T. Berry, P.P. Provencio, R. Stumpf, J. Phys. Chem. C 114 (2010) 14309.

[12] P. Cavallotti, L. Nobili, A. Vicenzo, Electrochim. Acta 50 (2005) 4557.

[13] N. Yamachika, Y. Musha, J. Sasano, K. Senda, M. Kato, Y. Okinaka, T. Osaka, Electrochim. Acta 53 (2008) 4520.

[14] E. Rouya, G.R. Stafford, U. Bertocci, J.J. Mallett, R. Schad, M.R. Begley, R.G. Kelly, M.L. Reed, G. Zangari, J. Electrochem. Soc. 157 (2010) D396.

[15] M.M. K. Hosokawa, Y. Tsuru, K. Tanimura, M. Matsunaga, Proc. Interfinish (Jerusalem, 1984), 84, pg.197.

[16] B.-G. Xie, J.-J. Sun, Z.-B. Lin, G.-N. Chen, J. Electrochem. Soc. 156 (2009) D79. 
[17] M. Schlesinger: Modern Electroplating, 5th ed., John Wiley\& Sons, NY, USA, 2010, Chap. 5, pg.131-138.

[18] D.G. Foster, Y. Shapir, J. Jorné, J. Electrochem. Soc. 150 (2003) C375.

[19] E. Gómez, J. García-Torres, E. Vallés, J. Electroanal. Chem. 594 (2006) 89.

[20] J.L. Barton, J.O. Bockris, Proc. R. Soc. London, Ser. A 268 (1962) 485.

[21] M. Schneider, A. Krause, J. Mater. Sci. Lett. 21 (2002) 795.

[22] U.S. Mohanty, B.C. Tripathy, S.C. Das, V.N. Misra, Metall. Mater. Trans. B 36 (2005) 737.

[23] S.-K. Kim, J.E. Bonevich, D. Josell, T.P. Moffat, J. Electrochem. Soc. 154 (2007) D443.

[24] P. Lukinskas, I. Savickaja, V. Šukienè, A. Lukinskas, J. Coord. Chem. 61 (2008) 2528.

[25] D.E. Gherrou, A., Kerdjoudj H., Molinari, R., Sep. Purif. Technol. $22-23$ (2001) 571.

[26] G.M. de Oliveira, I.A. Carlos, Electrochim. Acta 54 (2009) 2155.

[27] Handbook of Preparative Inorganic Chemistry, vol. 2, 2nd Ed. G Brauer (ed) Academic Press New York, London, 1965, p. 1579.

[28] S. Kuriki, M. Satō, M. Maeda, Jpn. J. Appl. Phys. 10 (1971) 604.

[29] J. Garcia-Torres, E. Gómez, E. Vallés, J. Electroanal. Chem. 635 (2009) 63.

[30] J. He, H. Sheng, J. Lin, P. Schilling, R. Tittsworth, E. Ma, Phys. Rev. Lett. 89 (2002) 12.

[31] X.J. Liu, F. Gao, C.P. Wang, K. Ishida, J. Electron. Mater. 37 (2007) 210.

[32] R. van Ingen, R. Fastenau, E. Mittemeijer, Phys. Rev. Lett. 72 (1994) 3116.

[33] J.J. Mallett, U. Bertocci, J.E. Bonevich, T.P. Moffat, J. Electrochem. Soc. 156 (2009) D531.

[34] H. Angerer, N. Ibl, J. Appl. Electrochem. 9 (1979) 219.

[35] M.R. Lo, C. C., Augis, J. A., Pinnel, J. Appl. Phys. 50 (1979) 6887. 
[36] J. He, H. Sheng, P. Schilling, C.-L. Chien, E. Ma, Phys. Rev. Lett. 86 (2001) 2826. 


\section{Chapter 8 Conclusion and Recommendation for Future Work}

\subsection{Conclusion}

This dissertation presents a broad analysis of the process of electrochemical binary alloy electrodeposition by underpotential co-deposition (UPCD) of the less noble element. The main features of the process were clarified by use of several model systems; the main conclusions are: 1) for systems with negative enthalpy of mixing, the composition controlled by applied potential follows well the prediction by thermodynamics, even under strong complexation; 2) for systems with positive enthalpy of mixing, no general theory of alloy deposition can be drawn; either supersaturated solid solutions or phase separation obtained is atomistic phenomenon dependent.

\section{1 .1 Electrodeposition of Au-Cu alloys}

Electrodeposition of $\mathrm{Au}-\mathrm{Cu}$ alloys was carried out from weakly complexed acidic solutions, ethylendiamine (EN) complexing solutions and sulfite complexing solutions.

Deposition from weakly complexed acidic solutions follows closely the ideal UPCD behavior: the composition vs. potential follows the thermodynamically predicted behavior in the whole range of compositions, and the thermodynamic data extracted from these plots follow closely thermodynamic estimates for bulk alloys. The films obtained from this solution are rough, as they exhibit a growth instability due to the alloy growth occurring under diffusion limited deposition of Au. XRD confirms the formation of a series of continuous solid solutions.

Deposition from EN complexing solutions also follows the UPCD process; however, 
the composition vs. potential trend in $\mathrm{Cu}$-rich films deviates from the thermodynamic prediction due to $\mathrm{Cu}$ reduction occurring with a significant overpotential. Co-deposition occurs under kinetically activated deposition of Au due to the strong complexation of Au with EN, leading to a smooth morphology with small grain size $(<50 \mathrm{~nm})$. Formation of a series of continuous solid solutions is again confirmed by XRD.

Sulfite complexation is used to reverse the relative nobility of $\mathrm{Cu}$ and $\mathrm{Au}$, making $\mathrm{Cu}$ more noble than $\mathrm{Au}$. Au UPCD with $\mathrm{Cu}$ was observed by EQCM. A deviation from the thermodynamic predictions is again observed at more negative potentials. Films are smooth with fine grains.

Study on the Au-Cu system shows the possibility of tuning the driving force for electrochemical co-deposition via selective complexation, opening various opportunities for alloy synthesis.

\subsubsection{Electrodeposition of Fe-Pt alloys}

An alkaline electrolyte based on $\mathrm{Pt} \mathrm{p}$ salt and citrate/glycine complexation for $\mathrm{Fe}^{3+}$ was developed, aiming to avoid Pt deposition under diffusion limited conditions observed when using Pt chloride, resulting in the formation of dense alloys, with low oxygen content.

EQCM confirms that Fe is UPCD with Pt even under strong complexation for both metal ions. Alloy deposition proceeds under kinetic activation for Pt reduction. Films are smooth and shiny; oxygen content was significantly reduced from 30at. \% from acidic solution for equiatomic FePt to less than $6 \%$ for the whole composition range in this work. As-deposited films are FCC for Pt rich films and BCC for Fe rich films.

As-deposited near equiatomic Fe-Pt alloys are magnetically soft. Thermal annealing 
was carried out with flowing forming gas $\left(5 \% \mathrm{H}_{2}\right.$ in argon) for $1 \mathrm{~h}$ at various temperatures. $\sim 100 \mathrm{~nm}$ thick equiatomic FePt films exhibit transformation to the stable $\mathrm{L1}_{0}$ phase starting at $350^{\circ} \mathrm{C}$; coercivity of $6 \mathrm{kOe}$ was obtained after annealing at $400^{\circ} \mathrm{C}$, while annealing at $450^{\circ} \mathrm{C}$, resulting in saturation of the coercivity to $13 \mathrm{kOe}$, the highest reported for electrodeposited FePt film, at an easily reachable annealing temperature. Magnetic hardening is thickness dependent; thicker films undergo phase transformation and magnetic hardening at lower temperatures.

Bilayer and multilayer structures consisting of $15 \mathrm{~nm}$ of equiatomic $\mathrm{FePt}$ and a variable thickness of Fe-rich $\mathrm{Fe}-\mathrm{Pt}(\mathrm{Fe} 75$ at\%) were fabricated by utilizing a square wave potential technique, alternating the applied potential to values appropriate for the formation of the two alloys. It was shown that intercalation of a $5 \mathrm{~nm}$ or $10 \mathrm{~nm}$ Fe-rich layer greatly facilitates magnetic hardening compared with a single equiatomic FePt layer, showing a relative increase of coercivity of about $50 \%$ at $650^{\circ} \mathrm{C}$. The enhancement is roughly independent of the number of intercalating sublayers, suggesting that several $\mathrm{nm}$ of Fe-rich Fe-Pt coupling with FePt is sufficient to achieve a significant enhancement.

XRD patterns for Fe-Pt (Fe 75 at\%) films with $30-100 \mathrm{~nm}$ thickness show splitting of the FCC (200) peak to (200) and (002). SAED and TEM nanoprobe EDS confirmed the formation of a FCT structure with an expanded c axis, insteed of the bulk equilibrium $\mathrm{L}_{2}$ cubic structure. The possibility of forming a FCT structure is supported by published energy calculations.

\subsubsection{Electrodeposition of Au-Ni alloys}

Au-Ni alloys were deposited from weakly complexed acidic solutions. XRD indicates the formation of a series of continuous solid solutions, in contrast to the bulk phase diagram, showing the presence of a miscibility gap. Chemical shift determined by XPS confirms alloying. HRTEM reveals phase separation at the nanoscale, with the 
grain boundaries being enriched in Ni. The free energy of mixing derived by fitting the composition vs. potential behavior is similar to that of surface alloys, suggesting growth via accumulation of electrodeposited surface alloys.

\subsubsection{Electrodeposition of Ag-Cu alloys}

$\mathrm{Ag}-\mathrm{Cu}$ alloys were electrodeposited both from non-complexing, acidic electrolytes, and from TU complexing solutions. Selective complexing of Ag brings the redox potentials of the two elements closer, thus avoiding the rough and loose structures usually obtained from the non-complexing solution, and leading to smooth and shiny films. This morphological change was explained by the fact that Ag is being codeposited with $\mathrm{Cu}$ with a much smaller diffusion overpotential in the complexing solution.

Phase separation was observed by XRD for alloys deposited from the non-complexing solution, while formation of solid solutions was detected for the complexing solution. Two hypotheses have been advanced in this regard. The Potential of Zero Charge was determined by EIS in both cases, resulting in a value of $-0.60 \mathrm{~V}_{\mathrm{MSE}}$ for the former, and $-0.80 \mathrm{~V}$ for the latter. Alloy deposition occurs at potentials more positive than its PZC for the non complexed solution, at which the electrode is positively charged, repleing both $\mathrm{Ag}^{+}$and $\mathrm{Cu}^{2+}$, possibly leading to phase separation; for complexing solution, alloys were deposited at potentials more negative than PZC with electrode negatively charged, attracting both $\mathrm{Ag}^{+}$and $\mathrm{Cu}^{+}$(or their complexed species, all with positive change), probably favoring alloying.

The effect of $\mathrm{TU}$ on the phase structure formation was also considered. The adsorption of TU on the electrode, and the formation of metal sulfides may hinder the surface diffusion of $\mathrm{Ag}$ and $\mathrm{Cu}$ adatoms, leading to nanoscale $\mathrm{Ag}, \mathrm{Cu}$ or metastable alloy clusters. In this case, the observed XRD patterns would be the average signals over large areas, and may not reflect the actual nanoscale structure. 


\subsubsection{Electrodeposition of Ag-Ni alloys}

Ag-Ni alloys were electrodeposited from TU complexing solutions. Since TU complexes very strongly with $\mathrm{Ag}$ and relatively weakly with $\mathrm{Ni}$, complexation brings closer the onsets of $\mathrm{Ag}$ and $\mathrm{Ni}$ deposition. Ag rich films are smooth and gray, while $\mathrm{Ni}$ rich ones are rough and black.

XRD patterns show that the FCC (111) peak shifts to higher angles with increasing Ni content; comparison with Vegard's law shows that Ni is alloyed with Ag in Ag-rich solid solutions. HRTEM reveals the multiphasic structure of the alloys: solid solutions, pure Ni and pure Ag phase were all observed; with increasing Ni content, grain size becomes smaller, and a larger volume fraction at grain boundaries are Ni-rich and amorphous. In particular, alloys with less than 1at.\% of $\mathrm{Ni}$ are smooth and have a uniform grain size of $\sim 20 \mathrm{~nm}$, which is desirable for possible electrical contact applications, since this grain structure is helpful to obtain higher hardness and wear resistance. Annealing of the alloys at $600^{\circ} \mathrm{C}$ leads to phase separation into pure $\mathrm{Ni}$ and $\mathrm{Ag}$, and to grain growth, indicating that the as-deposited structure was metastable.

\subsection{Recommendation for Future Work}

This dissertation discusses only selected of the UPCD process. Future research in this topic could address phenomena that have not been completely clarified yet as well as new phenomena or other alloy systems. With regard to advances along the lines that have been discussed in this dissertation we suggested the following pursuits.

There is some difference for the enthalpy of mixing determined from $\mathrm{Au}-\mathrm{Cu}$ alloys deposited from various solutions, especially between the Cu UPCD with Au and Au UPCD with $\mathrm{Cu}$, with the minimum observed in Au-rich alloys for the former and for $\mathrm{Cu}$-rich alloys for the latter. This difference may be explained in terms of the actual short range order, which would require a detailed understanding of the structure of the 
deposits. High resolution TEM studies of film microstructure may help in this case.

Deposition of (100) oriented near equiatomic Fe-Pt was obtained in as-deposited films grown on $\mathrm{Cu}(100) / \mathrm{Si}$ substrates. No magnetic nor structural data were acquired due to $\mathrm{Cu}$ silicide formation after annealing, which results in a loss of substrate orientation and therefore of the film. Better substrate should be used to avoid this effect, for example, $\mathrm{Cu}$ (100), Au (100) or Pt (100) on MgO (100) may be useful to study the textured growth and the magnetic properties of oriented L10 Fe-Pt.

The high magnetic anisotropy of FePt makes it promising for bit patterned media (BPM) recording. Deposition of FePt into patterns and the magnetic performance of the same will be explored.

The different phase structures for $\mathrm{Ag}-\mathrm{Cu}$ from the two electrolytes may be studied with TEM.

Performance of Ag rich Ag-Ni alloys as electrical contacts should be assessed, for example by measuring film resistivity, mechanical hardness and wear resistance. 